Robert William Walsh

A Thesis Submitted for the Degree of PhD at the University of St Andrews

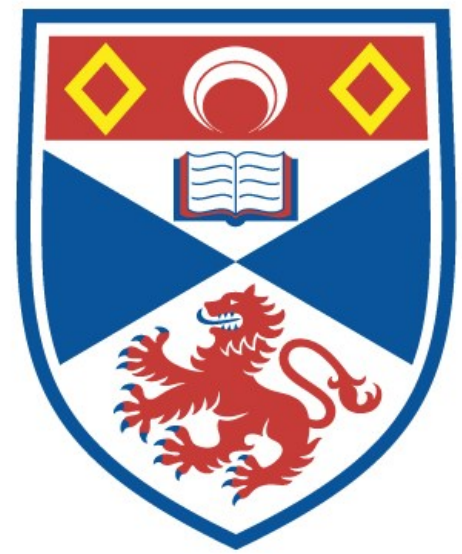

1996

Full metadata for this item is available in

St Andrews Research Repository

at:

http://research-repository.st-andrews.ac.uk/

Please use this identifier to cite or link to this item:

http://hdl.handle.net/10023/14267

This item is protected by original copyright 


\title{
Time Dependent Heating of the Solar Corona
}

\author{
Robert William Walsh
}

Thesis submitted for the Degree of Doctor of Philosophy of the University of St. Andrews

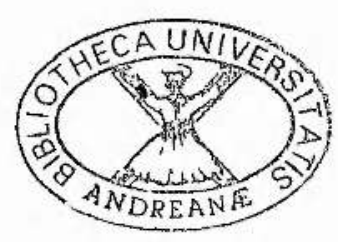


All rights reserved

INFORMATION TO ALL USERS

The quality of this reproduction is dependent upon the quality of the copy submitted.

In the unlikely event that the author did not send a complete manuscript and there are missing pages, these will be noted. Also, if material had to be removed, a note will indicate the deletion.

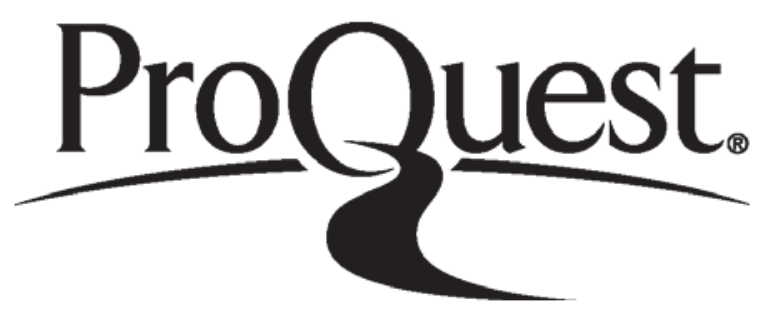

ProQuest 10171193

Published by ProQuest LLC (2017). Copyright of the Dissertation is held by the Author.

All rights reserved.

This work is protected against unauthorized copying under Title 17, United States Code Microform Edition (c) ProQuest LLC.

ProQuest LLC.

789 East Eisenhower Parkway

P.O. Box 1346

Ann Arbor, Ml 48106 - 1346 
仵 3902 


\section{Declaration}

1. I, Robert William Walsh, hereby certify that this thesis, which is approximately 50,000 words in length, has been written by me, that it is a record of work carried out by me and that it has not been submitted in any previous application for a higher degree.

date. . . . . . . . . signature of candidate. . . . . . . .

2. I was admitted as a research student in October 1992 and as a candidate for the degree of Doctor of Philosophy in October 1993; the higher study for which this is a record was carried out in the University of St. Andrews between 1992 and 1995 .

date. . . . . . . . . . signature of candidate. . . . . . . . .

3. I hereby certify that the candidate has fulfilled the conditions of the Resolution and Regulations appropriate for the degree of Doctor of Philosophy in the University of St. Andrews and that the candidate is qualified to submit this thesis in application for that degree.

date. . . . . . . . . . signature of supervisor. . . . . . . .

In submitting this thesis to the University of St. Andrews I understand that I am giving permission for it to be made available for use in accordance with the regulations of the University Library for the time being in force, subject to any copyright vested in the work not being affected thereby. I also understand that the title and abstract will be published, and that a copy of the work may be made and supplied to any bona fide library or research worker.

date. . . . . . . . . signature of candidate. . . . . . . . 


\section{Acknowledgements}

Many people need to be acknowledged for their support during my postgraduate career. I wish to thank the Department of Education for Northern Ireland for their financial support and also the School of Mathematical and Computational Sciences at the University of St. Andrews for providing the facilities for me to undertake this research. In particular, I want to mention the members of the Solar Theory Group with whom I have had fruitful and constructive discussions about my research (as well as many a bruise from five-a-side football!). Thanks should also go to Peter Jupp for his help with the statistical methods in Chapter 5. I would wish to say a huge thank-you to my supervisors, Graham Bell and Alan Hood, for their insight into and patience with my work (and also myself!).

Throughout my undergraduate and postgraduate studies I have enjoyed the warmth and fellowship of many good friends at St.Andrews Baptist Church - for this I am very grateful.

To my Mum and sister Lisa I say thank-you for their unfailing encouragement and to Heather, who at the start of my PhD was my girlfriend but now at the end of it is my wife - I couldn't have done it without you.

Contrary to what is contained in this thesis, below reveals how I believe the Sun actually works!! (Copyright G. Larson)

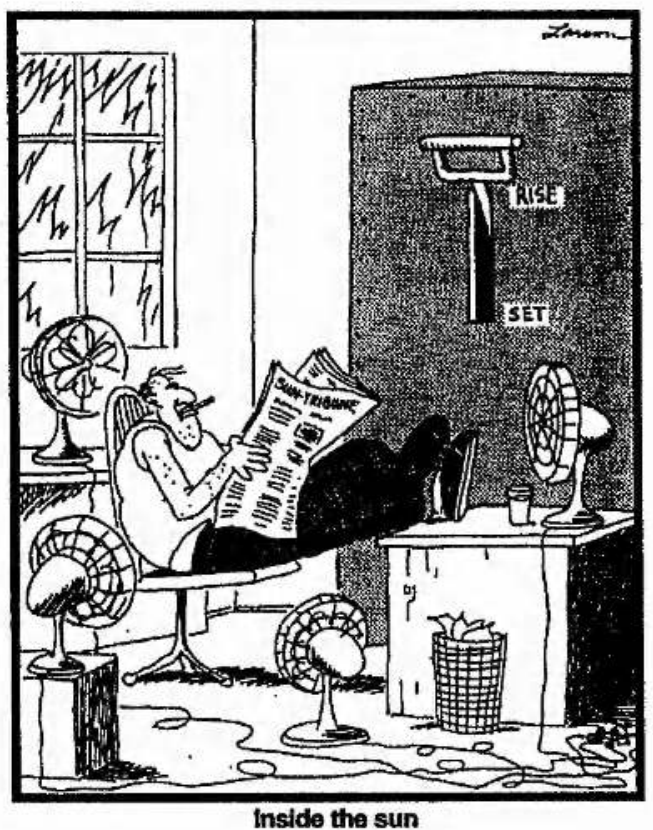

Inside the sun 
To Him who is able to do immeasurably more than all we ask or imagine.

(Ephesians 3:20) 


\begin{abstract}
The problem of how the Sun's corona is heated is of central importance in Solar Physics research. In this thesis, a model is constructed of a typical coronal magnetic loop in order to investigate the response of coronal plasma to a time-dependent heating source. It is not the aim of the research to study in detail a particular heating mechanism but rather to understand the important features arising from time-dependent heating in general. A time-varying energy input into the coronal loop is required because it is likely that none of the suggested theoretical heating methods can provide a constant supply of heat to the corona.

The magnetic field is taken to be strong enough that the loop dynamics reduce to a one-dimensional problem along the field. In addition, it is assumed that the radiative timescale in the corona is much longer than the sound travel time and thus, the plasma evolves isobarically. The thermal equilibria profiles along the coronal loop are then investigated for a simplified form of the optically thin radiation.

Initially, a heating function that displays a regular, sinusoidal variation in time is introduced and it is found that there is a critical heating frequency above which a hot coronal loop solution can be maintained and below which the plasma temperature cools to chromospheric values. Pulse heating and the deposition of random-sized energy quanta in a loop are also investigated.

An evaluation of the isobaric assumption to the corona is presented by allowing sound waves to propagate back and forth along the loop. It is found that the system can exhibit isobaric-like behaviour provided the acoustic timescale is short enough.

Possible extensions of the developed loop model are discussed as well as the implications of time-dependent heating upon observations from the SOHO satellite.
\end{abstract}




\section{Contents}

1 The Sun - an Introduction $\quad 4$

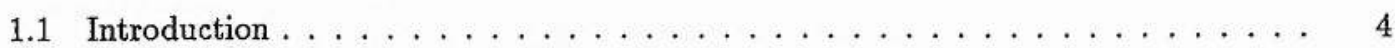

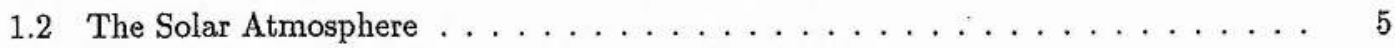

1.3 The Solar and Heliospheric Observatory $(\mathrm{SOHO}) \ldots \ldots \ldots \ldots \ldots$

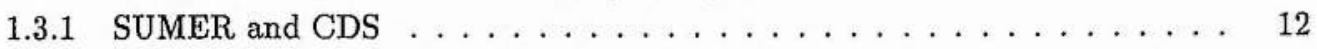

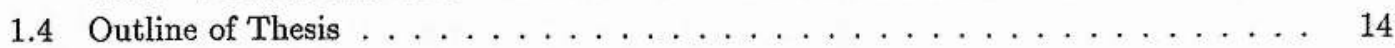

2 Basic Equations $\quad 15$

2.1 Maxwell's Equations . . . . . . . . . . . . . . . . . . . . . . . . 15

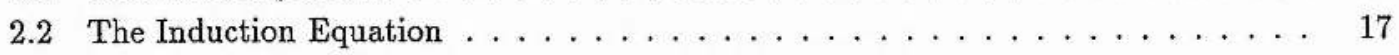

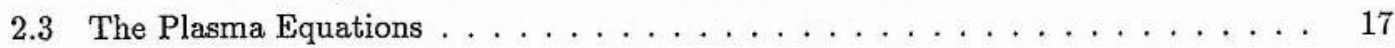

2.3.1 Mass Continuity . . . . . . . . . . . . . . . . . 17

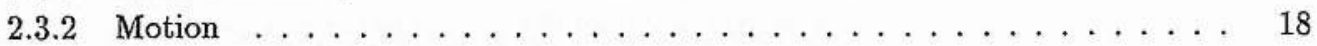

2.3 .3 The Energy Equation . . . . . . . . . . . . . . . . . . . . . . 18

2.3 .4 Perfect Gas Law . . . . . . . . . . . . . . . . . . . . . . . 19

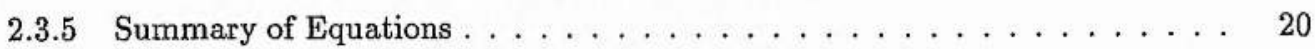

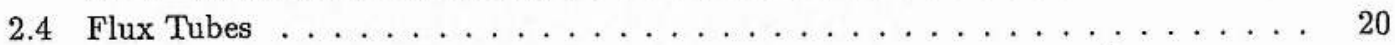

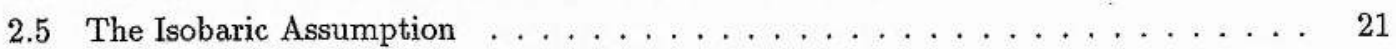

3 Thermal Equilibria of Coronal Loops 25

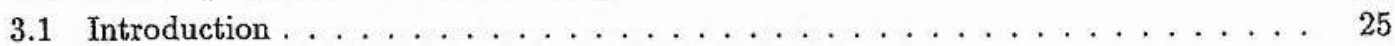

3.2 The Thermal Equilibrium Equation and a Simplified Radiative Loss Function . . . 30

3.3 Phase Plane Analysis . . . . . . . . . . . . . . . . . . . . 32

3.4 Analytical Solution for the Dependence of the Loop Summit Temperature upon Loop Length . . . . . . . . . . . . . . . . . . . . . . 35

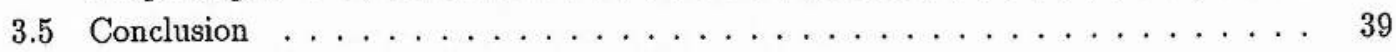

4 Time-Dependent Solar Coronal Heating 40

4.1 Introduction . . . . . . . . . . . . . . . . . . . . . 40

4.2 Heating Mechanisms in the Corona . . . . . . . . . . . . . . . . . . . . . 42

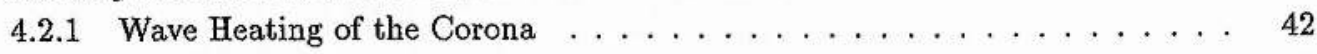

4.2 .2 MHD Turbulence . . . . . . . . . . . . . . . . . . 47

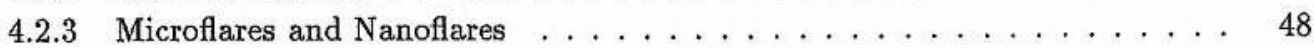

4.2 .4 Coronal Loop Interactions . . . . . . . . . . . . . . . . . . . . . 54 
4.2.5 Why Time-Dependent Heating? . . . . . . . . . . . . . . . 56

4.3 The Isobaric Equations and the Time-Dependent Heating Term . . . . . . . . . . 59

4.4 Analytical Test of Numerical Code for Time-Dependent Heating of an Initially Uniform Atmosphere . . . . . . . . . . . . . . . . . 60

4.5 Time-Dependent Heating of an Initially Static Loop . . . . . . . . . . . . . 61

4.6 Time-Dependent Heating of an Initially Static Loop including Hildner's Radiative Loss Function . . . . . . . . . . . . . . . . . . . . . T0

4.7 The Differential Emission Measure and Time-Dependent Heating . . . . . . . 71

4.7 .1 Background . . . . . . . . . . . . . . . . . 71

4.7.2 Time-Dependent Differential Emission Measure . . . . . . . . . . . . . 74

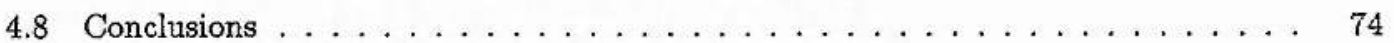

5 Other Forms of Time-Dependent Heating $\quad 77$

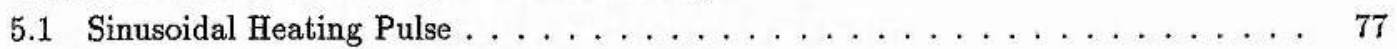

5.2 Triangular Heating Pulse $\ldots \ldots \ldots \ldots \ldots \ldots \ldots \ldots$

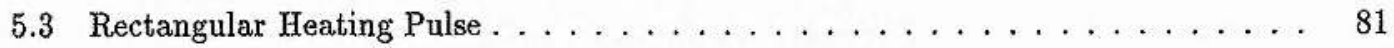

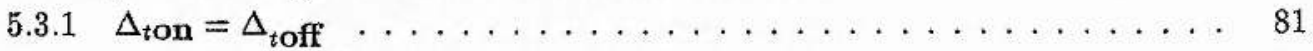

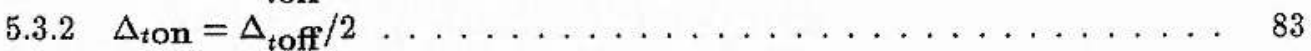

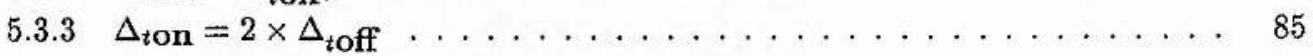

5.3.4 Summary of Rectangular Pulse Heating . . . . . . . . . . 85

5.4 Random Heating Pulse . . . . . . . . . . . . . . . . . . 85

5.4 .1 Fixed Time Interval . . . . . . . . . . . . . . . . 86

5.4 .2 Fixed Energy Quanta $\ldots \ldots \ldots \ldots \ldots \ldots \ldots$

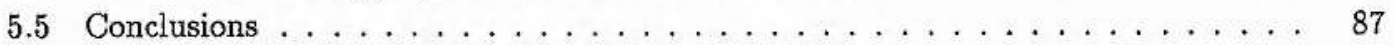

6 Validity of the Isobaric Assumption to the Corona 91

6.1 Introduction and Basic Equations . . . . . . . . . . . . . . . . 91

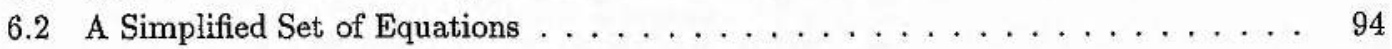

6.2 .1 Linearization of Equations . . . . . . . . . . . . . . . 95

6.2.2 Dependence of Dispersion Relation on $\epsilon_{0}$ and $b \ldots \ldots \ldots$. . . . 96

6.2.3 Numerical Calculation for the Decay of an Initially Perturbed Uniform At-

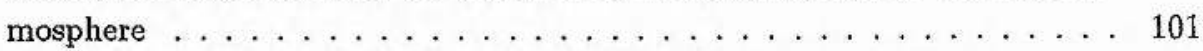

6.2 .4 Addition of Viscosity . . . . . . . . . . . . . . . . . . . . . . . . . . . . . . . . . 102

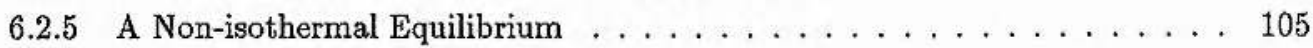

6.3 Time-Dependent Heating of an Initially Static Coronal Loop $\ldots \ldots \ldots \ldots \ldots$

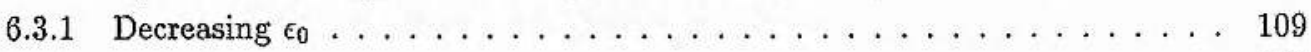

6.3 .2 Reducing $\omega_{\text {inert }} \ldots \ldots \ldots \ldots \ldots \ldots \ldots \ldots \ldots \ldots$

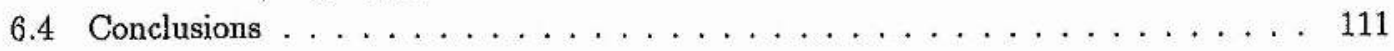

7 Summary and Further Work 113

7.1 Summary . . . . . . . . . . . . . . . . . . . 113

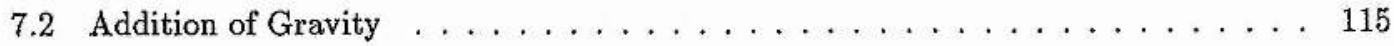

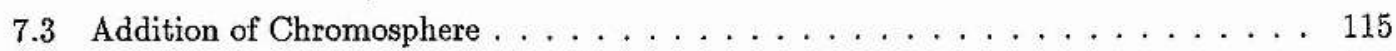

7.4 Variation in Loop Cross-Sectional Area . . . . . . . . . . . . . 116 
7.5 Random Heating Events . . . . . . . . . . . . . . . . 116

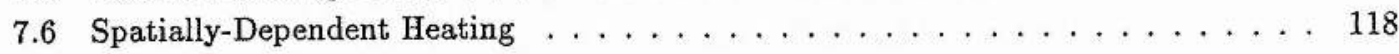

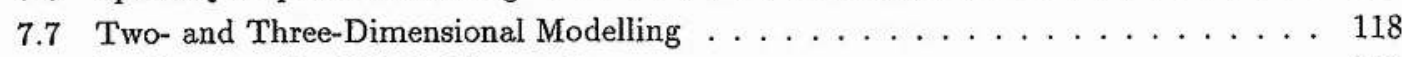

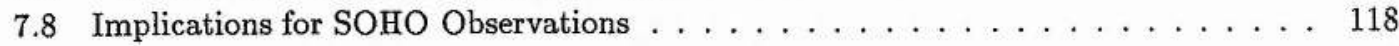

7.8 .1 SUMER and CDS Studies . . . . . . . . . . . . . . . . . . . . 119

7.8 .2 Possible Observations . . . . . . . . . . . . . . . . . . 120

A. Critical Point Analysis of an Autonomous System 121

B Analytical Calculation for the Dependence of The Summit Temperature upon Loop Length

C Derivation of $\mathrm{T}(\mathrm{x}, \mathrm{t})$ for Time-Dependent Heating with $\omega \gg 1$

D Analytical Calculation of Solutions to the Dispersion Relation 133

E Asymptotic Expansion of the Dispersion Relation 135

F Numerical Algorithm for Reduced Isobaric Equations $\quad 137$

G Numerical Algorithm for Inertial Equations $\quad 139$

$\begin{array}{lr}\text { Bibliography } & 141\end{array}$ 


\section{Chapter 1}

\section{The Sun - an Introduction}

- And God said, "Let there be light," and there was light.

God saw that the light was good.

(Genesis 1:3)

\subsection{Introduction}

The Sun is our nearest star and is the geometrical and gravitational centre of our planetary system. To the astronomer there is nothing exceptional about the Sun. However, it does dominate our skies and is the essential source of light, heat and energy for every living thing on Planet Earth. Our next nearest star lies approximately one quarter of a million times further away than the Sun and it takes light more than four years to travel to us from this celestial neighbour compared to eight minutes from the Sun.

Although the Sun is a stable and well-behaved star, relatively minor changes in its activity can have discernible effects on the Earth. Large eruptions of solar plasma towards the Earth (coronal mass ejections or CME's) have been known to disrupt powerlines and cause poor reception from telecommunication systems. Longer term changes in the output of the Sun may well be responsible for climatic changes on our planet (for example, the "Mini" Ice-Age between 1645-1715 when the Sun was at a sunspot minimum).

Figure 1.1 shows a sketch of the overall structure of the Sun while Table 1.1 presents some of its vital statistics. The solar interior with its core, radiative zone and convective zone, has been studied in detail by the powerful techniques of helioseismology (the study of solar surface oscillations) while the solar atmosphere (consisting of the photosphere, chromosphere and corona) is a magnetohydrodynamical laboratory where we can observe in detail many processes in which the 


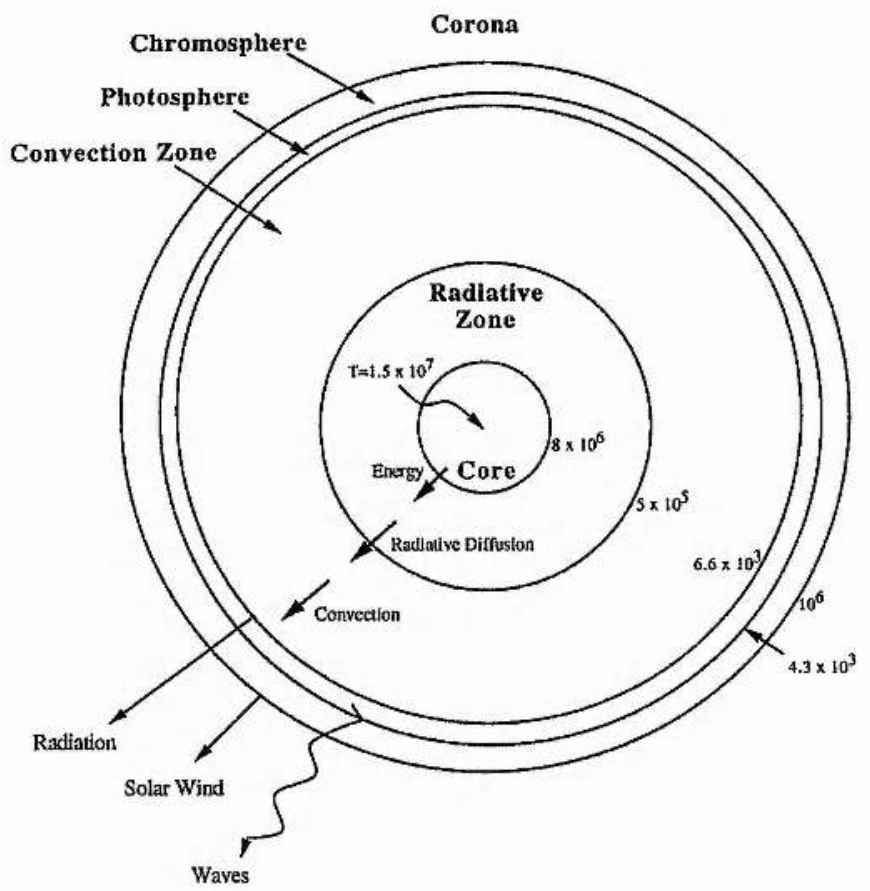

Figure 1.1: Overall structure of the solar interior (core, radiative zone and convection zone) and the solar atmosphere (photosphere, chromosphere and corona) indicating typical temperatures.

Sun's plasma and magnetic field interact. It is the outer atmosphere of the Sun with which this thesis is concerned.

\subsection{The Solar Atmosphere}

The solar atmosphere consists of three layers. The visible surface of the Sun is the photosphere which is only $\sim 0.5 \mathrm{Mm}$ wide. It has a temperature of $\sim 4200 \mathrm{~K}$ and number density of $10^{23} \mathrm{~m}^{-3}$ - the temperature decreases from the base to the top of the photosphere. Immediately above lies the rarer chromosphere $\left(10^{17} \mathrm{~m}^{-3}\right)$ within which the temperature rises slowly at first but then at $\sim 2 \mathrm{Mm}$, it increases suddenly to $\sim 2 \times 10^{6} \mathrm{~K}$ in the corona. This is the outermost part of the atmosphere and it extends out from the Sun and into interplanetary space, enveloping the Earth. This steep temperature rise into the corona is shown in Figure 1.2.

The Sun appears different at different levels. Across the photosphere appear dark spots (sunspots) - they are the result of the solar magnetic field poking up through from the solar interior. Since the photosphere sits on top of the convection zone, a granulation pattern is observed associated with large scale fluid motions. Figure 1.3 shows this process (as well as a large sunspot) where the brighter, central regions correspond to rising, hotter fluid while the darker, narrow lanes contain cooler, 


\begin{tabular}{|c|c|}
\hline Physical Parameters & Values \\
\hline Age & $4.5 \times 10^{9}$ years \\
Mass & $1.99 \times 10^{30} \mathrm{~kg}$ \\
Radius & $6.96 \times 10^{8} \mathrm{~m}$ \\
Mean Density & $1.4 \times 10^{3} \mathrm{~kg} \mathrm{~m}^{-3}$ \\
Mean Distance From Earth (1 AU) & $1.5 \times 10^{11} \mathrm{~m}$ \\
Surface Gravity & $274 \mathrm{~ms}^{-2}$ \\
Escape Velocity & $6.18 \times 10^{5} \mathrm{~m} \mathrm{~s}^{-1}$ \\
Equatorial Rotation Period & 26 days $^{-1}$ \\
Mass Loss Rate & $10^{9} \mathrm{~kg} \mathrm{~s}^{-1}$ \\
Effective Black Body Temperature & $5785 \mathrm{~K}$ \\
Inclination to Earth's orbit & $7^{\circ}$ \\
Composition & $90 \% \mathrm{H}, 10 \% \mathrm{He}$, \\
& $0.1 \%$ other elements $(\mathrm{C}, \mathrm{N}, \mathrm{O}, \ldots)$ \\
\hline
\end{tabular}

Table 1.1: Sun Statistics.

sinking material. Typical granulation speeds are of the order of a few kilometres per second.

The chromosphere can be revealed by observing the Sun through an $\mathrm{H} \alpha$ filter (Figure 1.4). Thin dark ribbons of cool plasma (known as filaments or prominences) are observed and the areas surrounding sunspots are brighter than anywhere else these are termed active regions.

Traditionally the corona was only visible at a solar eclipse. This occurs when the Moon crosses in front of the Earth such that its apparent diameter, when viewed from a certain point on the Earth's surface, obscures the solar disk and makes the corona visible. Figure 1.5 shows such an eclipse with the corona extending outwards from the solar surface over many solar radii. The most common structures are helmet streamers, bright elongated features that are wide near the solar limb but taper off to a long, narrow spike much further away from the solar surface. These streamers are thought to outline the magnetic field - at the base of the streamers there are nested magnetic fieldlines, anchored at both ends below the photosphere, but beyond a certain height, these loops "appear open", stretched radially outwards by the plasma streaming from the solar surface - the solar wind.

Thus, throughout history, coronal research was dominated by the fact that observations were only possible during this special astronomical circumstance of a total solar eclipse. However in about 1930, a French astronomer, Bernard Lyot, solved 


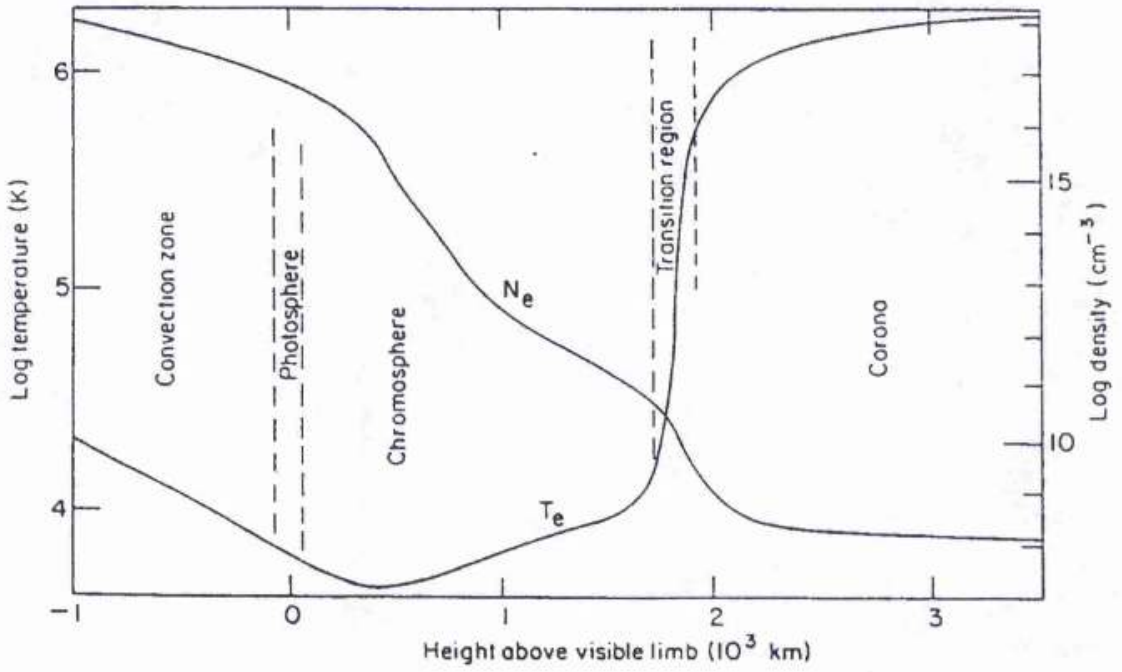

Figure 1.2: Schematic representation of the variation with height of the mean values of temperature and density in the outer layers of the Sun.

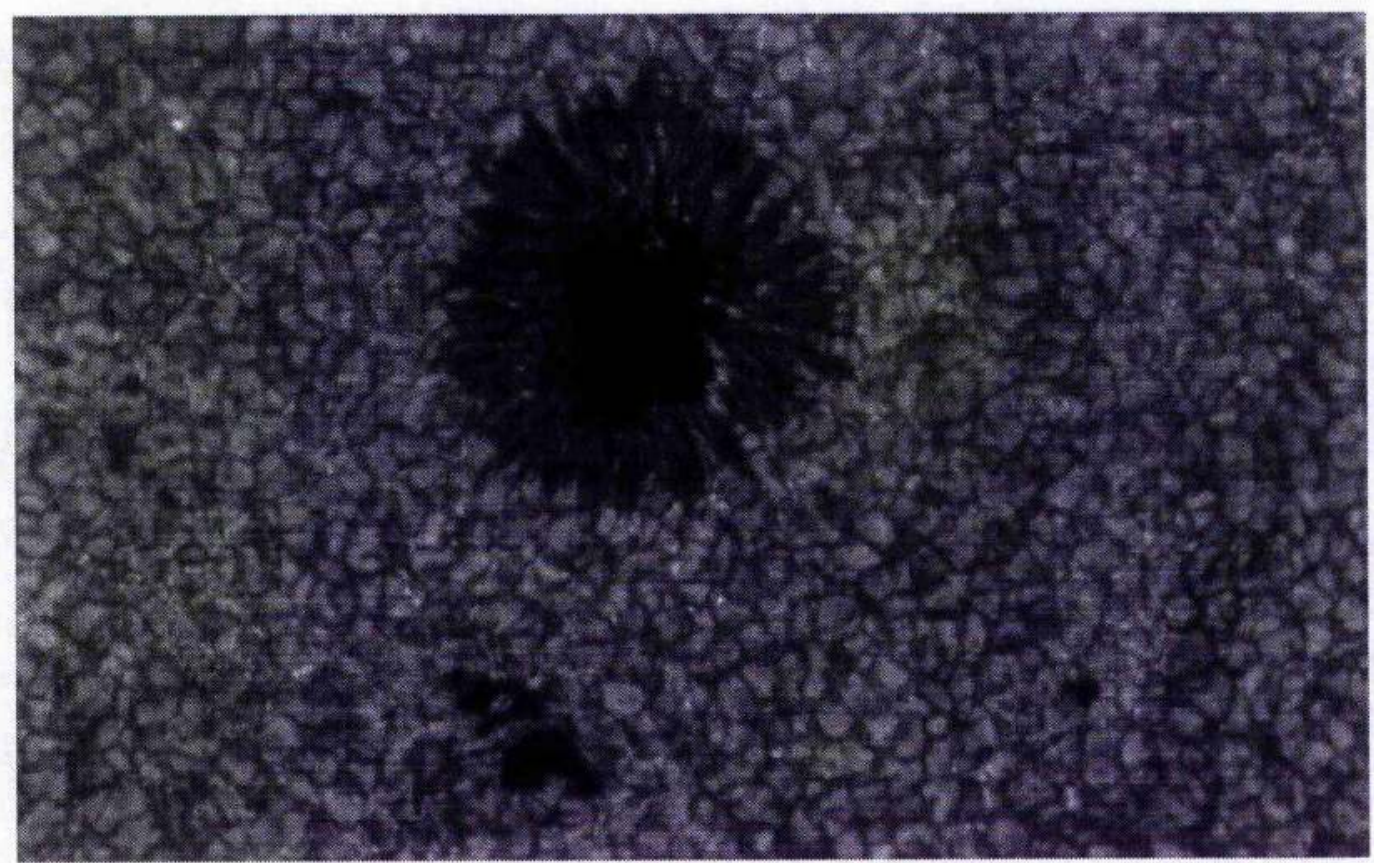

Figure 1.3: White light observation of a sunspot, clearly showing the granulation process in the photosphere (from the High Altitude Observatory, Boulder, Colorado). 


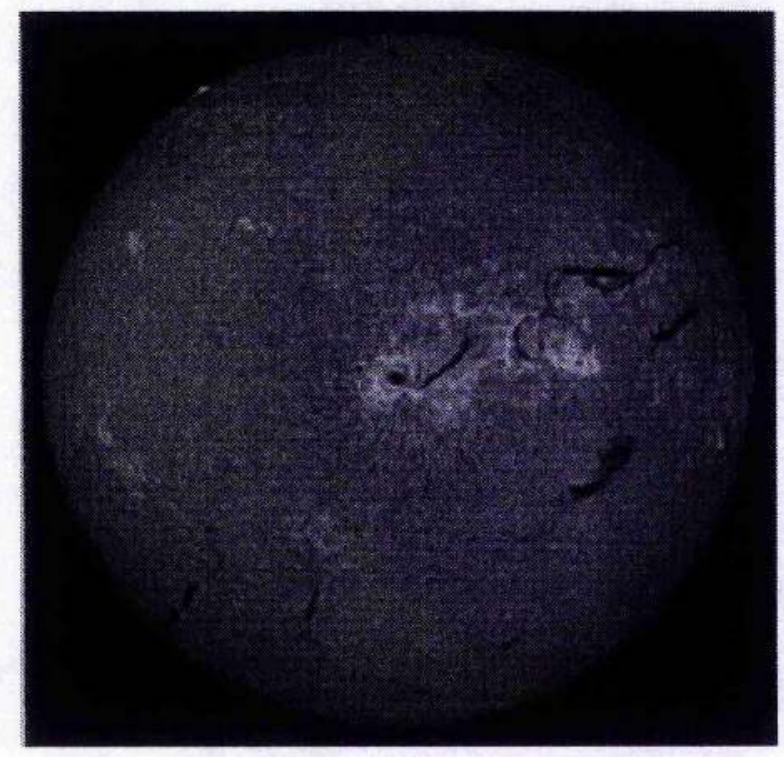

Figure 1.4: $\mathrm{H} \alpha$ image of the solar disk (from the Space Environment Laboratory, Boulder, Colorado).

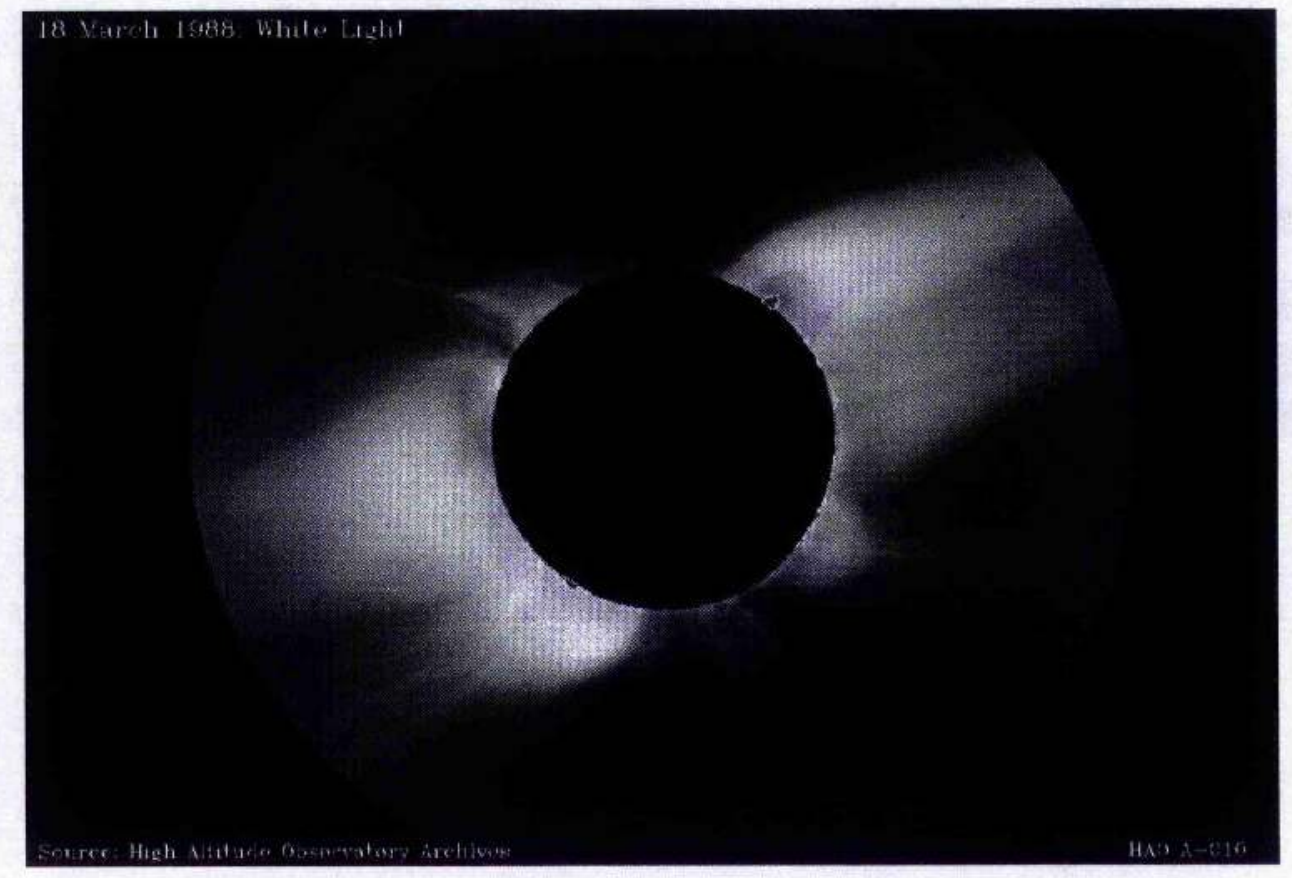

Figure 1.5: Total Eclipse of the Sun taken on $18^{\text {th }}$ March 1988 in the Philippines (from the High Altitude Observatory, Boulder, Colerado). 


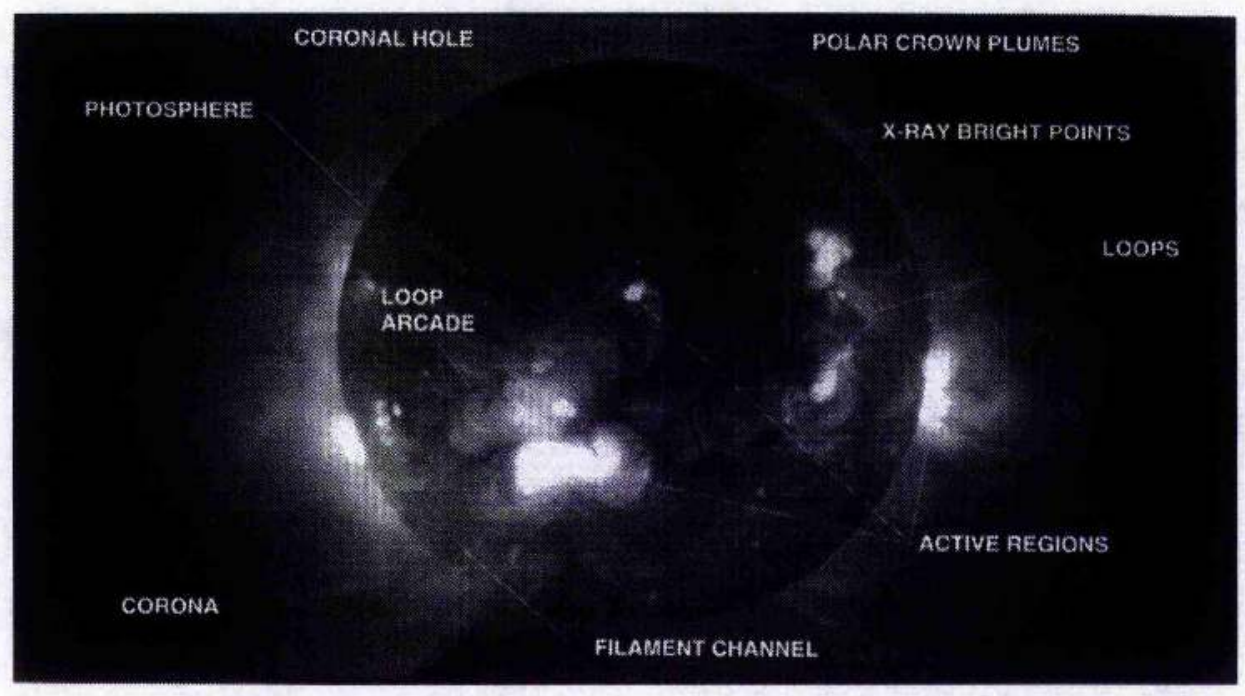

Figure 1.6: X-ray image of the Sun ( ${ }^{\text {th }}$ May, 1992) obtained from the Soft X-Ray Telescope on-board Yohkoh (from NASA).

the problem by creating an artificial eclipse within a telescope system (a coronagraph), therefore allowing the corona to be observed on a much more regular basis. Our understanding and respect for the corona was completely transformed by $\mathrm{X}$ ray observations initially from the Skylab and Solar Maximum Missions and more recently by the Yohkoh satellite. With a "surface" temperature of $\sim 5800 \mathrm{~K}$, the Sun should not normally emit X-rays and should therefore look completely dark on an X-ray image. This is not the case. While portions of the Sun are indeed dark, very bright regions are also present. Figure 1.6 shows the highly structured and dynamic atmosphere produced by the interaction of the plasma with the magnetic field. Figure 1.7 shows the same solar image but in the form of a magnetogram - a synthetic picture constructed by measuring the magnetic field along the line of sight at a given location on the solar surface. The region of positive and negative polarity shown in Figure 1.7 corresponds to the largest active region in the south-west corner of the disc in Figure 1.6.

These soft X-ray images reveal a new world of X-ray bright points (thought to be sites of magnetic reconnection), coronal holes (darker regions where the magnetic field is open and along which the majority of the solar wind is streaming outwards) and coronal loops (magnetic loops containing plasma). Images such as Figure 1.6 can be used to construct models of the distribution of temperature in 


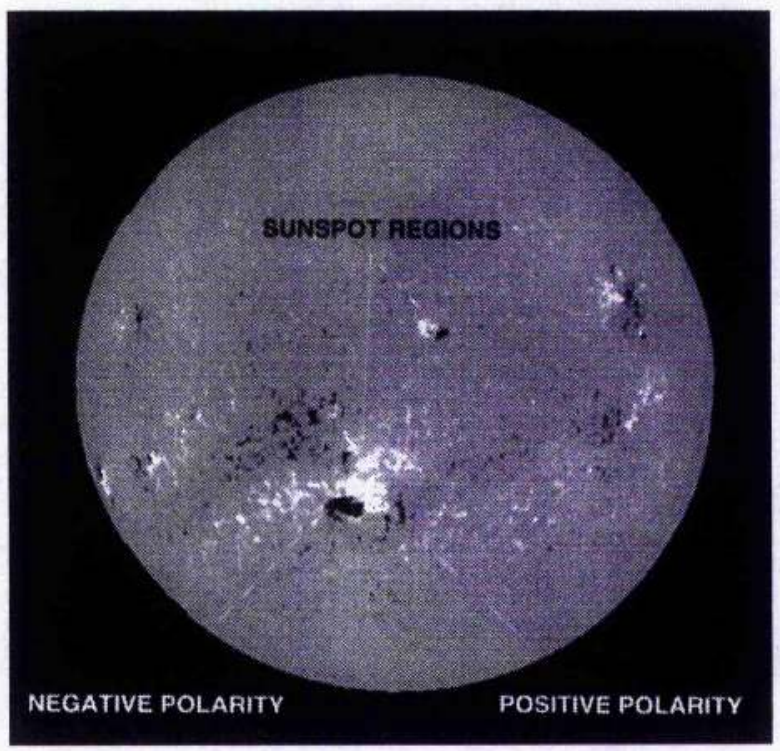

Figure 1.7: Magnetogram of Sun, $8^{\text {th }}$ May, 1992 (from the Big Bear Observatory).

the corona, investigate the heating mechanism and determine the size and physical conditions of many coronal features.

Figure 1.8 shows a portion of the solar disk containing coronal loop structures. It is these particular features which we will be investigating further by trying to model their physical properties in response to a time-varying coronal heating supply.

\subsection{The Solar and Heliospheric Observatory (SOHO)}

The Solar and Heliospheric Observatory (SOHO) is one of the ESA and NASA's most ambitious projects to date. SOHO is a space-based observatory that is totally dedicated to furthering our understanding of the Sun. It has three principle objectives;

- Why does the solar corona exist and how is it heated?

- How are the solar wind streams accelerated?

- What is the nature of the solar interior?

Launched late in 1995, it will be stationed in a halo orbit around the L1 Lagrangian point approximately $1.5 \times 10^{6} \mathrm{~km}$ from the Earth where the gravitational pull of the Sun and the Earth are equal. In this position, it will provide an uninterrupted view 


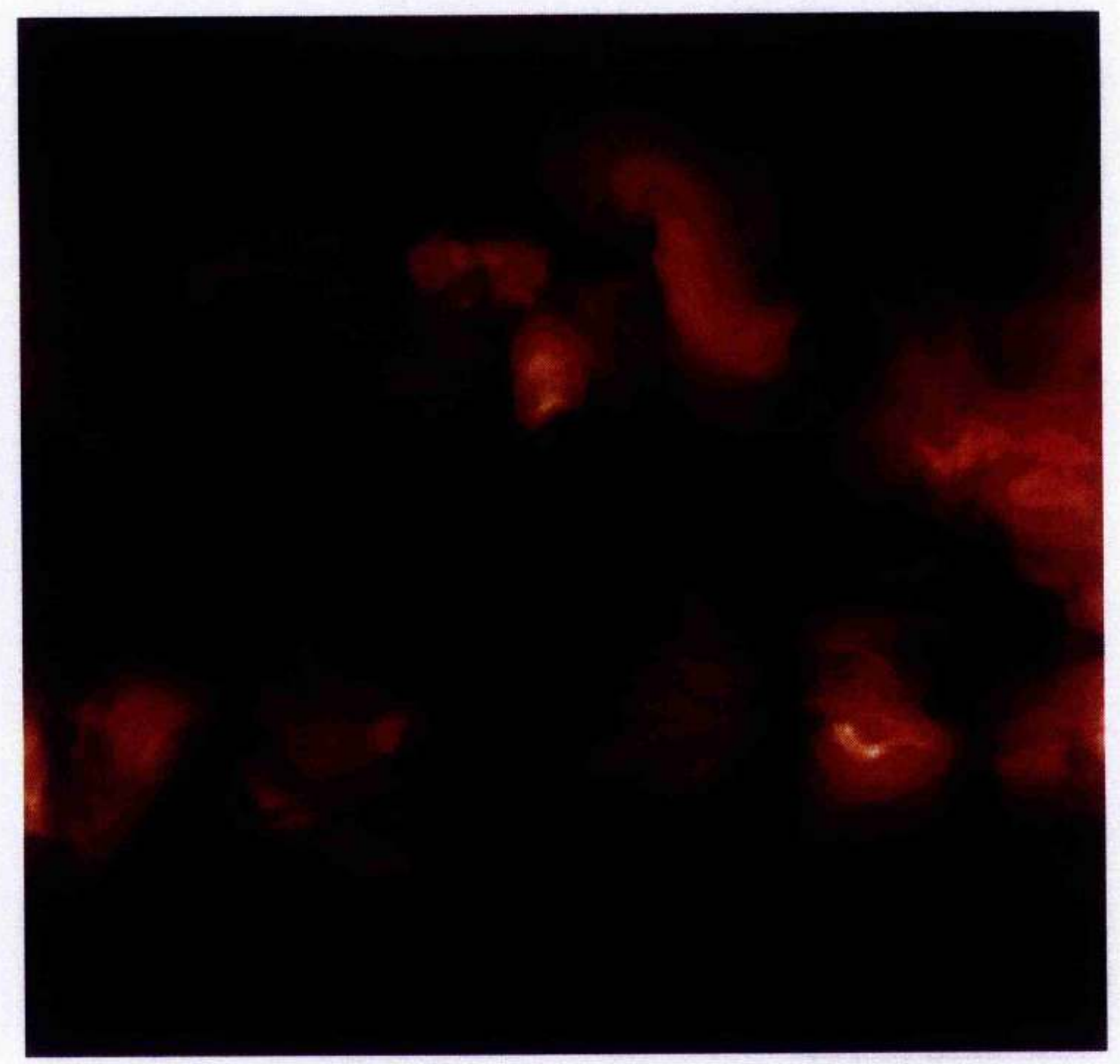

Figure 1.8: Yohkoh Soft X-ray Telescope image of coronal loop structures (from J. Klimchuk). 


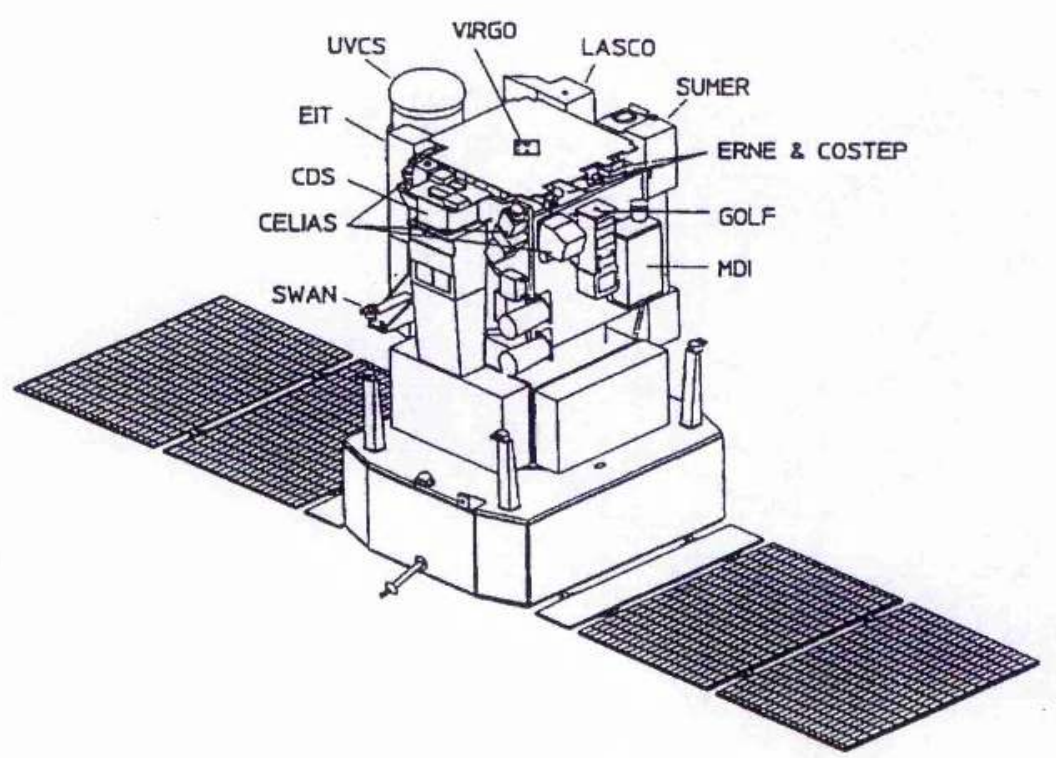

Figure 1.9: Schematic view of SOHO.

of the Sun, sitting outside the magnetosphere and therefore well within the solar wind streams. The scientific payload of SOHO consists of twelve sets of experiments as listed in Table 1.2 (Harrison and Fludra, 1995). Figure 1.9 shows a schematic view of the satellite and the relative positions of the different instruments. The experiments can be divided into three main groups according to their particular area of research: helioseismology, solar corona and solar wind "in situ" instruments (Domingo et al, 1994; Mason, 1994). We will be concerned with the remote sensing investigations of the solar atmosphere.

\subsubsection{SUMER and CDS}

Investigations of the corona will be carried out with a set of telescopes and spectrometers that will produce the data necessary to study the dynamic processes that are taking place there. In particular, SUMER (Solar Ultraviolet Measurements of Emitted Radiation) (500 - $1600 \AA$ ) will be equipped to study plasma flows, temperatures, densities and wave motions in the upper chromosphere, transition region and lower corona while CDS (Coronal Diagnostic Spectrometer) (160-800 $\AA$ ) will determine the physical parameters of various coronal structures at slightly higher temperatures. Both of these instruments should be able to recognise particular signatures within the corona in order to evaluate the relevance of the different models for coronal heating. These instruments complement each other very well and many joint observing sequences have been planned (Harrison and Fludra, 1995; Wilhelm, 


\begin{tabular}{|c|c|}
\hline Experiment & Description \\
\hline $\begin{array}{c}\text { GOLF - } \\
\text { Global Oscillations at } \\
\text { Low Frequencies }\end{array}$ & $\begin{array}{l}\text { Global Sun velocity and } \\
\text { magnetic field oscillations }\end{array}$ \\
\hline $\begin{array}{c}\text { VIRGO - } \\
\text { Variability of Solar } \\
\text { Irradiance }\end{array}$ & $\begin{array}{l}\text { Global and low resolution } \\
\text { imaging of oscillations and } \\
\text { solar constant }\end{array}$ \\
\hline $\begin{array}{c}\text { MDI - } \\
\text { Michelson Doppler } \\
\text { Imager }\end{array}$ & $\begin{array}{l}\text { High resolution imaging of } \\
\text { velocity oscillation }\end{array}$ \\
\hline $\begin{array}{c}\text { SUMER - } \\
\text { Solar UV Measurement } \\
\text { of Emitted Radiation }\end{array}$ & $\begin{array}{c}\text { Plasma flows, temperature } \\
\text { and density in the solar } \\
\text { atmosphere }\end{array}$ \\
\hline $\begin{array}{c}\text { CDS - } \\
\text { Coronal Diagnostic } \\
\text { Spectrometer }\end{array}$ & $\begin{array}{c}\text { Density, temperature and } \\
\text { flows in the solar } \\
\text { atmosphere }\end{array}$ \\
\hline $\begin{array}{c}\text { EIT - } \\
\text { Extreme UV Imaging } \\
\text { Telescope }\end{array}$ & $\begin{array}{l}\text { Evolution of low coronal } \\
\text { structure and activity }\end{array}$ \\
\hline $\begin{array}{l}\text { UVCS - } \\
\text { UV Coronagraph and } \\
\text { Spectrometer }\end{array}$ & $\begin{array}{l}\text { Density and temperature } \\
\text { in the corona }\end{array}$ \\
\hline $\begin{array}{l}\text { LASCO - } \\
\text { Large Angle Spectrometer } \\
\text { Coronagraph }\end{array}$ & $\begin{array}{c}\text { Structure and evolution of } \\
\text { the corona }\end{array}$ \\
\hline $\begin{array}{c}\text { SWAN - } \\
\text { Solar Wind Anisotropies }\end{array}$ & $\begin{array}{l}\text { Solar wind mass flux } \\
\text { anisotropies }\end{array}$ \\
\hline $\begin{array}{c}\text { CELIAS - } \\
\text { Charge, Element, Isotope } \\
\text { Analysis }\end{array}$ & $\begin{array}{l}\text { Ion composition in the } \\
\text { solar wind }\end{array}$ \\
\hline $\begin{array}{c}\text { COSTEP - } \\
\text { Suprathermal and Energetic } \\
\text { Particle Analyser }\end{array}$ & $\begin{array}{l}\text { Ion and electron } \\
\text { composition in the solar } \\
\text { wind }\end{array}$ \\
\hline $\begin{array}{c}\text { ERNE - } \\
\text { Energetic Particle Analyser }\end{array}$ & $\begin{array}{c}\text { Ion and electron } \\
\text { composition in the solar } \\
\text { wind }\end{array}$ \\
\hline
\end{tabular}

Table 1.2: The SOHO Payload. 
1995). Possible sequences of observations relating to coronal heating and loop structures that are relevant to this thesis can be found in Section 7.8.

Thus, to summarize, the SOHO satellite is an unprecedented opportunity for the Solar Community to study in great detail the structure, chemical composition and dynamics of the solar interior, solar atmosphere and solar wind.

\subsection{Outline of Thesis}

The work contained within this thesis is primarily concerned with modelling the heating of coronal loops. Previous work by various authors on the thermal equilibria of these magnetic structures can be found in Section 3.1 while overviews of the proposed heating mechanisms for the corona are outlined in Sections 4.2.1 to 4.2.4. It is not the aim of this work to investigate a particular heating method but rather to understand the response of the plasma to a time-varying supply of heat energy. A time-dependent heating term is required because it is likely that none of the suggested heating mechanisms can provide a continual, constant supply of heat.

A short review of the magnetohydrodynamic equations is given in Chapter 2, particularly in respect to modelling coronal loops. An isobaric assumption is also introduced which allows one to follow the thermal evolution without the restriction of tracking the propagation of sound waves in the system.

A detailed understanding of the behaviour of any physical system requires an understanding of the possible static solutions and in the case of coronal loops, this is generally a complicated problem due to the form of the radiation. However, by using a simplified radiative loss function, it is possible to derive an analytical solution for these equilibrium states (Chapter 3 ).

In Chapter 4 the numerical results for the response of a typical coronal loop to a sinusoidal time variation in the heating are presented. The frequency of the heat deposited is investigated from which some conclusions are drawn on the nature of a time-dependent heating source in the corona. Much of the work in this chapter has been accepted for publication in Walsh et al (1995a, 1995b). Chapter 5 extends the work to other forms of heating including random energy releases on the scale of nanoflares. The validity of the isobaric assumption to coronal loops is investigated in Chapter 6 by including the inertial terms into the system of equations.

A short summary as well as a description of possible further work is given in Chapter 7. Finally, a brief outline of the numerical algorithms used can be found in Appendices $\mathrm{F}$ and $\mathrm{G}$ (Walsh et $a l, 1995 \mathrm{c}$ ). 


\section{Chapter 2}

\section{Basic Equations}

"There' is more to visualising than the formulae", Audrea said, "just as there is more to a symphony than the mathematical relationship between the notes. The formulae are simply aids that point out certain essential relationships pulling them, so to speak, out of the ocean of other lesser relationships, the way a musician identifies and isolates major themes and structures".

(from 'Probe' by M.W. Bonanno)

Magnetohydrodynamics is the study of the interaction between a magnetic field and a plasma using a simplified set of Maxwell's equations along with Ohm's Law, the Gas Law and equations for mass continuity, motion and energy.

\subsection{Maxwell's Equations}

These are

$$
\begin{aligned}
\nabla \times \mathbf{B} & =\mu \mathbf{j}+\frac{1}{c^{2}} \frac{\partial \mathbf{E}}{\partial t} \\
\nabla \cdot \mathbf{B} & =0 \\
\nabla \times \mathbf{E} & =-\frac{\partial \mathbf{B}}{\partial t} \\
\nabla . \mathbf{E} & =\frac{\rho_{e}}{\varepsilon}
\end{aligned}
$$

where $\mathbf{E}$ is the electric field strength, $\mathbf{B}$ is the magnetic field strength, $\rho_{e}$ is the charge density, $\mathbf{j}$ is the current density, $\mu$ is the magnetic permeability, $\varepsilon$ is the permittivity of free space, $t$ is the time and $c$ is the speed of light in a vacuum $\left(\sim 3 \times 10^{8} \mathrm{~ms}^{-1}\right)$. These equations are simplified under certain assumptions (Priest, 1982): 
- The plasma is treated as a continuous medium (valid as long as the lengthscales considered greatly exceed any internal plasma lengthscales, for example, the ion gyroradius).

- The plasma is assumed to be in thermal equilibrium.

- $\mu$ and $\varepsilon$ are assumed to be constant (and taken to be the vacuum values $\mu_{0}=$ $4 \pi \times 10^{-7} \mathrm{Hm}^{-1}$ and $\varepsilon_{0}=8.854 \times 10^{-12} \mathrm{Fm}^{-1}$ in the Solar context).

- Most of the other plasma properties are supposed to be isotropic except the thermal conduction, $\kappa$, which is much greater along the magnetic field direction than perpendicular to it (see Section 2.3.3).

- In spite of the fact that the Sun is a rotating body, an inertial frame of reference is used. Rotational effects may become important when considering very large structures.

- The plasma is treated as a single fluid system.

- A simplified Ohm's Law can be applied;

$$
\mathrm{j}=\sigma(\mathbf{E}+\mathbf{v} \times \mathbf{B})
$$

where $\mathrm{v}$ is the plasma velocity and $\sigma$ is the electrical conductivity.

- Flow, sound and Alfvénic velocities are much smaller than the speed of light and therefore relativistic effects can be ignored. Thus, if we consider

$$
v_{0}=l_{0} / t_{0}
$$

to be a typical plasma speed where $l_{0}$ and $t_{0}$ are a typical plasma length and timescale respectively and, in addition, if we assume from equation (2.3) that

$$
\frac{E_{0}}{l_{0}} \approx \frac{B_{0}}{t_{0}}
$$

where $E_{0}$ and $B_{0}$ are typical values for $\mathbf{E}$ and $\mathbf{B}$, then by comparing sizes of terms in equation (2.1), the second term on the right-hand side has magnitude

$$
\frac{E_{0}}{c^{2} t_{0}} \approx \frac{B_{0} l_{0}}{c^{2} t_{0}^{2}}=\frac{v_{0}^{2} B_{0}}{c^{2} l_{0}} \approx \frac{v_{0}^{2}}{c^{2}}|\nabla \times \mathbf{B}|
$$

which is smaller than the left-hand side of $(2.1)$ as $v_{0} \ll c$. Thus, the term $\partial \mathbf{E} / \partial t$ can be neglected, that is

$$
\nabla \times \mathbf{B}=\mu_{0} \mathbf{j} .
$$




\subsection{The Induction Equation}

Using equation (2.5) to eliminate $\mathbf{E}$ from equation (2.3), we get

$$
\frac{\partial \mathbf{B}}{\partial t}=-\nabla \times\left(\frac{\mathbf{j}}{\sigma}-\mathbf{v} \times \mathbf{B}\right),
$$

and with equation (2.6),

$$
\frac{\partial \mathbf{B}}{\partial t}=-\nabla \times\left(\frac{1}{\mu_{0} \sigma} \nabla \times \mathbf{B}-\mathbf{v} \times \mathbf{B}\right) .
$$

Using the triple vector product,

$$
\nabla \times(\nabla \times \mathbf{B})=\nabla(\nabla . \mathbf{B})-\nabla^{2} \mathbf{B}=-\nabla^{2} \mathbf{B},
$$

from equation (2.2), equation (2.8) reads

$$
\frac{\partial \mathbf{B}}{\partial t}=\nabla \times(\mathbf{v} \times \mathbf{B})+\eta_{0} \nabla^{2} \mathbf{B}
$$

where $\eta_{0}=\left(\mu_{0} \sigma\right)^{-1}$ is the constant magnetic diffusivity. Equation (2.9) is known as the Induction Equation and it links the evolution of the magnetic field to the plasma.

\subsection{The Plasma Equations}

The motion of the magnetic field is coupled to the behaviour of the plasma by the presence of the velocity term in equation (2.9) and in the following equations for mass continuity, motion and energy.

\subsubsection{Mass Continuity}

In an MHD system, mass must be conserved;

$$
\frac{D \rho}{D t}+\rho \nabla \cdot \mathrm{v}=0
$$

where

$$
\frac{D}{D t}=\frac{\partial}{\partial t}+\mathrm{v} \cdot \nabla
$$

is the total derivative and $\rho$ is the plasma density. 


\subsubsection{Motion}

The equation of motion for the plasma can be written as

$$
\rho \frac{D \mathbf{v}}{D t}=-\nabla p+\mathbf{j} \times \mathbf{B}+\rho \mathbf{g}+\rho \nu \nabla^{2} \mathbf{v},
$$

where $p$ is the plasma pressure. The terms on the right hand side of the equation can be separated into :

- $\nabla p$ - a plasma pressure gradient.

- $\mathbf{j} \times \mathbf{B}-\mathrm{a}$ Lorentz force. From equation (2.6),

$$
\mathbf{j} \times \mathbf{B}=\frac{1}{\mu_{0}}(\nabla \times \mathbf{B}) \times \mathbf{B}
$$

which, using the triple vector product, becomes

$$
\text { (B. } \nabla) \frac{\mathbf{B}}{\mu_{0}}-\nabla\left(\frac{B^{2}}{2 \mu_{0}}\right) \text {. }
$$

The first of these terms represents the change of $\mathbf{B}$ along a particular field line and therefore is a magnetic tension force whose strength is proportional to $B^{2}$. The second term is a magnetic pressure force with the magnetic pressure given by $B^{2} / 2 \mu_{0}$.

- $\rho \mathrm{g}$ - this is the effect of gravity where $\mathbf{g}$ is the local gravitational acceleration at the surface of the Sun (taken to be $\sim 274 \mathrm{~ms}^{-2}$ ).

- $\rho \nu \nabla^{2} \mathrm{v}$ - this is the effect of viscosity on an incompressible flow. $\nu$ is the coefficient of kinematic viscosity which is assumed to be uniform throughout the plasma and Spitzer (1962) gives

$$
\rho \nu=2.21 \times 10^{-16} \frac{T^{5 / 2}}{\operatorname{Ln} \Lambda} \mathrm{kgm}^{-1} \mathrm{~s}^{-1},
$$

where $\operatorname{Ln} \Lambda$ is the Coulomb Logarithm which we will take to be $\sim 20$ for the corona.

\subsubsection{The Energy Equation}

The fundamental energy equation is written as

$$
\frac{\rho^{\gamma}}{\gamma-1} \frac{D}{D t}\left(\frac{p}{\rho^{\gamma}}\right)=\nabla \cdot(K \nabla T)-L
$$


where $T$ is the plasma temperature and $\gamma$ is the ratio of specific heats $(=5 / 3) . K$ is the tensor of thermal conduction which can be split into components along and across the magnetic field,

$$
\nabla_{\| \cdot} \cdot\left(\kappa_{\|} \nabla_{\|} T\right)+\nabla_{\perp \cdot}\left(\kappa_{\perp} \nabla_{\perp} T\right)
$$

Along the magnetic fieldlines conduction is mainly by electrons and Braginski (1965) gives $\kappa_{\|}=\kappa_{0} T^{5 / 2} \mathrm{~W} \mathrm{~m}^{-1} \mathrm{deg}^{-1}$ with $\kappa_{0}=10^{-11}$ for the corona. Conduction across the fieldlines is mainly by ions and at coronal temperatures,

$$
\frac{\kappa_{\perp}}{\kappa_{\|}} \approx 10^{-12} \text {. }
$$

Thus, for the Sun with its strong magnetic field, a good approximation is that the vast majority of conducted heat occurs along the field and thus the conduction term can simply be written as

$$
\nabla_{\|} \cdot\left(\kappa_{\|} \nabla_{\|} T\right)=\kappa_{0} \nabla_{\|} \cdot\left(T^{5 / 2} \nabla_{\|} T\right) .
$$

$L$ is the loss-gain function which has the form

$$
L(\rho, T)=\rho^{2} Q(T)-H,
$$

where $Q(T)$ is the optically thin radiative loss function and $H$ is the unknown coronal heating function. $Q(T)$ has been calculated by several authors and is approximated by a piecewise continuous function,

$$
Q(T)=\chi T^{\alpha}
$$

where $\chi$ and $\alpha$ are constants within any particular range of temperature for the piecewise fit. It is shown in Figure 2.1 for Hildner (1974), Rosner et al (1978) and Cook et al (1989). The different heating theories and forms of $H$ will be discussed in Chapter 4.

\subsubsection{Perfect Gas Law}

For simplicity, the perfect gas law will be used;

$$
p=\frac{R}{\tilde{\mu}} \rho T,
$$

where $R$ is the molar gas constant $\left(8.3 \times 10^{3} \mathrm{~m}^{2} \mathrm{~s}^{-2} \mathrm{deg}^{-1}\right)$ and $\tilde{\mu}$ is the mean molecular weight with $\tilde{\mu}=0.6$ in the ionised corona. 


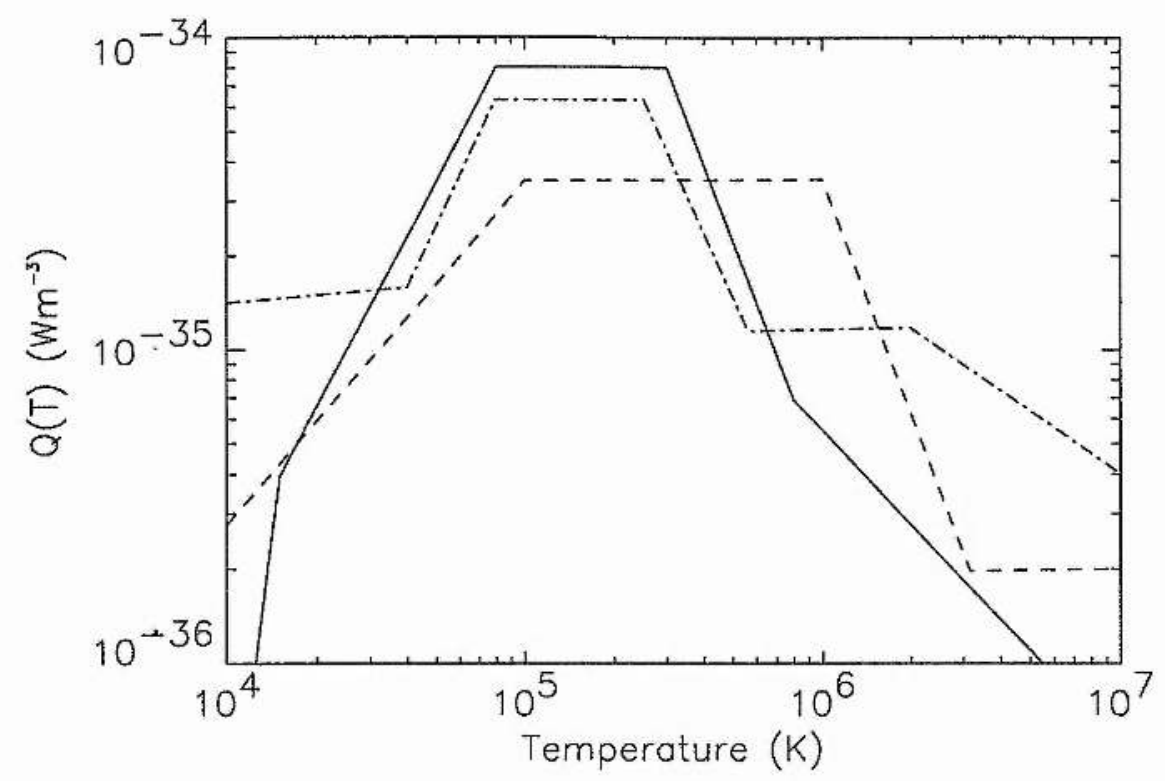

Figure 2.1: Comparison of (-) Hildner, 1974, (.-..) Rosner et al, 1978 and (--) Cook et al, 1989 profiles for the radiative loss function

\subsubsection{Summary of Equations}

Thus, the fundamental equations considered throughout this thesis are

$$
\begin{aligned}
\frac{\partial \mathbf{B}}{\partial t} & =\nabla \times(\mathbf{v} \times \mathbf{B})+\eta_{0} \nabla^{2} \mathbf{B} \\
\frac{D \rho}{D t}+\rho \nabla \cdot \mathbf{v} & =0 \\
\rho \frac{D \mathbf{v}}{D t} & =-\nabla p+\mathbf{j} \times \mathbf{B}+\rho \mathbf{g}+\rho \nu \nabla^{2} \mathbf{v} \\
\frac{\rho^{\gamma}}{\gamma-1} \frac{D}{D t}\left(\frac{p}{\rho^{\gamma}}\right) & =\kappa_{0} \nabla_{\| \cdot} \cdot\left(T^{5 / 2} \nabla_{\|} T\right)-\rho^{2} \chi T^{\alpha}+H \\
p & =\frac{R}{\tilde{\mu}} \rho T .
\end{aligned}
$$

\subsection{Flux Tubes}

A magnetic flux tube is the volume generated by the set of fieldlines which intersect a simple, closed curve (see Figure 2.2). The strength $F_{s}$ of the flux tube is the amount of magnetic flux $\mathbf{B}$ crossing a particular section, $S_{1}$, of the tube;

$$
F_{s}=\int_{s_{1}} \mathbf{B} \cdot d \mathbf{S}=\int_{S_{1}} B_{n} d S
$$




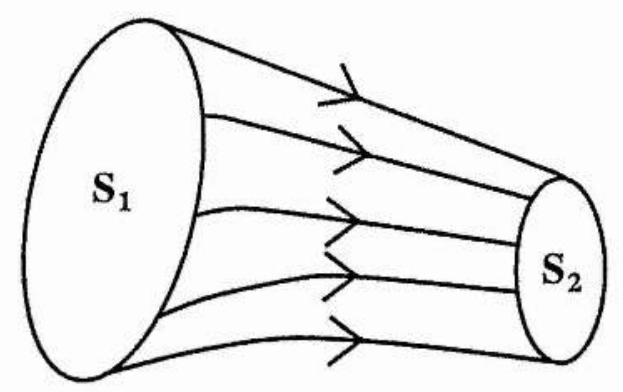

Figure 2.2: An example of a magnetic flux tube through surfaces $S_{1}$ and $S_{2}$.

where $B_{n}$ is the normal component of the field through section $S_{1}$.

The surface of the Sun is covered in coronal loops of various sizes and these outline the magnetic field as it rises up through the dense photosphere and chromosphere and spreads out in the rarefied corona (Figure 2.3). A coronal loop is therefore an example of a flux tube. It will be assumed that the coronal loops considered in this work have a constant cross-sectional area and that the magnetic field is so strong (that is, the coronal plasma $\beta$ is so small) that the loop structure remains rigid.

This type of magnetic channelling means that the induction equation (2.9) is no longer required and that the $\mathbf{j} \times \mathbf{B}$ force in equation (2.19) is identically zero. All plasma motions will be assumed to be along the coronal loop length, thus allowing us to restrict our investigations to one-dimensional dynamics. Equations (2.18) to (2.21) now read as

$$
\begin{aligned}
\frac{D \rho}{D t}+\rho \frac{\partial v}{\partial x} & =0 \\
\rho \frac{D v}{D t} & =-\frac{\partial p}{\partial x}+\rho g+\rho \nu \frac{\partial^{2} v}{\partial x^{2}} \\
\frac{\rho^{\gamma}}{\gamma-1} \frac{D}{D t}\left(\frac{p}{\rho^{\gamma}}\right) & =\kappa_{0} \frac{\partial}{\partial x}\left(T^{5 / 2} \frac{\partial T}{\partial x}\right)-\rho^{2} \chi T^{\alpha}+H \\
p & =\frac{R}{\tilde{\mu}} \rho T
\end{aligned}
$$

where all quantities are along the magnetic field and $x$ is the distance along a field line.

\subsection{The Isobaric Assumption}

The following derivation is similar to that outlined in Meerson(1989). Using equations (2.23) - (2.26) in which gravity and viscosity are neglected, consider writing 


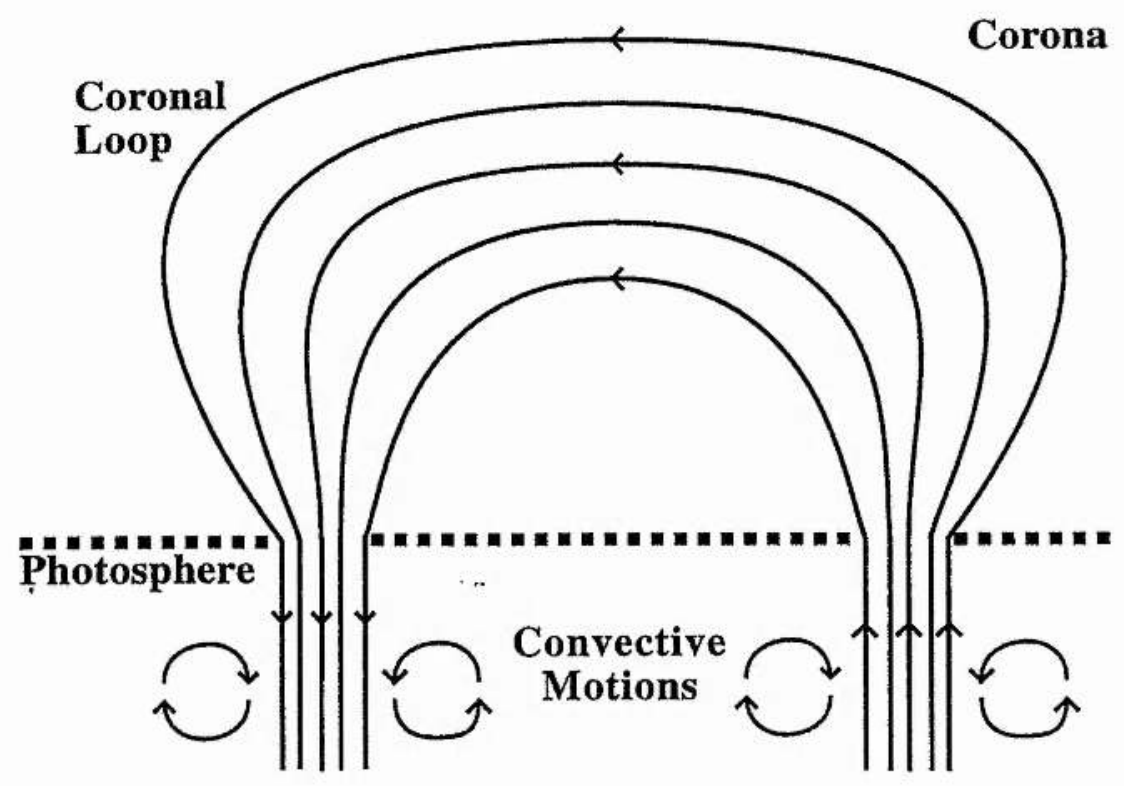

Figure 2.3: A schematic diagram of a coronal loop.

the mass continuity equation (2.23) as

$$
\frac{\partial \rho}{\partial t}+v \frac{\partial \rho}{\partial x}+\rho \frac{\partial v}{\partial x}=0
$$

therefore,

$$
\frac{\partial \rho}{\partial t}+\frac{\partial(\rho v)}{\partial x}=0
$$

and for the equation of motion (2.24),

$$
\rho\left(\frac{\partial v}{\partial t}+v \frac{\partial v}{\partial x}\right)=-\frac{\partial p}{\partial x}
$$

while for the energy equation (2.25), the left hand side can be expressed as

$$
\begin{aligned}
\frac{\rho^{\gamma}}{\gamma-1} \frac{D}{D t}\left(\frac{p}{\rho^{\gamma}}\right) & =\frac{1}{\gamma-1} \frac{D p}{D t}-\frac{\gamma p}{(\gamma-1) \rho} \frac{D \rho}{D t} \\
& =\frac{1}{\gamma-1} \frac{D p}{D t}+\frac{\gamma p}{(\gamma-1)} \frac{\partial v}{\partial x} \quad \text { (from (2.23)) } \\
& =\frac{1}{\gamma-1}\left(\frac{\partial p}{\partial t}+v \frac{\partial p}{\partial x}\right)+\frac{\gamma p}{(\gamma-1)} \frac{\partial v}{\partial x}
\end{aligned}
$$

by expanding the total derivative. The resulting equations can be made dimensionless against typical coronal values by setting

$$
x=l \bar{x}, \quad t=t_{c} \bar{t}, \quad v=v_{c} \bar{v}=\frac{l \bar{v}}{t_{c}}, \quad T=T_{c} \bar{T}, \quad \rho=\rho_{c} \bar{\rho}, \quad p=p_{c} \bar{p},
$$


where $l$ is the length of the field line, $v_{c}=l / t_{c}$ is the conductive velocity, $T_{c}=10^{6} \mathrm{~K}$. $\rho_{c}=8.35 \times 10^{-13} \mathrm{~kg} \mathrm{~m}^{-3}$ and $p_{c}=R \rho_{c} T_{c} / \tilde{\mu}$. Defining the timescale, $t_{c}$, as the conduction timescale

$$
t_{c}=\frac{\gamma p_{c} l^{2}}{\kappa_{0}(\gamma-1) T_{c}^{7 / 2}},
$$

we obtain,

$$
\begin{gathered}
\frac{\partial \bar{\rho}}{\partial \bar{t}}+\frac{\partial(\bar{\rho} \bar{v})}{\partial \bar{x}}=0 \\
\frac{\rho_{c} \bar{\rho} \gamma v_{c}^{2}}{c_{s}{ }^{2}}\left(\frac{\partial \bar{v}}{\partial \bar{t}}+\bar{v} \frac{\partial \bar{v}}{\partial \bar{x}}\right)+\frac{\partial \bar{p}}{\partial \bar{x}}=0 \\
\frac{1}{\gamma}\left(\frac{\partial \bar{p}}{\partial \bar{t}}+\bar{v} \frac{\partial \bar{p}}{\partial \bar{x}}\right)+\bar{p} \frac{\partial \bar{v}}{\partial \bar{x}}=\frac{\partial}{\partial \bar{x}}\left(\bar{T}^{5 / 2} \frac{\partial \bar{T}}{\partial \bar{x}}\right)-b\left[\bar{\chi}^{2} \bar{T}^{\alpha-2}-\bar{H}\right] \\
\bar{p}=\bar{\rho} \bar{T}
\end{gathered}
$$

where we have defined the sound speed squared as $c_{s}{ }^{2}=\gamma p_{c} / \rho_{c}$ and $H=H_{c} \bar{H}$ (where $H_{c}=\rho_{c}{ }^{2} \chi_{c} T_{c}{ }^{\alpha_{c}}$ and $\chi_{c}$ and $\alpha_{c}$ are the values of the temperature dependent parameters when $T=T_{c}$ ). We have also introduced

$$
\bar{\chi}=\frac{\chi T_{c}^{\alpha}}{\chi_{c} T_{c}^{\alpha_{c}}}
$$

for each particular range of temperature for the piecewise fit as well as

$$
b=\frac{\rho_{c}^{2} \chi_{c} T_{c}^{\alpha_{c}} l^{2}}{\kappa_{0} T_{c}^{7 / 2}},
$$

as the ratio of the conduction and radiative timescales.

If we consider the case where the conductive velocity $v_{c}$ is much smaller than the sound speed $c_{s}$, then equation (2.32) can be approximated by

$$
\frac{\partial \bar{p}}{\partial \bar{x}} \approx 0
$$

that is, $\bar{p}=$ constant $(=1)$ and we have an isobaric situation, that is, the radiative timescale is considerably longer than the acoustic timescale allowing any temperature and density variations to be in pressure equilibrium with their surroundings. We do not consider the solution $\bar{p}=\bar{p}(\bar{t})$ to (2.37). This has the computational convenience in that the numerical timesteps are not restricted by the need to resolve adequately the sound waves travelling back and forth across the system. Instead the slower thermal variations can be followed with timesteps only restricted by the physical processes that we wish to investigate. 
Thus the reduced set of isobaric equations is

$$
\begin{aligned}
\frac{\partial \rho}{\partial t}+\frac{\partial(\rho v)}{\partial x} & =0 \\
\frac{\partial v}{\partial x} & =\frac{\partial}{\partial x}\left(T^{5 / 2} \frac{\partial T}{\partial x}\right)-b\left[\chi T^{\alpha-2}-H\right] \\
\rho & =\frac{1}{T},
\end{aligned}
$$

where all bars have been removed for convenience.

These equations will be numerically solved for a typical coronal loop. The loop has a hot summit temperature $\left(\approx 1-2 \times 10^{6} \mathrm{~K}\right)$ and cool footpoints $\left(\approx 10^{4} \mathrm{~K}\right)$ embedded in the chromosphere. It is assumed that the loop is symmetrical and therefore it is only necessary to consider half the loop length, so that $0 \leq x \leq 0.5$ in the non-dimensionalised coordinates. Thus, equations (2.38) $-(2.40)$ are solved with the following boundary conditions,

$$
\begin{aligned}
\frac{\partial T}{\partial x} & =0, \quad \text { at } \quad x=0, \\
T & =T_{e}, \quad \text { at } \quad x=0.5,
\end{aligned}
$$

where $T_{e}$ is the chromospheric temperature and with the initial conditions at $t=0$ as,

$$
\begin{aligned}
v(x) & =0 \\
T & =T_{s}(x)
\end{aligned}
$$

where $T_{s}(x)$ is the static temperature profile obtained for a constant value of the heating $\left(H=H_{0}\right)$. 


\section{Chapter 3}

\section{Thermal Equilibria of Coronal Loops}

He was one of the most famous of mathematicians who were openly interested in "how the real world works". These scholars broke with the cloistered traditions of mathematics in several important ways. For one thing, they used computers constantly. For another, they worked almost exclusively with nonlinear equations. For a third, they appeared to care that their mathematics described something that actually existed in the real world. And finally, as if to emphasise their emergence from academia into the world, they dressed and spoke with what one senior mathematician called "a deplorable excess of personality". In fact, they often behaved like rock stars.

(Description of Ian Malcolm, Chaos Mathematician from 'Jurassic Park' by Michael Crichton)

\subsection{Introduction}

As mentioned in Chapter 1, the Sun's corona is not homogeneous and uniform but is a complex and dynamic environment consisting of a large variety of loop structures that are thought to outline the magnetic field. Observations from the Soft X-ray Telescope (SXT) on Yohkoh (Strong, 1994) show a range of loop sizes and shapes with active regions appearing as bright tangles of magnetic field lines surrounded by a network of large scale loops in the Sun's quiet regions. These recent observations indicate that active region loops are in constant motion, forming then reforming, and they appear to be slowly moving outwards from the Sun's surface, carrying 
plasma with them, the magnetic flux tubes confining the ionised plasma due to the high conductivity in the corona (see Figure 3.1).

Exotic loop geometries (twisted, sheared, kinked or cusped) appear possible with the magnetic structures showing changes on timescales that range from seconds to months. Loops also occur that stretch between active regions and quiet regions often over distances $>100 \mathrm{Mm}$. Table (3.1) gives physical parameter ranges for the different classes of loops seen in the corona (Steele, 1992).

\begin{tabular}{|c|c|c|c|c|c|}
\hline & $\begin{array}{c}\text { Inter- } \\
\text { connecting }\end{array}$ & $\begin{array}{c}\text { Quiet } \\
\text { Region }\end{array}$ & $\begin{array}{c}\text { Active } \\
\text { Region }\end{array}$ & $\begin{array}{c}\text { Post } \\
\text { Flare }\end{array}$ & $\begin{array}{c}\text { Simple } \\
\text { Flare }\end{array}$ \\
\hline Half Length $(\mathrm{Mm})$ & $5-17.5$ & $5-17.5$ & $2-25$ & $2-25$ & $1-10$ \\
Temperature $\left(10^{6} \mathrm{~K}\right)$ & $2-3$ & 1.8 & $0.01-2.5$ & $0.01-4$ & $<40$ \\
Particle Density & 7 & $2-10$ & $5-50$ & 100 & $<1000$ \\
$\left(10^{14} \mathrm{~m}^{-3}\right)$ & & & & & \\
Pressure $(\mathrm{Pa})$ & $0.02-0.03$ & 0.005 & $7 \times 10^{-5}$ & $0.01-5$ & 500 \\
& & -0.025 & -0.17 & & \\
\hline
\end{tabular}

Table 3.1: Physical parameters for different classes of magnetic loops found in the corona (Steele, 1992)

The thermal equilibrium temperature structure along a coronal loop has been modelled by many authors. Rosner et al (1978) derived a relationship for the dependence of the maximum temperature $\left(T_{\max }\right)$ along a loop of length $L$ with plasma pressure $p$ (gravity was neglected);

$$
T_{\max } \sim 1.4 \times 10^{3}(p L)^{1 / 3} .
$$

Hood and Priest (1979) solved the equations of thermal equilibrium in the absence of gravity and with a loop footpoint temperature set at $10^{6} \mathrm{~K}$, the transition region not included. They found that when the radiative term dominated the uniform heating along the loop, then, if the plasma pressure became too large or if the length of the fieldline considered was too long (by footpoint stretching or twisting motions), a lack of equilibrium occured and solutions with cool summit temperatures $<10^{5} \mathrm{~K}$ could exist.

Wragg and Priest (1981) extended the above work to include gravity. Loops with a constant pressure along their lengths were investigated and therefore the analysis was only relevent to loops smaller than the pressure scale height. They also considered a thermally isolated loop, that is, a loop for which the conductive flux 


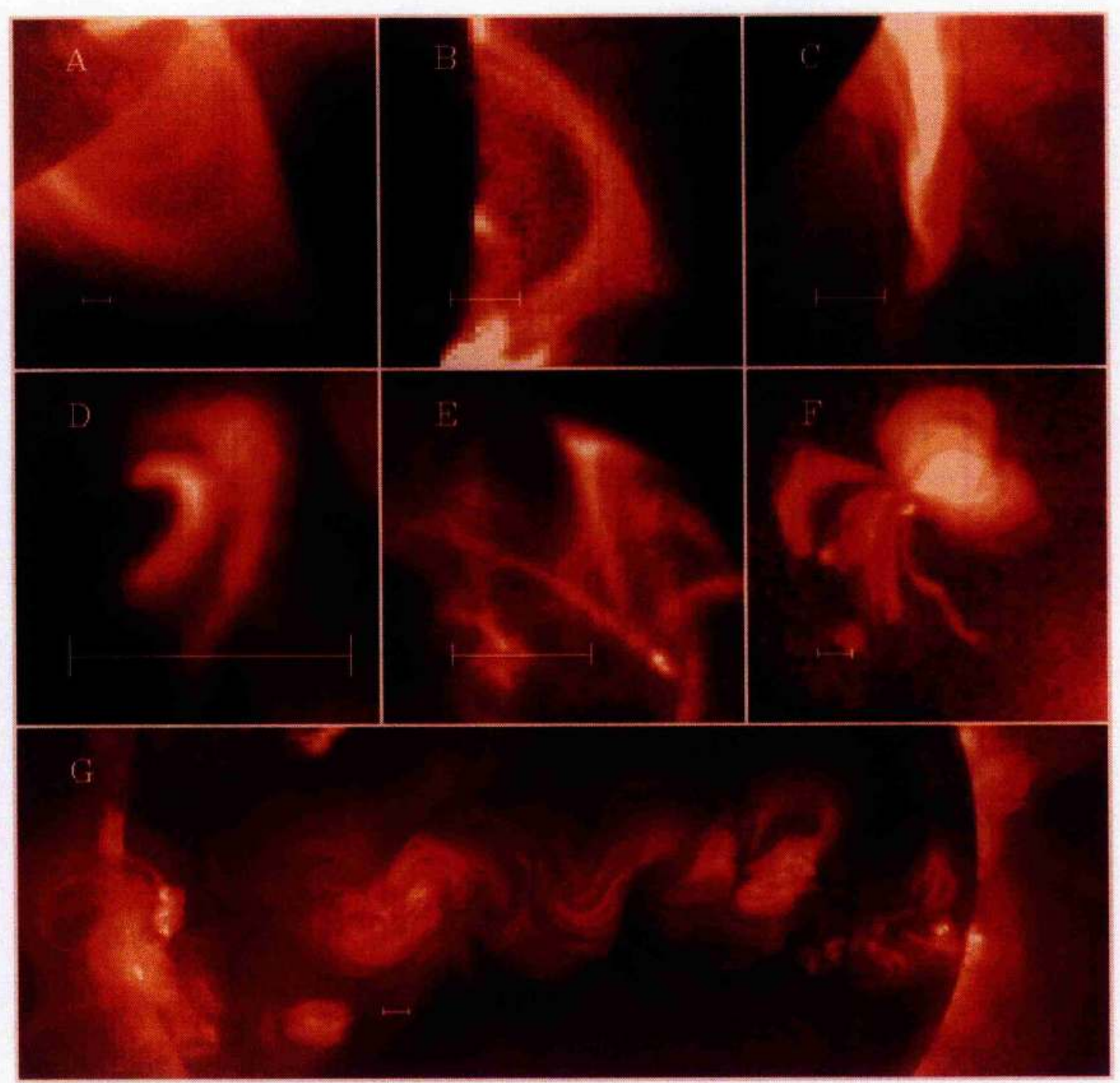

Figure 3.1: Images obtained from SXT on Yohkoh from $3^{\text {rd }}$ October 1991 to $25^{\text {th }}$ January 1992 showing a variety of coronal loop features; A: Large Helmet-type structure; B: Arcade of X-ray loops seen end-on; C: Dynamic eruptive loop growing at a velocity of $\sim 30 \mathrm{~km}^{-1}$; D: One of many small symmetrical flaring loops seen by SXT; E: Two cusped loops with heating in northern loop; F: Tightly beamed x-ray jet towards the southwest at $\sim 200 \mathrm{~km}^{-1}$; G: Section of solar disk showing loop structures in active regions. The horizontal line shows the relative size-scaling between the particular features. 
vanishes at both the loop summit and base, the base temperature set at a typical chromospheric value $\left(2 \times 10^{4} \mathrm{~K}\right)$.

She et al (1986) investigated the effect on the thermal equilibrium structure of placing the loop footpoints deep in the chromosphere, arguing that the corona could not simply be considered as a separate atmosphere from the transition region.

Observations of the differential emission measure (DEM) of the corona (which represents the amount of material present at a particular temperature) show a deep minimum at $10^{5} \mathrm{~K}$. The part of the DEM above $10^{5} \mathrm{~K}$ can be explained quite well by a static, hot loop model; however, the part below $10^{5} \mathrm{~K}$ cannot (that is, there is a greater amount of cooler material present than that simply calculated by using hot loops). The average DEM does not change very much across different solar regions and its.profile appears to be the same for all late-type stars.

Antiochos and Noci (1986) argued that if two different mechanisms were involved in producing this particular DEM profile then, for different areas of the Sun, the relative strengths of the two mechanisms should vary and thus the DEM would change - this is not the case. Thus, either the two mechanisms are strongly coupled or only one mechanism exists. Antiochos and Noci suggested that if hot coronal loop structures could explain the hot DEM part then the cool loop solutions of Hood and Priest (1979) could explain the cooler DEM part. Their model found that if both the hot and cool loops exist in the same region, the full form of the DEM could be reproduced. We will return to the DEM in Chapter 4.

Hood and Anzer (1988) quantitatively re-examined the equations of thermal equilibrium to find conditions under which cool condensations could form in the corona. They assumed a footpoint temperature of $2 \times 10^{4} \mathrm{~K}$ and a fixed base pressure, arguing that although a zero base conductive flux is attractive (since observations appear to show a " $2 \times 10^{4} \mathrm{~K}$-plateau" there), the actual physical mechanism which produces this plateau was not included in the model and therefore this effect should not be simulated. However, they did not allow the base conductive flux to get too large otherwise the chromospheric region would not be able to radiate away the strong conductive flux.

The authors used the powerful technique of phase plane analysis to investigate the thermal equilibrium solutions, altering the radiation and heating under certain conditions (no gravity, variation of cross-sectional area and gravity included respectively) so as to give an analytical phase plane solution. They argued that a general understanding of the overall physical effects was more important than specific details, provided that the changes made left that basic topology of the phase plane unchanged. 
Hood and Anzer argue against the cool loop of Hood and Priest (1979) explaining that their base temperature was too large $\left(10^{6} \mathrm{~K}\right)$ and that the cool solutions cannot be coupled onto the lower atmosphere as the base conductive flux is of the wrong sign. For a fuller explanation of the technique of phase plane analysis see Section 3.3 .

Steele and Priest (1990a) extended the work of Hood and Anzer (1988) by seeking numerical solutions to the thermal equilibrium equations for the Hildner (1978) radiative loss curve. Certain different types of thermal structure were identified. Hot loops had cool footpoints $\left(2 \times 10^{4} \mathrm{~K}\right)$ and hot summits $\left(4 \times 10^{5}-3 \times 10^{6} \mathrm{~K}\right)$. Cool loops had temperatures of $\sim 8 \times 10^{4} \mathrm{~K}$ along their entire length. Hot-Cool loops had footpoints at $2 \times 10^{4} \mathrm{~K}$ and a similar summit temperature or slightly lower but with intermediate portions at coronal temperatures. Warm loops are similar to cool loops but have summit temperatures between $8 \times 10^{4}-4 \times 10^{5} \mathrm{~K}$. They suggested that the global DEM could be explained by the hot-cool loop solution and that cool loops could have relevance for active region prominences where the magnetic field lies mainly along the plane of the prominence.

Steele and Priest (1991b) continued the above analysis to include changes in the cross-sectional area of the coronal loop. Their results showed that if the area of the flux tube was increased towards the summit, the summit temperature of (a) the hot and cool loops increased slightly, (b) the warm solutions were unchanged, and (c) the hot-cool loops increased dramatically.

Steele and Priest (1994) went on to include gravity which meant that the pressure along the loop fell from the footpoint to the summit. Since the pressure was now a free parameter, a phase volume had to be constructed by stacking many phase planes on top of one another. The most dramatic result of the effect of gravity was that the hot-cool loop no longer existed and therefore the number of solutions were reduced.

Steele and Priest (1990b, 1991a) also considered the thermal equilibria of a coronal magnetic arcade which they modelled as a continuum of individual fieldlines with their axis on or below the photosphere (see Figure 3.2). The thermal equilibrium structure of the arcade is then calculated by solving the thermal structure along each individual flux tube. This procedure is valid provided that the thermal conductivity along the field lines is much greater than across the field, allowing the loops to be thermally isolated from each other.

Hot loops (Alissandrakis et al, 1988) and cool loops (Foukal, 1981) have been observed in the corona and as well as the equilibria investigations, many authors have studied the stability of these loops (Antiochos, 1979; Hood and Priest, 1980; 


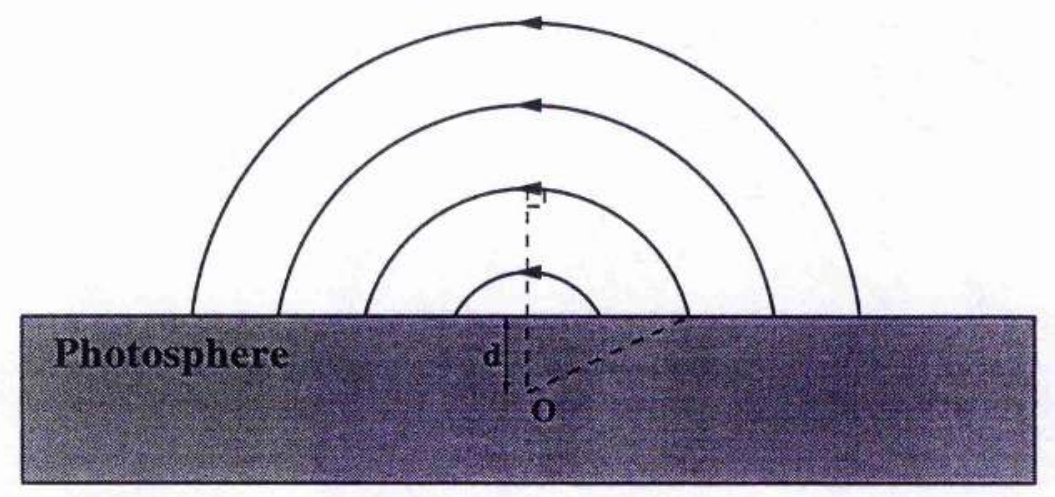

Figure 3.2: Form of the magnetic arcade as considered in Steele and Priest (1991a). It is made up of a continuum of fieldlines with their axis $(\mathrm{O})$ a distance $d$ below the surface of the photosphere.

Cally and Robb, 1995). In particular, Cally and Robb (1995) found that with gravity included, their cool loop solutions were unstable and evolved to hot loops in only a matter of minutes.

\subsection{The Thermal Equilibrium Equation and a Simplified Radiative Loss Function}

The equation of thermal equilibrium is obtained from equations (2.38) - (2.40) by setting all time derivatives to zero and by assuming that the velocity is identically zero. We are left with a balance between conduction, radiation and heating;

$$
\frac{d}{d x}\left(T^{5 / 2} \frac{d T}{d x}\right)=b\left[\chi T^{\alpha-2}-H_{0}\right],
$$

where $H_{0}$ is a constant value along the coronal loop. The boundary conditions are (2.41) and (2.42).

A proper description of the possible static solutions to the model equations is essential to understanding the overall system we are investigating. This is a complicated problem particularly when considering the continuous radiative loss functions shown in Figure 2.1. However, by choosing a particular simplified form of this function, it is possible to derive an analytical solution for these equilibrium states which will provide insight into the nature of the more complex problem. One simplified form is the Two Range Radiative Loss Function,

$$
\chi T^{\alpha-2}= \begin{cases}T^{-7 / 4}, & T>T_{r}, \\ \frac{T^{7 / 2}}{T_{r}^{21 / 4}}, & T<T_{r}\end{cases}
$$




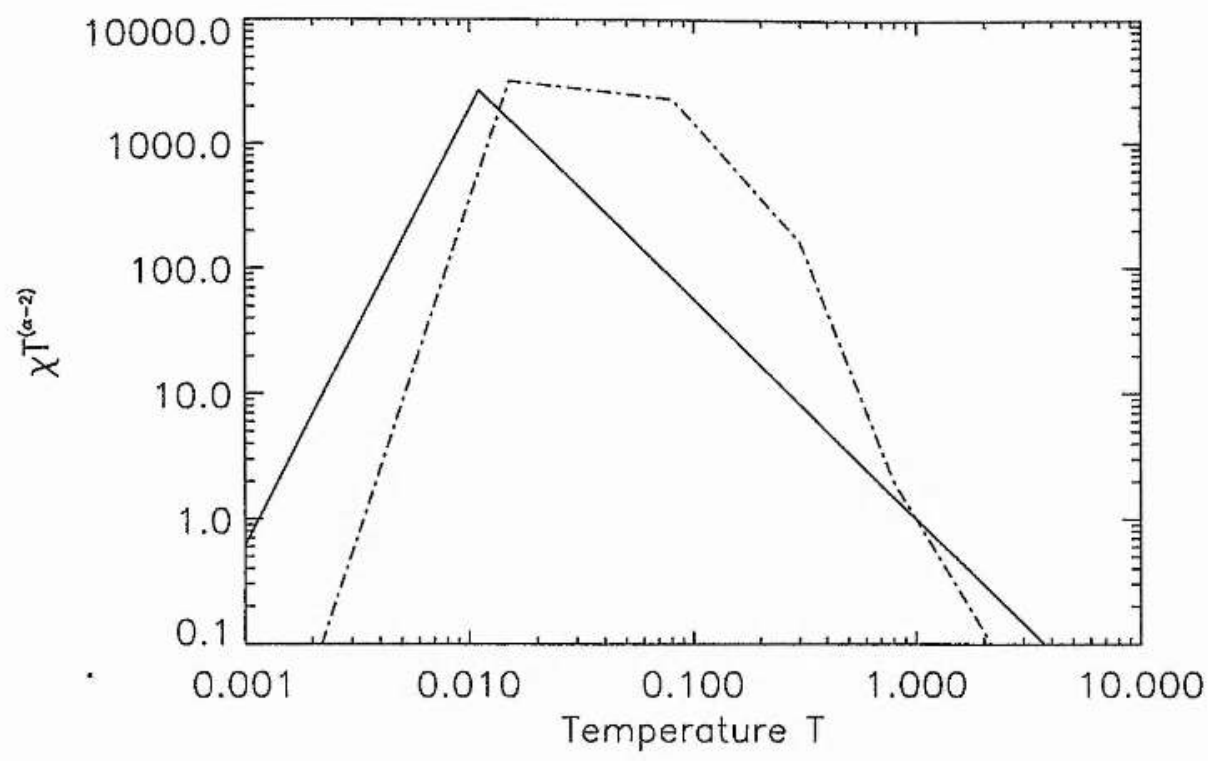

Figure 3.3: Comparison of (-) Two range Radiative Loss Function and (.-..) Hildner. 1974 in the non-dimensionalised form with $T_{r}=0.011$.

where $T_{r}$ is the temperature at the maximum of the radiative loss. What is important is that the simplified form should have the same properties as the calculated radiation functions. In this case for constant pressure, the radiation increases with decreasing temperature from coronal temperatures $\left(\approx 2 \times 10^{6} \mathrm{~K}\right)$ but eventually this situation reverses at $T_{r}$ and the radiation falls as the temperatures reach chromospheric values $\left(10^{4} \mathrm{~K}\right) . T_{r}$ is just above $10^{4} \mathrm{~K}$, remembering that the optically thin radiation is calculated at constant pressure and not constant density.

\begin{tabular}{|c|c|c|}
\hline$T$ & $\alpha$ & $\chi$ \\
\hline \hline$T<0.015$ & 7.4 & $2.24 \times 10^{13}$ \\
$0.015 \leq T<0.08$ & 1.8 & $1.37 \times 10^{3}$ \\
$0.08 \leq T<0.3$ & 0.0 & $1.45 \times 10^{1}$ \\
$0.3 \leq T<0.8$ & -2.5 & $7.15 \times 10^{-1}$ \\
$0.8 \leq T<10$ & -1.0 & 1 \\
\hline
\end{tabular}

Table 3.2: $\alpha$ and $\chi$ values (in non-dimensionalised form) for Optically Thin Radiation, obtained from analytical fit by Hildner (1974)

Figure 3.3 compares the simplified function for $T_{r}=0.011$ (in units of $10^{6} \mathrm{~K}$ ) with Hildner's estimates (in non-dimensionalised form) for the optically thin radiation (see Table 3.2). This approximation can only be considered down to chromospheric 
temperatures $\left(\sim 10^{4} \mathrm{~K}\right)$ - it will not be taken down to the temperature minimum region where radiation and heating balance as optically thick effects become important. This approximation to the radiative curve follows the philosophy of Hood and Anzer (1988) who deduced the properties of static loops by introducing simplified loss functions (see Section 3.1).

For ease of mathematical analysis, the equilibrium equation (3.2) can be written as

$$
\frac{d^{2} y}{d x^{2}}=\frac{7 b}{2}\left[\chi y^{2(\alpha-2) / 7}-H_{0}\right]
$$

where $y=T^{7 / 2}$. The boundary conditions (2.41) and (2.42) are now given as

$$
\begin{array}{ll}
\frac{d y}{d x}=0 & \text { at } \quad x=0 \\
y=y_{e} & \text { at } \quad x=0.5
\end{array}
$$

and where $y_{r}=T_{r}{ }^{7 / 2}$ for the simplified radiative function.

\subsection{Phase Plane Analysis}

As in Hood and Anzer (1988) and Steele and Priest (1990a), a lot of information about the nature of the solutions to equation (3.4) can be found by investigating the corresponding phase plane diagram where the conductive flux is given as a function of temperature. Setting,

$$
\begin{gathered}
\frac{d y}{d x}=\Phi, \\
\frac{d \Phi}{d x}=\frac{7 b}{2}\left[\chi y^{2(\alpha-2) / 7}-H_{0}\right],
\end{gathered}
$$

then the critical points in the $(y, \Phi)$ space occur when these two derivatives are zero, that is,

$$
y=y_{\text {crit }}=\left(\frac{H_{0}}{\chi}\right)^{7 / 2(\alpha-2)}, \quad \Phi=0 .
$$

It must be remembered that the values of $\chi$ and $\alpha$ are different on either side of $y=y_{r}$ for the Two Range Loss Function. If $H_{0}<y_{r}{ }^{-1 / 2}$ then two critical points exist;

$$
y_{\text {crit }}=y_{h}=H_{0}^{-2}
$$

for $\chi=1$ and $\alpha=1 / 4$ in the upper, hotter region and

$$
y_{\text {crit }}=y_{c}=H_{0} y_{r}^{3 / 2}
$$


for $\chi=y_{r}{ }^{-3 / 2}$ and $\alpha=11 / 2$ in the lower, cooler region of the radiation curve. A critical point analysis (Bender and Orzag, 1978) gives $\left(y_{h}, 0\right)$ as a centre point and $\left(y_{c}, 0\right)$ as a saddle point (see Appendix A).

Figure 3.4 shows the phase space for $H_{0}<y_{r}{ }^{-1 / 2}$ in terms of temperature $T$ and the conductive flux $T^{5 / 2} d T / d x$. The values for $H_{0}(=3.2)$ and $b(=20)$ are purely arbitrary. Consider a footpoint temperature $T_{e}$ that lies along the line $L_{1}(-.-$. where $T_{c}<T_{e}<T_{h}$. Starting at point $A_{1}$ and noting that the direction of rotation around the closed contours is clockwise, the contour $A_{1} A_{2}$ gives a thermally isolated loop with a hot summit. It is thermally isolated as the base conductive flux is zero and the summit temperature of the loop is the minimum value for a hot loop solution with this particular footpoint temperature.

There is the possibility of continuing the contour round back to $A_{1}$ again (that is, $\left.A_{1} A_{2} A_{1}\right)$ to give a cool summit temperature. Such a loop is hot along most of its length apart from the cool summit and footpoint and is therefore called the hot-cool solution. We will not be considering this type of loop.

Beginning at point $B_{1}$, a hot solution occurs with summit temperature $B_{2}$ and similarly for $C_{1} C_{2}$ (which lie on the separatrix curve). $D_{1} D_{2}$ also is a hot solution but now, since the contour is no longer closed, a hot-cool solution is no longer possible.

If the base conductive fluxes are negative, then cool solutions can occur (for example, $B_{3} B_{4}$ ). As the base conductive flux approaches $C_{3}$ (which lies on the separatrix curve), the loops lengthen considerably as the contour passes very close to $T_{c}$. Thus long, nearly isothermal cool solutions exist. It must be noted that these cool solutions are normally rejected as unphysical since the summit temperature is lower than the transition region footpoint temperature and that the conductive flux at the base is of the wrong sign. For conductive fluxes less than $C_{3}$ (such as $\left.D_{3}\right)$ ), no cool solutions are possible.

Now consider a footpoint temperature $T_{c}>T_{e}$ along the line $\dot{L}_{2}$. Starting with the footpoint at point $E_{1}$ gives a completely cool solution along the loop length with summit temperature at $E_{2}$. The temperature of the loop never rises to coronal values and is the cool solution referred to by Antiochos and Noci (1986).

The contour $F_{1} T_{c}$ also represents a cool solution but since $T_{c}$ is a critical point, it takes an infinite distance to integrate up to that particular temperature - thus the loop is infinitely long and is basically isothermal at temperature $T_{c}$.

If the base conductive flux is increased to $G_{1}$ so that the contour lies outside the separatrix curve then only a hotter solution is possible $\left(G_{1} D_{1} D_{2}\right)$. It can be argued that two solutions can occur. The length of the loop can be increased (a) by increasing the base conductive flux or (b) by decreasing the base conductive flux 


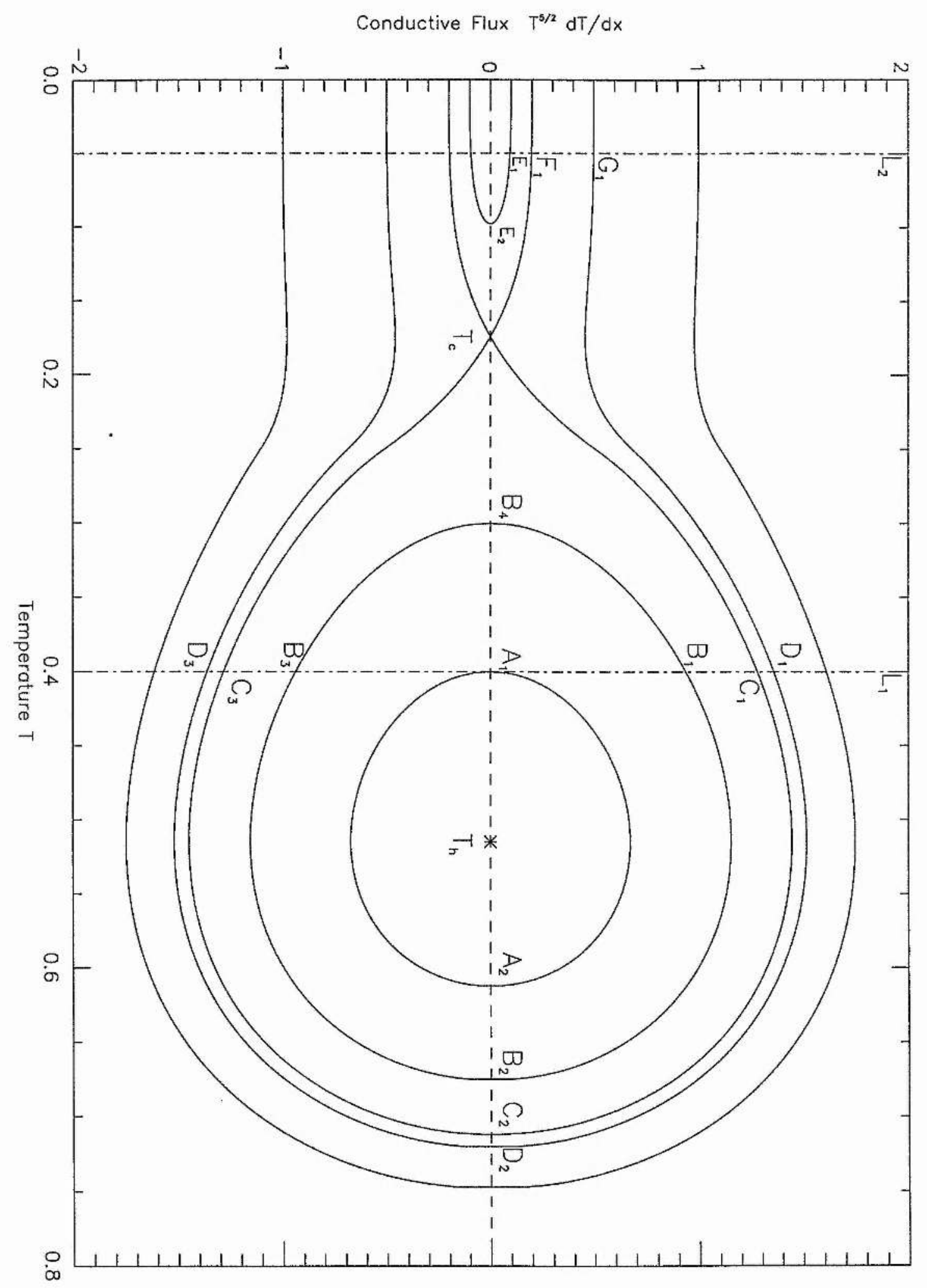

Figure 3.4: Form of phase plane solution for $H_{0}<y_{r}{ }^{-1 / 2}$ with $H_{0}=3.2$ and $b=20$ for $T_{r}=0.25$. 


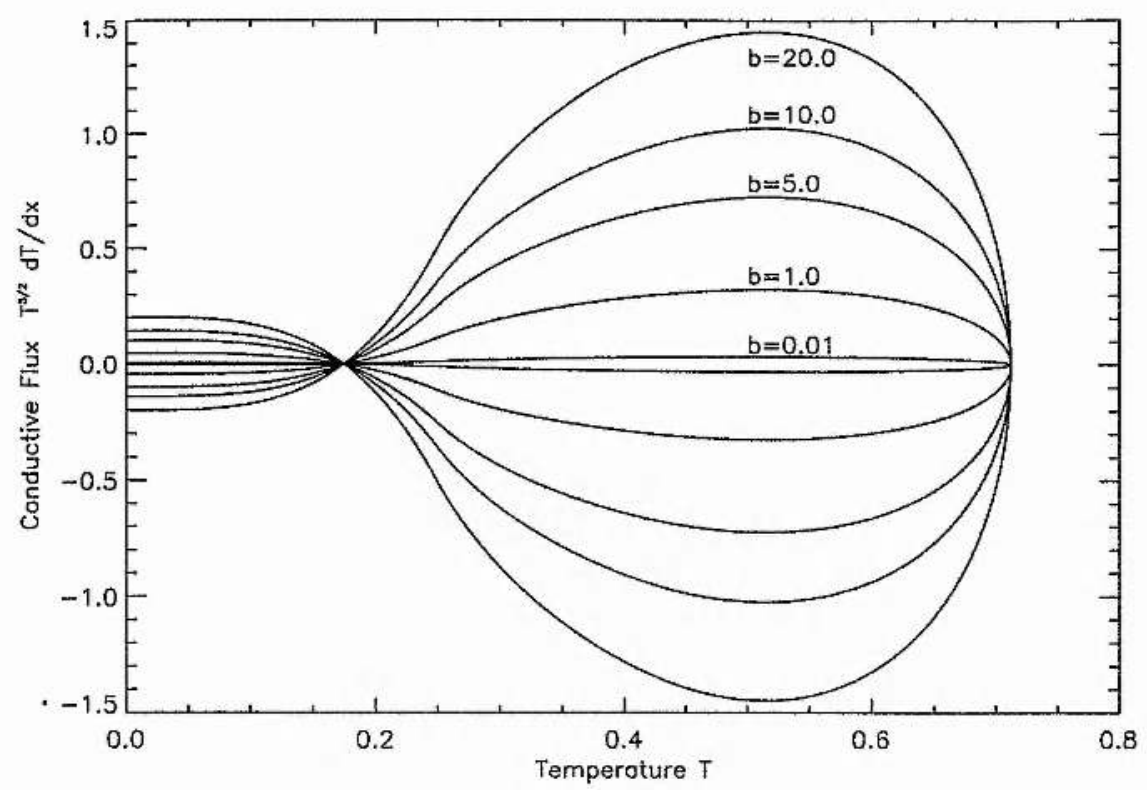

Figure 3.5: Form of phase plane separatrix curve for $H_{0}=3.2$ and $T_{r}=0.25$ with increasing values of $b$.

towards $F_{1}$ but still remaining outside the separatrix curve. In the case (a), the loop summit temperature increases and therefore a hot solution exists. For case (b), the contour passes very close to $T_{c}$ and therefore will spend a long time in the neighbourhood of this point, the loop summit temperature being very close to the value at $C_{2}$. This has been termed the warm solution and thus, there is a minimum summit temperature of warm loops as defined by the separatrix curve.

Figure 3.5 shows how the phase plane diagram changes with increasing values of $b$. The expanding contours scale as $b^{1 / 2}$. Figure 3.6 shows the phase plane solutions for $H_{0}>y_{r}{ }^{-1 / 2}$ - for footpoint temperatures along $L_{3}$ only hot solutions exist $\left(Z_{1} Z_{2}\right.$ for $H_{0}=20, W_{1} W_{2}$ for $H_{0}=50$ and $U_{1} U_{2}$ for $H_{0}=100$ ).

\subsection{Analytical Solution for the Dependence of the Loop Summit Temperature upon Loop Length}

The Two Range Radiative Loss Function was chosen so that an analytical solution to the thermal equilibria could be derived. Thus, it is possible to obtain an analytical expression for the dependence of the summit temperature $T_{0}$ upon the length of the fieldline (through $b$ ) for different values of $H_{0}$. There are two possibilities; 


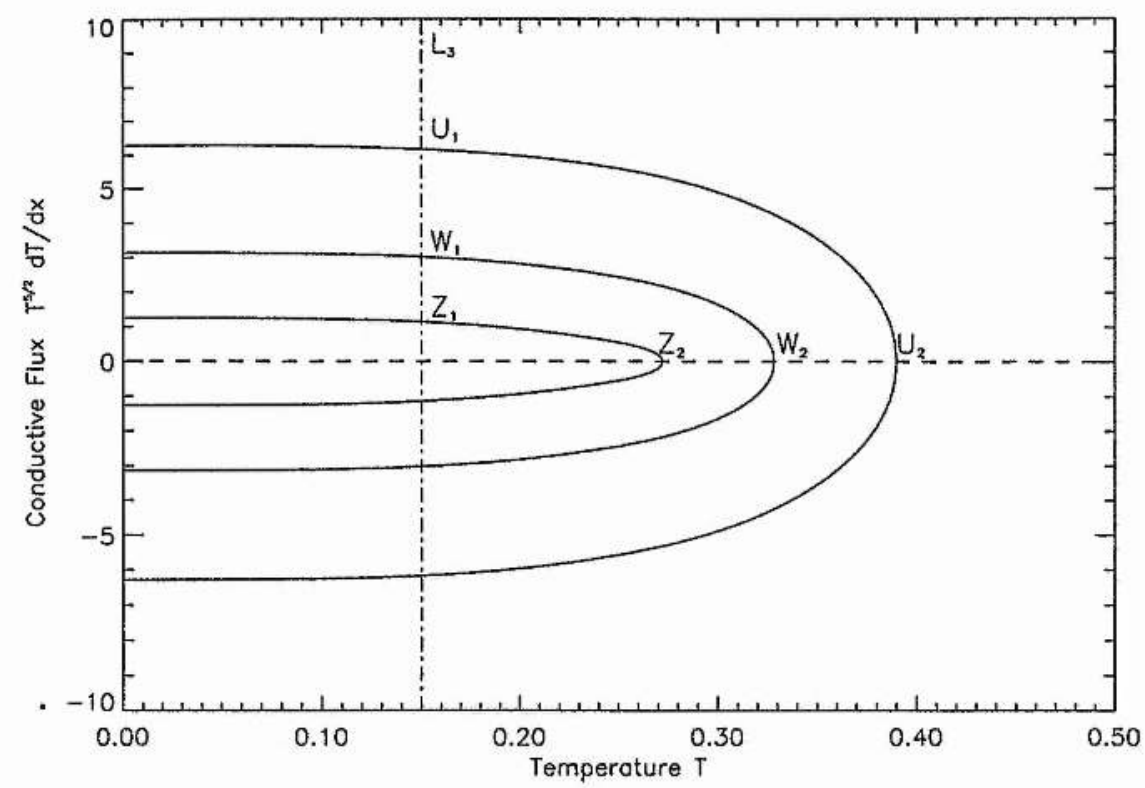

Figure 3.6: Form of phase plane solution for increasing values of $H_{0}(20,50,100)>y_{r}{ }^{-1 / 2}$ with $b=20$ and $T_{r}=0.25$.

(i) $T_{0}>T_{r}>T_{e}$;

$$
\begin{aligned}
\frac{7 b}{2}=[ & {\left[2 \sqrt{\frac{2}{H_{0}^{3}}}\left\{\cos ^{-1}\left(\frac{1-H_{0} y_{r}^{1 / 2}}{H_{0} y_{0}^{1 / 2}-1}\right)+\frac{\sqrt{2 H_{0}}}{y_{r}{ }^{3 / 4}} C_{1}\right\}\right.} \\
& \left.+2 y_{r}^{3 / 4} \ln \left(\frac{C_{2}-\sqrt{C_{2}{ }^{2}-4\left(C_{3}{ }^{2}-C_{1}^{2}\right)}}{2\left(C_{3}-C_{1}\right)}\right)\right]^{2}
\end{aligned}
$$

where

$$
\begin{aligned}
& y(0)=y_{0}=T_{0}^{7 / 2}, \quad y_{r}=T_{r}^{7 / 2}, \quad y_{e}=T_{e}^{7 / 2}, \\
& C_{1}= \frac{y_{r}^{3 / 4}}{\sqrt{2 H_{0}}}\left\{\left(1-H_{0} y_{0}^{1 / 2}\right)^{2}-\left(1-H_{0} y_{r}^{1 / 2}\right)^{2}\right\}^{1 / 2}, \\
& C_{2}=y_{e}-H_{0} y_{r}^{3 / 2}, \\
& C_{3}=\frac{1}{2}\left(y_{r}-H_{0} y_{r}^{3 / 2}\right)
\end{aligned}
$$

(ii) $T_{0}, T_{e}<T_{r}$;

$$
\frac{7 b}{2}=4 y_{r}^{3 / 2}\left[\cosh ^{-1}\left(\frac{y_{e}-H_{0} y_{r}^{3 / 2}}{y_{0}-H_{0} y_{r}{ }^{3 / 2}}\right)\right]^{2} .
$$

The derivation of these expressions can be found in Appendix B. Both cases are shown in Figure 3.7 for $T_{e}=0.01$ and $T_{r}=0.011$ in units of $10^{6} \mathrm{~K}$. The curves defining the dependence of $T_{0}$ upon $b$ have two different forms depending on the 


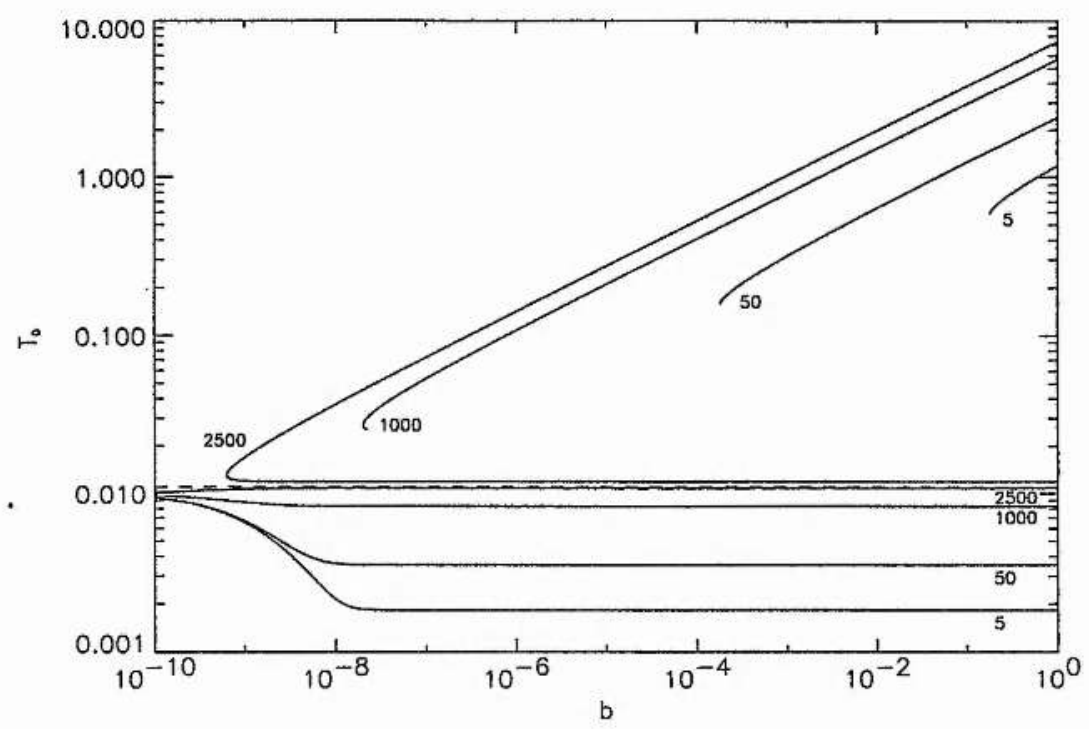

Figure 3.7: Dependence of the summit temperature $T_{0}$ (in units of $10^{6} \mathrm{~K}$ ) upon parameter $b$ for different values of the constant heating term $H_{0} . T_{0}=T_{r}$ is the dashed line.

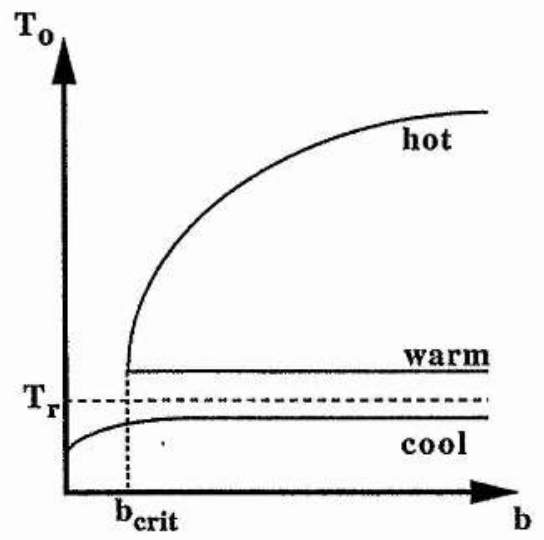

(A)

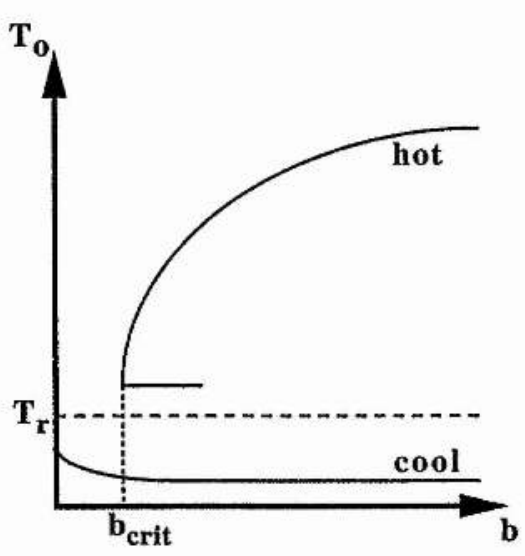

(B)

Figure 3.8: Two cases for the dependence of the summit temperature upon parameter $b$ for (A) $T_{c}>T_{e}$ and (B) $T_{c}<T_{e}$. 


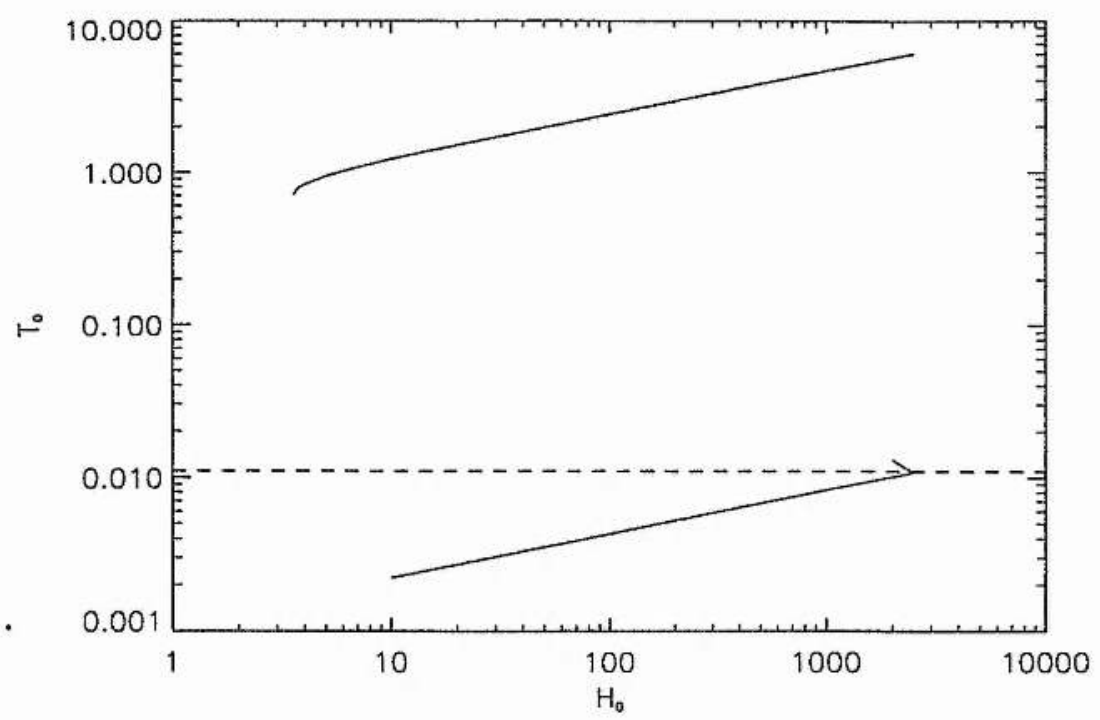

Figure 3.9: Variation of summit temperature $T_{0}$ (in units of $10^{6} \mathrm{~K}$ ) with $H_{0}$ for $b=0.5$ and $T_{e}=0.01$ (i.e. $\left.10^{4} \mathrm{~K}\right)$.

value of $H_{0}$. Given that $T_{c}=H_{0}{ }^{2 / 7} T_{r}^{3 / 2}$ is the value of the saddle point temperature, the form of the curve in Figure 3.7 depends upon whether $T_{c}$ is less than or greater than $T_{e}$. Figure 3.8 show these two cases.

For case (A), $T_{c}>T_{e}$ and three solutions exist (hot, warm and cool) as described in Section 3.3. For $b$ less than some critical value $\left(b_{\text {crit }}\right)$, only cool solutions exist. It can be shown that the hot and cool solutions are linearly stable whereas the warm solution is not. Thus, temperatures above the hot solution will evolve towards it. However, if the temperature drops below the warm value, the solution will be attracted to the lower curve creating a cold plasma along the loop length.

For case (B), $T_{c}<T_{e}$ and a warm solution no longer exists. If the summit temperature falls too far away from the hot solution, it will continue to fall towards the cool solution. The change over between these two cases occurs for $H_{0}=y_{e} y_{r}{ }^{-3 / 2}$ $(\approx 1917$ for the above parameters).

Even if the heating is subsequently increased, it is difficult for the plasma to move away from the cool solution once it has been reached. This is illustrated in Figure 3.9 where $T_{0}$ is plotted against $H_{0}$ for a fixed value of parameter $b(=0.5$ in this case) and where $T_{e}=0.01$ in the non-dimensionalised units - we see that a large amount of heat $\left(H_{0}>y_{r}{ }^{-1 / 2} \approx 10^{3.43}\right.$ in this case) would be required to retrieve a hot loop solution, this value of the heating corresponding to Figure 3.6 when only hot solutions exist. 


\subsection{Conclusion}

In this chapter a detailed investigation of the possible static solutions of the thermal equilibrium along a coronal loop was carried out. Due to the form of the radiative loss curve in the corona, this is generally a complicated problem. However, a simplified form of the radiation, which has all the same properties of the full problem, was introduced. This allowed for the analytical investigation of the equilibrium solutions by using the powerful technique of phase plane analysis. Hot, cool, warm and hot-cool solutions are possible, depending on the length of the loop, the footpoint temperature, the base conductive flux and the value of the heating term.

The effect on the thermal structure of varying the heat source in time will be investigated in the next chapter. 


\section{Chapter 4}

\section{Time-Dependent Solar Coronal Heating}

"The Sun has always kept its secrets well"

(J. Gribbin, 'Blinded by the Light - the Secret life of the Sun')

\subsection{Introduction}

Satellite and rocket telescope observations have revealed that the Sun exhibits a wide variety of dynamic phenomena. As discussed in Chapter 1, there are many examples of the interaction of the coronal plasma with the Sun's coronal magnetic field. Up until about 1940, it was thought that the temperature of the Sun decreased from the solar surface. However, one of the most perplexing problems in solar physics is to explain the fact that while the photosphere (which emits most of the visible solar radiation) is at $6000 \mathrm{~K}$, the temperature passes through a minimum at $\sim 4300 \mathrm{~K}$ and then begins to rise through the chromosphere $\left(10^{4} \mathrm{~K}\right)$ and transition region $\left(10^{5} \mathrm{~K}\right)$, before reaching between $\sim 2 \times 10^{6} \mathrm{~K}$ in the corona. Since the 1970 's, this particular feature has been known to occur in other solar-like stars. Chromospheric heating can be explained in terms of the dissipation of magnetoacoustic waves (see Narain and Ulmschneider, 1990, 1995 and references therein) but the nature of the mechanism in the solar corona is less certain.

Parker (1987a) states that as the solar corona is in an extreme state of dynamical motion, the whole atmosphere would collapse in only one hour if the heating source was suddenly switched off! So the corona is maintained and sustained hour by hour by its unknown heating supply. Any coronal heating model should be able to explain

- the energy losses from both quiet and active regions; 
- the variation of the corona's temperature with the solar cycle;

- the heating of coronal loop structures, formation of X-ray bright points and the presence of coronal holes.

Due to refraction effects at the corona-photospheric boundary, coronal heating by acoustic waves has generally been regarded as negligible (Anderson and Athay, 1989a,b). However this view has been challanged by Dere and Mason (1993) who studied the non-thermal velocities for spectral lines primarily covering the temperature range $10^{4}$ to $2 \times 10^{6} \mathrm{~K}$ and found that they were significantly higher than those suggested in previous studies. Dere and Mason conclude that the acoustic wave flux inferred from these non-thermal line broadenings would be sufficient to supply the energy to balance losses for most regions of the solar atmosphere.

In spite of this, it has generally been accepted that the Sun's magnetic field plays the most important part in understanding the coronal heating problem. Observational data of specific heating mechanisms has been difficult to obtain due to a number of reasons (Zirker, 1993):

- Several heating mechanisms may be operating at once.

- Heating release events may be too small to be spatially resolved by present instrumentation. This is a direct consequence of the fact that the coronal plasma is very inefficient at dissipation and therefore small scales are required for effective energy release.

- If the heating is in discrete events (which is suggested by one of the mechanisms), the frequency of these events may be too high to be adequately temporally resolved.

- Any unique signatures of specific methods may be destroyed during the thermal release of the injected energy.

Attempts have been made to detect indirectly the heating mechanism (see Cargill, 1994a for a summary of possible diagnostic tools) and observational evidence for each coronal heating theory is presented below in each relevant section.

Note that it is not the purpose of this chapter to provide a detailed review of the physics of coronal heating methods (see Priest, 1993 for an excellent overview of the various theories). Rather the aim of this part of the thesis is to understand the response of the coronal plasma to a time-varying supply of heat. A time-dependent heating term is required because none of the present heating mechanisms are likely to provide a constant, continual supply of heat energy. 
In Section 4.2 there is a description of a number of heating theories suggested from literature. The time dependent heating term to be investigated is introduced in Section 4.3 and a test for the numerical code is considered in Section 4.4 using an initially isothermal atmosphere. This has the advantage that it is possible to analytically derive a formula for the thermal evolution of the loop (see Appendix C). The results of time dependent heating of a typical coronal loop structure are shown in Sections 4.5 and 4.6 while the effect upon the differential emission measure is described in Section 4.7. The results in this chapter are summarized in Walsh et al $(1995 \mathrm{a}, \mathrm{b})$.

\subsection{Heating Mechanisms in the Corona}

Various heating methods have been suggested in order to balance the energy losses from the corona by radiation, thermal conduction and plasma mass flow (Withbroe and Noyes, 1977 - see Table 4.1 for estimated values for quiet regions, active regions and coronal holes). Cargill (1994a) points out that these estimates are nearly twenty years old and are taken from a region of the spectrum that has not been investigated in any detail since the 1970's. SOHO will be carrying a wide range of EUV instruments and it is very important that the Withbroe and Noyes values are confirmed or updated as necessary.

\begin{tabular}{|c|c|c|c|}
\hline $\begin{array}{c}\text { Coronal Fluxes } \\
\text { ergs }^{-1} \mathrm{~cm}^{-3}\end{array}$ & Quiet Sun & Coronal Hole & Active Region \\
\hline \hline Conduction & $2 \times 10^{5}$ & $6 \times 10^{4}$ & $10^{5}-10^{7}$ \\
Radiation & $10^{5}$ & $10^{4}$ & $5 \times 10^{6}$ \\
Solar Wind & $\leq 5 \times 10^{4}$ & $7 \times 10^{5}$ & $<10^{5}$ \\
Total & $3 \times 10^{5}$ & $8 \times 10^{5}$ & $10^{7}$ \\
\hline
\end{tabular}

Table 4.1: Estimated values for energy losses on the Sun as calculated by Withbroe and Noyes, 1977.

\subsubsection{Wave Heating of the Corona}

Given the fact that acoustic waves appear to be unable to balance coronal losses, other forms of wave heating have been investigated. A likely candidate, Magnetoacoustic Waves, has generally been dismissed in the literature. However, this has been recently contested by Porter et al $(1994 \mathrm{a}, \mathrm{b})$. They argue that the slow 
mode fluxes derived from Doppler line shift measurements in the chromosphere and transition region may have been underestimated by two orders of magnitude due to the lack of resolution of small scale motions. The real slow mode energy fluxes might well be sufficient to match the coronal losses from a quiet region. Porter et al also suggest that another possible source of the waves could be from an "impulsive, bursty regime" generated by a magnetic reconnection event (see Forbes and Priest, 1982).

Many authors have concentrated their efforts towards the generation, propagation and dissipation of Alfvén Waves in the corona. The ultimate source of energy lies in the convective motions in and below the photosphere and therefore wave heating by Alfvén waves has two problems to overcome;

1. They are difficult to excite by the convective motions;

2. They are difficult to damp in the corona, partly because of their incompressible nature.

\section{Resonant Absorption}

One possible mechanism for overcoming these problems is Resonant Absorption. This requires a wave with a frequency within either the Alfvén or slow continuum to propagate in from the photospheric boundary. When the frequency of the incoming wave matches the local frequency of a continuum mode, the fieldline resonates and a large amplitude develops. Non-ideal MHD dissipation limits the growth of the resonant mode and dissipates the incoming wave energy into heat.

It was in the context of thermonuclear fusion research that resonant absorption of Alfvén waves was first considered as a viable plasma heating mechanism. For fusion to occur, it is necessary to confine a sufficiently hot and dense plasma for a long enough time so that the power necessary to heat the plasma is exceeded by the power produced by the fusion reactions themselves. The most promising way of achieving this is by using a machine called a tokamak (Wesson, 1987). It has an axisymmetric toroidal configuration with strong toroidal and weaker poloidal magnetic fields. The plasma itself generates a poloidal field which confines the current and provides Ohmic heating of the plasma. However, as the temperature rises, the plasma resistance falls off as $T_{e}^{-3 / 2}$, where $T_{e}$ is the electron temperature. Therefore, an extra source of heating is required to push the plasma into the ignition regime. Resonant absorption was investigated as a means of adding the extra energy required (for example, Poedts, Kerner and Goossens, 1989).

Ionson (1978) was one of the first to consider resonant absorption as a possible 
source of coronal loop heating; he concluded that an Alfvénic surface wave could, when dissipated, irreversibly heat the supporting, nonuniform plasma, the energy deposition being very localised in a thin sheath $(\approx 1 \mathrm{~km}$ wide) which would envelope the entire length of the loop.

Hollweg (1984) showed how a coronal loop could act as a resonant cavity and that the transmission of energy into the loop was enhanced at certain resonant frequencies. His results were in qualitative agreement with Ionson's results. Once excited, Alfvén waves do not dissipate readily; for example, in order to achieve the necessary heating rate for the quiet corona, a $100 \mathrm{~s}$ period wave would be required to damp in only a few journeys along a typical coronal loop. Possible dissipative mechanisms such as viscosity, radiative damping and resistivity require steep gradients to be effective. Hollweg (1984) mentions turbulence as a means of transferring the energy from large to small scales where dissipation can take effect due to the large velocity and magnetic gradients. For a fuller discussion of MHD turbulence see Section 4.2.2.

Davila (1987) produced very convincing results for the resonant absorption process, where an Alfvén wave becomes trapped within a coronal loop, "bouncing back and forth" between the loop ends. If the driving frequency of the system is just right then the captured energy will increase and eventually be dissipated within the loop.

The numerical simulations of Poedts, Goossens and Kerner (1989), in which they investigate the efficiency of resonant absorption in the framework of linearized, compressible, resistive MHD, seem to confirm Davila's findings. They state that as ideal MHD is conservative, it alone is not a good modeller of the resonant absorption process as the energy absorbed by the plasma can never be dissipated but rather accumulates in an ever diminishing region around the resonant layer.

Poedts, Belien and Goedbloed (1994) extended this idea to define the quality of a resonance, $Q$, as the ratio of the total energy contained in the systern to the dissipation per driving cycle of the external source (that is, the incident waves) and the fractional absorption, $f_{a}$, of the heating process as a measure for the coupling of the external driving source to the coronal loop plasma. Good plasma driver coupling does not necessarily mean efficient plasma heating. However, they found that the resonances in coronal loops have bad quality - the dissipation per cycle is appreciable, allowing them to conclude that resonant absorption is a possible heating mechanism.

Ruderman and Goossens (1993) studied the resonant absorption of small amplitude surface Alfvén waves in non-linear incompressible MHD for a viscous and resistive plasma. They found that the introduction of non-linearity caused an addi- 
tional increase in the wave damping rate with its value far exceeding that predicted by linear theory.

Ofman and Davila (1994) were the first to study the non-linear aspects of resonant absorption of Alfvén waves in three dimensions, arguing that non-linear effects may be important in regions of large velocity shear. They found that the location of the Alfvén resonant surfaces are time dependent as was the ohmic heating rate which saturated when the dissipation balanced the periodic energy input from their photospheric driver.

Wright and Rickard (1995) investigated the response of a non-uniform, onedimensional MHD cavity that was driven by a prescibed random boundary motion with a broad-band frequency spectrum. Their numerical results showed that the resonant excitation of Alfvén waves can still occur when a cavity is driven by a non-monochromatic source. They applied their results to laboratory plasmas and the magnetospheric cavity bounded by the magnetopause as well as coronal loops.

Erdelyi and Goossens (1995) extended the numerical code developed by Poedts, Goossens and Kerner (1989) to include viscous as well as resistive terms. Under solar conditions, both dissipative terms are operating with the electrical resistivity being slightly more important.

\section{Phase-Mixing}

In 1983, Heyvaerts and Priest introduced the idea of coronal heating by the phasemixing of sheared Alfvén waves. The photospheric footpoints of a coronal loop are oscillated at a certain fixed frequency. Since each fieldline in an inhomogeneous atmosphere has its own Alfvén speed, the waves propagate at different speeds and soon become out of phase as you travel up through the atmosphere. Large spatial gradients build up until dissipation smoothes them out and extracts the energy. Thus, phase mixing naturally generates small scale motions and the fieldline will be heated only where the dissipation is important. However, once deposited, the heat will spread out along the fieldline by conduction.

Parker (1991) considers phase-mixing as a possible heating mechanism for coronal holes. However, he found that this mechanism could only provide heating at distances $\approx 10$ solar radii and thus this mechanism could only be used to accelerate the solar wind to high velocities.

\section{Other Wave-heating Methods}

An interesting idea that links both wave heating in the corona with the presence of many small reconnective sites in the coronal magnetic field (see Section 4.2.3) 


\begin{tabular}{|c|c|c|}
\hline & CDS & SUMER \\
\hline $\begin{array}{c}\text { Emission Line }(\AA) \\
\text { Spatial Resolution } \\
\text { (arc seconds) }\end{array}$ & $160-800$ & $500-1600$ \\
$\begin{array}{c}\text { Temporal Resolution } \\
\text { (seconds) }\end{array}$ & $1-10$ & $1-3$ \\
\hline
\end{tabular}

Table 4.2: Operating parameters for CDS and SUMER on SOHO

was explored by Lee and Roberts (1986). They investigated what happens when the local transverse oscillations produced by a passing Alfvén wave interacts with a tangential discontinuity in the Alfvén speed. They demonstrated that dissipation of the waves was possible when they propagated along a magnetic field containing a number of tangential discontinuities in the field direction (current sheets). Thus, the combination of the two processes leads to the possibility of a wave heating contribution even to the active corona.

\section{Observational Evidence of Coronal Wave Heating}

What evidence is there available of coronal Alfvén waves? It has been suggested that the dissipation of Alfvén waves could give a contribution to the heating mechanism of a coronal hole and large amplitude magnetic and velocity fluctuations have been identified in the solar wind (Hollweg, 1986 and references therein). This could simply be a remnant of a much larger coronal flux which may therefore be detectable.

Evidence for Alfvén wave heating can be found in the non-thermal broadening of transition region and coronal spectrum lines (for example, Hassler et al, 1990 and Narain and Ulmschneider, 1990 and references therein). The broadening is produced by a random velocity $\left(20-25 \mathrm{kms}^{-1}\right.$ in the quiet corona and $40-50 \mathrm{kms}^{-1}$ in active regions) which, for a reasonable value of the Alfvén speed, gives more than the required input energy flux.

The CDS and SUMER instruments aboard SOHO could give the most convincing evidence yet for Alfvén wave dissipation in the corona (see Ireland, 1995 for a short review on the capabilities of the SOHO Mission on observing wave heating). Table 4.2 shows the emission lines, spatial resolution and temporal resolution that the two instruments will be using. Possible tests for wave heating could be

1. The search for a hot sheath around a coronal loop produced by resonant absorption. Unfortunately, other mechanisms could also create this phenomena 
and so the test could be ambiguous.

2. The measurement of footpoint motions of the coronal field to see if the generation of Alfvén waves in the corona is even a possibility.

3. The emission line detection of a periodic oscillation in some physically measurable quantity.

4. The measurement of magnetic field fluctuations in a single coronal loop itself.

\subsubsection{MHD Turbulence}

Any driven MHD system can produce large gradients in the magnetic field and plasma itself. Turbulence then allows the energy to be transferred from large to small scales where dissipation can take effect and produce heating.

Plasma physicists have been considering the idea of MHD turbulence for some time. For example, Riyopoulos et al (1982) used a finite difference, resistive, MHD code to model both the decay to small scales (high wave number) and the growth to larger scales (low wave number), that is, an inverse cascade, of a "relaxing" turbulent ideal MHD system.

In the realm of Solar Physics, Van Ballegooijen (1986, 1987) showed that from an initially uniform magnetic field, a cascade of magnetic energy could occur in the corona as the result of a random motion imposed on the field lines by subphotospheric flows (see Section 4.2.3 for Parker's disagreement with this particular mechanism).

Heyvaerts and Priest (1992) considered turbulent heating of the corona by calculating the turbulent dissipation coefficients of a sheared coronal arcade under typical boundary stresses. They produced formulae for the heating rate and the turbulent velocity, the typical values calculated being comparable with observations.

This work was extended in a series of papers by Inverarity (Inverarity et al, 1995, Inverarity and Priest, 1995a, 1995b) to investigate turbulent coronal heating of sheared arcades, twisted flux tubes and the wave heating of coronal loops respectively.

To test the idea of MHD turbulent heating is very difficult mainly because the observational length scales are so small, possibly as low as $10 \mathrm{~m}$ (Van Ballegooijen, 1986; Inverarity, 1995, private communication). The Normal Incidence X-ray Telescope (NIXT) and Yohkoh observations (Goméz et al, 1993a, b; Martens and Goméz, 1992) have given indications of the existence of turbulent regimes in coronal active regions. Thus, it may be possible with $\mathrm{SOHO}$ to measure the temporal and spatial 
power spectra of line broadening within an isolated coronal loop but it should be noted that the turbulence produced by topological dissipation and by Alfven wave heating in the corona may produce a spectrum that is not very different from that of MHD turbulence.

\subsubsection{Microflares and Nanoflares}

In 1972, Parker demonstrated that if a fully three-dimensional coronal magnetic field exists, then, in ideal MHD, the static equilibrium must have a uniform twist along the field direction. However, in reality, such an equilibrium would not likely exist and tangential discontinuities in the field (current sheets) would form. Parker suggested that these were possible sites of Joule heating and reconnection.

Parker (1987b) continued this work by illustrating the spontaneous and unavoidable formation of tangential discontinuities in a force-free magnetic field whose footpoints are subjected to bounded, continuous displacement and shuffling, so that neighbouring flux tubes are wound and wrapped around each other in increasingly complicated patterns. Magnetic field reconnection occurs at the braiding boundaries creating heat and plasma flows (see Figure 4.1).

The hard X-ray balloon borne observations of Lin et al (1984) show intermittent spikes of duration $1-2$ seconds, the largest spike representing an energy release of $\sim 10^{27}$ ergs (termed a microflare as it is approximately $10^{6}$ times smaller than a typical large solar flare). An interesting aspect of the Lin et al data was that the microflares appeared to be composed of many thousands of smaller energy release events, right down to the detection limit of the observing instrument $\left(\sim 10^{24}\right.$ ergs per spike).

Parker (1988) used this as evidence to term the nanoflare; small, localised, impulsive bursts of energy corresponding to reconnection sites in the coronal magnetic field. Each microflare would then be made up of the superposition of many nanoflares. Parker also cited the work of Porter et al (1984) and Porter et al (1987) on the discovery of localised brightenings throughout the magnetic network of the corona and used them to estimate the characteristic nanoflare lifetime of $\sim 20$ seconds. Parker went as far as to say that the whole X-ray corona of the Sun ( and therefore of other similar solitary, late-type, main sequence stars) is created and sustained by these spontaneous and random tangential discontinuities in the surface magnetic field.

Parker (1993) extended his nanoflare hypothesis to include the creation of current sheets due to discontinuous fluid motions and found that this type of current sheet does not contribute significantly to the dissipation of free magnetic energy when a 


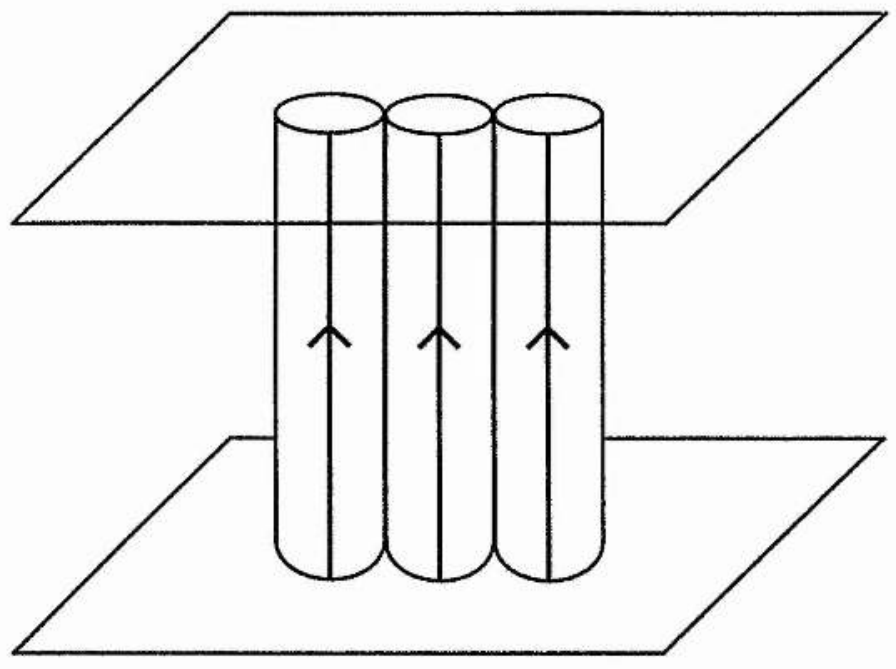

Initial Flux Tubes

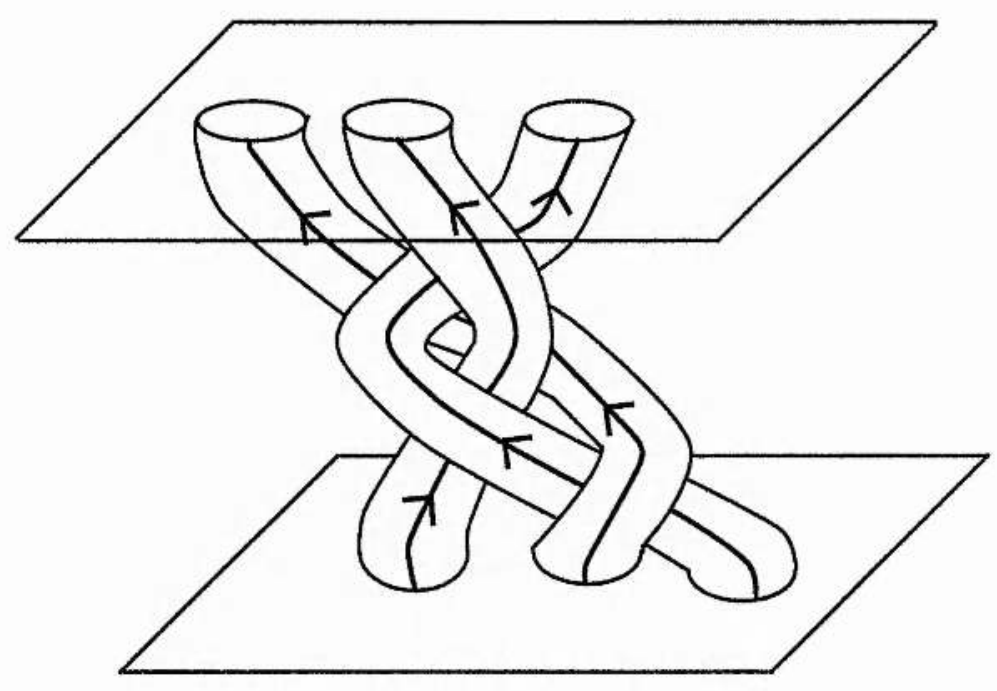

Braiding Effect

Figure 4.1: The braiding of magnetic flux tubes due to continuous footpoint displacement (Parker, 1987). 
small amount of resistivity is added to the system. He concluded that the topological dissipation must be the primary heating mechanism for the corona.

Parker's view of the inevitable formation of current sheets has been contested by Van Ballegooijen $(1986,1987)$ who instead suggested that the slow braiding of a magnetic field allows the development of steep gradients in an energy "cascade" process from large to small scales. He states that reconnection is not necessary and that the field can evolve through a series of equilibrium states. However, Van Ballegooijen's numerical simulation of this effective "shredding" of the coronal field into finer and finer elements was unable to reach a final equilibrium between the input at large scales and the dissipation at smaller scales. Also the energy output calculated from the cascade process was an order of magnitude less than the required energy input for an active region. Van Ballegooijen put this partly down to an underestimate in the random motions of the photospheric magnetic fields that he used.

Various authors have suggested that the controversy between the two methods is somewhat academic (Cargill 1994a; Zirker, 1993). Both mechanisms are indistinguishable from the viewpoint of the actual release of energy and the discussion has tended to simply concentrate on 2D magnetic fields. Priest and Forbes (1989) have shown that current sheets can form anywhere in a 3D field.

The idea of a "nanoflared corona" has received more attention over the last few years. Cargill (1993) considered a "point-model" of a loop to examine the implications on the corona of these small scale events. He devised a diagnostic called the filling factor (defined as the ratio of the coronal volume radiating $\mathrm{X}$-rays and EUV to the total volume considered) and concluded that it was slightly smaller than unity, that is, the corona can be assumed to be almost homogeneous.

Cargill (1994a, 1994b) extended the above idea to investigate an analytical model of a large scale coronal structure comprising of many hundreds of small elemental flux tubes. The magnetic field was assumed to be so strong that each elemental loop acted as an isolated atmosphere and the loop cooling was modelled by two specific stages; (1) immediately after impulsive heating, the loop cooled solely by conduction and then at some critical temperature; (2) it cooled solely by radiation. Cargill's results showed that for a variety of loop lengths in a given area, the temperature and density distributions calculated were around commonly accepted values for an active region corona. However, he was unable to reproduce the increase in the emission measure with decreasing temperature below $10^{5} \mathrm{~K}$.

Hudson (1991) showed that if the nanoflare coronal heating theory is correct, then nanoflares cannot have the same power-law distribution function against energy as 
other larger solar flares. Extrapolating the observed rate of flares down to nanoflare energies, Hudson found that they could not fulfill the energy losses of the corona. If nanoflares are responsible for coronal heating then they must satisfy a different distribution function and may, in that case, operate under different physics than other flares.

Kopp and Poletto (1993) developed a simple semi-analytical model to describe the temporal evolution of a nanoflared plasma. They argued that since existing instrumentation is unable to resolve the spatial dependence of nanoflares within the structure of individual loops, spatially averaged loop properties are sufficient rather than using complicated and cumbersome global numerical codes. They investigated very short coronal loops $(2-5 \mathrm{Mm})$, arguing that individual nanoflares would only be detectable in loops of these lengths.

Kopp and Poletto agree with Hudson (1991) that nanoflares must obey a distinct power law in order to balance the coronal energy losses. Setting the power index at 4 , they estimated the nanoflare generation rate at $4.1 \times 10^{5} \mathrm{~s}^{-1}$ for the entire corona and demonstrated that a hot plasma $\left(\sim 10^{6} \mathrm{~K}\right)$ could be created from an originally cool, evacuated loop that undergoes repetitive nanoflaring.

Perhaps the closest work to this thesis has been carried out by Peres et al (1993) who presented results on hydrodynamic simulations of coronal loops maintained close to stationary conditions by a sequence of microflares. Using the PalermoHarvard Hydrodynamic model for coronal plasma confined in loops (see Peres et al, 1982), they simulated microflares as small heating episodes which account for all the heating present.

To model the release of microflare energy, they investigated a periodic sequence of identical and short pulses as well as a sequence of random pulses of variable intensity and duration. The intensity of each pulse was based on a "load-and-release" mechanism. In the random case, the time sequence of events and their duration was random, the total energy delivered at each pulse equalling that necessary in the analogous stationary loop case; that is, the energy released over the time between the end of the previous pulse and the end of the on-going one.

Their results showed that a loop could be maintained at coronal values provided that the characteristic heating repetition time is of the order or less than the characteristic cooling time, $\tau_{\text {cool }}$, which they define as

$$
\tau_{\text {cool }}=120 L_{9} T_{7}^{-0.5}
$$

where $L_{9}$ is the loop semi-length in units of $10^{9} \mathrm{~cm}$ and $T_{7}$ is the loop apex temperature in units of $10^{7} \mathrm{~K}$. They also suggest that a diagnostic for variable heating may be possible. For example, although variations in line intensity measurements may 
be difficult to detect, ratios between lines for steady heating would be very different from those expected with variable heating.

Vlahos et al (1995) introduced something called the "statistical flare". They assume that the coronal active region is very inhomogeneous with a large number of magnetic loops interacting with each other and randomly forming discontinuities in many independent spatial points. The system is driven by turbulent motions in the convection zone and photosphere. When a single discontinuity (reconnective event) occurs, it triggers off other events forming an avalanche effect. A flare is then defined as a swarm of these events happening almost simultaneously in a localised region. This scenario is different from Parker (1988) who suggested that flares (microflares) were made up from the superposition of microflares (nanoflares). Valhos et als results agree with Hudson (1991) - a power-law index of $\sim 3.5$ was calculated for the low energy, nanoflare region while a lower index of $\sim 1.8$ was found for the higher energy region, the latter value agreeing with current observations.

\section{Observations of Microflares and Nanoflares}

Following on from Lin et al (1984), there has been a recent "explosion" in the search for observational evidence of small scale energy release events in the corona. Schmeider et al (1994) conclude from observations that the simplest microflare may be composed of a large number of smaller events and that it is likely that the events are triggered successively.

By overlapping vector magnetograms from the Marshall Space Flight Center (MSFC) Solar Vector Magnetographs with the same observations of bright coronal X-ray features from the Soft X-ray Telescope (SXT) on Yohkoh, Moore et al (1994) found enhanced coronal heating at sites of strong magnetic shear, the heating itself continually fluctuating in the manner of an irregular sequence of microflares. They concluded that magnetic shear helps the heating process but does not provide all the heat energy alone.

A lot of work on what has been termed "active region transient brightenings" has been carried out by Toshifumi Shimizu (Shimizu et al, 1992; Shimizu, 1995). Again using SXT observations, Shimizu discovered many frequent flare-like brightenings, mostly in the form of single and multiple loops (for multiple loop brightenings see Section 4.2 .4 on coronal loop interactions), the loop heating occurring at either the footpoints or at the loop intersection. Table 4.3 lists the physical parameter value ranges of a typical transient brightening as found by Shimizu.

Shimizu calculates a power-law distribution that appears to extend from the solar flare range down to an energy of $10^{27}$ ergs. As in Hudson (1991), he states that if 


\begin{tabular}{|c|c|}
\hline Physical Parameter & Observed Values \\
\hline Temperature & $4-8 \times 10^{6} \mathrm{~K}$ \\
Volume Emission Measure & $10^{50.5}-10^{53.5} \mathrm{~m}^{-3}$ \\
Electron Density & $2-20 \times 10^{15} \mathrm{~m}^{-3}$ \\
Gas Pressure & $0.5-2$ Pascals \\
Loop Length & $0.5-4.0 \mathrm{Mm}$ \\
Loop Width & $0.2-0.7 \mathrm{Mm}$ \\
Duration & $2-7$ minutes \\
Total Energy Loss & $10^{18}-10^{22}$ Joules \\
\hline
\end{tabular}

Table 4.3: Range of observed physical parameter values for a typical transient brightening (Shimizu et al, 1992; Shimizi, 1995).

this power law continues down to lower energies, transient brightenings would not be able to provide enough energy to balance coronal losses. However, the author proposes that it is still possible that these energy release events may still make a significant contribution to coronal heating as

1. an accurate frequency distribution below $10^{27}$ ergs has not yet been obtained;

2. UV microflares have been observed more frequently than hard X-ray microflares (Porter et al, 1995). Thus the transient brightenings observed by SXT may only be part of a much wider range of energy events;

3. other energetic terms (for example, plasma flows) may make additional contributions to the heating - they were not considered in the Shimizu et al study.

So far individual nanoflares ( $\sim 10^{24} \mathrm{ergs}$ ) have yet to be observed directly. This could mean that either the temporal variations of the loop properties as a result of a nanoflare energy event are observationally insignificant or that the variations occur on a scale that is beyond the scope of current instrumentation. For example, it is not possible as yet to resolve a $2 \mathrm{Mm}$ coronal loop (as considered in Kopp and Poletto, 1993). The pixel size on SXT is $3 " \times 3$ " and if we assume that the imaged area is completely filled with flux tubes of length $4 \mathrm{Mm}$ and diameter $0.4 \mathrm{Mm}$, then approximately six of the flux tubes will fill an image pixel. It would be simplistic to say that individual nanoflares in individual loops could be detected as it is more than likely that a continuous flickering on and off of the entire region will take place as a large number of events would be occuring in order to keep the plasma from cooling down. 
The small dissipation scales in the corona make it very difficult to see the actual energy release events happening. Thus, it is up to the theorists to see whether a unique coronal heating signature can be calculated (such as the filling factors of Cargill, 1993). Searching for nanoflares was the subject of a paper by Zirker and Cleveland (1994). They suggested that nanoflares may be too weak to detect the impulsive energy release and may occur too frequently to be adequately resolved. However, nanoflares do produce impulsive flows in coronal loops which, if detected, would give strong evidence for the nanoflare hypothesis. Believing that individual loops will eventually be resolved and that NIXT, with its arc-second resolution will be improved in the near future, Zirker and Cleveland conclude that if a $10^{24} \mathrm{erg}$ energy burst exists, then NIXT could detect the transverse motion of resulting hot plasma shocks in loops above the solar disk.

Other possible ways of detecting microflares and nanoflares could include the following (Hudson, 1991, 1994):

- They may be observed individually as samples, sticking out like "sore thumbs" in the time series.

- They may appear only statistically as a general property of the time series. Analysis of many events simultaneously will decrease the high frequency fluctuations due to counting statistics and may reveal the presence of small bursts of energy, provided that they have the same statistical properties of other flares.

- They may be detected as the result of correlation with other indicators at different wavelengths. However, a large increase in instrument sensitivity at all wavelengths would be required (Zirker, 1993).

The extension of the idea of a nanoflared corona to stellar coronae has been considered theoretically (Parker, 1993) and observationally (Butler et al, 1986) on $\mathrm{dMe}$ and possibly all late-type stars and although Yohkoh observations have shown the way forward, the Solar community can only look forward to instruments on $\mathrm{SOHO}$ to maybe finally resolve the nanoflare problem.

\subsubsection{Coronal Loop Interactions}

In active regions, the concentration of individual magnetic flux bundles inevitably leads to the situation where a portion of one particular loop comes into contact with an adjacent loop to the extent that they interact and leave an observable brightness at their interaction site. Coronal loops can be twisted due to photospheric motions (Parker, 1988) leading to the generation of longitudinal currents along the loops. 
These currents in inclined and colliding loops can lead to partial reconnection and the release of energy at the interaction site. This process has been termed a coronal loop interaction (CLI), clear examples of which have been observed by Yohkoh (Shimizu et al, 1992).

Airapetian and Smartt $(1994,1995)$ have investigated the theoretical considerations of CLI's and found that they appear to represent a different type of impulsive, energy release event in the corona. They differ from flares in that

1. Transient brightenings due to CLI's are the result of the encounter of two or more loops compared with flares where the final energy release is due to the gradual accumulation of stored energy.

2. The typical heights ( $\sim 40 \mathrm{Mm})$ at which CLIs occur appear to be much higher than characteristic flare heights in the corona.

3. The typical energy release due to a CLI is $1-5 \times 10^{26} \mathrm{ergs}$, the size of a small microflare.

4. CLIs show highly compressed and hot $\left(5-6 \times 10^{6} \mathrm{~K}\right)$ enhancements radiating soft $\mathrm{X}$-rays while the SXR emission due to a solar flare appears in the low corona with a typical temperature of $\sim 10^{7} \mathrm{~K}$ with little or no plasma compression occurring.

From the Yohkoh data, Airapetian and Smartt calculate a repetition time for CLI events at $\sim 200$ s. Optical observations give a characteristic cooling time of the plasma at an interaction site as $\sim 480-600$ s, thus suggesting that CLIs could be a possible source of significant coronal heating. They calculate the frequency of CLIs in an active region as

$$
\tau_{\mathrm{cli}} \approx 12 \gamma^{2} \text { events per minute }
$$

where $\gamma$ is the fraction of the total flux that emerges from the photosphere into the corona. Thus, their coronal energy model and the number of events occurring, will vary with the solar cycle - an important aspect when considering coronal heating.

Airapetian and Smartt conclude that CLIs could heat the corona in the form of discrete energy releases on the scale of microflares. The magnetic energy released would heat the plasma, create flows and accelerate energetic particles. Magnetoacoustic waves could also carry energy away from the interaction site (Porter et al, 1994a). 


\subsubsection{Why Time-Dependent Heating?}

In this chapter a time-dependent heating term is investigated because none of the proposed heating mechanisms can provide a constant supply of thermal energy to the corona.

The energy source for the process of resonant absorption is a wave propagating in from the photospheric boundary. This wave is generated by random convective motions and its frequency will therefore continually vary. A given fieldline will change from being resonant to non-resonant, the fieldline only being heated when resonance occurs. Thus the heat source will vary in time.

Phase-mixing and MHD Turbulence are created due to the oscillation of the coronal loop photospheric footpoints and the varying Alfven speed in the corona. There is no reason why these footpoints should continue to be moved at the same frequency; it is a random process. As the frequency varies, so will the position and the amount of heat deposited. Thus a time-dependent heating supply must be modelled.

Heating by nanoflares and coronal loop interactions appear to be the most obvious time-dependent thermal energy sources. Heating will only occur while there is still field left to reconnect. Once reconnection has finished, there will be no more heating until another current sheet forms. Thus, the release of impulsive, random, discrete energy bursts into the corona can only be realistically modelled by using a time-dependent heating term.

A heating term that varies in time has been considered by other authors. Mariska et al (1982) introduced a numerical model to investigate the transition region response to variations in the energy input. Using a vertical flux tube model with constant gravity, they found that when the volumetric heating was switched on and off, the thin laminar transition region structure did not appear to change. No thickening of the region occurred; it simply slides up and down on top of the cooler chromosphere. It should be noted that Mariska et al argue against simply treating the chromosphere as a mass source or sink. They describe the chromospheric region as behaving like a loaded spring with the overlying atmosphere acting as the load. Thus changes in pressure will cause the chromsphere to rise and fall accordingly, this part of the Sun then being defined by a particular temperature $\left(\sim 10^{4} \mathrm{~K}\right)$ rather than at a specific height.

The thermal stability of coronal loops was examined by Craig et al (1982) by allowing a loop to be heated by a low amplitude, additional energy input and its subsequent dynamic relaxation followed. They found that the loop structure critically depended on the temperature profile at the base of the transition region which 
therefore required very high spatial resolution in that part of the loop. They also argued that the loop structure was unique in that after the energy input, the loop relaxed to a state that was physically indistinguishable from its initial configuration.

Peres et al (1982) investigated the stability of "magnetically confined atmospheres". They examined mild perturbations in the temperature and density of an initially relaxed atmosphere to see if these variations could destroy the initial static state as well as test the stability of the numerical code; the loop was found to be stable to finite amplitude fluctuations. The authors then examined three cases of atmospheric response to weak changes in the loop heating rate where the heating was (i) in the corona alone; (ii) in the upper chromosphere alone and (iii) along the entire length of the loop. They found that increases in the coronal density and temperature that agreed with observations could only be achieved by preferentially dumping the heat in the chromosphere rather than the corona.

A flare-loop model suggested by MacNeice (1986) described the response of a fully ionised plasma in a coronal loop subject to a transient heating pulse superimposed on a constant background heating and centred about the loop apex. The author used an adaptive grid approach in the numerical scheme to simulate the impact of a downward propagating conduction front from the corona onto the transition region due to the pulsed heating term.

Mariska (1987), extending the work of Mariska et al (1982), considered the response of an initially static coronal loop structure to large decreases and increases in the heating rate. His aim was to try and reproduce the observations of Doppler shifts in UV emission lines formed in the transition region which show continual plasma downflows and impulsive plasma upflows. Mariska decreased the value of the heating term to $10 \%$ and $1 \%$ of its initial value and then, once a new equilibrium had been reached, increased the heating again to its original value. He concluded that a loop that is cycling between cooling and heating had difficulties in producing the velocity mass motions of the order observed in the solar transition region, as well as the emission curve below $10^{5} \mathrm{~K}$.

Loop heating rate variations with respect to time have been investigated by Brown (1995) in order to explain the existence of arcs of plasma in active regions with temperatures in the range of $10^{5}-10^{6} \mathrm{~K}$. The intermediate temperature loops have much higher densities than those predicted by static loop models and the short lifetimes and the large velocities observed in such loops point towards the need for a dynamic heating model. Previous calculations placed a mass source at the loop apex to produce loops with increased density profiles over their static counterparts but it is difficult to see how this mass could be added to the loop apex in the first place. 
Instead, Brown numerically simulates the response of a loop to a reduction in the heating rate to $10 \%$ of its original value and demonstrates that the loop temperature and density values pass through typically observed intermediate temperature loop values.

Sturrock et al (1990) examined a spicule-type model of the corona, where the solar atmosphere is heated episodically by short bursts of energy followed by long periods of radiative cooling. They calculated the differential emission measure (DEM) curve created by such a scenario and were able to model the negative (positive) gradient part of the curve below (above) $10^{5} \mathrm{~K}$. The necessary injection rate of energy was similar to that observed for spicules though it must be noted that their model ignored the role of thermal conduction in the plasma. We will return to the effect of time-dependent heating upon DEM curves later on in this chapter.

Various authors have considered time-dependent heating processes in other stars. Mullan and Cheng (1994) used a time-dependent hydrodynamic code to follow the propagation of acoustic waves into the corona of an $\mathrm{M}$ dwarf star. An important difference between this type of star and the Sun is that the acoustic spectrum in $M$ dwarfs is expected to peak close to the acoustic cut-off and thus allow for a much more effective transmission of acoustic power into the stellar atmosphere.

Mullan and Cheng posed the question: Can acoustic wave heating maintain an initial atmosphere which contains a hot gas? Beginning with an initially steady state $\mathrm{M}$ dwarf atmosphere, the authors time-dependent approach followed the injection of acoustic waves at certain periods at the lower boundary, their propagation upward through the atmosphere and their deposition of heat as the waves non-linearly evolved. They found that acoustic heating of coronae could be at work amongst the most inactive of $\mathrm{M}$ dwarf stars. Acoustic heating could not create a corona but simply maintain it. Some other non-acoustic mechanisms must bring about the initial temperature rise.

Sion (1995) was the first to consider the evolutionary thermal response of a white dwarf star to the periodic deposition of mass by a dwarf nova accretion event. The white dwarf responds to the compression produced by the weight of the added material by heating up and then cooling down very rapidly. He numerically simulated this process by switching on spherical accretion for the duration of a dwarf nova outburst interval and then by switching the accretion off and following the cooling of the star until the onset of the next outburst. For simplicity it was assumed that the accreting material "landed softly" onto the stellar surface with zero velocity and with the same temperature and density as the matter already present at the surface.

His results showed that if the thermal timescale (the time it takes for a cer- 
tain amount of heat deposited in the star to "spread out") of the heated region is longer than the interval between accreting outbursts and if the ratio of the outburst timescale to the quiescence timescale (between outbursts) is in the range $0.2-0.5$, then compressional heating can be a considerable source of heat energy in a white dwarf.

In this chapter, we will use the coronal loop model defined in Chapter 3 but vary the value of the heating term in time to see if we can maintain the coronal plasma at typical coronal temperatures. Thus, an isobaric assumption to the coronal plasma is considered; the reduced set of isobaric equations (2.38)-(2.40), derived in Chapter 2 , allow one to follow the thermal evolution without the restriction of tracking the propagation of the sound waves. This has a great computational advantage compared with the numerical codes mentioned above (for example, Mariska et al, 1982). The slower thermal variations can be followed with timesteps only restricted by the physical processes that we wish to investigate. Thus, with the minimum amount of computing time and power, it is possible to follow the long time behaviour of the response of the plasma to variations in the heating term.

\subsection{The Isobaric Equations and the Time-Dependent Heat- ing Term}

The reduced, dimensionless isobaric equations to be investigated are

$$
\begin{gathered}
\frac{\partial \rho}{\partial t}+\frac{\partial(\rho v)}{\partial x}=0 \\
\frac{\partial v}{\partial x}=\frac{\partial}{\partial x}\left(T^{5 / 2} \frac{\partial T}{\partial x}\right)-b\left[\chi T^{\alpha-2}-H\right] \\
\rho=\frac{1}{T}
\end{gathered}
$$

as derived in Chapter 2 with boundary conditions (2.41), (2.42) and initial conditions (2.43), (2.44) and with the values for $\chi$ and $\alpha$ obtained from the Two Range Radiative Loss Function (3.3).

Wright and Rickard (1995) found that for a one-dimensional MHD cavity driven by a prescribed random boundary motion, the ohmic heating followed a timedependent pattern similar to a sine wave. Thus, in the first instance, the effects of a sinusoidal heating function

$$
H(t)=H_{0}(1+\sin \omega t)
$$


will be investigated, where $H_{0}$ is the average amount of heat deposited in the corona and $\omega$ is a frequency which will be varied. Note that there is no spatial dependence so that every point on the field line receives the same amount of heat. This is reasonable since for high temperatures the conductive timescale can be quite short. The numerical algorithm developed for these equations can be found in Appendix F.

\subsection{Analytical Test of Numerical Code for Time-Dependent Heating of an Initially Uniform Atmosphere}

One possible test of the numerical code is to consider the effect of the heating term (4.6) on an initially isothermal atmosphere. If we have $H_{0}=1$, then $T(x)=1$, with $T_{e}=1$, is a solution to the equilibrium equation discussed in Section 3.2. A large $\omega \gg 1$ analysis can be carried out on equations (4.3)-(4.5), assuming that $T>T_{r}$ at every stage of the evolution. Thus, equation (4.4) reads as

$$
\frac{\partial v}{\partial x}=\frac{\partial}{\partial x}\left(T^{5 / 2} \frac{\partial T}{\partial x}\right)-T^{-7 / 4}+1+\sin \omega t,
$$

where $b=1$ for simplicity. The equations can be linearised about $T=1$ and an analytical solution can be derived (see Appendix C);

$$
\begin{aligned}
T(x, t)=1 & +\frac{1}{\omega}\left[-\cos \omega t+\mathrm{e}^{X_{1}} \cos \left(X_{1}+\omega t\right)+\mathrm{e}^{-X_{2}} \cos \left(X_{2}-\omega t\right)\right] \\
& +\frac{1}{\omega} \sum_{n=0}^{\infty} \frac{(-1)^{n}}{(2 n+1) \pi} \cos [(2 n+1) \pi x] \mathrm{e}^{-\left[(2 n+1)^{2} \pi^{2}-7 / 4\right] t}
\end{aligned}
$$

where

$$
X_{1}=\left(\frac{\omega}{2}\right)^{1 / 2}(x-1 / 2)
$$

and

$$
X_{2}=\left(\frac{\omega}{2}\right)^{1 / 2}(x+1 / 2) \text {. }
$$

The analytical results can be compared with the numerical calculations for the summit temperature $(T(0))$. In this case, (4.8) reduces to

$$
\begin{aligned}
T(0, t)=1 & +\frac{1}{\omega}\left[-\cos \omega t+2 \mathrm{e}^{-\frac{1}{2}(\omega / 2)^{1 / 2}} \cos \left(-\frac{1}{2}\left(\frac{\omega}{2}\right)^{1 / 2}+\omega t\right)\right] \\
& +\frac{1}{\omega} \sum_{n=0}^{\infty} \frac{(-1)^{n}}{(2 n+1) \pi} \mathrm{e}^{-\left[(2 n+1)^{2} \pi^{2}-7 / 4\right] t} .
\end{aligned}
$$




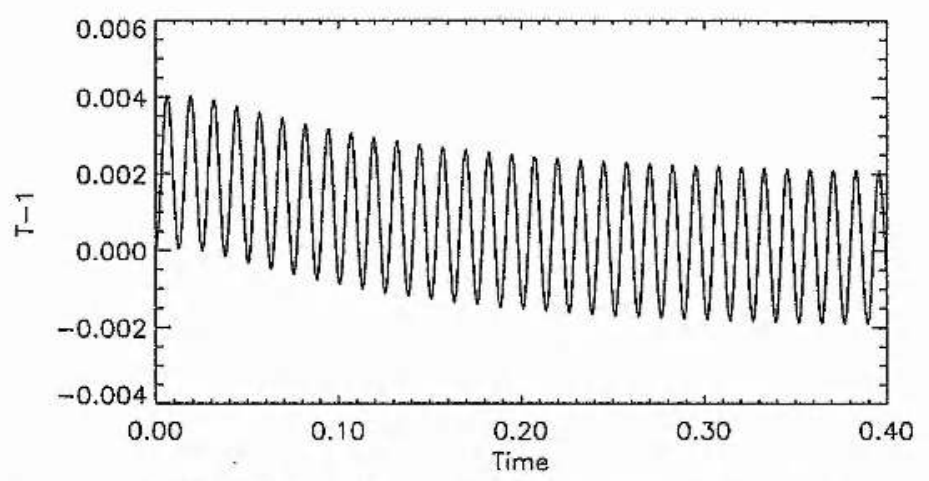

Figure 4.2: Evolution of summit temperature of the loop for $\omega=500$.

Figure 4.2 shows the variation in $T(0)$ for $\omega=500$ produced by the numerical code and Figure 4.3 shows the difference between the analytical $\left(T_{0}\right)$ and the numerical $\left(T_{n u m}\right)$ results for two different sizes of grid (grid-spacing $\delta x=0.05$ (black line) and $\delta x=0.025$ (green line)) - the summation in (4.11) has only been calculated to the fourth term. The error between the results decreases with decreased gridspacing. There is a slightly bigger difference between the results at the beginning of the run. This is simply due to the fact that the analytical calculation has only been taken to the fourth term in the series. Including more terms decreases this error. It should also be noted that the long time evolution of the summit temperature, that is, $(1-\cos (\omega t) / \omega)$, is out of phase with the heating term, $(1+\sin \omega t)$.

Thus the numerical code appears to reproduce the correct evolutionary behaviour of the model equations for this simplified system.

\subsection{Time-Dependent Heating of an Initially Static Loop}

Consider $b=0.5$ (corresponding to a loop length of $\approx 60 \mathrm{Mm}$ ) and $H_{0}=50$, an average heating value which produces a steady state summit temperature at coronal values for this particular value of $b$ and corresponds to an energy release of $\approx 2 \times 10^{25}$ ergs $^{-1}$ (this compares very well with the estimates given by Kopp and Poletto (1993) for the energy deposited in this length of loop by successive nanoflaring to maintain coronal temperatures) with $T_{e}=0.01$ and $T_{r}=0.011$ (both in units of $\left.10^{6} \mathrm{~K}\right)$.

Figure 4.4 shows the evolution of the temperature along half the loop for $\omega=7.5$; 


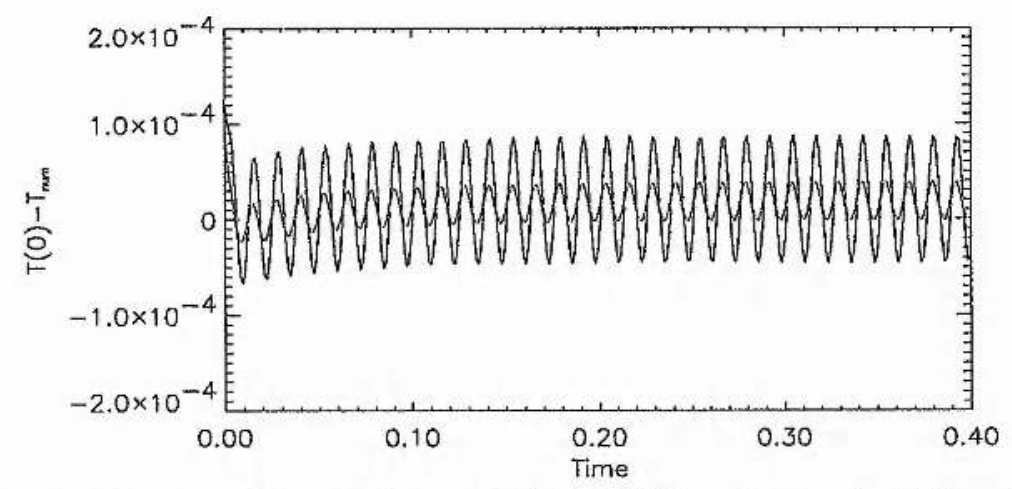

Figure 4.3: Error between the analytical $(T(0))$ and numerical $\left(T_{n u m}\right)$ for $\omega=500$.

note that a hot corona is maintained but there is a substantial variation in the temperature. Figure 4.5 shows the evolution of the heating function for the same timescale. The loop temperature initially rises (as the heating function increases away from its steady state value) causing plasma to flow out of the loop. As the heating decreases below $H=H_{0}$, the temperature along the loop falls (the summit temperature cools to half its original value) and, as time progresses, a "cool" region forms, extending from the footpoint towards the summit of the loop as cool, dense plasma rushes up the loop legs. As $H(t)$ increases from its minimum value of zero, this cool region is reduced and the cool plasma drains back onto the chromosphere a typical coronal loop temperature structure, similar to the static state, is recovered. This cycle is repeated the velocity of the cool material ranging from $10-100 \mathrm{kms}^{-1}$. Heinzel (1994) reported on observations of what he termed " $\mathrm{H} \alpha$ " loops which were basically hot but have cooler material $\left(\sim 2 \times 10^{4} \mathrm{~K}\right.$ and therefore visible in the $\mathrm{H} \alpha$ line) in one or both legs that is observed moving up and down (Figure 4.6). The scenario described above could explain these observations.

Also, this variation of temperature along the loop length would show up as periodic changes in the intensity of X-ray observations of the corona. The size of the temperature variation is dependent on the frequency of the heating - the higher the frequency, the smaller the variation. A slight change in the X-ray intensity would be consistent with a large frequency oscillation in $H(t)$, whereas the variations in impulsive brightenings of between $20 \%$ - 100\% observed by Porter et al, (1984) would suggest a smaller heating frequency.

Since the idea is to maintain a hot corona, it is only necessary to concentrate 

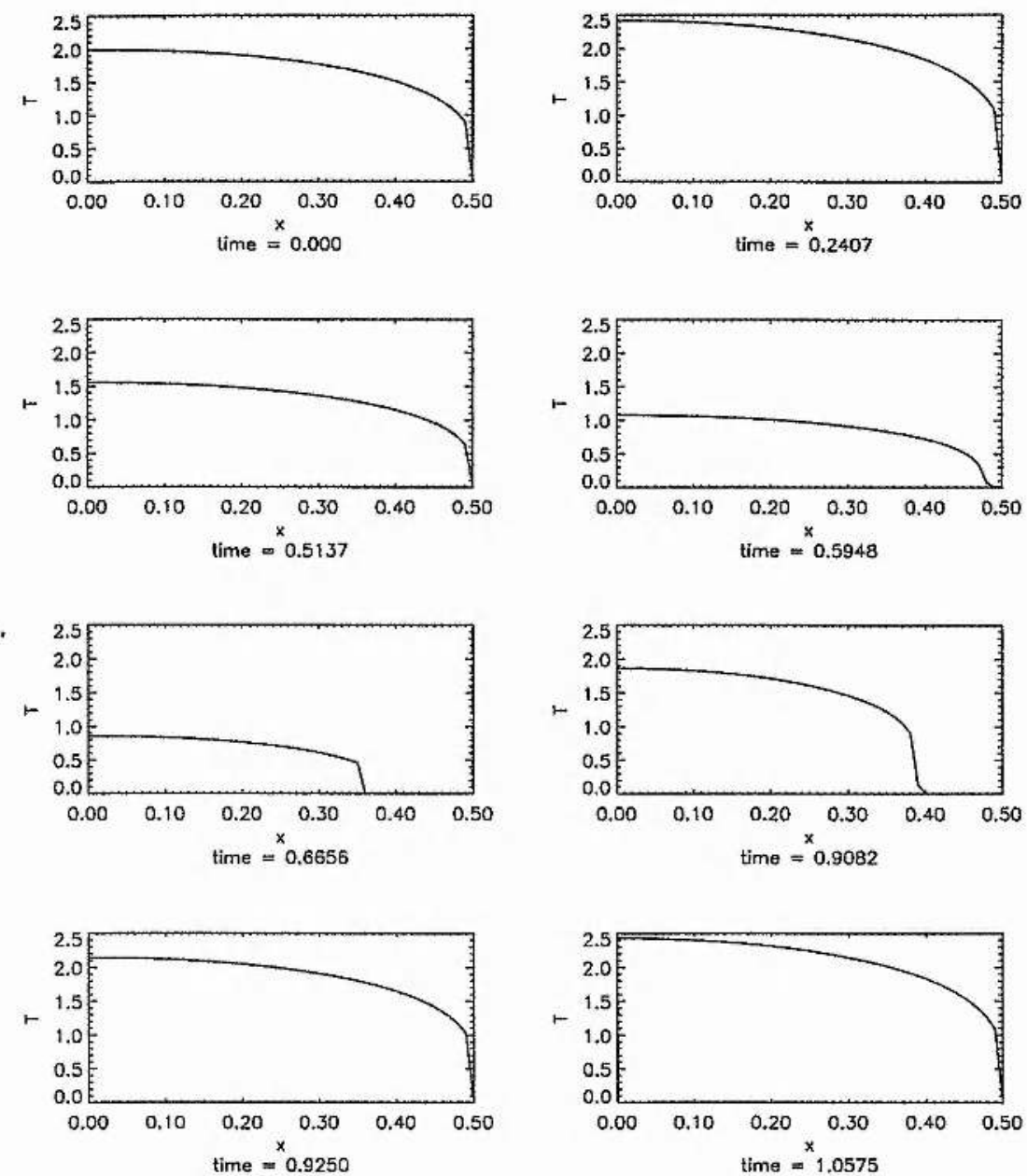

Figure 4.4: Evolution (in non-dimensionalised time units) of temperature along the half-loop length for $b=0.5$ and $\omega=7.5$.

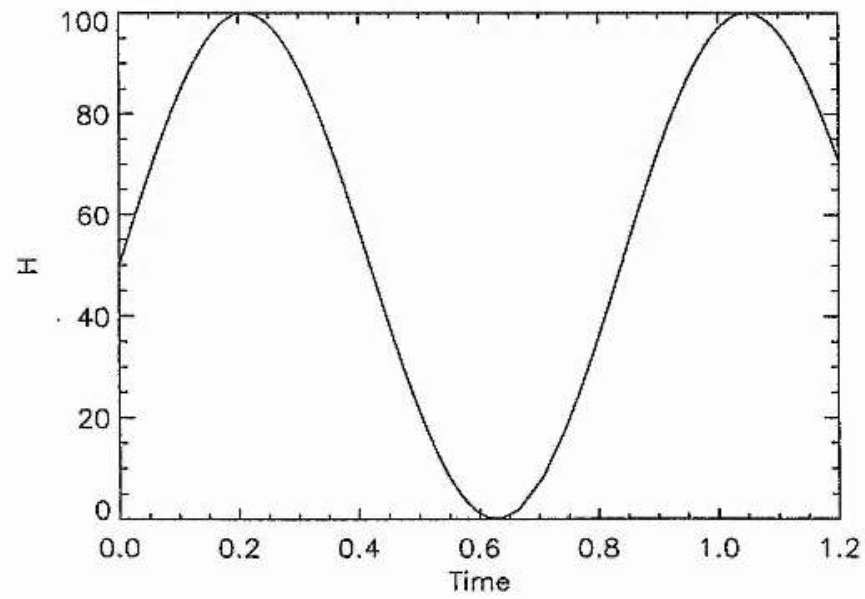

Figure 4.5: Evolution of heat deposited in the loop for $\omega=7.5$. 


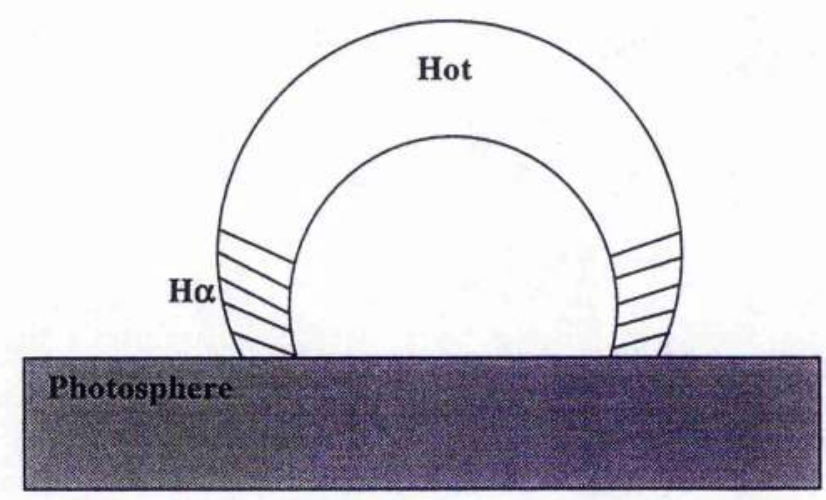

Figure 4.6: $\mathrm{H} \alpha$ Loop as shown in Heinzel (1994).

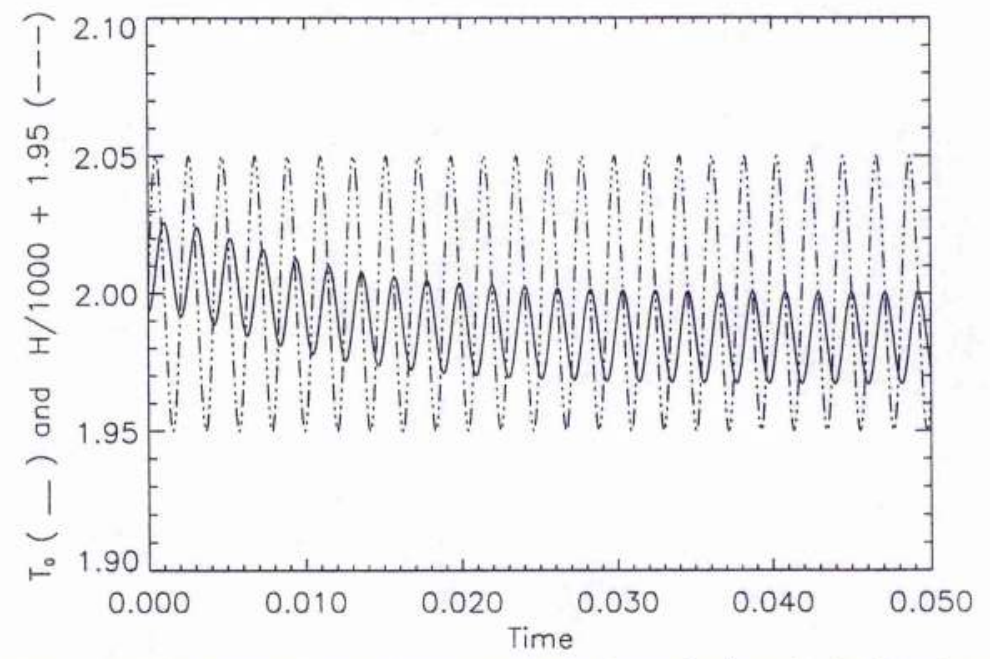

Figure 4.7: Evolution of summit temperature of the loop (-) and the heating function (with an appropriate scaling) (- - ) for $\omega=3000$. 


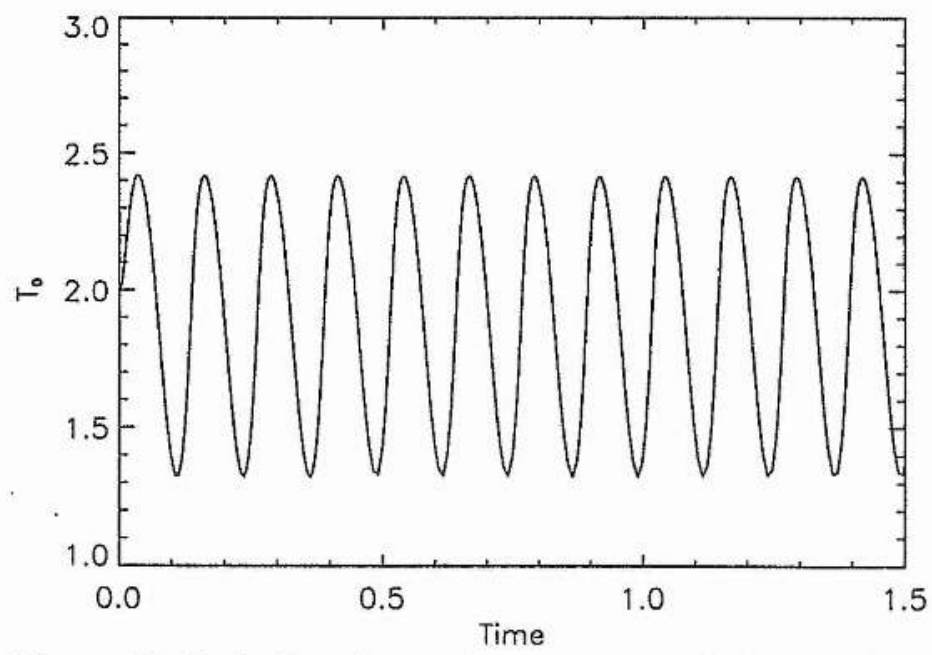

Figure 4.8: Evolution of summit temperature of the loop for $\omega=50$.

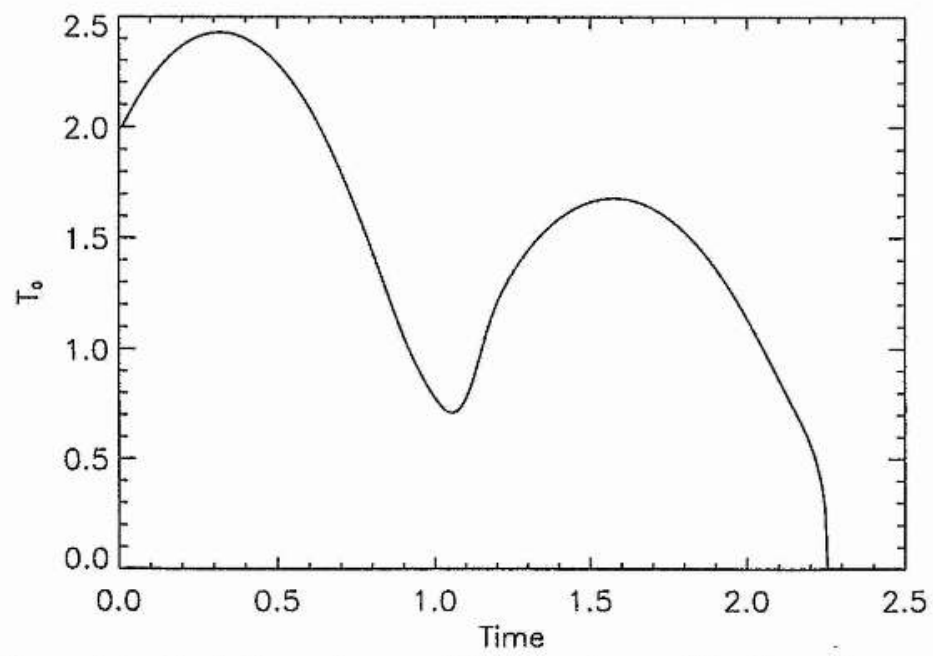

Figure 4.9: Evolution of summit temperature of the loop for $\omega=5$. 


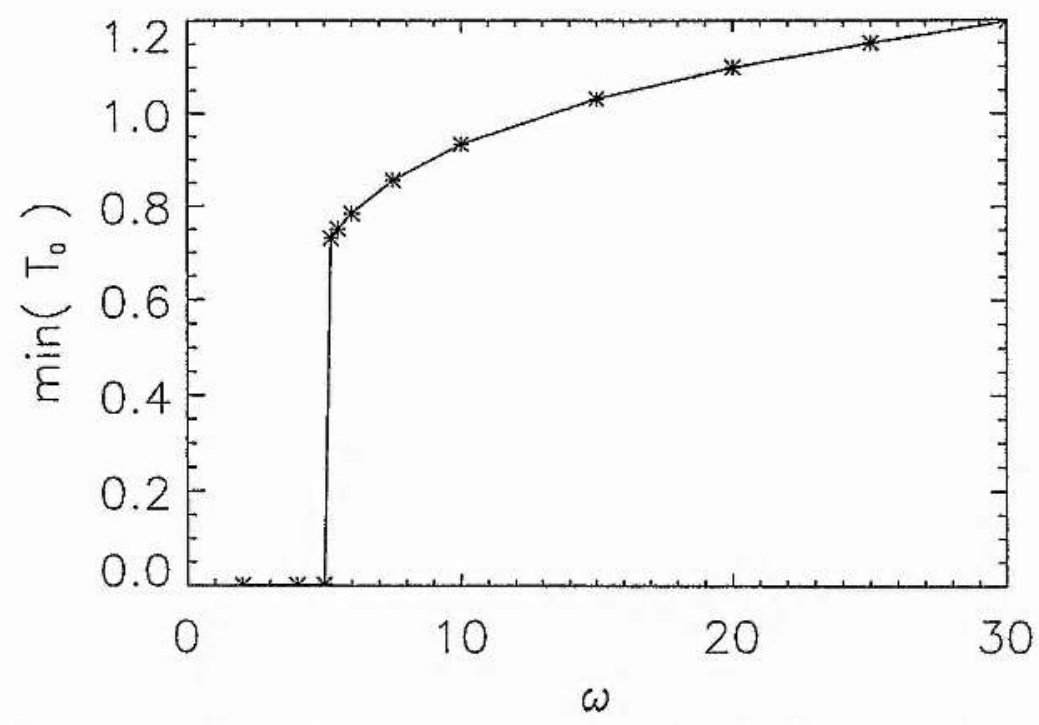

Figure 4.10: The minimum summit temperature against the frequency of heating function.

upon the evolution of the summit temperature to see if it can be kept at coronal values. Thus, Figures 4.7 to 4.9 shows the evolution of $T_{0}$ with respect to time for $\omega=3000,50$ and 5 respectively.

When $\omega=3000$ (Figure 4.7), there is only a small variation in the temperature from its steady state value. After a short time, the temperature settles down to a steady oscillation and there is a phase difference of $\pi / 2$ between the heating and the plasma temperature response which is consistent with the large $\omega$ calculations in Section 4.4 where the heating term followed a sine function whereas the long time temperature variation was predominantly cosine.

For $\omega=50$ (Figure 4.8), the amplitude of the oscillation is larger than for $\omega=$ 3000 and should now be observable while for $\omega=5$ (Figure 4.9), the temperature initially rises, then falls as the heating decreases to zero; it starts to recover to coronal temperature values as the heating increases again but in the next cycle the temperature collapses to chromospheric values.

Thus, there is a frequency above which coronal temperatures can be sustained and below which a cold plasma forms. The existence of such a critical frequency $\left(\omega_{\text {crit }}\right)$ is shown Figure 4.10. If the frequency dropped and the loop cools along its length, increasing the frequency again would not return the plasma temperatures to coronal values; the temperature along the loop would simply oscillate about the lower isothermal states. For the particular parameter values used, $H_{0}$ would have to exceed $\sim 10^{3.43}$ to recover a hot corona. This value corresponds to only a hot solution existing in the steady state phase plane (see Section 3.3). Such a situa- 


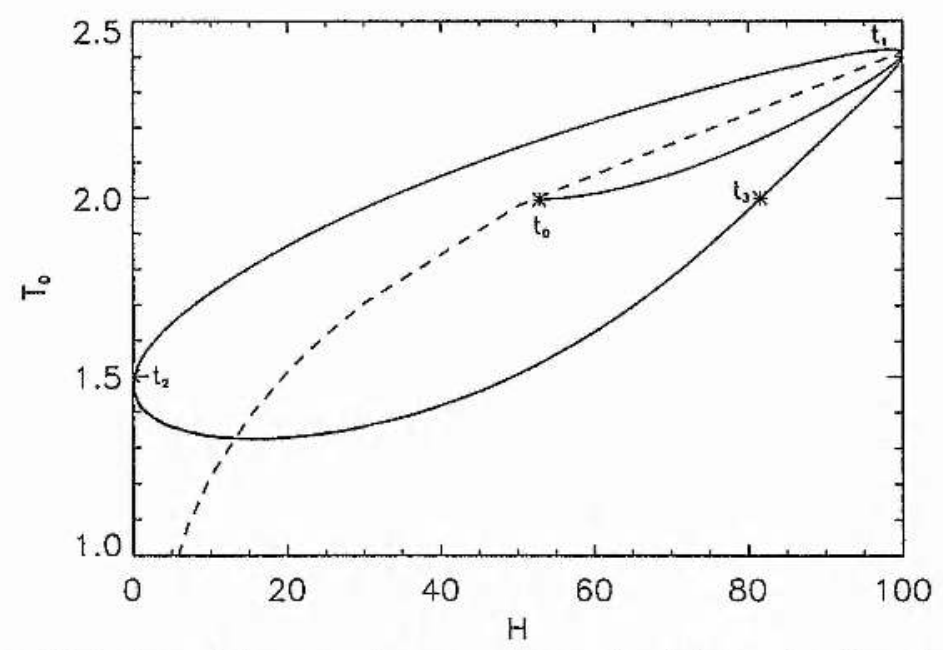

Figure 4.11: Variation of the summit temperature $T_{0}$ with heating function $H$ for $\omega=50$.

tion might arise as a result of the process described by Glasgaard and Nordlund (1995) who investigated the response of an initially homogeneous magnetic field to random shear motions on two boundaries. They found that as the field lines began wrapping themselves around each other into increasingly complex patterns, the joule dissipation initially showed a rapid rise and then fluctuated about a steady value.

If $\omega<\omega_{\text {crit }}$, then after a time, cool, dense plasma would form in the hot, rarefied corona. Hence a possible mechanism for the initial formation of a prominence is that the frequency of heating, and not the average amount of heat deposited, is reduced below the critical value. Fiedler and Hood, 1993, discuss a similar problem in which it is postulated that for a highly sheared coronal magnetic field, the radiative (or condensation) time of the plasma is less than the free-fall time along the field, allowing an initial condensation, if its mass is sufficient, to deform the field slightly. This forms an equilibrium structure where a prominence could occur.

For frequencies greater than $\omega_{\text {crit }}$, the summit temperature "locks" into a quasisteady orbit about its steady state value. In Figure 4.11 the change in $T_{0}$ is plotted against $H$ for $\omega=50$ with the steady state $T_{0}$ values for constant $H_{0}$ 's shown by the dashed line. At time $t=t_{0}=0, T_{0}$ is at its equilibrium value. As $H(t)$ initially increases, $T_{0}$ rises and stays relatively close to the thermal equilibrium curve; at $t=t_{1}, H=100$, the maximum value of the heating. As the heating term decreases, $T_{0}$ falls and moves away from the equilibrium line - it reaches its minimum value just after $H\left(t_{2}\right)=0$. By the time $t=t_{3}, T_{0}$ has once again reached a value comparable to its steady state temperature but it has now been caught in a cyclic orbit. Thus, for this value of the frequency, the temperature is not evolving simply 


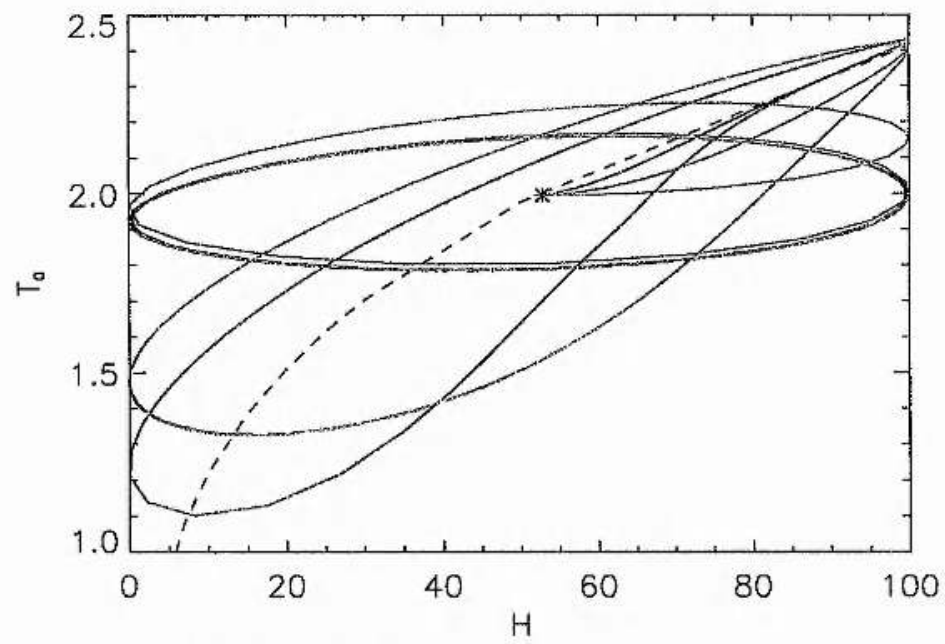

Figure 4.12: Variation of the summit temperature $T_{0}$ with heating function $H$ for $\omega=20$ (blue), $\omega=50$ (red) and $\omega=300$ (green).

through a set of static equilibria but follows quite a different scenerio away from equilibrium.

Figure 4.12 shows how the dynamic orbit changes as the frequency is altered. If $\omega$ is decreased to a value of 20 , then $T_{0}$ remains closer to the equilibrium curve as $H>H_{0}$ but it falls to a lower temperature for $H \sim 0$. If $\omega$ is increased to 300 then just as $T_{0}$ begins to rise, $H$ decreases again and therefore, after an initial overshoot, $T_{0}$ quickly settles into an elliptical type orbit centred on the equilibrium values. Increasing $\omega$ further simply flattens this final elliptical orbit.

Figure 4.13 shows the dependence of $\omega_{\text {crit }}$ upon the average heating value $H_{0}$ for a constant value of $b(=0.5) ; \omega_{\text {crit }}$ decreases with increasing $H_{0}$. If $H_{0} \rightarrow \infty$ then $\omega_{\text {crit }} \rightarrow 0$ as the loop would always be hot. If $H_{0}<\sim 3$ then $\omega_{\text {crit }} \rightarrow \infty$ as only cool solutions are possible.

Figure 4.14 shows the dependence of $\omega_{\text {crit }}$ upon $b$ for a constant value of $H_{0}$ $(=50.0)$; as the loop length increases, $\omega_{\text {crit }}$ gets larger. If $b<\sim 10^{-3}$ then only cool solutions exist and therefore $\omega_{\text {crit }}$ has a cut-off value.

With Figure 4.14 in mind, it is possible to model a nested set of coronal loops. As in Figure 3.2 in Section 3.1, we could have a set of thermally isolated fieldlines with the value of $b$ increasing outwards from the base along the nested sequence. If we assume that each fieldline receives the same amount of heat at the same frequency, then, if the outermost loop can be maintained at a hot solution, then the rest of the nested loops will also be hot. However, if the outermost loop cools, then so will all the rest of the loops in the structure. 


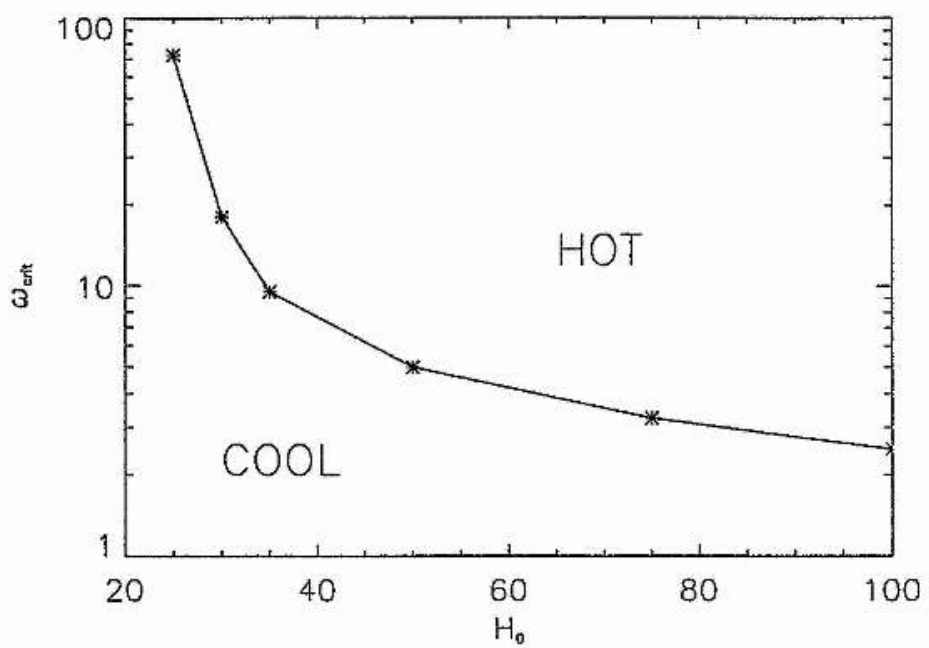

Figure 4.13: Dependence of $\omega_{\text {crit }}$ upon $H_{0}$ for $b=0.5$.

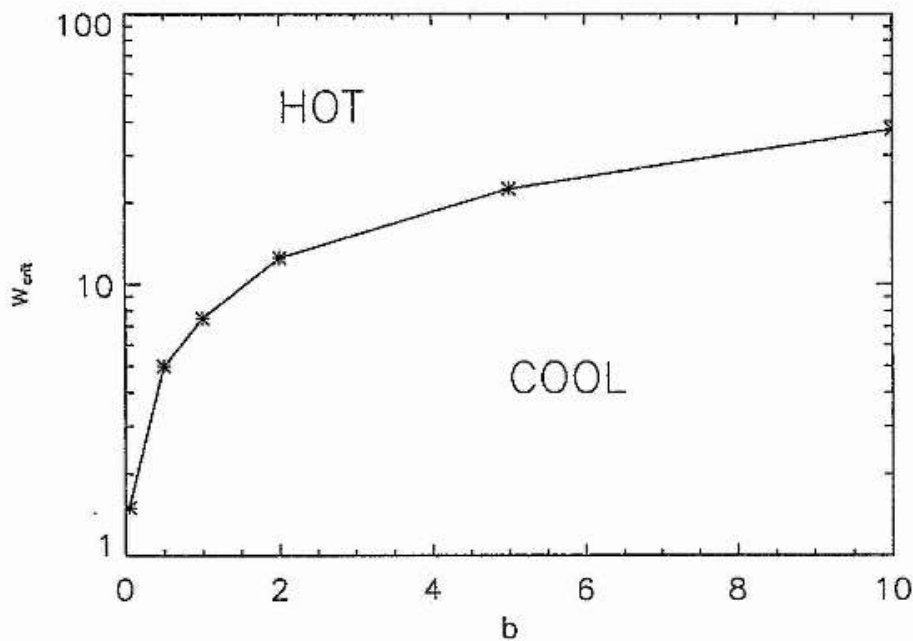

Figure 4.14: Dependence of $\omega_{\text {crit }}$ upon $b$ for $H_{0}=50$. 
Thus, it seems plausible that the corona must be heated on a regular basis in order to prevent the temperature from falling too far. In terms of our sinusoidal heating function (4.6), this means that $\omega$ must be sufficiently large to maintain a hot corona.

\subsection{Time-Dependent Heating of an Initially Static Loop including Hildner's Radiative Loss Function}

Since the Two Range Radiative Loss Function has all the important features of the full problem (outlined in Section 3.2), then the same physical effects will be occurring in both cases. Thus, all the conclusions that have been drawn from the simplified case will carry over to a more realistic form of the radiation. This can be shown by introducing the piecewise continuous fit of Hildner (1974) (see Table 4.4).

Following a similar approach as laid out in the Section 4.5, consider a similar static loop with the same value of the parameters $b, H_{0}$ and $T_{e}$. Using the heating

\begin{tabular}{|c|c|c|}
\hline$T$ & $\alpha$ & $\chi$ \\
\hline \hline$T<0.015$ & 7.4 & $2.24 \times 10^{13}$ \\
$0.015 \leq T<0.08$ & 1.8 & $1.37 \times 10^{3}$ \\
$0.08 \leq T<0.3$ & 0.0 & $1.45 \times 10^{1}$ \\
$0.3 \leq T<0.8$ & -2.5 & $7.15 \times 10^{-1}$ \\
$0.8 \leq T<10$ & -1.0 & 1 \\
\hline
\end{tabular}

Table 4.4: $\alpha$ and $\chi$ values (in non-dimensionalised form) for Optically Thin Radiation, obtained from analytical fit by Hildner (1974)

function (4.6), Figure 4.15 shows the summit temperature evolution for $\omega=5$ and 10. As in the simplified case, a critical value exists (in this case between 5 and 10) below which the plasma cools and above which the plasma remains hot and is of the order of (conductive timescale) ${ }^{-1}$, where $\tau_{\text {cond }} \approx 600 \mathrm{~s}$.

Airapetian and Smartt $(1994,1995)$ (see Section 4.2.4) reported that the repetition time calculated for coronal loop interactions from Yohkoh data was $\sim 200$ s. If we consider the smallest CLI energy release of $\sim 10^{26} \mathrm{ergs}$, then these impulsive events are occuring three times faster than the critical frequency calculated above. Therefore in this approximation, CLI's would be providing adequate energy, frequently enough to maintain a hot coronal loop structure.

Also, consider an Alfvén wave travelling along a resonant surface within a coronal 


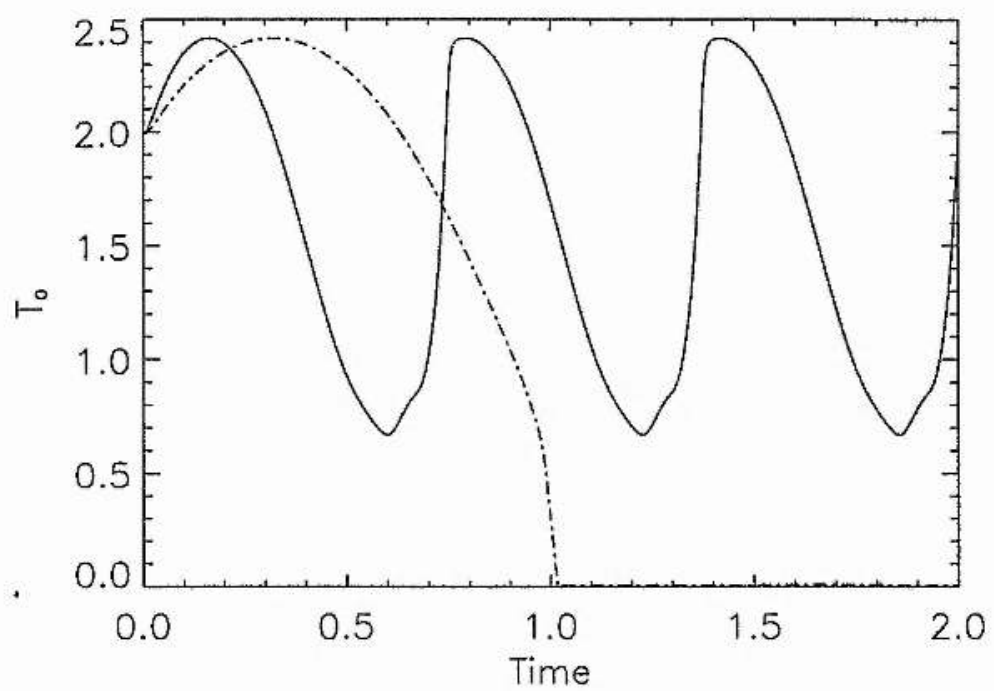

Figure 4.15: Evolution of the summit temperature $T_{0}$ with time for $\omega=5(-.--)$ and $10(-)$.

loop (see Section 4.2.1). A rough calculation of the Alfvén frequency of the wave could be

$$
\omega_{A} \approx \frac{2 \pi}{L} v_{A}
$$

where $L$ is the loop length, $v_{A}$ is the Alfvén speed in the corona $\left(\sim 1000 \mathrm{kms}^{-1}\right)$ and the loop is assumed to be semi-circular. For $L=60 \mathrm{Mm}, \omega_{A} \sim 0.01 \mathrm{~s}^{-1}$ compared with $\omega_{\text {crit }} \sim 0.001-0.002 \mathrm{~s}^{-1}$ above. Thus, wave heating of the loop cannot be ruled out on the grounds of the wave frequency. However, it must be noted that $v_{A}$ does depend on the overall magnetic structure as well as the density of the plasma in the loop.

\subsection{The Differential Emission Measure and Time-Dependent Heating}

\subsubsection{Background}

The principal source of information about the structure of the corona and transition region comes from observations of emission lines of UV to X-ray wavelengths in this optically thin environment (Athay, 1966). These observations are related to the plasma quantities through the differential emission measure which, for a simple 
planar geometry, can be defined as

$$
\operatorname{DEM}(T)=A n_{e}{ }^{2}\left|\frac{1}{T} \frac{d(\ln T)}{d s}\right|,
$$

where $A$ is the area of the region under investigation, $n_{e}$ is the electron number density and $d(\ln T) / d s$ is the temperature gradient along the line of sight. This is basically a measure of the amount of material that is present in the corona and transition region at a particular temperature.

The observations indicate that the $\operatorname{DEM}(T)$ has a deep minimum at $10^{5} \mathrm{~K}$ (Raymond and Doyle, 1981). Its shape appears to be universal across the Sun and shows little deviation from one solar region to another. It has also been observed for many late-type dwarfs (see Antiochos and Noci, 1986). The part of the DEM $(T)$ curve above $10^{5} \mathrm{~K}$ can be explained very well by a static hot-loop model. However the part below $10^{5} \mathrm{~K}$ cannot as there appears to be a greater amount of cooler material present in the corona than that calculated by a simple loop structure.

Several authors have put forward several different methods for explaining this discrepancy. Athay (1984) attributes the large emission at low temperatures to spicules. He considered an energy input that fluctuated both spatially and temporally such that the coronal atmosphere could be regarded as a mixture of coronal loops that are in equilibrium, cooling down or heating up. Athay argued that if the heating was switched off altogether, the loop temperature would fall, the plasma pressure would drop and material would flow out of the loop, the final result being a cool, evacuated flux tube. If the heating was switched on again rapidly, a massive upflow would occur - a jet of chromospheric gas moving up the flux tube preceded by a compression wave would be observed. The DEM profile for this scenario produced an increase below $10^{5} \mathrm{~K}$ due to the injection of cooler, denser material from below.

Rabin and Moore (1984) argued that although it has traditionally been regarded that the transition region is the thermal connection between the corona and the chromosphere, the lower transition region shows greater similarity to the upper chromosphere than the upper transition region and the corona. Thus, they treated the lower transition region as consisting of many low, closed loop structures rather than it existing primarily in the feet of coronal loops. Then the lower transition region would not be heated by energy transfer from above (by thermal conduction) but by ohmic heating of filamentary currents that flow along the magnetic field and therefore cause localised heating. Some of the currents may extend into the corona and therefore contribute to coronal heating but it was assumed that the majority of the current remained in small loops that had no coupling to the corona whatsoever. 
Again, calculations of the DEM profile showed an upturn below $10^{5} \mathrm{~K}$.

Antiochos and Noci (1986) argued that if the DEM above $10^{5} \mathrm{~K}$ can be explained by a hot coronal loop structure but below $10^{5} \mathrm{~K}$ needed a completely different mechanism, then it would seem feasible that in different solar regions at different times the relative strength of the two mechanisms would vary. Thus, the DEM should change - this is not the case. Either the two methods are very strongly linked or only one mechanism exists. Antiochos and Noci suggested that if hot coronal loop structures could explain the hot DEM part then the cool loop solutions of Hood and Priest (1979) could explain the cooler DEM part. Their model found that if both the hot and cool loops co-exist in the same region, the full form of the DEM could be reproduced.

Sturtock et al (1990) calculated the DEM curve created by a spicule-type model where the heating term was switched on episodically. The DEM profile matched the observed form though it must be noted that their model ignored the role of thermal conduction and plasma mass motions.

Steele and Priest (1990a) in their attempts to model the thermal equilibria along coronal loops found hot-cool solutions which had footpoints at $2 \times 10^{4} \mathrm{~K}$ and a similar summit temperature (or slightly lower) but with intermediate portions at coronal temperatures. They argued that these could explain the full DEM profile.

Mok and Van Hoven (1993) investigated the detailed structure of the magnetised transition region with a self-consistent 2D MHD numerical simulation. Initially the atmosphere had a checker-board-type horizontal structure (with cool peaks of chromospheric temperature extending up into the corona and hot valleys of coronal temperature extending down into the chromsphere) which was then allowed to relax. The authors found that although at high, coronal temperatures, the heat flux was predominantly parallel to the field, perpendicular conduction across the magnetic field became important below $10^{5} \mathrm{~K}$ and gave rise to the increased DEM profile below that temperature.

Van Hoven and Mok (1993) extended the above work to model a set of nested coronal loops, each loop being treated as an individual situation. The DEM curve was calculated as an average over all the loops and it was found that the lower transition region DEM depended purely on perpendicular conduction.

Cargill (1994a, 1994b) investigated the DEM of a model of a large scale coronal structure comprising of many small elemental flux tubes that are individually, impulsively heated. However, he was unable to reproduce an increasing DEM with decreasing temperature below $10^{5} \mathrm{~K}$. 


\subsubsection{Time-Dependent Differential Emission Measure}

It is possible to calculate the differential emission measure for the time-dependent evolution of the coronal loops considered in Section 4.5. Antiochos and Noci (1986) found that when they calculated the average emission measure for a set of nested, hot loops, the DEM produced resembled very closely the DEM curve for the hottest loop present. Thus, although a distribution of loops should be considered, we will simply investigate the DEM profile along a single fieldline that would be considered a. hot solution at its equilibrium values.

Since the cross-sectional area of the loop is constant, equation (4.12) can be written as,

$$
\operatorname{DEM}(T, t)=n_{e}{ }^{2}\left|\frac{1}{T(t)} \frac{d(\ln T(t))}{d x}\right| .
$$

where $x$ is the distance along the loop. Consider a $60 \mathrm{Mm}$ loop maintained by a heating frequency of $\omega=7.5$ as described in Section 4.5 and Figure 4.5. Figure $4.16 \mathrm{a}$ shows the evolution of the temperature along the loop for three separate times $\left(t_{1}<t_{2}<t_{3}\right)$ and Figure $4.16 \mathrm{~b}$ shows the coresponding DEM profiles.

At $t=t_{1}$, the temperature structure is basically the hot solution with the DEM showing that only hot material $\left(>10^{5} \mathrm{~K}\right)$ is present. At $t=t_{2}, H\left(t_{2}\right)<H\left(t_{1}\right)$ and $T(x)$ along the loop has decreased but when $t=t_{3}, H\left(t_{3}\right) \sim 0$ and a region of cool material has formed, extending in from the loop footpoint. The DEM profile for this time has a minimum at $\sim 10^{5.2} \mathrm{~K}$; there is an increase in the DEM below $10^{5} \mathrm{~K}$.

Thus the observed DEM (for example, Raymond and Doyle, 1981) can be explained in terms of a dynamic heating function rather than in the constant heating case. It must be noted that the rise in the DEM at low temperature occurs below $10^{4} \mathrm{~K}$. This is due to the fact that the values chosen for $T_{r}$ and $H_{0}$ are too small. If $T_{r}$ and $H_{0}$ were increased, the rise phase of the DEM curve would occur closer to that reported from observations. However, this does not negate the result that there is more cooler material present in the corona with this time-dependent model than with a simple, static model. Also, it has been argued that this DEM rise is only produced because the system is isobaric and therefore if the temperature falls the density must rise (Cargill, 1995, private communication).

\subsection{Conclusions}

Studies of the thermal structure of coronal loops assurne that the heating term is a given function of density, temperature and position but not a function of time. In this way the equilibrium structure of coronal loops can be determined. However, 


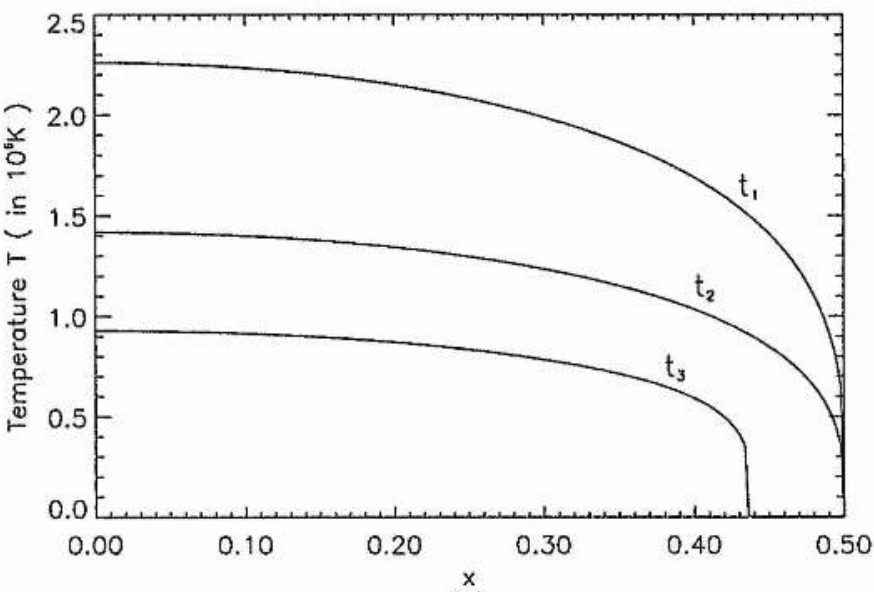

(a)

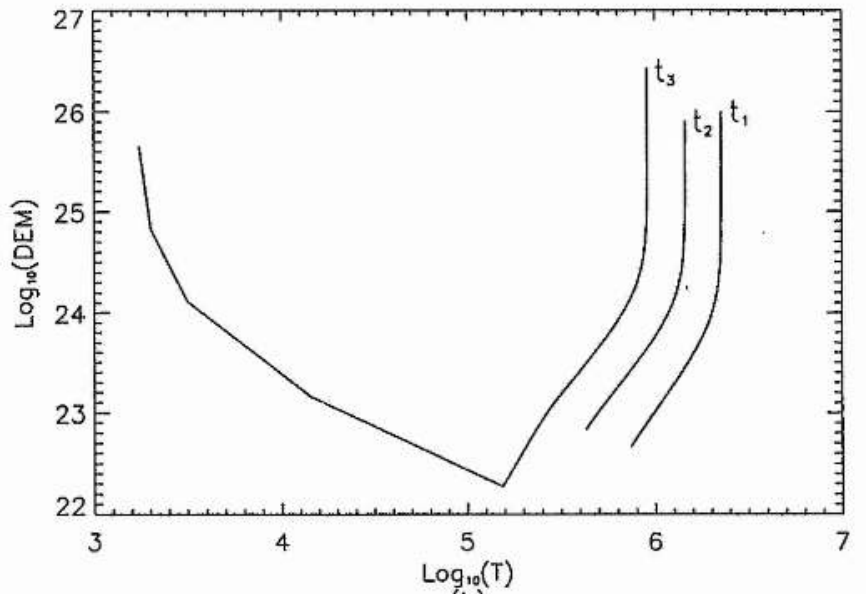

(b)

Figure 4.16: (a) Variation in the temperature along the loop (in units of $10^{6} \mathrm{~K}$ and (b) the corresponding differential emission measure profile for $\omega=7.5$. 
this assumption of constant heating is unrealistic since none of the present heating mechanisms can provide a continual, constant supply of heat; it will be intermittent or time-dependent.

In this chapter, we have investigated the response of a coronal loop to a timevarying supply of heat energy. The results show that if the heat is not supplied frequently enough, the plasma contained in the magnetic loop cools to chromospheric temperatures. Once cooled, increasing the heating frequency again does not return the plasma temperature to typical coronal values - the magnitude of the heating term needs to exceed a specific value to regain the hot loop solution. It was also found that the temperature along the loop does not simply step through a set if static equilibria but locks into a quasi-steady orbit around its equilibrium values.

As expected, the same results occur when the Hildner, (1974) estimates for the radiative losses are introduced. It was found in this case that the critical frequency was approximately of the order of (conductive timescale) $)^{-1}$, that is, a heating period of $\sim 600$ s.

The DEM was also investigated and for a heating frequency slightly above the critical value, there was an increase in the amount of cooler material $\left(\sim 10^{4} \mathrm{~K}\right)$ present in the corona than that calculated by simple, static models. 


\section{Chapter 5}

\section{Other Forms of Time-Dependent Heating}

Summer and winter, and springtime and harvest, Sun, Moon and stars in their courses above, Join with all nature in manifold witness, To thy great faithfulness, mercy and love.

(T.O. Chisholm, 1923)

In this chapter we investigate the coronal plasma response to other forms of timedependent heating namely pulse heating (sinusoidal, triangular and rectangular or "top-hat" patterns) and the release of random amounts of energy quanta of a fixed size over a fixed time interval.

\subsection{Sinusoidal Heating Pulse}

Consider a heating term of the form

$$
H(t)= \begin{cases}H_{0} \pi \sin \omega t & , H>0 \\ 0 & , H<0\end{cases}
$$

which has an average energy input per cycle of $H_{0}$. Using $H_{0}=50$ and $b=0.5$ as well as $T_{e}=0.1$ and $T_{r}=0.011$ in units of $10^{6} \mathrm{~K}$ to get the steady state temperature profile used in Section 4.5, Figure 5.1 shows the variation in the summit temperature $T_{0}$ as well as the heating pulses for $\omega=50$. Initially the temperature falls slightly as the heating pulse rises from zero. Then as $H$ exceeds $H_{0}$, the temperature increases but $T_{0}$ decreases again after the heat pulse has passed its maximum value. The summit temperature continues to fall when the heating is switched off 


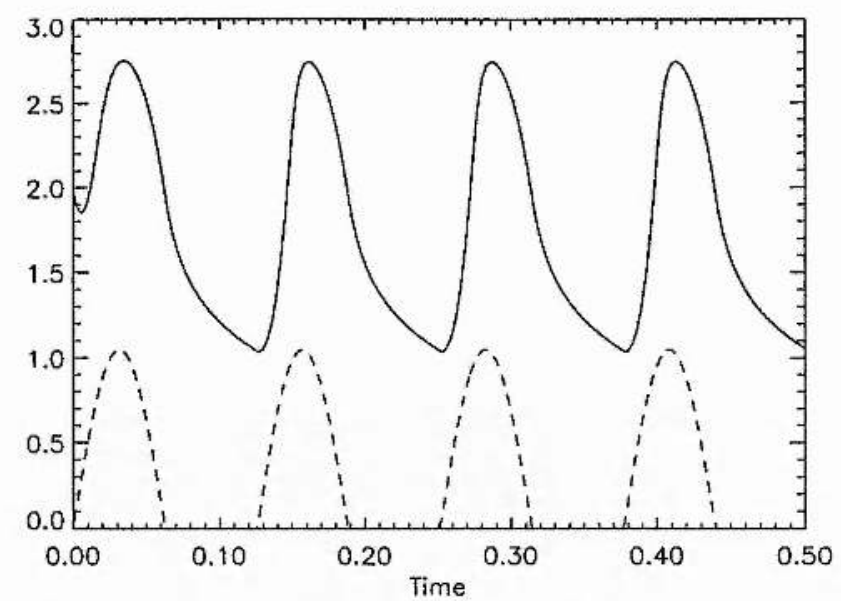

Figure 5.1: Variation in the summit temperature (-) with the corresponding heating term $H / 150$ (- - ) for $\omega=50$.

but rises dramatically as the next heat pulse arrives; a hot coronal loop structure is maintained and this cycle is repeated.

If the frequency is reduced to $\omega=20$ (Figure 5.2), the first pulse mimics the scenario above but in between the first and second heating bursts, $T_{0}$ has fallen too far that although the second pulse increases $T_{0}$ slightly, the loop temperature cannot recover coronal values and therefore cools. Thus a critical frequency exists which must be exceeded to maintain a hot loop solution (Figure 5.3).

\subsection{Triangular Heating Pulse}

Consider a heating scenario where the energy input is switched on for a time $\Delta_{t}$ and then off for $\Delta_{t}$. If the heat is switched on at time $t=n \Delta_{t}$ (where $n$ is an integer), then one form of heating could be

$$
H(t)= \begin{cases}\frac{8 H_{0}}{\Delta_{t}}\left(t-n \Delta_{t}\right) & , \text { for } n \Delta_{t} \leq t<(n+1 / 2) \Delta_{t} \\ \frac{8 H_{0}}{\Delta_{t}}\left((n+1) \Delta_{t}-t\right) & , \text { for } \quad(n+1 / 2) \Delta_{t} \leq t<(n+1) \Delta_{t} \\ 0 & , \text { for } \quad(n+1) \Delta_{t} \leq t<(n+2) \Delta_{t}\end{cases}
$$

the average heat deposition from $n \Delta_{t}$ to $(n+2) \Delta_{t}$ being $H_{0}$. Using the same initial temperature profile as in Section 5.1, Figure 5.4 shows the variation of $T_{0}$ with the corresponding heat pulses for $\Delta_{t}=0.1$. Initially $T_{0}$ falls as $H$ rises from zero. As $H$ reaches its maximum value, $T_{0}$ has increased by nearly $50 \%$ from its equilibrium value. The summit temperature falls as $H$ decreases but rises again as the next heating pulse arrives. This temperature variation is simply repeated. However, if 


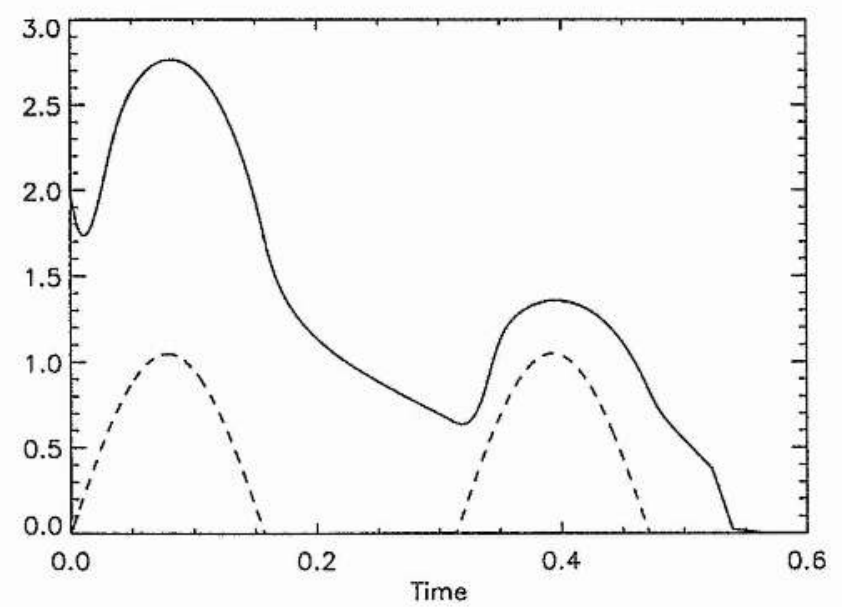

Figure 5.2: Variation in the summit temperature ( - ) with the corresponding heating term $H / 150$ $(--)$ for $\omega=20$.

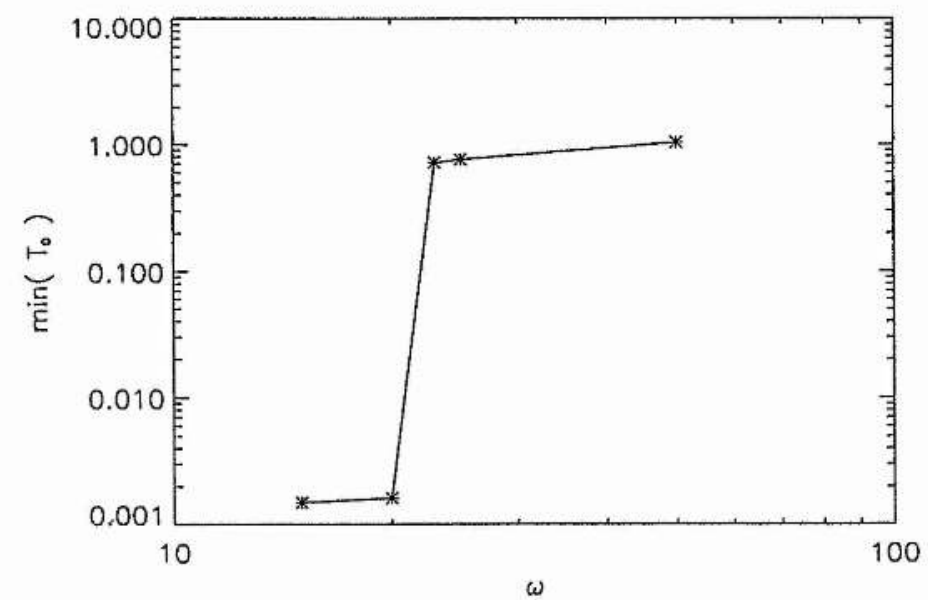

Figure 5.3: The minimum of the summit temperature against the frequency of the sinusoidal pulse heating function. 


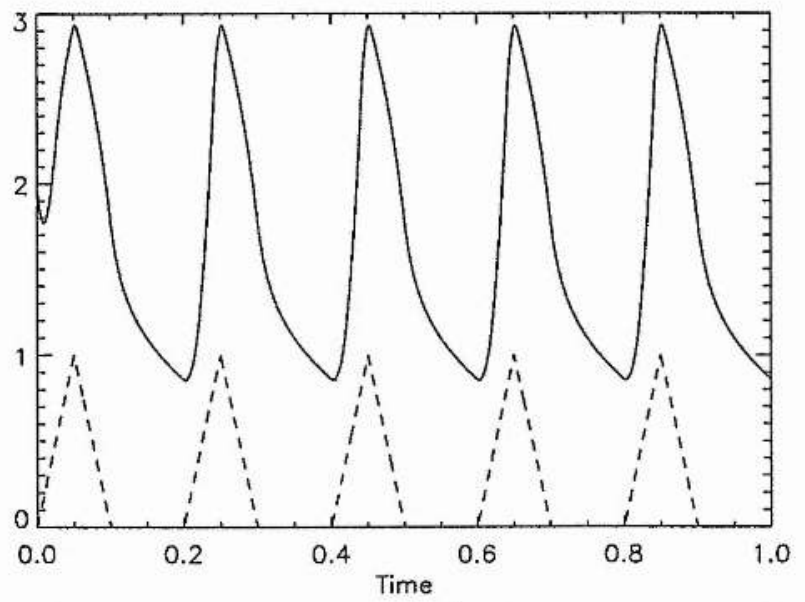

Figure 5.4: Variation in the summit temperature $(-)$ with the corresponding heating term $H / 200$ $(--)$ for $\Delta_{t}=0.1$.

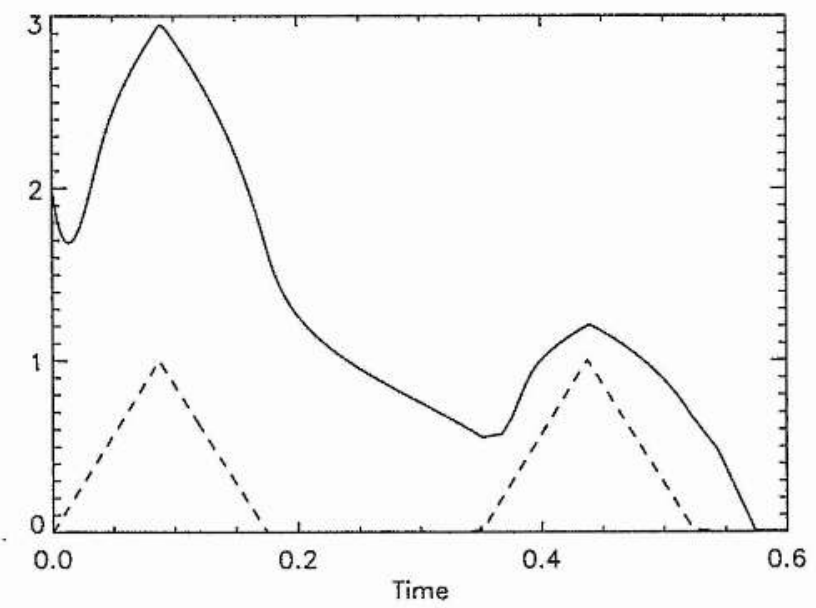

Figure 5.5: Variation in the summit temperature $(\rightarrow$ with the corresponding heating term $H / 200$ (- - -) for $\Delta_{t}=0.175$. 


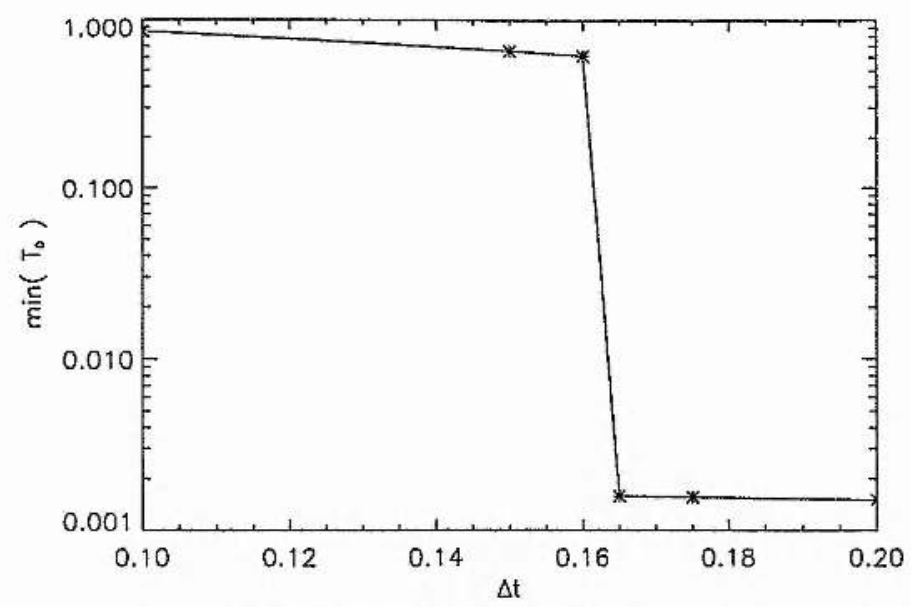

Figure 5.6: The minimum of the summit temperature against $\Delta_{t}$ for the triangular pulse heating function.

$\Delta_{t}=0.175$, a hot coronal loop solution cannot be maintained and a cool solution forms (Figure 5.5).

There is a critical timescale which must not be exceeded otherwise the loop temperature will cool along the length of the loop - this is shown in Figure 5.6.

\subsection{Rectangular Heating Pulse}

Another simple form of time-dependent heating is a rectangular or "top-hat" pulse function. Three cases are described below which depend on how long the pulse is switched on $\left(\Delta_{t o n}\right)$ and off $\left(\Delta_{t o f f}\right)$, each case beginning from the thermal equilibria set out in Section 5.1.

\subsection{1 $\Delta_{t \text { on }}=\Delta_{\text {toff }}$}

In this case, if $H$ is switched on at time $t=n \Delta_{t}$, then

$$
H(t)= \begin{cases}2 H_{0} & , \text { for } n \Delta_{t} \leq t<(n+1) \Delta_{t}, \\ 0 & , \text { for }(n+1) \Delta_{t} \leq t<(n+2) \Delta_{t}\end{cases}
$$

with average heating for the process being $H_{0}$. If we consider $\Delta_{t}=0.075$ (Figure 5.7), then $T_{0}$ initially rises and plateaus out as $H=100$ for the first $\Delta_{t}$ interval. Once the heating is switched off, $T_{0}$ cools to approximately half its original value before increasing again at the arrival of the second heating pulse. This heatingcooling cycle is repeated. 


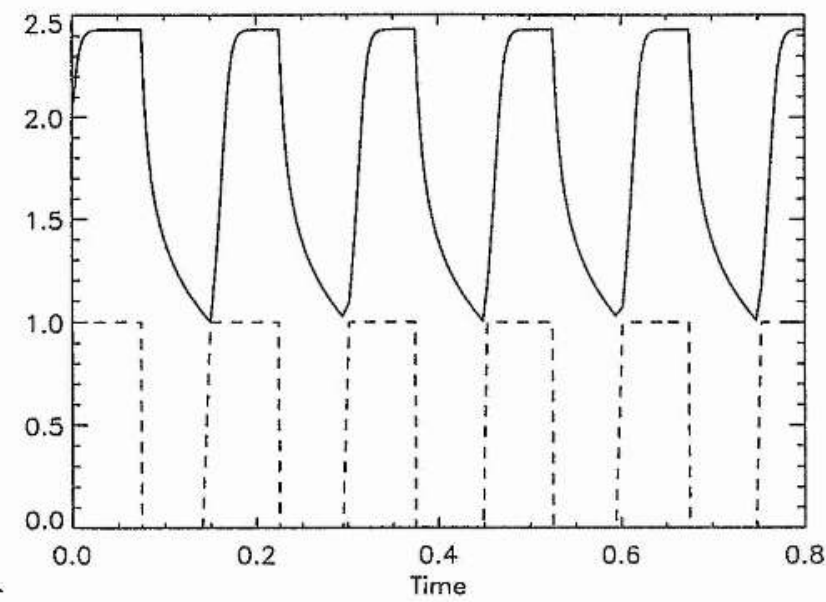

Figure 5.7: Variation in the summit temperature (-) with the corresponding heating term $H / 100$ $(--)$ for $\Delta_{t}=0.075$.

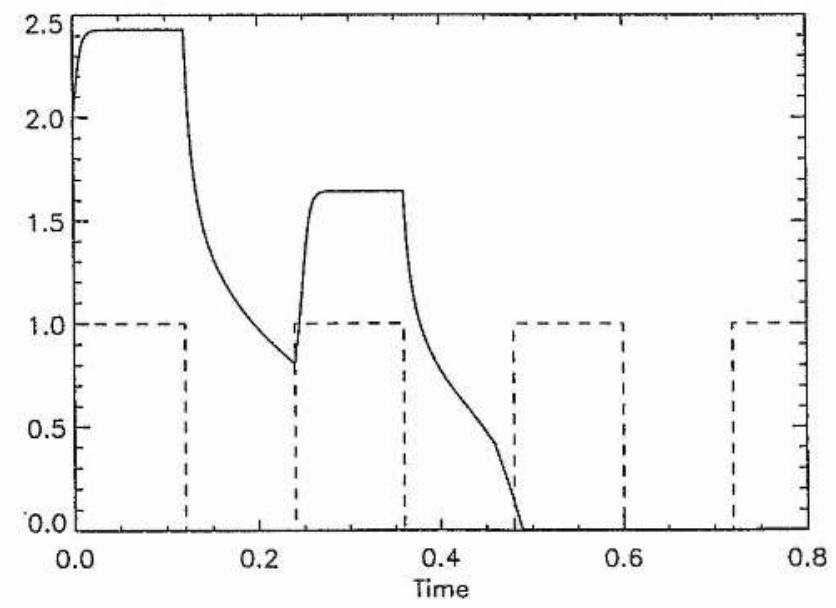

Figure 5.8: Variation in the summit temperature $(-)$ with the corresponding heating term $H / 100$ (- - ) for $\Delta_{t}=0.12$. 


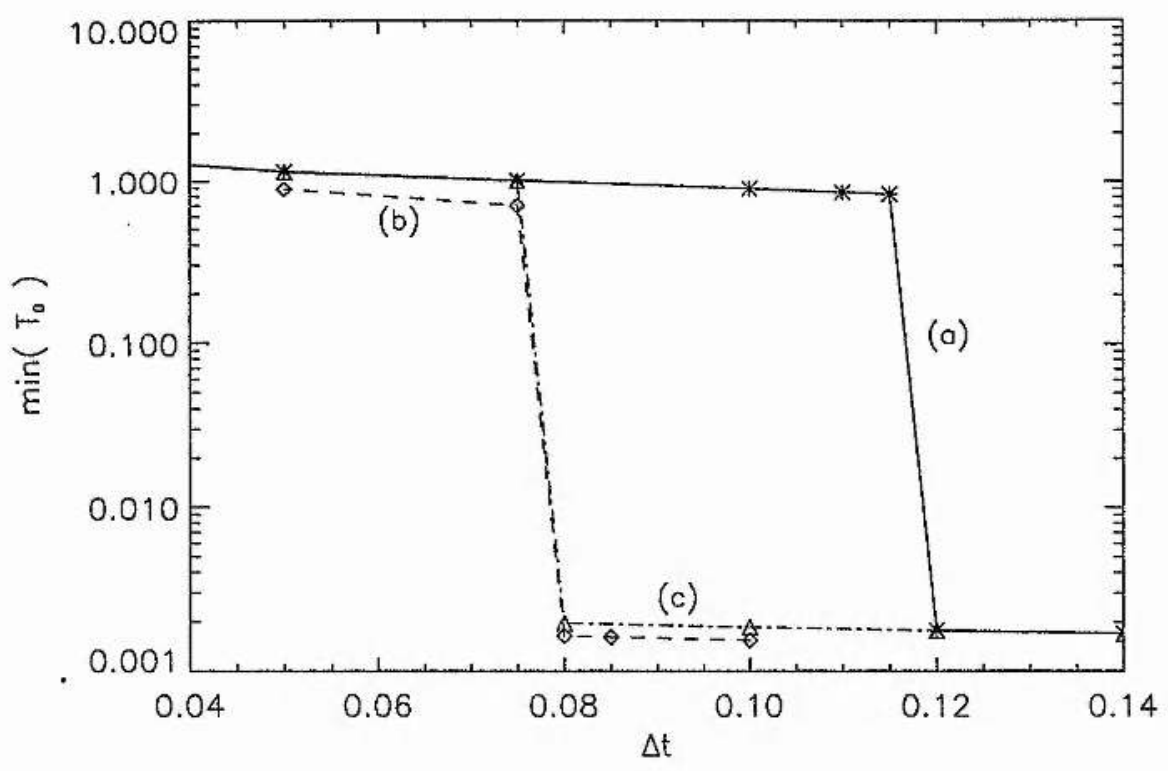

Figure 5.9: The minimum of the summit temperature against $\Delta_{t}$ for the rectangular pulse heating function where (a) $\Delta_{t \text { on }}=\Delta_{\text {toff }}(*) ;(\mathrm{b}) \Delta_{t \text { on }}=\Delta_{\text {toff }} / 2(\diamond)$ and (c) $\Delta_{t \text { on }}=2 \times \Delta_{\text {toff }}(\Delta)$.

Figure 5.8 shows what happens when $\Delta_{t}=0.12$. In the first cooling phase, $T_{0}$ falls to $\sim 0.8$ and although the second heating event raises the temperature again, the following cooling period allows the loop temperature time to cool. Figure 5.9(a) shows the minimum summit temperature against increasing values of $\Delta_{t}$ - once again, a critical timescale exists.

\subsection{2 $\Delta_{\text {ton }}=\Delta_{\text {toff }} / 2$}

If $H$ is switched on at time $t=n \Delta_{t}$, then

$$
H(t)= \begin{cases}3 H_{0} & , \text { for } n \Delta_{t} \leq t<(n+1) \Delta_{t} \\ 0 & , \text { for }(n+1) \Delta_{t} \leq t<(n+3) \Delta_{t},\end{cases}
$$

with average heating for the process being $H_{0}$. If $\Delta_{t}=0.05$ (Figure 5.10), $T_{0}(t)$ follows a similar pattern to that described in Section 5.3.1. With the heating initially switched on, $T_{0}$ rises and levels out; when the heating is switched off, $T_{0}$ cools but is able to recover with the energy input of the second heat pulse. If $\Delta_{t}=0.08$ (Figure 5.11), the loop temperature falls and remains cool after the second heating event. Thus a critical timescale exists which, if exceeded, allows the loop temperature to cool (see Figure 5.9(b)). 


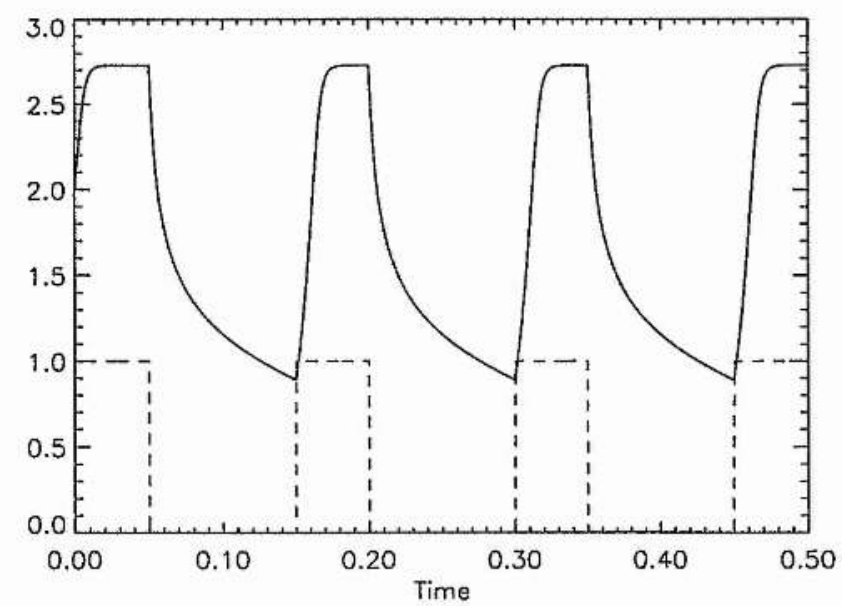

Figure 5.10: Variation in the summit temperature (-) with the corresponding heating term $H / 150$ $(--)$ for $\Delta_{t}=0.05$.

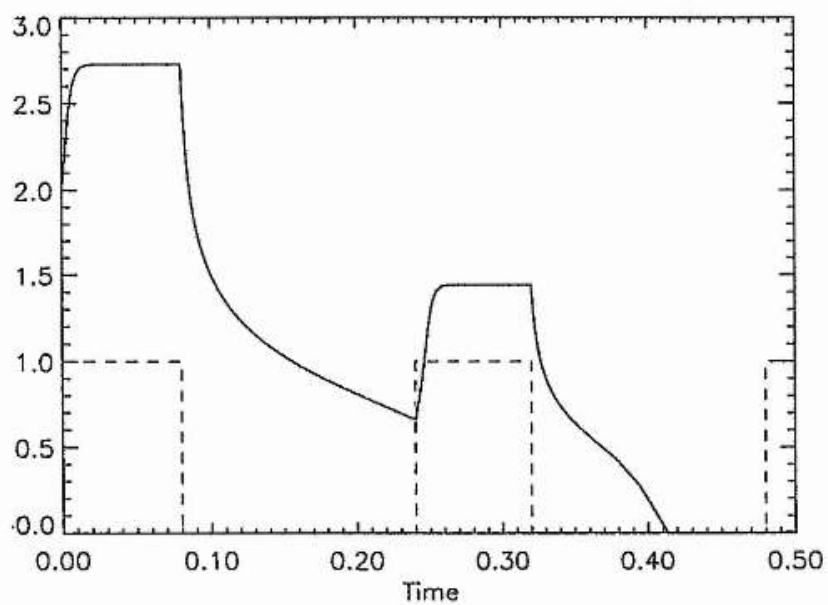

Figure 5.11: Variation in the summit temperature (-) with the corresponding heating term $H / 150$ $(--)$ for $\Delta_{t}=0.08$. 


\subsection{3 $\Delta_{\text {ton }}=2 \times \Delta_{\text {toff }}$}

If $H$ is switched on at time $t=n \Delta_{t}$, then

$$
H(t)= \begin{cases}\frac{3 H_{0}}{2} & , \text { for } n \Delta_{t} \leq t<(n+2) \Delta_{t}, \\ 0 & , \text { for }(n+2) \Delta_{t} \leq t<(n+3) \Delta_{t},\end{cases}
$$

with average heating for the process being $H_{0}$. The evolution of $T_{0}$ with respect to time is very similar to that described in Sections 5.3.1 and 5.3.2. Figure 5.9(c) shows that the critical timescale lies between 0.075 and 0.8 .

\subsubsection{Summary of Rectangular Pulse Heating}

Figure 5.9 compares the three different cases of this particular heating form for (a) $\Delta_{\text {ton }}=\Delta_{\text {toff }}$; (b) $\Delta_{t o n}=\Delta_{\text {toff }} / 2$ and (c) $\Delta_{\text {ton }}=2 \times \Delta_{t \text { off }}$. The critical timescales for (b) and (c) are nearly the same $\left(0.075<\Delta_{\text {tcrit }}<0.08\right)$, although (b) has a slightly lower $\min \left(T_{0}\right)$ than $(c)$. Thus although the rate of energy input per pulse for (b) $\left(=3 H_{0}\right)$ is twice that for $(\mathrm{c})\left(=3 H_{0} / 2\right)$, the former allows a longer time for the plasma to cool.

Note also that for $\Delta_{t}<0.075$ and $\Delta_{t}>0.12$, cases (a) and (c) are indistinguishable from each other.

\subsection{Random Heating Pulse}

Although it has been instructive to investigate the plasma response to a regular heat input, impulsive, bursty energy releases (such as microflares or nanoflares) requires process that is random in nature. There are many ways to model this scenario. As a first approximation, consider a heating supply where random amounts of a certain energy quanta, $\Delta_{H}$, are deposited in the loop in a fixed time interval $\Delta_{t}$. A Poisson process is often used to model the occurrence of events in time (Morgan, 1984) and it predicts that the probability $\left(P_{r}\right)$ of $k$ events occurring is

$$
P_{r}(k)=\frac{\mathrm{e}^{-\lambda} \lambda^{k}}{k !} \quad k=0,1, \ldots,
$$

where $\lambda$ is the average number of events per unit time. It is assumed that each energy deposition is independent from all the others. Thus in the time interval $n \Delta_{t}$, the heat released is

$$
H=k \Delta_{H}
$$




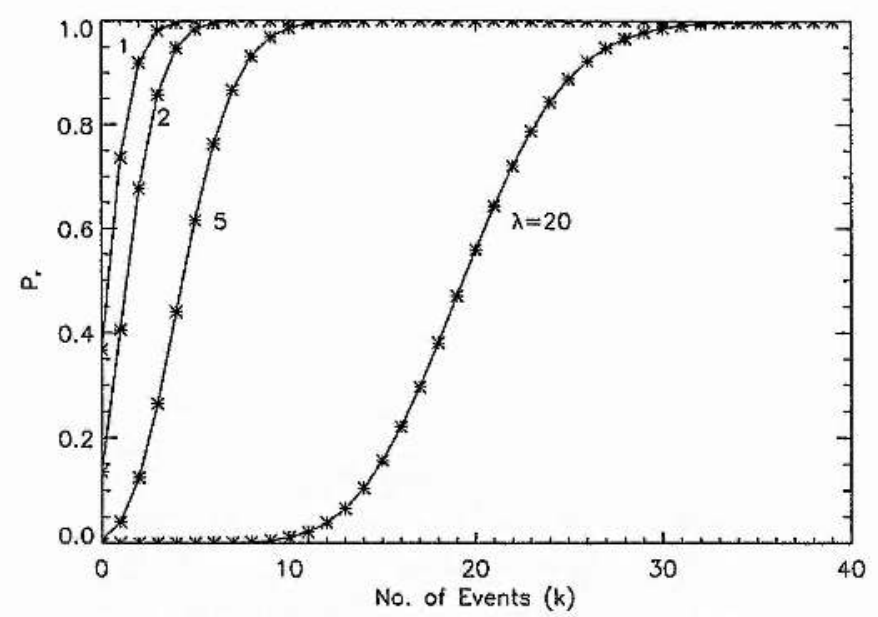

Figure 5.12: Probability Function $P_{r}$ against number of events for increasing $\lambda$.

where $k$ is generated from a random number $U$, uniform on $[0,1]$, such that

$$
\sum_{i=0}^{k} P_{i} \leq U<\sum_{i=0}^{k+1} P_{i}
$$

If we want the average heating value to be $H_{0}$, then

$$
\lambda=\frac{H_{0}}{\Delta_{H}}
$$

for a specified value of $\Delta_{H}$. There are two possible avenues of investigation.

\subsubsection{Fixed Time Interval}

Beginning with the thermal equilibrium profile considered in Sections $5.1-5.3$, consider the energy quanta (and their corresponding $\lambda$ ) shown in Table 5.1, released on a time interval of $\Delta_{t}=0.01(\sim 10 \mathrm{~s})$. Figure 5.12 shows the cumulative probability

\begin{tabular}{|c|c|c|}
\hline$\Delta_{H}$ & $\begin{array}{c}\sim \text { Energy Release } \\
\left(\text { ergs s}^{-1}\right)\end{array}$ & $\lambda$ \\
\hline 2.5 & $10^{24}$ & 20 \\
10.0 & $4 \times 10^{24}$ & 5 \\
25.0 & $10^{25}$ & 2 \\
50.0 & $2 \times 10^{25}$ & 1 \\
\hline
\end{tabular}

Table 5.1: Energy quanta and corresponding $\lambda$ values for the Poisson probability function.

against the number of events occurring for the range of $\lambda$ values from Table 5.1. 


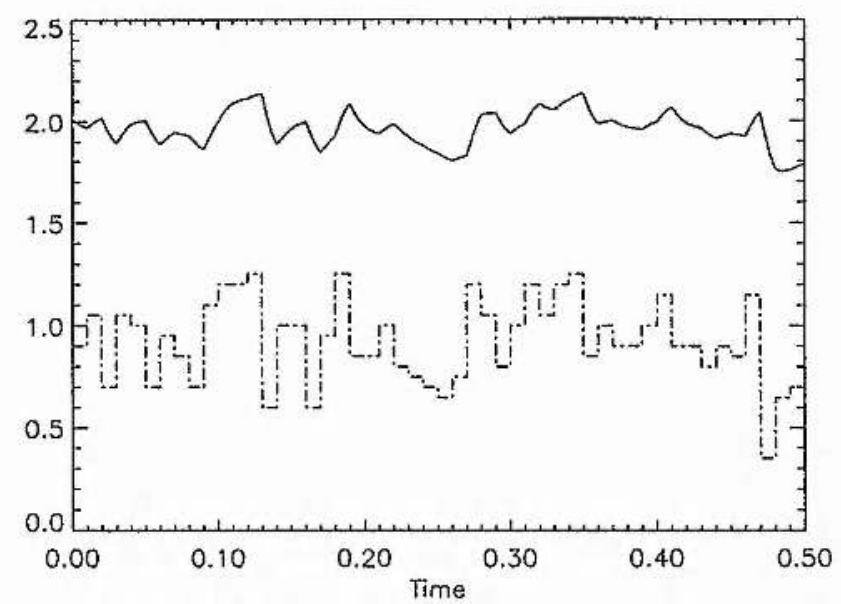

Figure 5.13: Variation in the summit temperature ( - ) with the corresponding heating term $H / 50$ $(--)$ for $\Delta_{H}=2.5$.

Figure 5.13 shows a typical random heating process for nanoflare-type energies $\left(\Delta_{H}=2.5\right)$. There is a very small probability of picking up a zero energy input and therefore it is likely that the loop will remain hot. For $\Delta_{H}=10$ (Figure 5.14), there is a greater variation in the summit temperature while for $\Delta_{H}=25,50$ (Figures 5.15 and 5.16), extended periods of no heating are possible and therefore the loop temperature has the opportunity to cool down.

Thus, with this particular Poisson model, nanoflare energy releases will keep the coronal plasma at typical coronal temperatures but energy bursts on the scale of microflares cannot (even on this short energy release time).

\subsubsection{Fixed Energy Quanta}

Consider a fixed amount of energy quanta, $\Delta_{H}=25.0(\lambda=2)$; from Section 5.4.1 we see that $T_{0}$ cools when $\Delta_{t}=0.01$.

Increasing $\Delta_{t}(=0.1$ in Figure 5.17) allows the plasma more time to cool and therefore $T_{0}$ falls to chromospheric temperatures faster. However, decreasing $\Delta_{t}$ $(=0.001$ in Figure 5.18) means that the plasma does not have time to respond to the rapid heat changes and therefore the temperature remains at coronal values.

\subsection{Conclusions}

In this chapter different forms of a time-dependent heat input were investigated. Heating a coronal loop by regular pulses (sinusoidal, triangular and "top-hat") all 


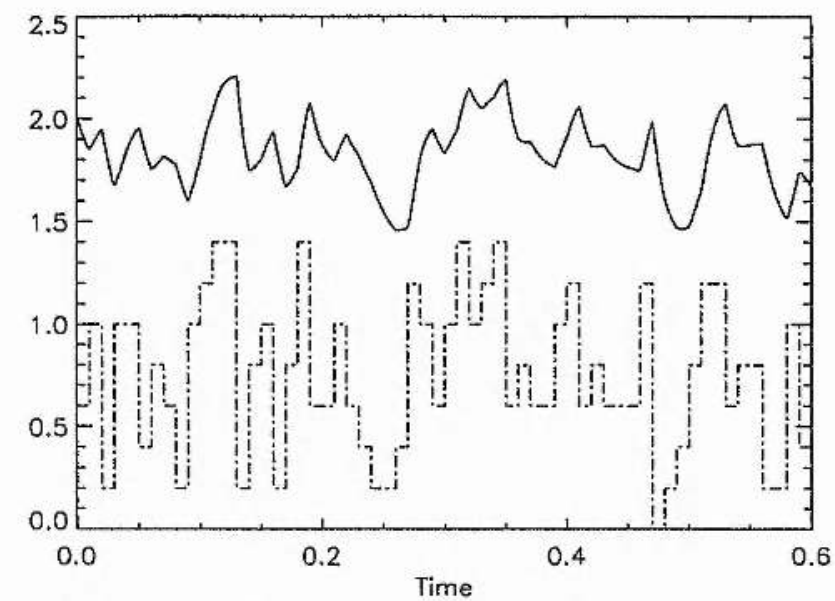

Figure 5.14: Variation in the summit temperature $(-$ ) with the corresponding heating term $H / 50$ $(--)$ for $\Delta_{H}=10.0$.

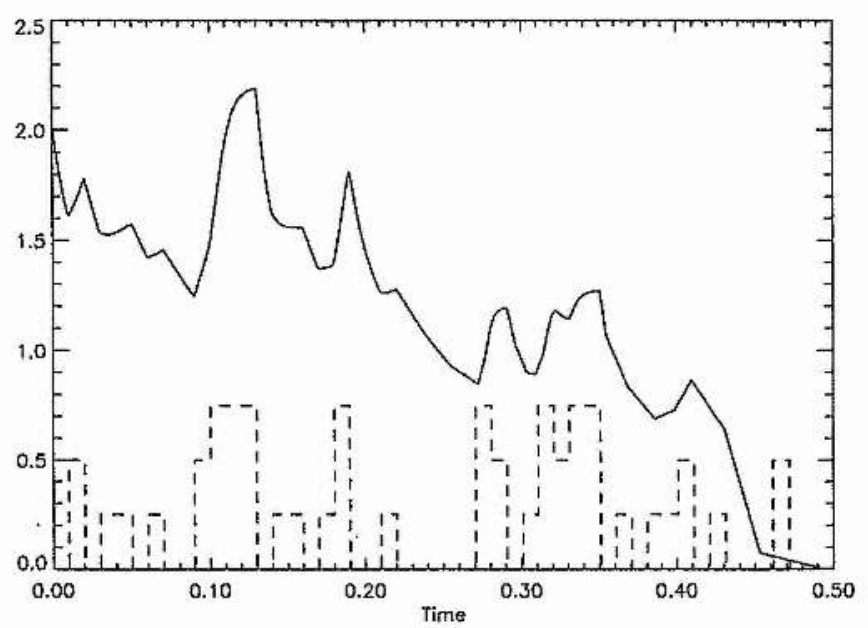

Figure 5.15: Variation in the summit temperature $(-)$ with the corresponding heating term $H / 100$ $(--)$ for $\Delta_{H}=25.0$. 


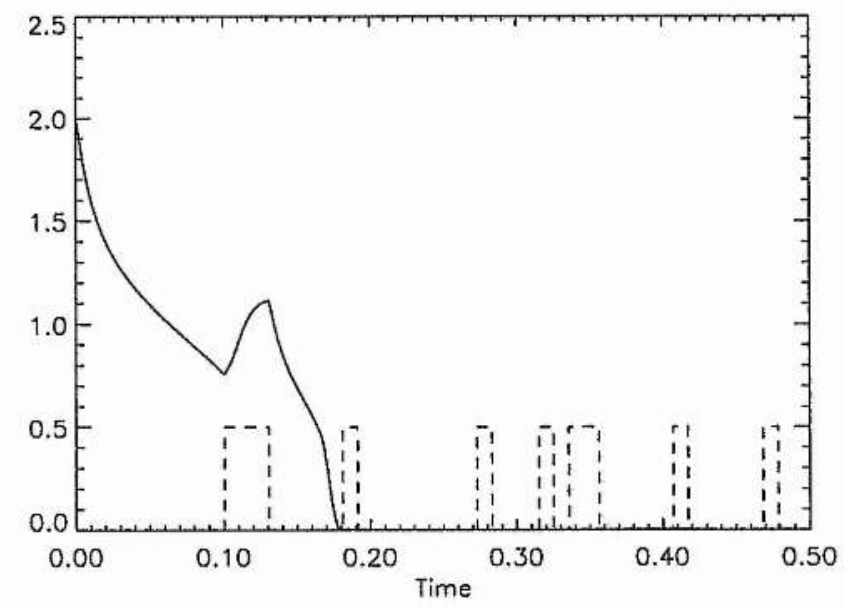

Figure 5.16: Variation in the summit temperature (-) with the corresponding heating term $H / 100$ $(--)$ for $\Delta_{H}=50.0$.

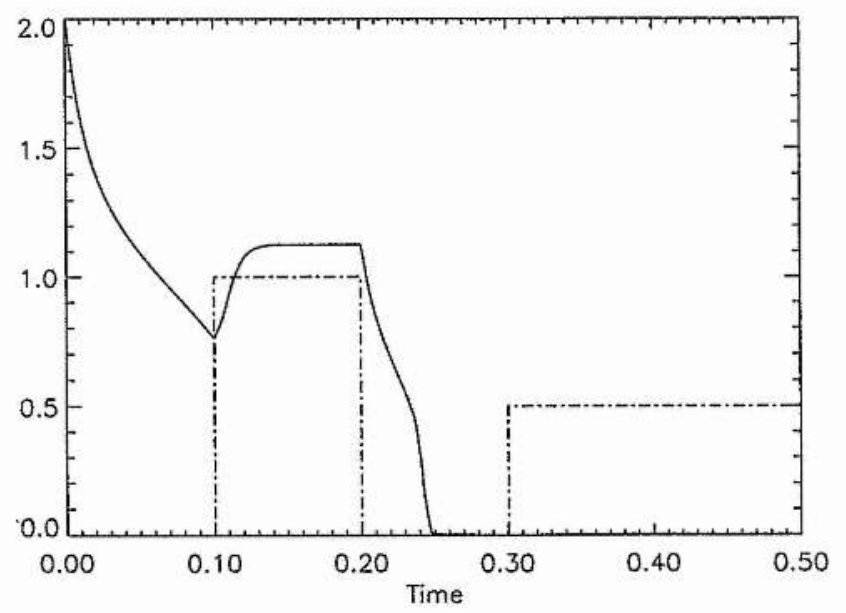

Figure 5.17: Variation in the summit temperature $(-)$ with the corresponding heating term $H / 50$ $(--)$ for $\Delta_{t}=0.1$. 


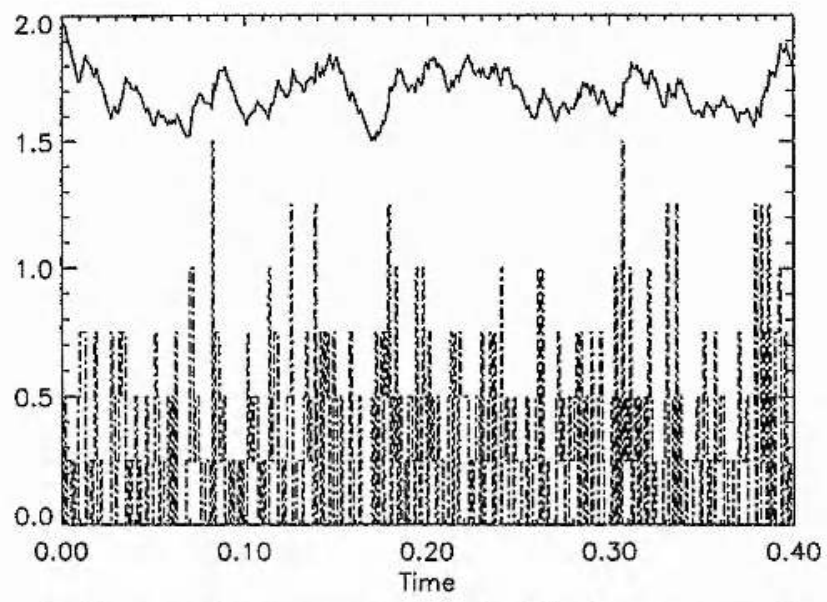

Figure 5,18: Variation in the summit temperature (-) with the corresponding heating term $H / 100$ $(--)$ for $\Delta_{t}=0.001$.

displayed a critical cooling timescale which, if exceeded, allows the loop temperature to cool to chromospheric values.

A Poisson process was introduced to model the occurrence of random energy release events in the corona and it was found that for fixed sizes of energy quanta over fixed time intervals, a hot loop solution can be maintained by nanoflare energy bursts over 10 s intervals while the more energetic microflares require their energy store to be released in the order of seconds to prevent the loop temperature from cooling. Possible extensions to random heating methods can be found in Chapter 7. 


\section{Chapter 6}

\section{Validity of the Isobaric Assumption to the Corona}

What has been will be again, What has been done will be done again;

There is nothing new under the Sun.

(Ecclesiastes 1:9)

\subsection{Introduction and Basic Equations}

The isobaric assumption introduced in Chapter 2 and used in all the investigations in Chapters 3 to 5 has had the computational convenience that the timesteps used in the numerical code are not restricted by the need to resolve adequately the sound waves travelling back and forth along the loop. Rather, the slower thermal variations can be followed with timesteps only restricted by the physical processes we wished to examine. However, it would be instructive to assess the validity of the isobaric scenario to the corona by including the inertial terms into the system. Thus from equations (2.23)-(2.26) we have

$$
\begin{aligned}
\frac{D \rho}{D t}+\rho \frac{\partial v}{\partial x} & =0 \\
\rho \frac{D v}{D t} & =-\frac{\partial p}{\partial x}+\rho g+\rho \nu \frac{\partial^{2} v}{\partial x^{2}} \\
\frac{\rho^{\gamma}}{\gamma-1} \frac{D}{D t}\left(\frac{p}{\rho^{\gamma}}\right) & =\kappa_{0} \frac{\partial}{\partial x}\left(T^{5 / 2} \frac{\partial T}{\partial x}\right)-\rho^{2} \chi T^{\alpha}+H \\
p & =\frac{R}{\tilde{\mu}} \rho T
\end{aligned}
$$


where all quantities are along the magnetic field as defined in Section 2.4. Using equations (6.1) to (6.4) in which gravity is neglected, the mass continuity equation (6.1) is

$$
\frac{\partial \rho}{\partial t}+\frac{\partial(\rho v)}{\partial x}=0
$$

The equation of motion (6.2) can be rewritten as

$$
\frac{D(\rho v)}{D t}-v \frac{D \rho}{D t}=-\frac{\partial p}{\partial x}+\rho \nu \frac{\partial^{2} v}{\partial x^{2}}
$$

and from equation (6.1),

$$
\frac{D(\rho v)}{D t}+\rho v \frac{\partial v}{\partial x}=-\frac{\partial p}{\partial x}+\rho \nu \frac{\partial^{2} v}{\partial x^{2}}
$$

or rather,

$$
\frac{\partial(\rho v)}{\partial t}+\frac{\partial\left(\rho v^{2}\right)}{\partial x}=-\frac{\partial p}{\partial x}+\rho \nu \frac{\partial^{2} v}{\partial x^{2}} .
$$

The left-hand side of the energy equation (6.3) can be expressed as

$$
\begin{aligned}
\frac{\rho^{\gamma}}{\gamma-1} \frac{D}{D t}\left(\frac{p}{\rho^{\gamma}}\right) & =\frac{R \rho^{\gamma}}{\tilde{\mu}(\gamma-1)} \frac{D}{D t}\left(\frac{T}{\rho^{\gamma-1}}\right) \quad \text { (from 6.4) } \\
& =\frac{R \rho}{\tilde{\mu}(\gamma-1)}\left(\frac{\partial T}{\partial t}+\frac{\partial(v T)}{\partial x}+(\gamma-2) T \frac{\partial v}{\partial x}\right),
\end{aligned}
$$

by expanding the total derivative with (6.1). Thus we have

$$
\frac{\partial T}{\partial t}+\frac{\partial(v T)}{\partial x}=\frac{\tilde{\mu}(\gamma-1)}{R \rho}\left[\kappa_{0} \frac{\partial}{\partial x}\left(T^{5 / 2} \frac{\partial T}{\partial x}\right)-\rho^{2} \chi T^{\alpha}+H\right]-(\gamma-2) T \frac{\partial v}{\partial x} .
$$

Equations (6.4)-(6.7) can be made dimensionless against typical coronal values. Following a similar analysis as in Section 2.5, set

$$
x=l \bar{x}, \quad t=t_{s} \bar{t}, \quad v=v_{s} \bar{v}=\frac{l \bar{v}}{t_{s}}, \quad T=T_{c} \bar{T}, \quad \rho=\rho_{c} \bar{\rho}, \quad p=p_{c} \bar{p},
$$

where $l$ is the length of the field line, $t_{s}$ is the sound travel timescale, $v_{s}=l / t_{s}$ is the acoustic velocity, $T_{c}=10^{6} \mathrm{~K}, \rho_{c}=8.35 \times 10^{-13} \mathrm{~kg} \mathrm{~m}^{-3}$ and $p_{c}=R \rho_{c} T_{c} / \tilde{\mu}$. Thus, we obtain

$$
\begin{aligned}
\frac{\partial \bar{\rho}}{\partial \bar{t}}+\frac{\partial(\overline{\rho v})}{\partial \bar{x}} & =0 \\
\frac{\partial(\overline{\rho v})}{\partial \bar{t}}+\frac{\partial\left(\bar{\rho}^{2}\right)}{\partial \bar{x}} & =-\frac{t_{s}^{2} p_{c}}{\rho_{c} l^{2}} \frac{\partial \bar{p}}{\partial \bar{x}}+\frac{t_{s} \nu}{l^{2}} \bar{\rho} \frac{\partial^{2} \bar{v}}{\partial \bar{x}^{2}},
\end{aligned}
$$




$$
\begin{aligned}
\frac{\partial \bar{T}}{\partial \bar{t}}+\frac{\partial(\bar{v} \bar{T})}{\partial \bar{x}} & =\frac{\epsilon_{0}}{\bar{\rho}}\left[\kappa_{0} \frac{\partial}{\partial x}\left(\bar{T}^{5 / 2} \frac{\partial \bar{T}}{\partial \bar{x}}\right)-b\left(\bar{p}^{2} \bar{\chi} \bar{T}^{\alpha-2}+\bar{H}\right)\right] \\
& -(\gamma-2) \bar{T} \frac{\partial \bar{v}}{\partial \bar{x}} \\
\bar{p} & =\bar{\rho} \bar{T}
\end{aligned}
$$

where we have introduced,

$$
H=H_{c} \bar{H}
$$

where $H_{c}=\rho_{c}{ }^{2} \chi_{c} T_{c}{ }^{\alpha_{c}}$ and $\chi_{c}$ and $\alpha_{c}$ are the values of the temperature dependent parameters when $T=\bar{T}_{c}$;

$$
b=\frac{\rho_{c}^{2} \chi_{c} T_{c}^{\alpha_{c}} l^{2}}{\kappa_{0} T_{c}^{7 / 2}}
$$

as the ratio of the conduction and radiative timescales;

$$
\epsilon_{0}=\frac{t_{s} \tilde{\mu}(\gamma-1) \kappa_{0} T_{c}^{5 / 2}}{R \rho_{c} l^{2}}
$$

as the ratio of the acoustic and conductive timescales and

$$
\bar{\chi}=\frac{\chi T_{c}^{\alpha}}{\chi_{c} T_{c}^{\alpha_{c}}},
$$

for each particular range of temperature for the piecewise fit of the optically thin radiation in the corona. If we consider

$$
\frac{t_{s}^{2} p_{c}}{\rho_{c} l^{2}} \equiv 1
$$

and define the sound speed squared as $c_{s}{ }^{2}=\gamma p_{c} / \rho_{c}$, then

$$
c_{s}{ }^{2}=\gamma v_{c}{ }^{2}
$$

which defines the sound timescale $t_{s}$. Also, if we shall represent the viscous term as

$$
\eta \frac{\partial^{2} \bar{v}}{\partial \bar{x}^{2}}
$$

where

$$
\eta \equiv \frac{t_{s} \nu}{l^{2}}
$$

and where the density dependence has been ignored for simplicity. With these particular parameter values, $\eta \sim 10^{-3}$; we shall inflate this value to $10^{-2} \leq \eta \leq 10^{-1}$ to help stabilise the numerical code. The final set of equations is

$$
\frac{\partial \rho}{\partial t}+\frac{\partial(\rho v)}{\partial x}=0
$$




$$
\begin{aligned}
\frac{\partial(\rho v)}{\partial t}+\frac{\partial\left(\rho v^{2}\right)}{\partial x} & =-\frac{\partial p}{\partial x}+\eta \frac{\partial^{2} v}{\partial x^{2}} \\
\frac{\partial T}{\partial t}+\frac{\partial(v T)}{\partial x} & =\frac{\epsilon_{0}}{\rho}\left[\frac{\partial}{\partial x}\left(T^{5 / 2} \frac{\partial T}{\partial x}\right)-b\left(p^{2} \chi T^{\alpha-2}+H\right)\right] \\
& -(\gamma-2) T \frac{\partial v}{\partial x} \\
p & =\rho T
\end{aligned}
$$

where all bars have been removed for convenience. To model a coronal loop structure, it is assumed that the loop is symmetrical $(0 \leq x \leq 0.5)$ and that the boundary conditions are

$$
\begin{aligned}
& \frac{\partial T}{\partial x}=\frac{\partial p}{\partial x}=\frac{\partial \rho}{\partial x}=0 \text { at } \quad x=0 \\
& T=T_{e}, \quad p=p_{e}, \quad \rho=\rho_{e}, \quad \text { at } \quad x=0.5
\end{aligned}
$$

where $T_{e}, p_{e}$ and $\rho_{e}$ is the chromospheric temperature, pressure and density respectively. The initial conditions are derived by setting all the time derivatives in (6.19)-(6.21) to zero and assuming that there are no plasma flows $(v=0)$. Thus, from $(6.20)$,

$$
\frac{\partial p}{\partial x}=0 \quad \text { which implies } \quad p(x, 0)=p_{e}
$$

and it follows that $T(x, 0)=T_{s}(x)$ where $T_{s}$ is the static temperature profile obtained for a constant value of the heating $\left(H=H_{0}\right)$ and is a solution of

$$
\frac{\partial}{\partial x}\left(T^{5 / 2} \frac{\partial T}{\partial x}\right)=b\left(p_{e}^{2} \chi T^{\alpha-2}+H_{0}\right)
$$

from equation (6.21).

\subsection{A Simplified Set of Equations}

In order to test the numerical algorithm (which can be found in Appendix G), the system of equations (6.19) - (6.22) were simplified to allow for an analytical investigation of the response of the various plasma properties to an initial perturbation. Thus, consider a simplified energy equation of the form

$$
\frac{\partial T}{\partial t}+\frac{\partial(v T)}{\partial x}=\frac{\epsilon_{0}}{\rho}\left[\frac{\partial^{2} T}{\partial x^{2}}-b(1-T)\right]-(\gamma-2) T \frac{\partial v}{\partial x}
$$

where there is no longer any $T^{5 / 2}$ dependence for the conduction and the radiation -heating term has been reduced to $(1-T)$ - this keeps the equation linear in $T$ but 
also retains the property that the radiation is greater than (less than) the heating when $T<1(T>1)$. The isothermal temperature profile $T(x)=1$ is now a steady state solution to the thermal equilibria of the above equations with $p(x)=1$ and therefore $\rho(x)=1$.

\subsubsection{Linearization of Equations}

It is possible to linearize equations $(6.19),(6.20),(6.22)$ and $(6.27)$ about the equilibrium values. Setting

$$
T=1+T_{1}, \quad p=1+p_{1}, \quad \rho=1+\rho_{1} \quad \text { and } \quad v=v_{1}
$$

where $T_{1}$, etc are small perturbed quantities, we get

$$
\begin{aligned}
\frac{\partial \rho_{1}}{\partial t}+\frac{\partial v_{1}}{\partial x} & \approx 0 \\
\frac{\partial v_{1}}{\partial t} & \approx-\frac{\partial p_{1}}{\partial x}+\eta \frac{\partial^{2} v_{1}}{\partial x^{2}} \\
\frac{\partial T_{1}}{\partial t}+(\gamma-1) \frac{\partial v_{1}}{\partial x} & \approx \epsilon_{0}\left[\frac{\partial^{2} T_{1}}{\partial x^{2}}+b T_{1}\right], \\
p_{1} & \approx \rho_{1}+T_{1} .
\end{aligned}
$$

Various harmonic oscillations can be introduced by setting

$$
f_{1}=f_{2} \mathrm{e}^{\sigma t} \cos k x
$$

for $T_{1}, p_{1}$ and $\rho_{1}$ which satisfies the boundary condition that the perturbations are zero at $x=1 / 2$, if $k=(2 n+1) \pi$ and $n$ is the mode number of the particular harmonic under investigation. For the velocity perturbation

$$
v_{1}=v_{2} \mathrm{e}^{\sigma t} \sin k x,
$$

which satisfies the boundary condition that $v_{1}=0$ at $x=0 . T_{2}$ etc... are the amplitudes of the perturbations and $\sigma$ is a decay rate. Substituting these into equations (6.28)-(6.30) gives

$$
\begin{aligned}
& \rho_{2}=-\frac{v_{2} k}{\sigma} \\
& p_{2}=\frac{\left(\sigma+k^{2} \eta\right) v_{2}}{k} \\
& T_{2}=-\frac{(\gamma-1) k v_{2}}{\left(\sigma+\epsilon_{0}\left(k^{2}-b\right)\right)}
\end{aligned}
$$


and therefore from (6.31),

$$
\frac{\sigma+k^{2} \eta}{k}=-\frac{k}{\sigma}-\frac{(\gamma-1) k}{\left(\sigma+\epsilon_{0}\left(k^{2}-b\right)\right)}
$$

or rather,

$$
\sigma^{3}+\left\{\epsilon_{0}\left(k^{2}-b\right)+k^{2} \eta\right\} \sigma^{2}+k^{2}\left\{\eta \epsilon_{0}\left(k^{2}-b\right)+\gamma\right\} \sigma+k^{2} \epsilon_{0}\left(k^{2}-b\right)=0
$$

which is the dispersion relation for the system. The general solution to (6.38) can be found using the mathematical package MAPLE (the MAPLE output can be found in Appendix D). We shall investigate this dispersion relation under different assumptions and compare the analytical results with those produced by the numerical code."

\subsubsection{Dependence of Dispersion Relation on $\epsilon_{0}$ and $b$}

To get some feel for the solutions of the dispersion relation, set $\gamma=2$ (to remove the $(\gamma-2) T \partial v / \partial x$ term ) and $\eta=0$ (no viscosity) so that (6.38) depends purely on $\epsilon_{0}$ and $b$. Thus, we now have,

$$
\sigma^{3}+\epsilon_{0}\left(k^{2}-b\right) \sigma^{2}+2 k^{2} \sigma+k^{2} \epsilon_{0}\left(k^{2}-b\right)=0 .
$$

It is possible to apply an asymptotic expansion in powers of $k$ to $(6.39)$ to get

$$
\begin{aligned}
\sigma_{1} & \approx-k^{2}+2 \\
\sigma_{2,3} & \approx-\frac{1}{2}\left(1+\frac{1}{k^{2}}\right) \pm i k\left(1+\frac{3}{8 k^{2}}\right),
\end{aligned}
$$

for $\epsilon_{0}=b=1$ - this is derived in Appendix E. There is one real root $\left(\sigma_{1}\right)$ and two complex conjugate roots $\left(\sigma_{2,3}\right)$ to the dispersion relation. $\sigma_{1}$ corresponds to the decay rate of the radiative mode while $\sigma_{2,3}$ correspond to the pressure waves moving across the system (to the left and right) with a decay rate of $\operatorname{Real}\left(\sigma_{2,3}\right)$ and with a frequency of Imaginary $\left(\sigma_{2,3}\right)$. Figure 6.1 shows the change in $\sigma_{1}$ and in the real and imaginary parts of $\sigma_{2,3}$ for increasing values of the mode number $n$. There is excellent agreement between the analytically calculated values $(-)$ and the asymptotic prediction $\left({ }^{*}\right)$. One interesting feature is that for these values of $\epsilon_{0}$ and $b$, mode numbers $n \geq 1$ have a longer decay time (smaller decay rate) than the fundamental mode. Thus, given any arbitrary disturbance, the fundamental oscillation will die away, leaving higher frequency oscillations behind. 

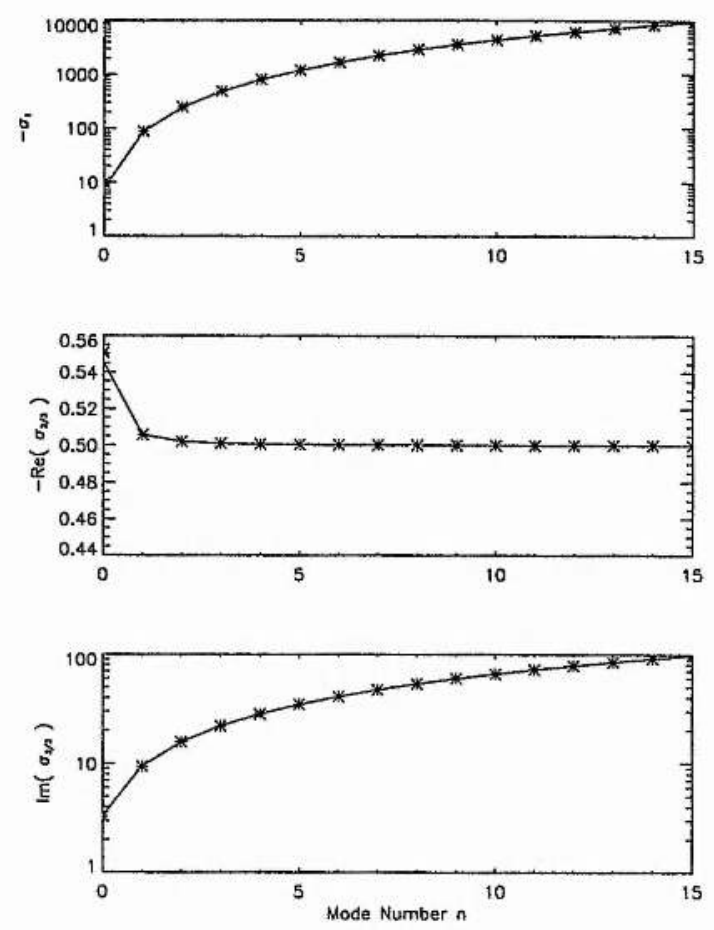

Figure 6.1: Analytical ( $\rightarrow$ ) and asymptotic prediction ( $\left.{ }^{* * *}\right)$ for the change in $\sigma_{1,2,3}$ with increasing mode number $n$ for $\epsilon_{0}=b=1$.

\section{Dependence on $b$}

Consider a fixed value of $\epsilon_{0}(=1)$ and let us concentrate upon the properties of the fundamental mode only $(n=0, k=\pi)$. Thus, equation (6.39) now reads as

$$
\sigma^{3}+\left(\pi^{2}-b\right) \sigma^{2}+2 \pi^{2} \sigma+\pi^{2}\left(\pi^{2}-b\right)=0 .
$$

- there is a change in the nature of the possible solutions when $b>\pi^{2}$. Figure 6.2 shows the increase in the decay rate of the fundamental radiative mode with increasing $b$. When $b$ exceeds $\pi^{2}$, this mode becomes unstable and any given perturbation grows. Figure 6.3 shows the dependence of $\operatorname{Re}\left(\sigma_{2,3}\right)$ on $b$. Again there is a change from stability to instability when $b>\pi^{2}$ while the imaginary part of $\sigma_{2,3}$ has a maximum value at $b=\pi^{2}$ (see Figure 6.4). Note that if $b$ gets very large then, from (6.42), we have

$$
-b \sigma^{2}-\pi^{2} b \approx 0
$$

and therefore $\sigma \approx \pm \mathrm{i} \pi$. Thus, $\operatorname{Re}\left(\sigma_{2,3}\right) \rightarrow 0$ and $\operatorname{Im}\left(\sigma_{2,3}\right) \rightarrow \pi-$ this is also shown in Figures 6.3 and 6.4 . 


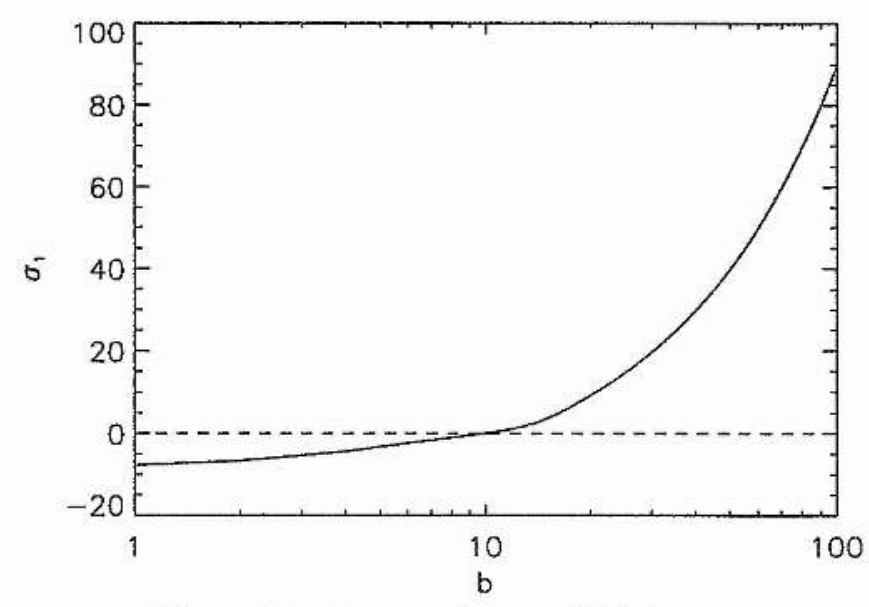

Figure 6.2: Increase in $\sigma_{1}$ with $b$ for $\epsilon_{0}=1$.

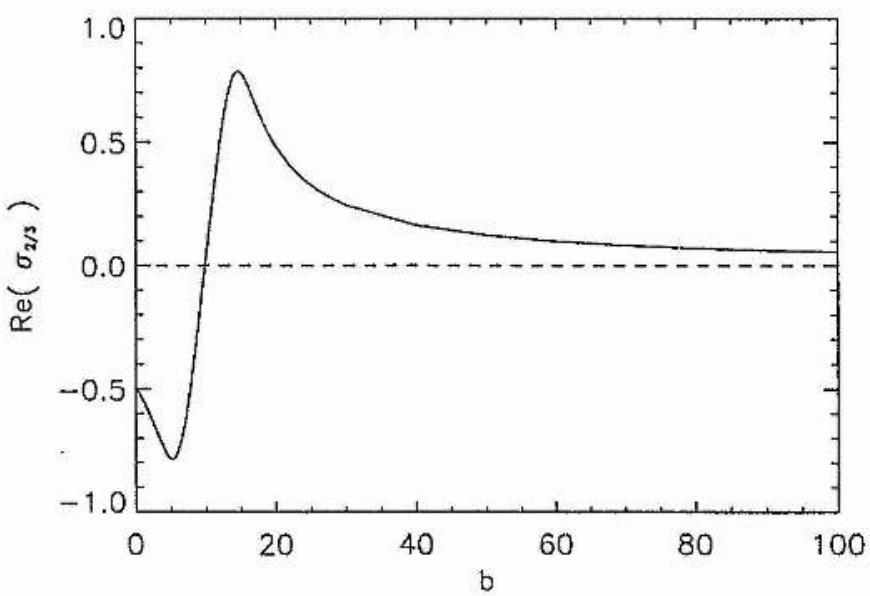

Figure 6.3: Increase in $\operatorname{Re}\left(\sigma_{2,3}\right)$ with $b$ for $\epsilon_{0}=1$. 


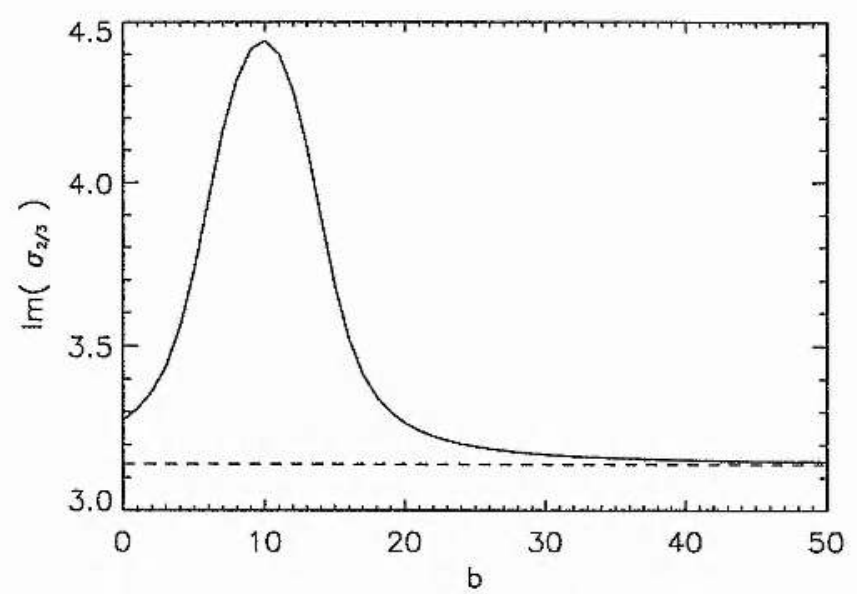

Figure 6.4: Increase in $\operatorname{Im}\left(\sigma_{2,3}\right)$ with $b$ for $\epsilon_{0}=1 ;(--)$ corresponds to $\operatorname{Im}\left(\sigma_{2 / 3}\right)=\pi$.

\section{Dependence on $\epsilon_{0}$}

Set $b=1$. Thus (6.39) can be written as,

$$
\sigma^{3}+\epsilon_{0}\left(k^{2}-1\right) \sigma^{2}+2 k^{2} \sigma+k^{2} \epsilon_{0}\left(k^{2}-1\right)=0 .
$$

As $\epsilon_{0}$ increases from zero, $\sigma_{1}$ always remains large and negative. If $\epsilon_{0} \ll 1$ then from $(6.43)$,

$$
\sigma^{3}+2 k^{2} \sigma \approx 0
$$

and therefore $\sigma \approx \pm \mathrm{i} \sqrt{2} k$. For $\epsilon \gg 1$,

$$
\epsilon_{0}\left(k^{2}-1\right) \sigma^{2}+k^{2} \varepsilon_{0}\left(k^{2}-1\right) \approx 0
$$

and thus $\sigma \sim \pm \mathrm{i} k$. Therefore the imaginary part of $\sigma_{2,3}$ simply varies between $\sqrt{2} k$ and $k$. Figure 6.5 shows the variation in the decay rates of the pressure mode $\left(\operatorname{Re}\left(\sigma_{2,3}\right)\right)$ for different harmonics $(n=0,1, \cdots, 10)$ with $\epsilon_{0}$. For $\epsilon_{0}>\sim 0.7$ the decay rate of the fundamental mode is larger than for the higher harmonics. However, if $\epsilon_{0}<\sim 0.7$, then the higher wave modes decay away faster than the fundamental.

\section{Dependence on $\epsilon_{0}$ and $b$}

Concentrating on the decay rate $\operatorname{Re}\left(\sigma_{2,3}\right)$ of the fundamental, Figure 6.6 shows a surface plot of how $\operatorname{Re}\left(\sigma_{2,3}\right)$ depends upon $b$ and $\epsilon_{0}$. Cross-sections of this plot for various values of $\epsilon_{0}$ are presented in Figure 6.7. As $\epsilon_{0}$ decreases from 1.0, the minimum and maximum on either side of $b=\pi^{2}$ are smoothed out. At $\epsilon_{0}=0.1$, they have disappeared altogether. If $\epsilon_{0}$ is decreased further, then, for $b<\pi^{2}$, the decay rate of the fundamental mode continues to decrease (that is, it takes a longer time to decay). 


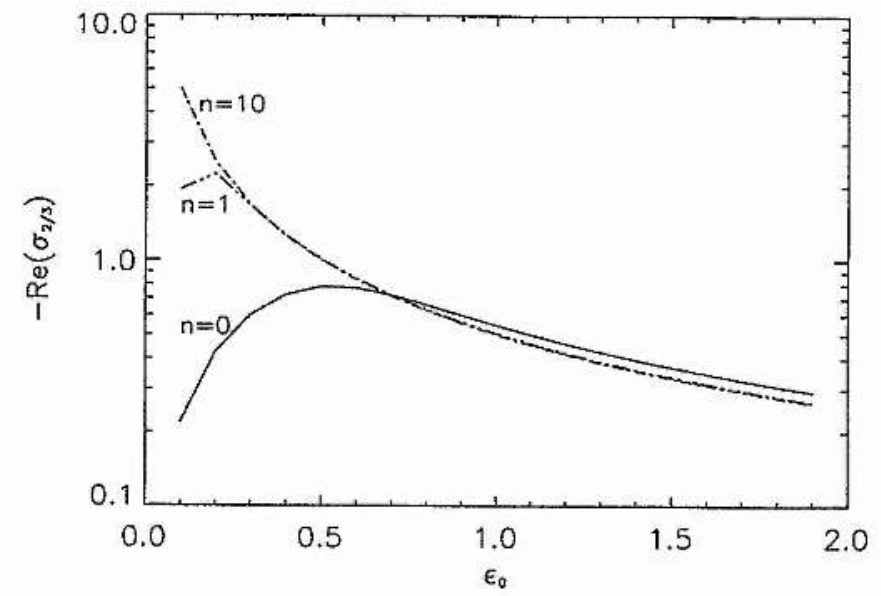

Figure 6.5: Variation in the decay rates of several harmonics with $\epsilon_{0}$ for $b=1$.

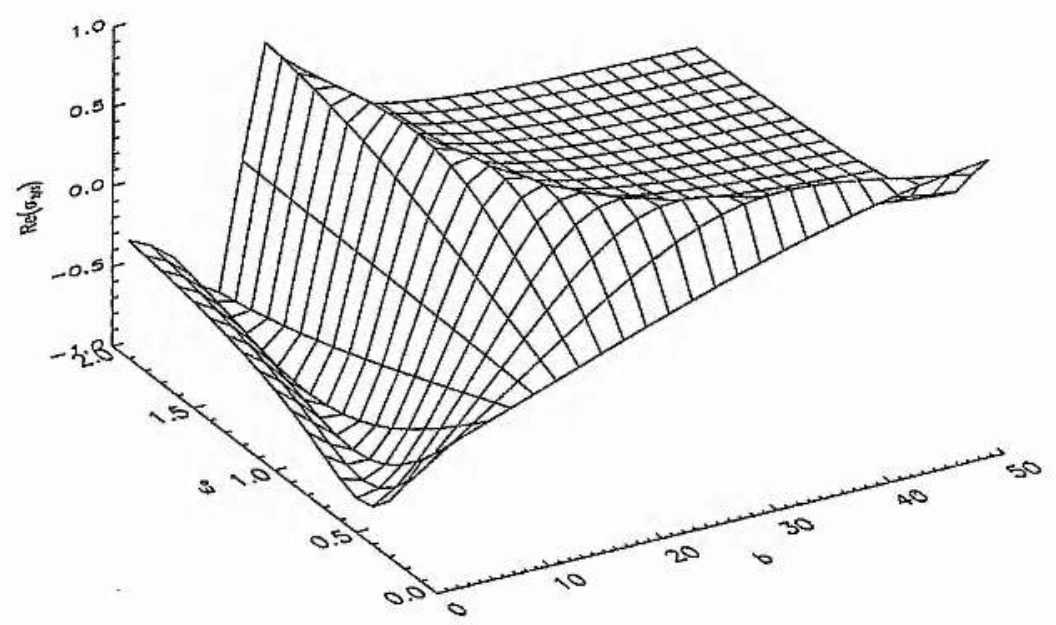

Figure 6.6: Surface plot for the dependence of the decay rate of the fundamental mode $\left(\operatorname{Re}\left(\sigma_{2,3}\right)\right)$ on $\epsilon_{0}$ and $b$. 


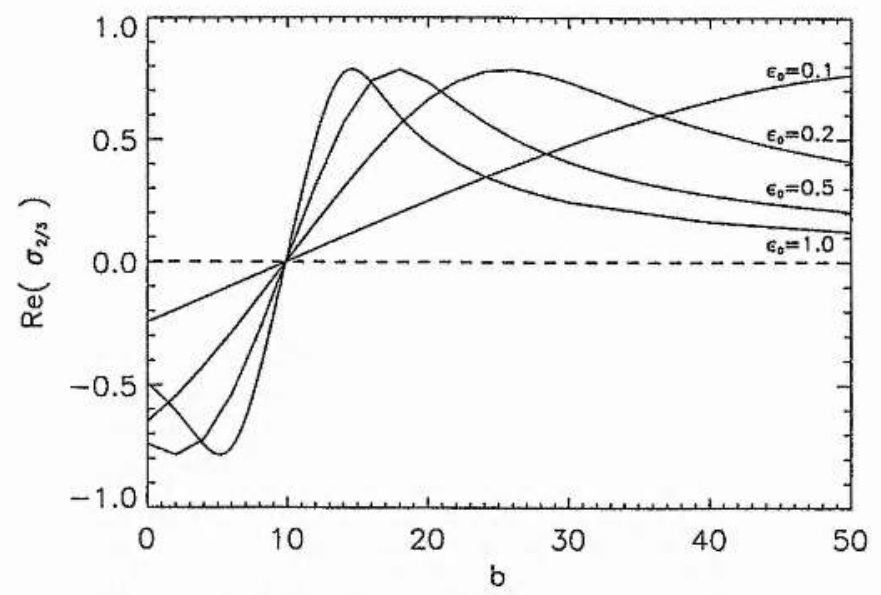

Figure 6.7: The dependence of the decay rate of the fundamental mode $\left(\operatorname{Re}\left(\sigma_{2,3}\right)\right)$ on $b$ for $\epsilon_{0}=$ $0.1,0.2,0.5,1.0$.

\subsubsection{Numerical Calculation for the Decay of an Initially Perturbed Uni- form Atmosphere}

The main reason for following this line of investigation is to derive analytical expressions for the time evolution of the temperature, density, pressure and velocity against which the results of the numerical algorithm can be compared. With this in mind, consider a fundamental perturbation $(n=0, k=\pi)$ to the isothermal atmosphere of the form

$$
T_{1}(x, t)=A \mathrm{e}^{\sigma_{r} t} \cos \left(\sigma_{i} t\right) \cos (\pi x)
$$

where $A$ is the initial amplitude of the perturbation with $\sigma_{r}=\operatorname{Re}\left(\sigma_{2,3}\right)$ and $\sigma_{i}=$ $\operatorname{Im}\left(\sigma_{2,3}\right)$ (whose values depend on $\epsilon_{0}$ and $b$ ). Thus from equation (6.30) we have,

$$
\frac{\partial T_{1}}{\partial t}+\frac{\partial v_{1}}{\partial x} \approx \epsilon_{0}\left[\frac{\partial^{2} T_{1}}{\partial x^{2}}+b T_{1}\right]
$$

and therefore substituting (6.44) into (6.45) gives

$$
v_{1}(x, t)=\frac{A \mathrm{e}^{\sigma_{r} t}}{\pi}\left[\left\{\left(b-\pi^{2}\right) \epsilon_{0}-\sigma_{r}\right\} \cos \left(\sigma_{i} t\right)+\sigma_{i} \sin \left(\sigma_{i} t\right)\right] \sin (\pi x) .
$$

From equations (6.29) and (6.46) we get,

$$
\begin{array}{r}
p_{1}(x, t)=\frac{A \mathrm{e}^{\sigma_{r} t}}{\pi^{2}}\left[\left\{\sigma_{r}\left(\left(b-\pi^{2}\right) \epsilon_{0}-\sigma_{r}\right)+\sigma_{i}^{2}\right\} \cos \left(\sigma_{i} t\right)\right. \\
\left.-\left\{\left(b-\pi^{2}\right) \epsilon_{0}-2 \sigma_{r}\right\} \sigma_{i} \sin \left(\sigma_{i} t\right)\right] \cos (\pi x),
\end{array}
$$


and from (6.31),

$$
\rho_{1}=p_{1}-T_{1} .
$$

The values of $\sigma_{r}$ and $\sigma_{i}$ are calculated from the analytical solution of the dispersion relation (6.38) while the numerical code is simply given the initial conditions (at $t=0)$,

$$
\begin{aligned}
T(x, 0) & =1+A \cos (\pi x), \\
v(x, 0) & =\frac{A}{\pi}\left\{\left(b-\pi^{2}\right) \epsilon_{0}-\sigma_{r}\right\} \sin (\pi x), \\
p(x, 0) & =1+\frac{A}{\pi^{2}}\left[\sigma_{r}\left(\left(b-\pi^{2}\right) \epsilon_{0}-\sigma_{r}\right)+\sigma_{i}{ }^{2}\right] \cos (\pi x), \\
\rho(x, 0) & =\frac{p(x, 0)}{T(x, 0)}
\end{aligned}
$$

and allowed to relax. As an example, set $\epsilon_{0}=1$ and $b=5$; for these parameter values, $\sigma_{1}$ is large and negative and has a very small contribution to the overall evolution of the system. It should be noted that from Figure 6.5, the value of $\epsilon_{0}$ chosen means that the decay time of the harmonics is longer than for the fundamental. However, since we are giving the system an initial fundamental perturbation, no other harmonics should appear and the system will be dominated by the decay of the fundamental mode. Figure 6.8 shows the relaxation of the temperature, pressure and density (at $x=0$ ) and the velocity (at $x=1 / 2$ ) from their initially maximum perturbed values ( $A=0.1$ in this case). There is very good agreement between the analytical $\left({ }^{*}\right)$ and numerical $(-)$ results.

\subsubsection{Addition of Viscosity}

Consider the dispersion relation (6.38) now with $\gamma=5 / 3$ and the viscosity term included. Figure 6.9 shows the variation in the decay rate $\operatorname{Re}\left(\sigma_{2,3}\right)$ for different mode numbers for increasing values of $\epsilon_{0}$ with $b=1$ and $\eta=0.01$. In contrast with Figure 6.5 , we see that the addition of a small amount of viscosity alters $\operatorname{Re}\left(\sigma_{2,3}\right)$ such that its value for all harmonics $n>0$ is greater than for the fundamental. Thus, given an arbitrary disturbance, all higher harmonics will decay away, leaving the fundamental oscillation behind.

As in Section 6.2.3, if the initially uniform atmosphere is given a fundamental perturbation of the form shown in equation (6.44), then corresponding evolutionary equations for the other perturbed quantities are

$$
v_{1}(x, t)=\frac{A \mathrm{e}^{\sigma_{r} t}}{(\gamma-1) \pi}\left[\left\{\left(b-\pi^{2}\right) \epsilon_{0}-\sigma_{r}\right\} \cos \left(\sigma_{i} t\right)+\sigma_{i} \sin \left(\sigma_{i} t\right)\right] \sin (\pi x),
$$



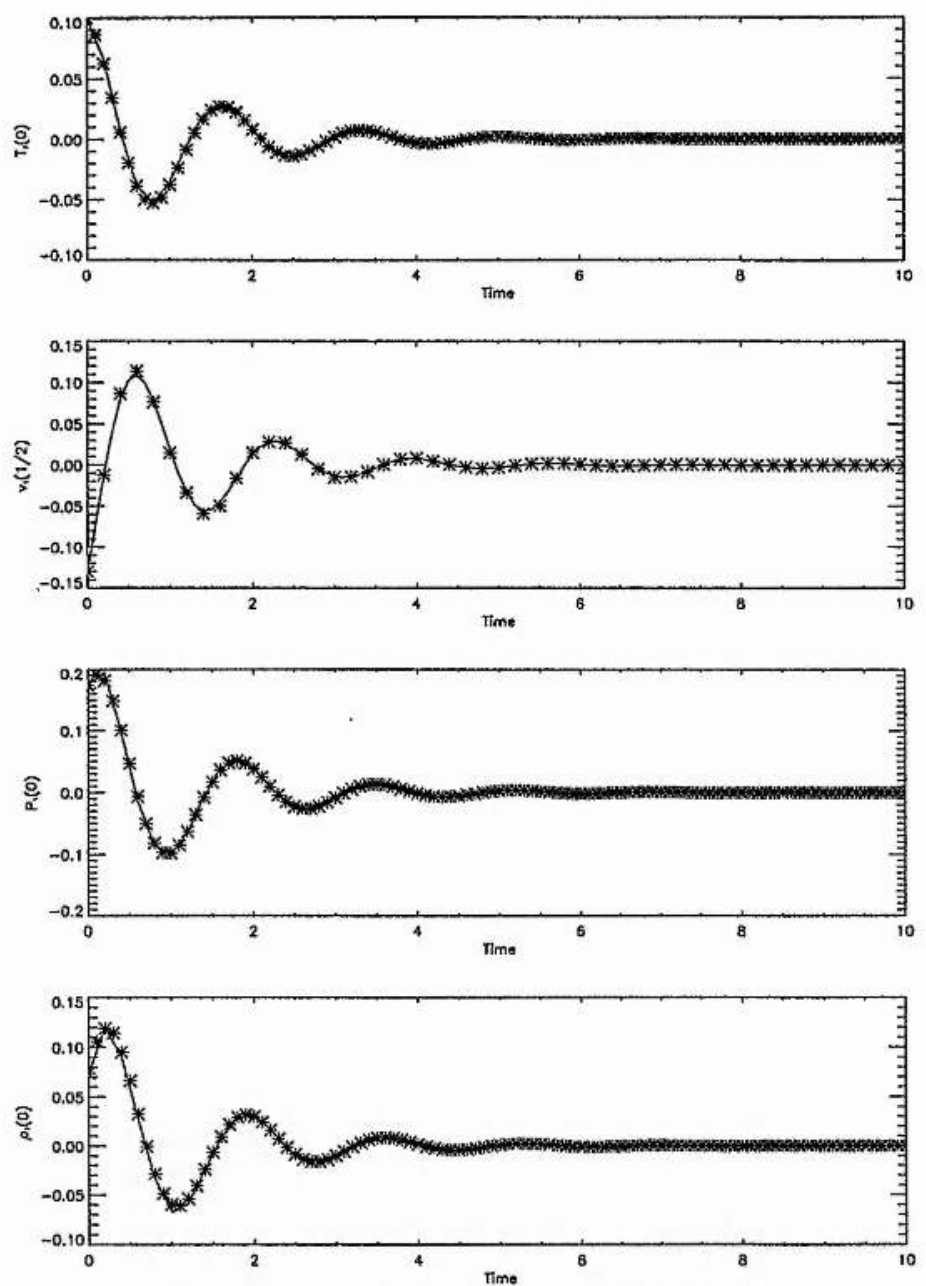

Figure 6.8: Analytical (***) and numerical calculations (-) for the relaxation of the temperature $\left(T_{1}\right)$, velocity $\left(v_{1}\right)$, pressure $\left(p_{1}\right)$ and density $\left(\rho_{1}\right)$ perturbations for $\epsilon_{0}=1, b=5$ and $A=0.1$.

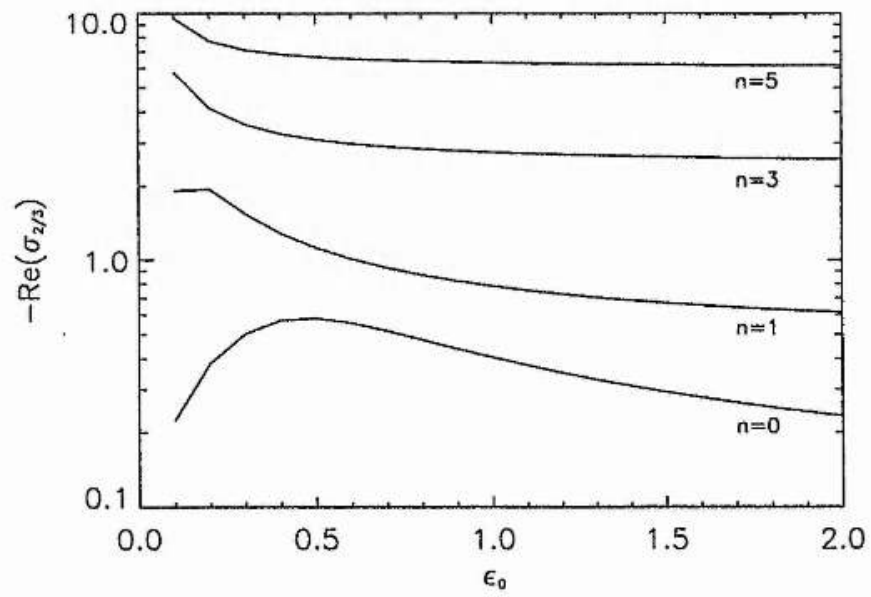

Figure 6.9: Variation in the decay rates of several harmonics with $\epsilon_{0}$ for $b=1$ and $\eta=0.01$. 

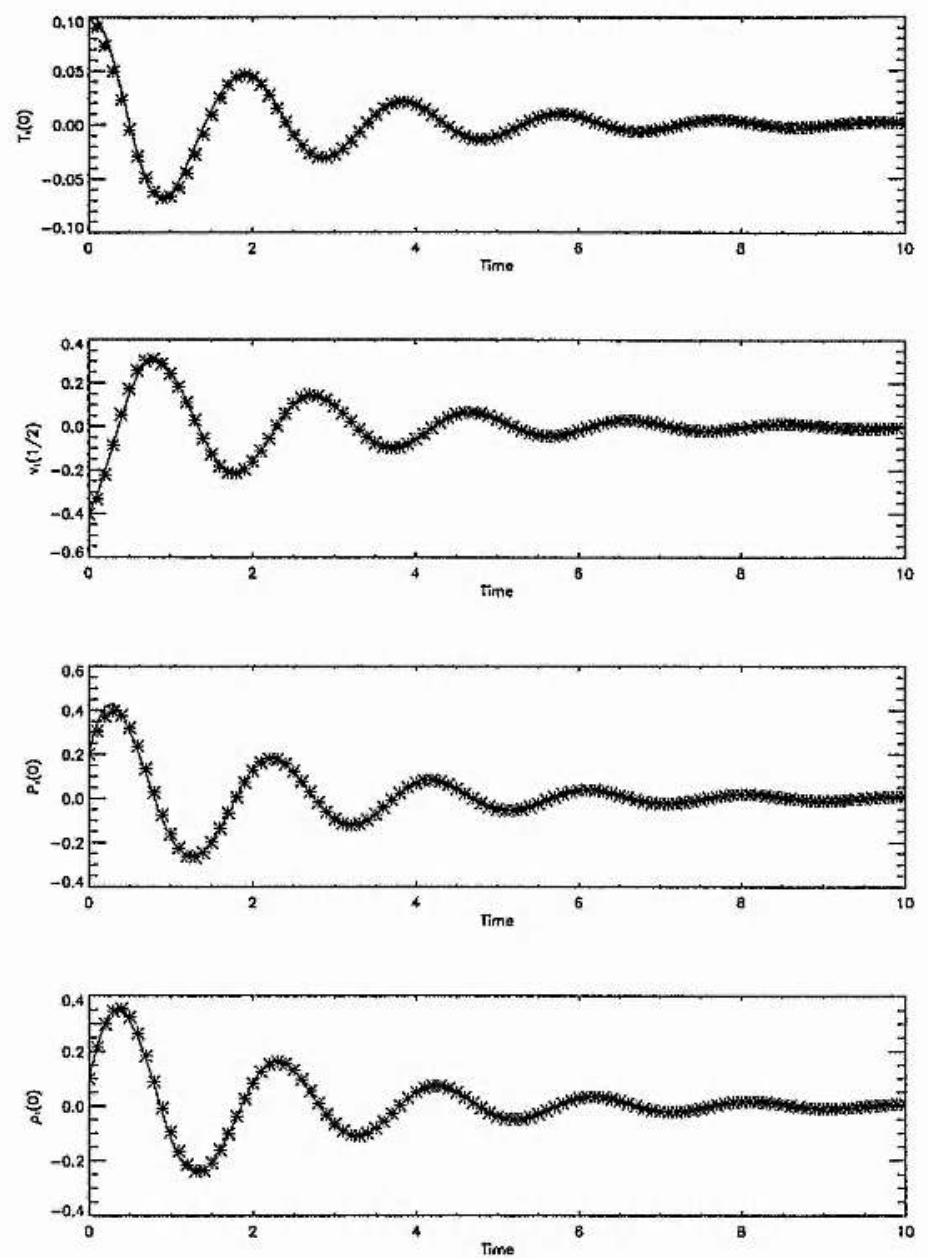

Figure 6.10: Analytical (***) and numerical calculations $(-)$ for the relaxation of the temperature $\left(T_{1}\right)$, velocity $\left(v_{1}\right)$, pressure $\left(p_{1}\right)$ and density $\left(\rho_{1}\right)$ perturbations for $\epsilon_{0}=1, b=1, \eta=0.01$ and $A=0.1$.

$$
\begin{gathered}
p_{1}(x, t)=\frac{A \mathrm{e}^{\sigma_{r} t}}{(\gamma-1) \pi^{2}}\left[\left\{\left(\sigma_{r}+\pi^{2} \eta\right)\left(\left(b-\pi^{2}\right) \epsilon_{0}-\sigma_{r}\right)+\sigma_{i}^{2}\right\} \cos \left(\sigma_{i} t\right)\right. \\
\left.-\left\{\left(b-\pi^{2}\right) \epsilon_{0}-2 \sigma_{r}-\pi^{2} \eta\right\} \sigma_{i} \sin \left(\sigma_{i} t\right)\right] \cos (\pi x), \\
\rho_{1}=p_{1}-T_{1} .
\end{gathered}
$$

$\sigma_{r}$ and $\sigma_{i}$ are calculated from the analytical solution to (6.38) and the initial condition for the numerical code is obtained by setting $t=0$ in the above equations. Figure 6.10 shows the evolution of $T(0), p(0), \rho(0)$ and $v(1 / 2)$ for $b=1, \epsilon_{0}=1$ and $\eta=0.01$. Again, there is excellent agreement between the two methods. 


\subsubsection{A Non-isothermal Equilibrium}

Consider lowering the footpoint temperature such that $T_{e}=0.8$, with $p_{e}=1$ and $\rho_{e}=p_{e} / T_{e}$. The simplified energy equation (6.27) allows for an analytical solution for the thermal equilibria across the system. Thus, for the static solution we have,

$$
\frac{\partial^{2} T}{\partial x^{2}}=b(1-T)
$$

which can be solved to give

$$
T(x)=1+\frac{\left(T_{e}-1\right)}{\cos (\sqrt{b} / 2)} \cos (\sqrt{b} x) .
$$

The numerical code is given the initial set of conditions

$$
\begin{aligned}
T(x, 0) & =T_{\text {sum }}+4\left(T_{e}-T_{\text {sum }}\right) x^{2}, \\
p(x, 0) & =1 \\
v(x, 0) & =0 \\
\rho(x, 0) & =\frac{p(x, 0)}{T(x, 0)},
\end{aligned}
$$

where $T_{\text {sum }}=0.75, b=1, \epsilon_{0}=1, \gamma=5 / 3$ and $\eta=0.1$ and allowed to relax. Figure 6.11 shows the "loop" summit temperature, pressure and density and the velocity at the "loop" footpoint initially overshooting but eventually settling down to steady values. The excellent agreement between the numerically calculated steady state and the analytical result (6.57) is shown in Figure 6.12.

\subsection{Time-Dependent Heating of an Initially Static Coronal Loop}

A more realistic form of the radiation-heating term in the energy equation is

$$
p^{2} \chi T^{\alpha-2}-H
$$

and with a $T^{5 / 2}$ dependence upon conduction, we return to the full set of coupled non-linear pde's (6.19)-(6.22).

Consider a coronal loop with $b=0.5(\sim 60 \mathrm{Mm})$ with $T_{\mathrm{e}}=0.01\left(=10^{4} \mathrm{~K}\right), p_{e}=1$ $(=0.01 \mathrm{~Pa})$ and $\rho_{e}=p_{e} / T_{e}$. For this set of parameters, $0.05 \leq \epsilon_{0} \leq 0.1$ and we shall set $\eta=0.1$. We will continue to use the Two Range Radiative Loss Function (3.3) as an approximation to the optically thin radiation in the corona with $T_{r}=0.011$ (in units of $10^{6} \mathrm{~K}$ ) as before. We shall use the time-dependent heating term

$$
H=H_{0}(1+\sin (\omega t)),
$$



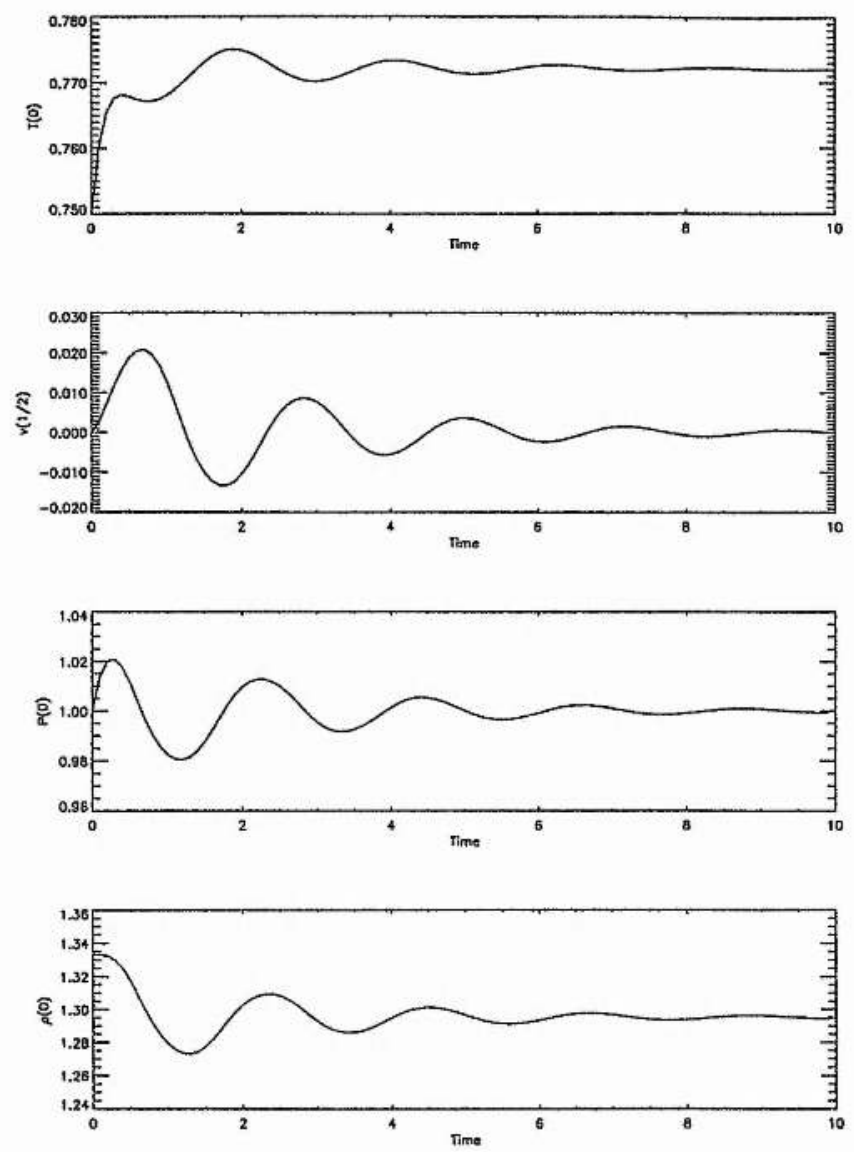

Figure 6.11: Numerical calculation for the relaxation of the temperature $(T(0))$, velocity $(v(1 / 2))$, pressure $(p(0))$ and density $(\rho(0))$ for $\epsilon_{0}=1, b=1$ and $\eta=0.1$.

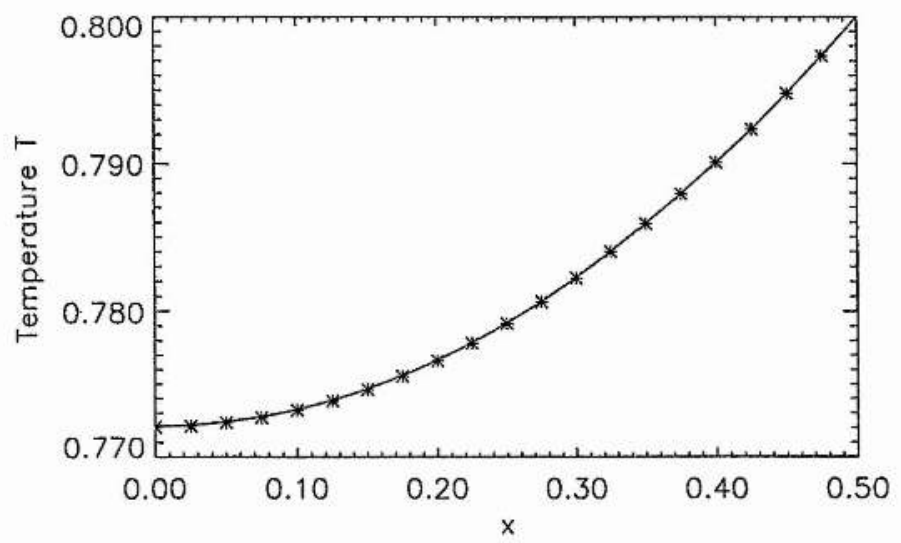

Figure 6.12: Comparison of the numerically $(-)$ and analytically $\left({ }^{* * *}\right)$ calculated thermal equilibria profiles. 
where $H_{0}=50\left(\sim 2 \times 10^{25}\right.$ ergs $\left.\mathrm{s}^{-1}\right)$ is the average heat deposited in the loop per heating cycle and $\omega$ is a frequency to be varied. The reason for using this heating function is set out in Section 4.3. Thus we can have a direct comparison with the results obtained for the isobaric case (see Chapter 4) with those produced with the inertial terms included.

In order to test the validity of the isobaric assumption upon the time-dependent heating scenario, it is important to note that in the isobaric case, time $t$ was nondimensionalised against the conductive timescale $\left(\tau_{\text {cond }}\right)$ while in the inertial case the sound travel time $\left(\tau_{s}\right)$ is used (see Section 6.1). Now since we have

$$
\epsilon_{0}=\frac{\tau_{s}}{\tau_{\text {cond }}}
$$

then for the heating frequency in the isobaric case,

$$
\omega_{i s o}=\omega \tau_{c o n d},
$$

while for the case with the inertial terms included we have

$$
\omega_{\text {inert }}=\omega \tau_{s},
$$

where $\omega$ is the dimensionalised frequency. We must have

$$
\omega_{\text {inert }}=\epsilon_{0} \omega_{i s o} .
$$

to compare the frequencies. Note that when $\omega=0$, the steady state solution to (6.19) - (6.22) has the same equilibria as in Section 3.2 and therefore all the results from Chapter 3 are applicable here. For a convincing confirmation of the validity of the isobaric assumption to coronal loops, a heating frequency must be found such that the pressure variation is small compared to the corresponding temperature and density changes. Figure 6.13 shows the evolution of the temperature, pressure, velocity and density along half the loop length for three different times $\left(t_{1}<t_{2}<\right.$ $\left.t_{3}\right)$ as the heating function increases from its minimum value $\left(H\left(t_{1}\right) \sim 0\right)$ to its maximum value $\left(H\left(t_{3}\right) \sim 100\right)$ for $\epsilon_{0}=0.1$ and $\omega_{\text {inert }}=2.5\left(\omega_{\text {iso }}=25\right)$.

The temperature along the loop rises as the heating function increases. $p(x)$ is practically constant along the loop length at a particular time except where it is tied at $p(1 / 2)=1$. The velocity changes direction as the heat function increases and its value is small at the loop footpoint (though not zero) - this is a consequence of the fact that hot, light material is flowing into the cool, dense material of the chromosphere. The density along the loop changes by only a small amount. Figure 6.14 shows the evolution of the summit temperature $T(0)(-)$, pressure $p(0)(-$ - -) and density $\rho(0)(. .--)$ for the above case. The variation in the pressure is 


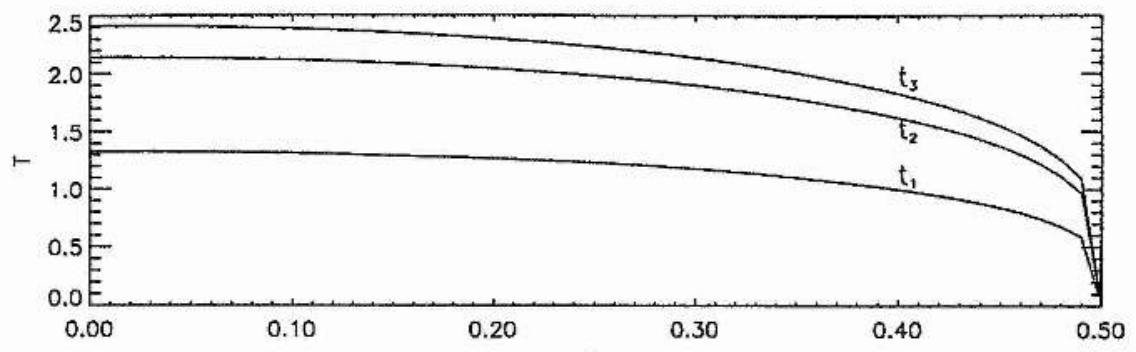

(a)

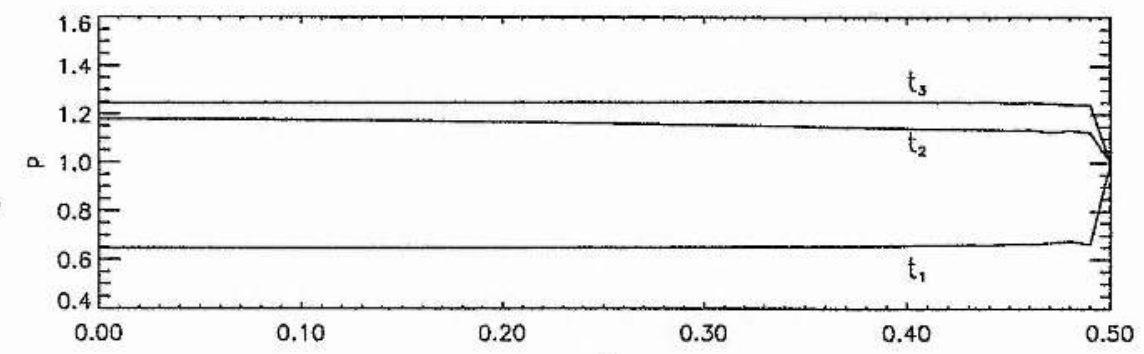

(b)

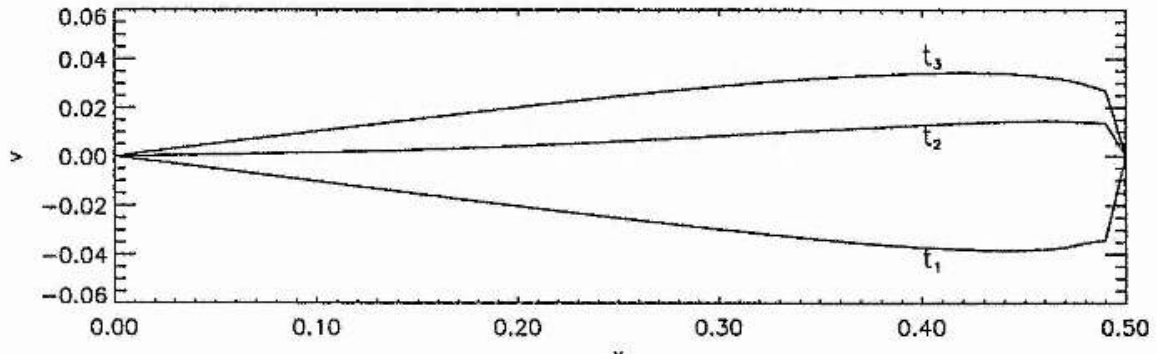

(c)

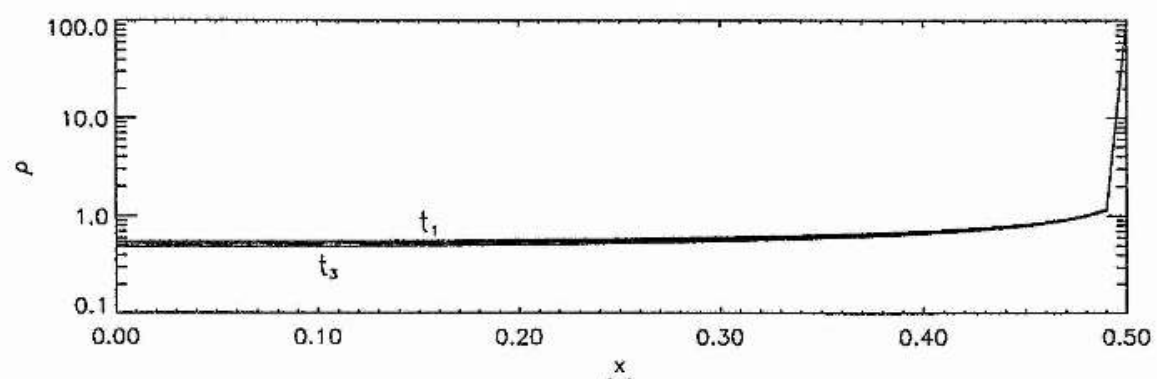

(d)

Figure 6.13: The evolution of the (a) temperature, (b) pressure, (c) velocity and (d) density along half the loop length for three different times $\left(t_{1}<t_{2}<t_{3}\right)$ as the heating function increases from its minimum to its maximum value for $\epsilon_{0}=0.1$ and $\omega_{\text {inert }}=2.5$. 


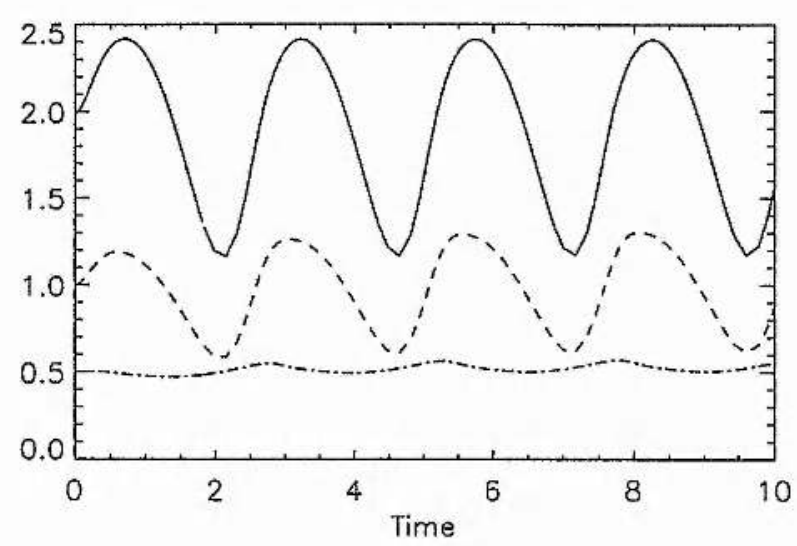

Figure 6,14: The evolution of the summit temperature $T(0)(-)$, pressure $p(0)(--)$ and density $\rho(0)(-,--)$ for $\epsilon_{0}=0.1$ and $\omega_{\text {inert }}=2.5$.

much greater than for the density and therefore the system appears to be evolving isochorically rather than isobarically. If we compare the behaviour of $T(0)$ with its isobaric counterpart $\left(\omega_{i s o}=25\right)$ (see Figure 6.15), the summit temperature profiles match-up very well. Thus, the temperature follows very closely the changes in the heating function and seems to not be particularly influenced by the corresponding variations in the pressure and density.

There are two possible ways of trying to achieve a closer comparison with the isobaric case (i) by decreasing $\epsilon_{0}$ or (ii) by reducing the heating frequency $\omega_{\text {inert }}$.

\subsubsection{Decreasing $\epsilon_{0}$}

If $\epsilon_{0}$ is reduced then this is effectively reducing the acoustic timescale. Figure 6.16 shows the fractional change in the various plasma properties away from their equilibrium values for $\epsilon_{0}=0.001$ with $\omega_{\text {inert }}=0.025\left(\omega_{\text {iso }}=25\right)$. The change in the pressure is much smaller than the corresponding temperature and density variations and the system does indeed reduce to isobaric behaviour when the sound travel timescale is short enough.

\subsubsection{Reducing $\omega_{\text {inert }}$}

When reducing the heating frequency, there is a "trade-off" between approaching an isobaric situation and remaining above some critical frequency value, below which the loop plasma cools to chromospheric values. Figure 6.17 presents the evolution of $T(0), \rho(0)$ and $p(0)$ with respect to time for $\epsilon_{0}=0.1$ and $\omega_{\text {inert }}=0.4\left(\omega_{\text {iso }}=4\right)$; the 


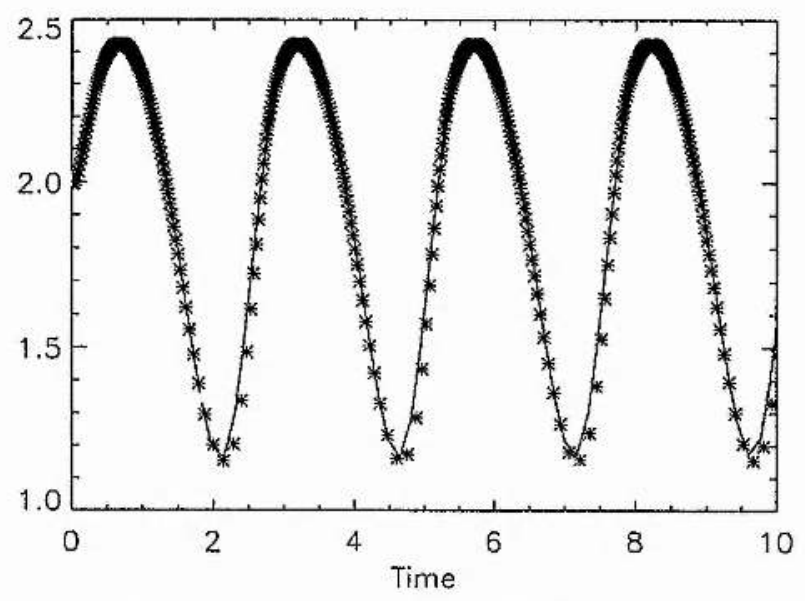

Figure 6.15: Comparison of the summit temperature $T(0)(-)$ with the previous isobaric result $(* * *)$ for $\epsilon_{0}=0.1$ and $\omega_{\text {inert }}=2.5\left(\omega_{i s o}=25\right)$.

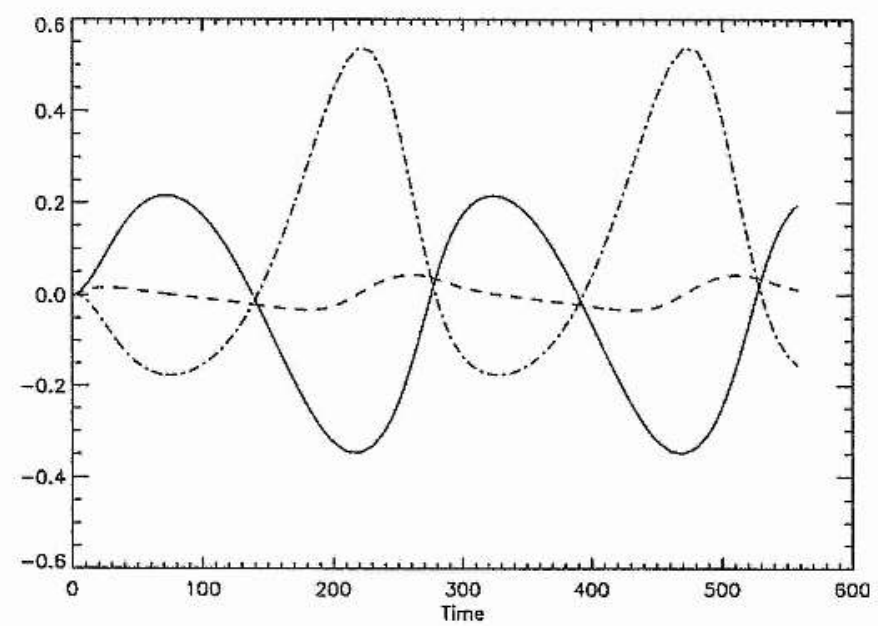

Figure 6.16: Fractional change from the equilibrium values for the summit temperature $T(0)(-)$, pressure $p(0)(--)$ and density $\rho(0)(-.--)$ with $\epsilon_{0}=0.001$ and $\omega_{\text {inert }}=0.025\left(\omega_{i s o}=25\right)$. 


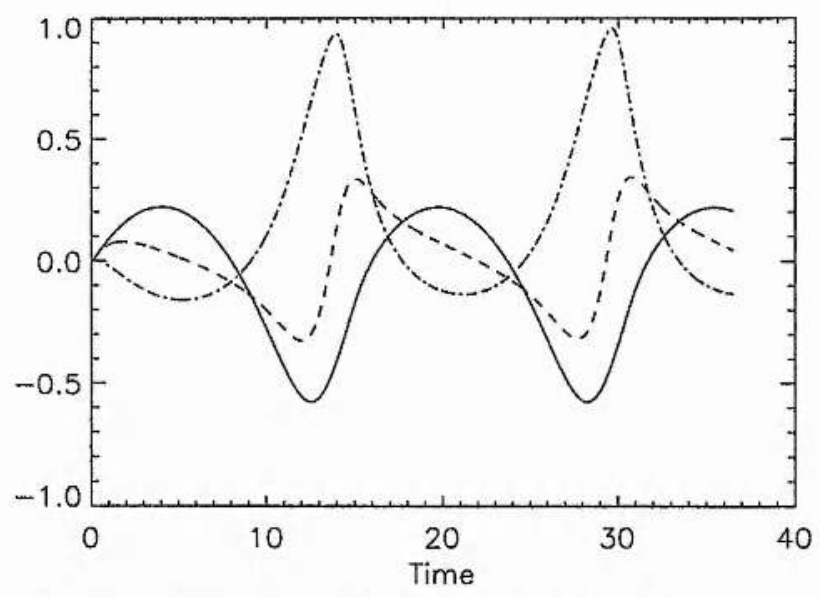

Figure 6.17: Fractional change from the equilibrium values for the summit temperature $T(0)(-)$, pressure $p(0)(--)$ and density $\rho(0)(-.--)$ for $\epsilon_{0}=0.1$ and $\omega_{\text {inert }}=0.4\left(\omega_{\text {iso }}=4\right)$.

fractional change in the pressure has decreased from Figure 6.14 but is still sizeable. If $\omega_{\text {inert }}$ is decreased further, a cool region of dense plasma travels in from the loop footpoint. Unfortunately, the numerical code does not appear to be robust enough to deal with this high density region and it breaks down when the cool region forms. The reason for this problem seems to be the dramatic change in the radiation as the temperature passes through $T=T_{r}$ and falls into the lower radiative loss region. Other numerical runs have shown that if $T_{r}$ and $T_{e}$ are increased (to $T_{r} \approx 0.1$ and $T_{e} \approx 0.05$ say), the numerical algorithm can cope with the change in the radiative loss - if the heating frequency is too low, the loop cools to approximately $T_{e}$ along its entire length. Note that in this case, the maximum value of the radiative loss is nearly two orders to magnitude less than when $T_{r}=0.01$. Thus, the same physical process should be taking place with the actual physical parameters. The refinement of the computational code is work to be undertaken in the future.

\subsection{Conclusions}

The usefulness of the isobaric assumption to coronal loop structures was investigated by including the inertial terms in the equation of motion. A simplified form of the energy equation was introduced which allowed for the analytical investigation of the temperature, density, pressure and velocity of the plasma through a derived dispersion relation. This relation was investigated for different parameter values and the predicted asymptotic behaviour displayed all the observed features generated numerically. Indeed, Figure 6.10 demonstrates the excellent agreement between the 
two approaches.

When applied to a typical coronal loop, it was found that although there is a variation in pressure throughout the periodic process, the isobaric assumption appears to be a reasonable simplification which provides accurate estimates of the temperature profile. It was also found that the evolution of the physical parameters along the loop reduced to an isobaric situation provided the acoustic timescale was small enough. 


\section{Chapter 7}

\section{Summary and Further Work}

A little learning is a dang'rous thing,, Drink deep or taste not ..... (Alexander Pope)

\subsection{Summary}

To astronomers, the Sun is a fairly ordinary star, a massive ball of gas held together and compressed under its own gravitational attraction. However, the proximity of the Earth to the Sun means that it is a fertile laboratory that poses many interesting problems for the theorist. Better and better observations from ground and space based instruments are helping to confirm (and refute) theoretical models of structures on the Sun and there are many features which still prove very difficult to understand.

One of the most perplexing of Solar Physics problems has been to explain the high temperature of the corona $\left(\sim 2 \times 10^{6} \mathrm{~K}\right)$ as compared to the solar surface $\left(10^{3}-10^{4} \mathrm{~K}\right)$. Many methods have been suggested to supply the heat to this tenuous medium to balance the losses due to radiation, conduction and plasma mass flows (see Section 4.2). However, it is likely that none of these models will provide a constant supply of energy to the corona - the energy will be intermittant or time-dependent. This has been the main topic of investigation in this thesis.

Observations of the corona in soft X-rays has revealed a complex network of magnetic loop structures in the solar atmosphere. Using the governing fluid equations along a coronal loop (Chapter 2), the thermal equilibria profiles were derived under two simplifying assumptions; (i) the system is isobaric and (ii) a Two Range Radiative Loss Function was introduced as an approximation to the optically thin radiation (Chapter 3). 
A heating function that evolved sinusoidally in time was investigated in Chapter 4 and it was found that the heat must be supplied frequently enough to maintain the loop at coronal temperatures; that is, there exists a critical frequency which must be exceeded to maintain the loop at $\approx 10^{6} \mathrm{~K}$, otherwise the loop cools along its length to chromospheric temperatures. If the loop does cool, coronal temperatures can only be reached once again when the average heating is increased dramatically; keeping the average amount of heat deposited in the loop the same but increasing the frequency simply means that the temperature along the loop length oscillates about the lower isothermal states. As expected, the same results occur when the Hildner (1974) estimates for the radiative losses are introduced. It was found in this case that the critical frequency was approximately the order of (conductive timescale) ${ }^{-1}$. Even the recent radiative loss curve of Cook et al, (1989) will show the same increase with temperature when evaluated at constant pressure. Thus, although the radiative losses are smaller, a critical frequency would still exist. The form of the differential emission measure was also calculated - an increase in the DEM below $10^{5} \mathrm{~K}$ was found for a heating frequency just above the critical value.

Other forms of time-dependent heating investigated (Chapter 5) included pulse heating as well as random releases of energy quanta of various sizes using a Poisson Distribution Function. It was found that for this particular statistical process, only the rapid release of nanoflare-sized energy bursts every $\sim 10$ s could keep the coronal loop at its hot solution.

The usefulness of the isobaric assumption to the corona was assessed in Chapter 6 by including the inertial terms in the equation of motion and therefore making it necessary to track the sound waves travelling back and forth across the system. It was found that the temperature evolution of the response of the plasma to a timevarying heating supply can be approximated to a very high degree by the simple isobaric case, even though the overall behaviour of the system appears to be closer to isochoric than isobaric. In this way we see that the temperature changes are mainly governed by the variations in the heating term. However, isobaric case is reproduced when the acoustic timescale is short enough.

It must be remembered that in order to understand such a complicated system (as the response of a plasma contained within a solar coronal magnetic loop to a time-dependent energy supply), it is necessary to produce some basic models of the phenomenon which include what are considered to be all the important features this is what we have investigated above. However, a large number of modifications can be made to these models and several of these are discussed below. They cover the areas of coronal loop models, time-dependent heating, improving the numerical 
algorithm and implications on SOHO observations.

\subsection{Addition of Gravity}

Including gravity into the equation of motion gives

$$
\frac{\partial(\rho v)}{\partial t}-\frac{\partial\left(\rho v^{2}\right)}{\partial x}=-\frac{\partial p}{\partial x}+\rho g(x)+\nu \frac{\partial^{2} v}{\partial x^{2}}
$$

with

$$
g(x)=g_{0} \sin \pi x
$$

for a semi-circular curvature of the magnetic loop structure and where $g_{0}=-274$ $\mathrm{ms}^{-2}$ is the local gravitational acceleration at the surface of the Sun. The pressure along the loop now falls from the footpoint to the summit.

The effect of gravity on the time-dependent heating scenario can be roughly estimated as follows. For loop temperatures of $\sim 2 \times 10^{6} \mathrm{~K}$, the pressure scale height is $\sim 10^{5} \mathrm{~km}$ but this reduces to $\sim 10^{4} \mathrm{~km}$ at a temperature of $10^{5} \mathrm{~K}$. Thus if the heating occurs sufficiently frequently that the average loop temperature along the loop is greater than $10^{6} \mathrm{~K}$, then neglecting gravity is a valid assumption. However, a lower frequency, which in the isobaric case would produce a cool temperature region of dense plasma in the loop, will have the "condensed" material rapidly draining back onto the chromosphere, if gravity were included. There would be a reduction in the loop density and therefore a reduction in the magnitude of the radiation term. The loop will take longer to cool and what should be observed is a drop in the critical frequency.

\subsection{Addition of Chromosphere}

The addition of a realistic chromospheric response is very important. Mariska et al (1982) argue that the chromosphere should behave like a loaded spring with the overlying layers providing most of the loading. Rapid changes in the pressure caused by time-dependent heating would cause the chromosphere to rise and fall in response, the upper boundary of the chromosphere being defined as the height at which the temperature is $\sim 10^{4} \mathrm{~K}$. However, these authors impose a chromosphere that is isothermal which requires the plasma radiation and heating effectively to balance. This is likely to be an oversimplification. One possible way to model the chromosphere would be to implement Newton's Law of Cooling below $10^{4} \mathrm{~K}$ or to try properly to model the chromospheric radiation losses (as in Peres et al, 1982), remembering that in this region, optically thick effects are important. 
This important modification to the model developed in this thesis would require the extension of the non-linear algorithm (see Appendix $G$ ) to deal properly with the rapid changes from the corona, through the transition region and into the newly defined chromosphere. In the work done so far, the uniform spatial grid used has given unnecessarily high resolution in the corona in order to provide a fine enough spacing in the transition region - this has required prohibitive amounts of computational time. Thus, an adaptive eulerian grid would need to be implemented.

\subsection{Variation in Loop Cross-Sectional Area}

Figure 2.3 presented a sketch of a magnetic flux tube having risen up through the dense photosphere and chromosphere and spread out into the less dense corona. Increases in the loop cross-sectional area $(A(s))$ could be introduced at a single point (Hood and Anzer, 1988) or by an exponential expansion along the loop as

$$
A(s)=\frac{A_{0}-A_{b}}{2}\left[\tanh \left(\frac{4\left(s-s_{0}\right)}{l_{0}}\right)+1\right]+A_{b},
$$

where $A_{0}$ and $A_{b}$ are the summit and base cross-sectional areas respectively, $s$ is the distance along the loop from the footpoint to the apex, $s_{0}$ is the distance above the footpoint where the expansion occurs while $l_{0}$ is the distance over which the loop expands (Brown, 1995). Figure 7.1 shows the variation in the area (normalised against the base area $A_{b}$ ) along a $30 \mathrm{Mm}$ loop for various values of $s_{0}$ and $l_{0}$. In (i), $s_{0}=2 \mathrm{Mm}$ and $l_{0}=2 \mathrm{Mm}$ such that the main loop expansion occurs in the low corona whereas in (iii), $s_{0}=20 \mathrm{Mm}$ and $l_{0}=15 \mathrm{Mm}$, giving an expansion much higher up in the coronal atmosphere.

In all cases of a change in loop area, there will be a corresponding change in the magnetic field structure such that the thermal equilibria along the loop would now be

$$
\frac{1}{A} \frac{d}{d x}\left(\kappa_{0} T^{5 / 2} A \frac{d T}{d x}\right)=B \frac{d}{d x}\left(\frac{\kappa_{0} T^{5 / 2}}{B} \frac{d T}{d x}\right)=\left[\rho^{2} \chi T^{\alpha}-H\right],
$$

where $B$ is the magnetic field strength (Steele and Priest, 1991).

\subsection{Random Heating Events}

The Poisson process outlined in Section 5.4 is a simple, first approximation to modelling a heat source that has a random amount of energy deposited in a fixed time interval. Other methods could include:- 


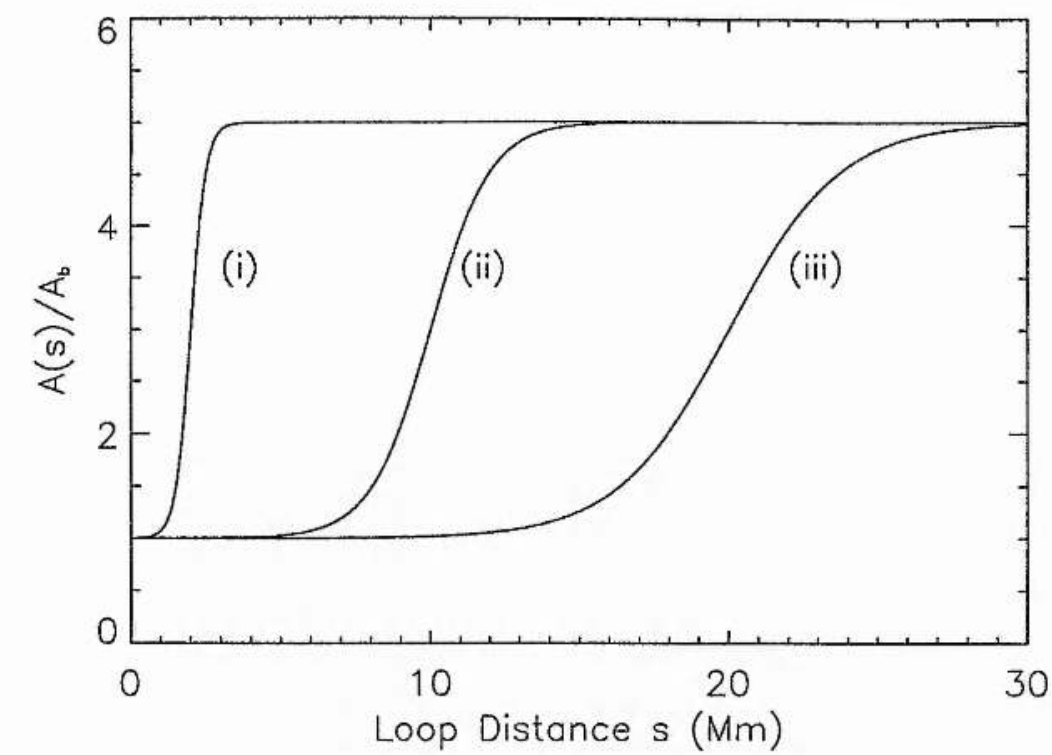

Figure 7.1: Variation in loop cross-sectional area with distance from the loop footpoint for (i) $s_{0}=2 \mathrm{Mm}, l_{0}=2 \mathrm{Mm}$; (ii) $s_{0}=10 \mathrm{Mm}, l_{0}=10 \mathrm{Mm}$ and (iii) $s_{0}=20 \mathrm{Mm}, l_{0}=15 \mathrm{Mm}$.

- random amounts of energy quanta released at random time intervals - this introduces the possibility of overlapping energy release events.

- the importance of the previous history of the heating bursts. For example, if there is a large energy event at one time, the system may need time to recover and build up its "energy store" once again before releasing the next heat pulse. This could happen if the magnetic field was required to be sheared or twisted by a certain amount before the energy is released.

- the energy need not necessarily be quantized (into nanoflare-sized chunks for example). Thus, we could have

1. a rectangular probability density function of the form

$$
\text { p.d.f. }=\frac{1}{2 \mathrm{H}_{0}} \text { on }\left[0,2 \mathrm{H}_{0}\right]
$$

where $H_{0}$ is the average amount of heat deposited in the corona. The energy release during each event $H$ would then be

$$
H=2 H_{0} U
$$

where $U$ is a random number.

2. a Normal p.d.f. about $H_{0}$ with standard deviation $\sigma$. This particular distribution leads to the possibility of a negative energy input depending on the size of $\sigma$ - this could simply be truncated to give zero. 


\subsection{Spatially-Dependent Heating}

Asymmetric heating of coronal loops has been considered by other authors to try and model the observed flows in the corona (Mariska, 1987 and references therein). The heating is usually taken to be constant with a certain weighting down one leg of the loop, with no mention made as to how this asymmetric energy deposition is occurring. However, as argued in Section 4.2.5, a time-dependent heating source is more realistic.

Thus, for example, wave heating of a loop might intermittantly dump more energy at the loop footpoints while a magnetic reconnective event could release its energy preferentially at the loop summit. Nanoflares, on the other hand, may deposit their energy on some small lengthscale anywhere along the loop.

\subsection{Two- and Three-Dimensional Modelling}

A realistic prediction of the $\mathrm{X}$-ray emission of coronal loops requires two- and threedimensional versions of the numerical model in order to allow the integrated line of sight values to be calculated. This would be undertaken in several steps. First of all, model two- and three-dimensional potential and force-free equilibria would be set up, both for the quiet Sun and also for active regions. Then realistic potential and forcefree models of observed regions would be developed. In each case, it will be necessary to solve the thermal equations, along each field line, for the plasma response in such equilibria to different kinds of heating events and the results compared with observations. In a final step, the same procedure would be undertaken with a fully MHD three-dimensional code with complete energetics.

\subsection{Implications for SOHO Observations}

When the SOHO satellite begins sending data back to Earth, the results produced will have an enormous impact on the theoretical solar community. As indicated in Chapter 1, understanding coronal heating is one of the principle objectives of this space-based mission, particularly using the SUMER and CDS instruments. In Chapter 4, possible observational evidence for the different heating theories was thoroughly discussed and therefore we concentrate here upon proposed studies relevant to the research in this thesis as well as suggest possible future observing sequences for the above instruments. 


\subsubsection{SUMER and CDS Studies}

The SUMER and CDS experiments are highly complementary in terms of the scientific objectives they can achieve; CDS obtains data on hotter lines while SUMER observes somewhat cooler lines. Thus many proposed observing projects plan a joint SUMER-CDS programme to look at selected regions of the Sun.

There is a concentration of studies to detect small $\left(\sim 10^{24}-10^{25} \mathrm{ergs}\right)$ flare-like events. With its ability to study plasma flows, temperatures and densities in the upper chromosphere and lower corona, SUMER has been suggested as a possible instrument to detect nanoflares by searching for small flare events in active regions and then investigating the various $\mathrm{Fe}$ line intensities to determine the mass motions present (Mason, 1995; Doschek, 1995).

Hansteen (1995) proposes to look for evidence of downward propagating progression waves in the corona that would be the result of a sudden deposition of energy while Klimchuk and Harrison (1995) suggest that if CDS is pointed at a well-defined coronal loop, the filling factor could be calculated by comparing density measurements from line ratio and emission measure calculations (see Cargill 1993).

Given the limited temporal resolution and temperature range of previous observing instruments, Harrison and Priest (1995) and Harrison and Gurman (1995) believe that CDS could detect and track elementary heating events in the solar atmosphere. Poletto et al (1995) on the other hand, suggest that it is more than likely that only the largest events in this particular family of flares will be individually recognised. Following on from Kopp and Poletto (1993), they will use CDS to measure the brightness over an area which may include a few magnetic loops but with a spatial resolution insufficient to resolve an individual loop. Using this data, they will attempt to calculate how the nanoflare energies are distributed and compare the results with their theoretical model.

It will be interesting to see if the "elusive" nanoflare can finally be discovered and whether there exists a lower energy threshold (do picoflares exist and contribute to coronal heating?).

An emission measure study of the quiet Sun has been put forward by $M^{c}$ Whirter (1995) to derive physical parameters for the solar atmosphere over the temperature range of $3 \times 10^{4}-3 \times 10^{6} \mathrm{~K}$ so that meaningful comparisons can be made with theoretical models based on energy and pressure balance. This could also give some clues as to the nature of the heating mechanism.

Wave theories of coronal heating will be investigated by $\mathrm{M}^{\mathrm{c}}$ Clements et al (1995) and Doyle (1995). The former will try to detect low frequency waves in the corona by using CDS to identify a large coronal loop at the centre of the solar disc - SUMER 
will then be pointed at the loop footpoint and the magnetic field measured as the loop tracks across the disc to the limb. The latter will look for subsurface turbulent motions (chromospheric oscillations) as a source of coronal heat energy. Both these studies could show the importance of preferentially depositing the heat energy in the loop footpoints.

\subsubsection{Possible Observations}

These could include;

- the time-dependent variation in the temperature along a coronal loop. This could be compared with the numerical model results such as in Figure 4.15 to.deduce the possible frequency of heating mechanisms. This could also give some observational evidence for the time-varying heat function suggested by Wright and Rickard (1995).

- if the nanoflare is "found", what is its rate of occurrence? Does it occur frequently enough to provide the energy necessary to maintain a typical coronal loop at typical coronal temperatures? At what height in the atmosphere do they exist?

- the time-dependent nature of the differential emission measure. The results shown in Section 4.7 follow the evolution of the DEM for a periodic heating function, the size of the variations depending on the frequency of the heating process. This could be compared with CDS and SUMER observations.

Throughout any further work, it will be very important to relate and compare theory with actual observations. The SOHO Mission is a timely opportunity for both theorists and observers to work together to improve our knowledge and understanding of many aspects of the Sun. 


\section{Appendix A}

\section{Critical Point Analysis of an Autonomous System}

In this Appendix we will investigate the nature of the critical points in the $(y, \Phi)$ space of the autonomous set of equations,

$$
\begin{aligned}
\frac{d y}{d x} & =\Phi, \\
\frac{d \Phi}{d x} & =\frac{7 b}{2}\left[\chi y^{2(\alpha-2) / 7}-H_{0}\right],
\end{aligned}
$$

which for $H_{0}<y_{r}{ }^{-1 / 2}$ are

$$
\left(y_{h}, 0\right)=\left(H_{0}^{-2}, 0\right) \text { and }\left(y_{c}, 0\right)=\left(H_{0} y_{r}{ }^{3 / 2}, 0\right) \text { respectively } .
$$

To investigate the local behaviour of the solution near $y=y_{h}$, let $\left(y_{h}, 0\right) \approx\left(H_{0}{ }^{-2}+\right.$ $\left.\epsilon_{1}, \epsilon_{2}\right)$ where $\epsilon_{1}$ and $\epsilon_{2}$ are small quantities. Then approximate the differential equations (A.1) and (A.2) by

$$
\begin{aligned}
\dot{\epsilon_{1}} & \sim \epsilon_{2}, \\
\dot{\epsilon_{2}} & \sim \frac{7 b}{2}\left[\left(H_{0}{ }^{-2}+\epsilon_{1}\right)^{-1 / 2}-H_{0}\right],
\end{aligned}
$$

with $\chi=1$ and $\alpha=1 / 4$ for $y_{h}>y_{r}$. A Taylor series expansion on the right-hand side of (A.4) gives

$$
\dot{\epsilon_{2}} \sim-\frac{7 b H_{0}{ }^{3}}{4} \epsilon_{1} .
$$

Dividing (A.5) by (A.3) gives

$$
\frac{d \epsilon_{2}}{d \epsilon_{1}} \sim-\frac{7 b H_{0}^{3}}{4} \frac{\epsilon_{1}}{\epsilon_{2}}
$$


which can be integrated to give

$$
\epsilon_{2}^{2}+\frac{7 b H_{0}^{3}}{4} \epsilon_{1}^{2}=C
$$

where $C$ is a constant of integration. This is the equation of an ellipse and therefore this critical point is a centre point. The direction of rotation of the closed trajectories around the centre is obtained by setting $\epsilon_{2}=0$ and $\epsilon_{1}>0$ and seeing if $\dot{\epsilon}_{2}$ is positive or negative. In this case $\dot{\epsilon}_{2}$ is negative and therefore the rotation is clockwise.

Similarly to investigate the local behaviour near the second critical point at $y=$ $y_{c}$, let $\left(y_{c}, 0\right) \approx\left(H_{0} y_{r}^{3 / 2}+\epsilon_{1}, \epsilon_{2}\right)$ and approximate the differential equations (A.1) and (A.2) by

$$
\begin{aligned}
\dot{\epsilon_{1}} & \sim \epsilon_{2}, \\
\dot{\epsilon_{2}} & \sim \frac{7 b}{2}\left[\left(H_{0} y_{r}{ }^{3 / 2}+\epsilon_{1}\right) y_{r}{ }^{-3 / 2}-H_{0}\right],
\end{aligned}
$$

where $\chi=y_{r}{ }^{-3 / 2}$ and $\alpha=11 / 2$ for $y_{c}<y_{r}$. Expanding the right-hand side of (A.9) gives

$$
\dot{\epsilon_{2}} \sim \frac{7 b}{2 y_{r}{ }^{3 / 2}} \epsilon_{1}
$$

As before, equations (A.8) and (A.10) can be integrated to give

$$
\epsilon_{2}{ }^{2}-\frac{7 b}{2 y_{r}{ }^{3 / 2}} \epsilon_{1}{ }^{2}=C
$$

which is the equation of an hyperbola. Thus the critical point is a saddle point. 


\section{Appendix B}

\section{Analytical Calculation for the Dependence of The Summit Temperature upon Loop Length}

The advantage of using the Two Range Radiative Loss Function (3.3) is that an analytical solution to the thermal equilibria (3.2) can be found. Figure B.1 shows the two possible thermal equilibrium loop structures which depends on the summit temperature $T_{0}$ (which in turn depends on the loop length (through $b$ ) and the average heating value $H_{0}$.

(i) $T_{0}>T_{r}>T_{e}$;

This loop has two different temperature regions corresponding to the different values of $\chi$ and $\alpha$ for the radiative function on either side of $T=T_{r}$. For $T>T_{r}$,

$$
\frac{d^{2} y}{d x^{2}}=y^{\prime \prime}=\frac{7 b}{2}\left[y^{-1 / 2}-H_{0}\right]
$$

from equation (3.2). Multiplying each side by $d y / d x=y^{\prime}$ and integrating gives

$$
\left(y^{\prime}\right)^{2}=7 b\left(2 y^{1 / 2}-H_{0} y+C\right)
$$

where $C$ is a constant of integration. We know from the boundary condition (3.5) that $y^{\prime}=0$ when $y=y(0)=y_{0}$ for this upper region and thus,

$$
\left(y^{\prime}\right)^{2}=7 b\left(2 y^{1 / 2}-H_{0} y-2 y_{0}{ }^{1 / 2}+H_{0} y_{0}\right)
$$

which can be written as

$$
y^{\prime}=-\sqrt{\frac{7 b}{H_{0}}}\left[\left(1-H_{0} y_{0}{ }^{1 / 2}\right)^{2}-\left(1-H_{0} y^{1 / 2}\right)^{2}\right]^{1 / 2},
$$




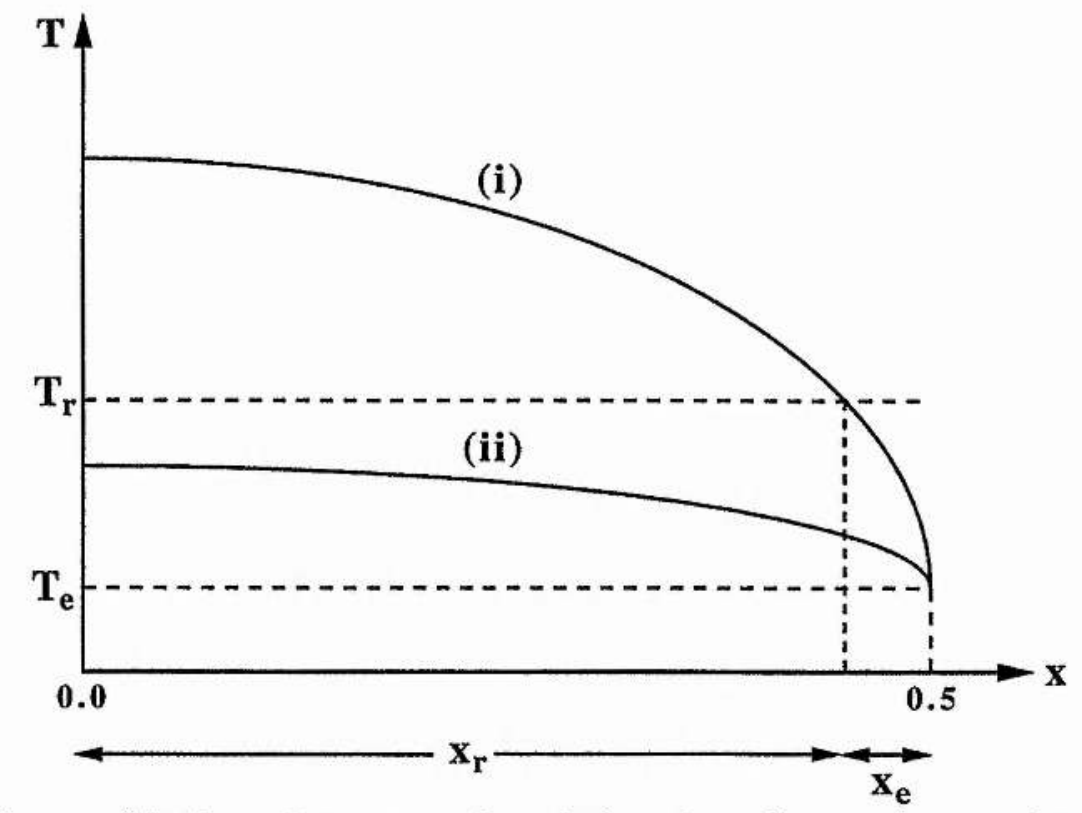

Figure B.1: Two possible thermal structures along the loop depending on the summit temperature $T(0)=T_{0}$; (i) $T_{0}>T_{r}>T_{e}$ and (ii) $T_{0}, T_{e}<T_{r}$.

the negative root chosen as the temperature gradient is always negative. We must have

$$
\left(1-H_{0} y_{0}^{1 / 2}\right)^{2}-\left(1-H_{0} y^{1 / 2}\right)^{2}>0
$$

but if

$$
\left(1-H_{0} y_{0}^{1 / 2}\right)>\left(1-H_{0} y^{1 / 2}\right)
$$

then

$$
y>y_{0}
$$

which is not possible and therefore we must integrate (B.4) in the form,

$$
-\sqrt{\frac{7 b}{H_{0}}} x_{r}=\int_{y_{0}}^{y_{r}} \frac{d y}{\left[\left(H_{0} y_{0}{ }^{1 / 2}-1\right)^{2}-\left(1-H_{0} y^{1 / 2}\right)^{2}\right]^{1 / 2}}
$$

where $y_{r}=y\left(x_{r}\right)$. There is a removable singularity in the integral of (B.5). Set

$$
\left(1-H_{0} y^{1 / 2}\right)=\left(H_{0} y_{0}^{1 / 2}-1\right) \sin \theta=A_{1} \sin \theta
$$

such that

$$
d y=-\frac{2 A_{1}}{H_{0}^{2}}\left(1-A_{1} \sin \theta\right) \cos \theta d \theta
$$

and therefore,

$$
-\frac{2}{H_{0}^{2}} \int_{-\pi / 2}^{\theta_{r}}\left(1-A_{1} \sin \theta\right) d \theta
$$


where $\theta=-\pi / 2$ when $y=y_{0}$ and $\theta=\theta_{r}$ when $y=y_{r}$. Integrating (B.6) gives

$$
x_{r}=\frac{2}{\sqrt{7 b H_{0}^{3}}}\left(\theta_{r}+\pi / 2+A_{1} \cos \theta_{r}\right)
$$

where

$$
\theta_{r}+\pi / 2=\cos ^{-1}\left(\frac{1-H_{0} y_{r}^{1 / 2}}{H_{0} y_{0}^{1 / 2}-1}\right)
$$

and

$$
\cos \theta_{r}=\frac{\left\{\left(H_{0} y_{0}{ }^{1 / 2}-1\right)^{2}-\left(1-H_{0} y_{r}^{1 / 2}\right)^{2}\right\}^{1 / 2}}{A_{1}} .
$$

For $T<T_{r}$

$$
y^{\prime \prime}=\frac{7 b}{2}\left[\frac{y}{y_{r}^{3 / 2}}-H_{0}\right],
$$

which can be solved to give the complementary function,

$$
y_{c f}=A_{2} e^{\lambda x}+B_{2} e^{-\lambda x}
$$

where $A_{2}$ and $B_{2}$ are constants and $\lambda=\sqrt{7 b / 2 y_{r}^{3 / 2}}$, and the particular integral,

$$
y_{p i}=H_{0} y_{r}^{3 / 2}
$$

with the general solution

$$
y=y_{c f}+y_{p i} .
$$

Now $y(0)=y_{r}$ for the lower temperature region which therefore allows us to write

$$
B_{2}=y_{r}\left(1-H_{0} y_{r}{ }^{1 / 2}\right)-A_{2} \text {. }
$$

The temperature gradients for the upper and lower regions must match at $y=y_{r}$. Thus,

$$
2 \lambda A_{2}-\lambda y_{r}\left(1-H_{0} y_{r}{ }^{1 / 2}\right)=-\sqrt{\frac{7 b}{H_{0}}}\left[\left(1-H_{0} y_{0}{ }^{1 / 2}\right)^{2}-\left(1-H_{0} y_{r}{ }^{1 / 2}\right)^{2}\right]^{1 / 2}
$$

which specifies $A_{2}$ and therefore $B_{2}$. The final boundary condition (3.6) allows us to write

$$
y_{e}=A_{2} e^{\lambda x_{e}}+B_{2} e^{-\lambda x_{e}}+H_{0} y_{r}^{3 / 2}
$$

where $y\left(x_{e}\right)=y_{e}$ for the lower region. Thus, if we set

$$
\begin{aligned}
& A_{2}=C_{3}-C_{1} \\
& B_{2}=C_{3}+C_{1}
\end{aligned}
$$


where

$$
\begin{aligned}
C_{1} & =\frac{y_{r}{ }^{3 / 4}}{\sqrt{2 H_{0}}}\left\{\left(1-H_{0} y_{0}{ }^{1 / 2}\right)^{2}-\left(1-H_{0} y_{r}{ }^{1 / 2}\right)^{2}\right\}^{1 / 2}, \\
C_{3} & =\frac{1}{2}\left(y_{r}-H_{0} y_{r}^{3 / 2}\right) \\
\text { and } & \\
C_{2} & =y_{e}-H_{0} y_{r}^{3 / 2},
\end{aligned}
$$

then equation (B.9) can be written as

$$
\left(C_{3}-C_{1}\right) e^{2 \lambda x_{e}}-C_{2} e^{\lambda x_{e}}+\left(C_{3}+C_{1}\right)=0,
$$

which can be solved by the quadratic formula to get,

$$
x_{e}=\sqrt{\frac{2 y_{r}^{3 / 2}}{7 b}} \ln \left(\frac{C_{2}-\sqrt{C_{2}{ }^{2}-4\left(C_{3}^{2}-C_{1}^{2}\right)}}{2\left(C_{3}-C_{1}\right)}\right),
$$

where only the negative root is required. Now the coronal loops we are considering have $x \in[0,1 / 2]$ and thus

$$
x_{r}+x_{e}=1 / 2
$$

and therefore from equations (B.7) and (B.17) we can finally conclude that,

$$
\begin{aligned}
\frac{7 b}{2}= & {\left[2 \sqrt{\frac{2}{H_{0}^{3}}}\left\{\cos ^{-1}\left(\frac{1-H_{0} y_{r}^{1 / 2}}{H_{0} y_{0}^{1 / 2}-1}\right)+\frac{\sqrt{2 H_{0}}}{y_{r}^{3 / 4}} C_{1}\right\}\right.} \\
& \left.+2 y_{r}^{3 / 4} \ln \left(\frac{C_{2}-\sqrt{C_{2}^{2}-4\left(C_{3}^{2}-C_{1}^{2}\right)}}{2\left(C_{3}-C_{1}\right)}\right)\right]^{2} .
\end{aligned}
$$

(ii) $T_{0}, T_{e}<T_{r}$

This loop has its entire temperature structure in the lower region of the radiative loss curve. Thus equation (B.8) applies along the loop length and has the general solution

$$
y=A_{2} e^{\lambda x}+B_{2} e^{-\lambda x}+H_{0} y_{r}^{3 / 2} .
$$

Applying boundary condition (3.5) gives $A_{2}=B_{2}$ and thus (B.19) can be written as

$$
y=2 A_{2} \cosh (\lambda x)+H_{0} y_{r}^{3 / 2} .
$$

We have specified that $y(0)=y_{0}$ which gives

$$
A_{2}=\frac{1}{2}\left(y_{0}-H_{0} y_{r}^{3 / 2}\right)
$$


and since $y(1 / 2)=y_{e}$, then

$$
\lambda=2 \cosh ^{-1}\left(\frac{y_{e}-H_{0} y_{r}^{3 / 2}}{y_{0}-H_{0} y_{r}^{3 / 2}}\right),
$$

or rather

$$
\frac{7 b}{2}=4 y_{r}^{3 / 2}\left[\cosh ^{-1}\left(\frac{y_{e}-H_{0} y_{r}^{3 / 2}}{y_{0}-H_{0} y_{r}^{3 / 2}}\right)\right]^{2} .
$$

These different solutions for $T_{0}(b)$ are shown in Figure 3.7 for $T_{e}=0.01$ and $T_{r}=$ 0.011 in units of $10^{6} \mathrm{~K}$ with different values of $H_{0}$. 


\section{Appendix C}

\section{Derivation of $T(x, t)$ for}

\section{Time-Dependent Heating with}

$\omega \gg 1$

The equations to be investigated are

$$
\begin{gathered}
\frac{\partial \rho}{\partial t}+\frac{\partial(\rho v)}{\partial x}=0 \\
\frac{\partial v}{\partial x}=\frac{\partial}{\partial x}\left(T^{5 / 2} \frac{\partial T}{\partial x}\right)-T^{-7 / 4}+1+\sin \omega t \\
\rho=\frac{1}{T} .
\end{gathered}
$$

Rewrite (C.1) as

and linearise about $T=1$ using

$$
\frac{\partial T}{\partial t}+v \frac{\partial T}{\partial x}-T \frac{\partial v}{\partial x}=0
$$

$$
\begin{aligned}
T(x, t) & =1+\frac{1}{\omega} T_{1}(x, t) \\
v(x, t) & =v_{1}(x, t)
\end{aligned}
$$

assuming that $\omega \gg 1$. Thus (C.2) and (C.4) become,

$$
\begin{aligned}
\frac{\partial v_{1}}{\partial x} & \approx \frac{1}{\omega} \frac{\partial^{2} T_{1}}{\partial x^{2}}+\frac{7 T_{1}}{4 \omega}+\sin \omega t, \\
\frac{1}{\omega} \frac{\partial T_{1}}{\partial t} & \approx \frac{\partial v_{1}}{\partial x} .
\end{aligned}
$$

Combining (C.7) and (C.8) gives

$$
\frac{1}{\omega} \frac{\partial T_{1}}{\partial t} \approx \frac{1}{\omega} \frac{\partial^{2} T_{1}}{\partial x^{2}}+\frac{7 T_{1}}{4 \omega}+\sin \omega t
$$


which has the boundary conditions

$$
\begin{aligned}
\frac{\partial T_{1}}{\partial x}=0 & \text { at } & x=0 \\
T_{1}=0 & \text { at } & x=1 / 2,
\end{aligned}
$$

and the initial condition,

$$
T_{1}(x)=0 \text {. }
$$

The full solution to (C.9) will be of the form,

$$
T_{1}(x, t)=T_{s}(x, t)+T_{c f}(x, t)
$$

where

$$
T_{s}(x, t)=A(x) \cos \omega t+B(x) \sin \omega t
$$

and

$$
\frac{\partial T_{c f}}{\partial t}=\frac{\partial^{2} T_{c f}}{\partial x^{2}}+\frac{7 T_{c f}}{4} .
$$

Firstly consider the boundary layer solution to $T_{s}(x, t)$. Substituting (C.14) into (C.9) gives

$$
-A \sin \omega t+B \cos \omega t=\frac{1}{\omega}\left(A^{\prime \prime} \cos \omega t+B^{\prime \prime} \sin \omega t\right)+\sin \omega t,
$$

where $A^{\prime \prime}\left(B^{\prime \prime}\right)$ is the second derivative of $A(B)$ with respect to $x$. Collecting cosine terms gives

$$
B=\frac{A^{\prime \prime}}{\omega}
$$

while for the sine terms,

$$
-A=\frac{B^{\prime \prime}}{\omega}+1
$$

The boundary conditions (C.10) and (C.11) give

$$
\begin{array}{ll}
A(1 / 2)=0, & A^{\prime}(0)=0 \\
B(1 / 2)=0, & B^{\prime}(0)=0,
\end{array}
$$

and therefore $A^{\prime \prime}(1 / 2)=0$ from (C.17). Differentiating (C.17) twice and substituting into (C.18) gives

$$
\frac{A^{i v}}{\omega^{2}}+A+1=0
$$

where $A^{i v}$ is the fourth derivative of $A$ and for which a general solution could be

$$
A=-1+a \mathrm{e}^{\lambda(x-1 / 2)}
$$


where $a$ is a general constant. This gives,

$$
\frac{\lambda}{\omega^{1 / 2}}=(-1)^{1 / 4}=\mathrm{e}^{i(2 n+1) \pi / 4} \quad \text { for } \mathrm{n}=0,1,2, \cdots,
$$

that is

$$
\lambda=\left(\frac{\omega}{2}\right)^{1 / 2}( \pm 1 \pm i)
$$

in all combinations for the four roots available. Thus, equation(C.22) now reads as

$$
A=-1+a_{1} \mathrm{e}^{X_{1}(1+i)}+a_{2} \mathrm{e}^{X_{1}(1-i)}+a_{3} \mathrm{e}^{X_{1}(-1+i)}+a_{1} \mathrm{e}^{X_{1}(-1-i)},
$$

where $a_{1}-a_{4}$ are constants and

$$
X_{1}(x)=\left(\frac{\omega}{2}\right)^{1 / 2}(x-1 / 2) .
$$

Since $\dot{x} \in[0,1 / 2]$ then $X_{1} \leq 0$ for all $x$ and since we will only require the exponential modes to decay, we will ignore $a_{3}$ and $a_{4}$. Applying the boundary condition $A^{\prime \prime}(1 / 2)=0$ gives

$$
a_{1}(1+i)^{2}+a_{2}(1-i)^{2}=0
$$

and therefore,

$$
a_{1}=-a_{2} \mathrm{e}^{-i \pi}=a_{2}
$$

and since $A(1 / 2)=0$ we get

$$
a_{1}=a_{2}=1 / 2 \text {. }
$$

Thus (C.25) can be written as,

$$
A=-1+\frac{1}{2} \mathrm{e}^{X_{1}(1+i)}+\frac{1}{2} \mathrm{e}^{X_{1}(1-i)},
$$

or rather,

$$
A(x)=-1+\mathrm{e}^{X_{1}} \cos X_{1} .
$$

Now from equation (C.17),

$$
B(x)=\frac{A^{\prime \prime}}{\omega}=\frac{1}{2} \frac{d^{2} A}{d X_{1}{ }^{2}},
$$

and thus,

$$
B(x)=-\mathrm{e}^{X_{1}} \sin X_{1},
$$

noting that $B(1 / 2)=0$ as required. Thus the solution to (C.14) is

$$
T_{s}(x, t)=-\cos \omega t+\mathrm{e}^{X_{1}} \cos X_{1} \cos \omega t-\mathrm{e}^{X_{1}} \sin X_{1} \sin \omega t,
$$

which can be rewritten as,

$$
T_{s}(x, t)=-\cos \omega t+\mathrm{e}^{X_{1}} \cos \left(X_{1}+\omega t\right) .
$$


Note that this is only the contribution from the boundary layer at one side of the system $(x=1 / 2)$. There will be a second boundary layer at $x=-1 / 2$ and therefore by a similar analysis, the solution (C.35) will then become,

$$
T_{s}(x, t)=-\cos \omega t+\mathrm{e}^{X_{1}} \cos \left(X_{1}+\omega t\right)+\mathrm{e}^{-X_{2}} \cos \left(X_{2}-\omega t\right)
$$

where

$$
X_{2}(x)=\left(\frac{\omega}{2}\right)^{1 / 2}(x+1 / 2)
$$

and where it must be noted that each boundary layer has been treated independently.

Next, the complementary function $T_{c f}$ can be solved. It satisfies,

$$
\frac{\partial T_{c f}}{\partial t}=\frac{\partial^{2} T_{c f}}{\partial x^{2}}+\frac{7 T_{c f}}{4} .
$$

which has the boundary conditions,

$$
\begin{array}{ll}
\frac{\partial T_{c f}}{\partial x}=0 & \text { at } x=0 \\
T_{c f}=0 & \text { at } x=1 / 2,
\end{array}
$$

and an initial condition which comes from equations (C.13) and (C.36);

$$
T_{c f}(x)=-T_{s}(x) \quad \text { at } \mathrm{t}=0 .
$$

Equation (C.38) can be solved by separation of variables to give,

$$
T_{c f}(x, t)=\mathrm{e}^{-\nu^{2} t}(C \cos \varphi x+D \sin \varphi x),
$$

where $C$ and $D$ are constants and

$$
\varphi=\sqrt{\nu^{2}+7 / 4}
$$

Boundary condition (C.39) gives

$$
\varphi \mathrm{e}^{-\nu^{2} t} D=0
$$

and therefore $D=0$ and so

$$
T_{c f}(x)=C \mathrm{e}^{-\nu^{2} t} \cos \varphi x
$$

and from (C.40)

$$
C \mathrm{e}^{-\nu^{2} t} \cos \frac{\varphi}{2}=0
$$

and thus it follows that

$$
\frac{\varphi}{2}=(n+1 / 2) \pi, \quad n=0,1,2, \cdots
$$


and therefore,

$$
\nu^{2}=(2 n+1)^{2} \pi^{2}-7 / 4
$$

Thus, the series solution is

$$
T_{c f}(x, t)=\sum_{n=0}^{\infty} C_{n} \cos [(2 n+1) \pi x] \mathrm{e}^{-\left[(2 n+1)^{2} \pi^{2}-7 / 4\right] t},
$$

where the $C_{n}$ constants can be specified from the initial condition (C.41); that is,

$$
\sum_{n=0}^{\infty} C_{n} \cos (2 n+1) \pi x=1-\mathrm{e}^{X_{1}} \cos X_{1}-\mathrm{e}^{-X_{2}} \cos X_{2} .
$$

Multiplying each side of (C.49) by $\cos (2 m+1) \pi x$ (where $m$ is an integer) and integrating along the loop gives

$$
\frac{1}{4} C_{m}=\int_{0}^{1 / 2}-T_{s}(x) \cos (2 m+1) \pi x d x,
$$

as $n=m$. If we assume that the contribution from the exponential terms is small, then the solution to (C.50) can be approximated by

$$
C_{m}=4 \int_{0}^{1 / 2} \cos (2 m+1) \pi x d x
$$

and thus

$$
C_{m}=\frac{(-1)^{m}}{(2 m+1) \pi}
$$

Thus the final solution is

$$
\begin{aligned}
T(x, t)=1 & +\frac{1}{\omega}\left[-\cos \omega t+\mathrm{e}^{X_{1}} \cos \left(X_{1}+\omega t\right)+\mathrm{e}^{-X_{2}} \cos \left(X_{2}-\omega t\right)\right] \\
& +\frac{1}{\omega} \sum_{n=0}^{\infty} \frac{(-1)^{n}}{(2 n+1) \pi} \cos [(2 n+1) \pi x] \mathrm{e}^{-\left[(2 n+1)^{2} \pi^{2}-7 / 4\right] t} .
\end{aligned}
$$

If we are considering the evolution of the summit temperature only then (C.53) reduces to

$$
\begin{aligned}
T(0, t)=1 & +\frac{1}{\omega}\left[-\cos \omega t+2 \mathrm{e}^{-\frac{1}{2}(\omega / 2)^{1 / 2}} \cos \left(-\frac{1}{2}\left(\frac{\omega}{2}\right)^{1 / 2}+\omega t\right)\right] \\
& +\frac{1}{\omega} \sum_{n=0}^{\infty} \frac{(-1)^{n}}{(2 n+1) \pi} \mathrm{e}^{-\left[(2 n+1)^{2} \pi^{2}-7 / 4\right] t} .
\end{aligned}
$$




\section{Appendix D}

\section{Analytical Calculation of Solutions to the Dispersion Relation}

A general solution to the dispersion relation

$$
\sigma^{3}+\left\{\epsilon_{0}\left(k^{2}-b\right)+k^{2} \eta\right\} \sigma^{2}+k^{2}\left\{\eta \epsilon_{0}\left(k^{2}-b\right)+\gamma\right\} \sigma+k^{2} \epsilon_{0}\left(k^{2}-b\right)=0
$$

that depends on $\epsilon_{0}, b, \eta$ and $\gamma$, can be found using the mathematical package MAPLE. The three roots $\left(\sigma_{1,2,3}\right)$ are

$$
\begin{aligned}
\sigma_{1} & =A_{1}^{1 / 3}-A_{2}-\frac{1}{3}\left(\eta k^{2}+\epsilon_{0}\left(k^{2}-b\right)\right), \\
\sigma_{2,3} & =-\frac{1}{2}\left(A_{1}^{1 / 3}-A_{2}\right)-\frac{1}{3}\left(\eta k^{2}+\epsilon_{0}\left(k^{2}-b\right)\right) \pm \frac{1}{2} \mathrm{i} \sqrt{3}\left(\mathrm{~A}_{1}^{1 / 3}+\mathrm{A}_{2}\right),
\end{aligned}
$$

where

$$
\begin{array}{r}
A_{1}=\frac{1}{6} k^{4} \gamma \epsilon_{0}-\frac{1}{6} k^{2} \gamma \epsilon_{0} b+\frac{1}{6} k^{4} \gamma \eta+\frac{1}{18} k^{6} \eta \epsilon_{0}^{2}-\frac{1}{9} k^{4} \eta \epsilon_{0}^{2} b+\frac{1}{18} k^{6} \eta^{2} \epsilon_{0} \\
+\frac{1}{18} k^{2} \eta \epsilon_{0}^{2} b^{2}-\frac{1}{18} k^{4} \eta^{2} \epsilon_{0} b-\frac{1}{2} \epsilon_{0} k^{4}+\frac{1}{2} \epsilon_{0} k^{2} b-\frac{1}{27} \epsilon_{0}^{3} k^{6}+\frac{1}{9} \epsilon_{0}^{3} k^{4} b \\
-\frac{1}{9} \epsilon_{0}^{3} k^{2} b^{2}+\frac{1}{27} \epsilon_{0}^{3} b^{3}-\frac{1}{27} \eta^{3} k^{6} \\
+\frac{\sqrt{3} k}{18}\left(4 k^{4} \gamma^{3}+27 \epsilon_{0}^{2} k^{6}+4 \epsilon_{0}^{4} k^{8}+8 k^{8} \gamma \eta^{2} \epsilon_{0}^{2}-2 k^{8} \gamma \eta \epsilon_{0}^{3}\right. \\
-k^{6} \gamma^{2} \epsilon_{0}^{2}-k^{6} \gamma^{2} \eta^{2}+2 k^{10} \eta^{3} \epsilon_{0}^{3}-k^{10} \eta^{2} \epsilon_{0}^{4}-k^{10} \eta^{4} \epsilon_{0}^{2}
\end{array}
$$




$$
\begin{array}{r}
-18 k^{6} \gamma \epsilon_{0}^{2}-6 k^{8} \eta \epsilon_{0}^{3}-6 k^{8} \eta^{2} \epsilon_{0}^{2}-54 \epsilon_{0}^{2} k^{4} b-16 \epsilon_{0}^{4} k^{6} b \\
+24 \epsilon_{0}^{4} k^{4} b^{2}-16 \epsilon_{0}^{4} k^{2} b^{3}+4 \epsilon_{0} k^{8} \eta^{3}+27 \epsilon_{0}^{2} k^{2} b^{2}+36 k^{4} \gamma \epsilon_{0}^{2} b \\
-2 k^{8} \gamma \eta^{3} \epsilon_{0}+10 k^{6} \gamma^{2} \eta \epsilon_{0}-10 k^{4} \gamma^{2} \eta \epsilon_{0} b+2 k^{4} \gamma^{2} \epsilon_{0}^{2} b-k^{2} \gamma^{2} \epsilon_{0}^{2} b^{2} \\
-16 k^{6} \gamma \eta^{2} \epsilon_{0}^{2} b+6 k^{6} \gamma \eta \epsilon_{0}^{3} b-6 k^{4} \gamma \eta \epsilon_{0}^{3} b^{2}+8 k^{4} \gamma \eta^{2} \epsilon_{0}^{2} b^{2} \\
+2 k^{2} \gamma \eta \epsilon_{0}^{3} b^{3}+2 k^{6} \gamma \eta^{3} \epsilon_{0} b-6 k^{8} \eta^{3} \epsilon_{0}^{3} b+4 k^{8} \eta^{2} \epsilon_{0}^{4} b \\
-6 k^{6} \eta^{2} \epsilon_{0}^{4} b^{2}+6 k^{6} \eta^{3} \epsilon_{0}^{3} b^{2}+4 k^{4} \eta^{2} \epsilon_{0}^{4} b^{3}+2 k^{8} \eta^{4} \epsilon_{0}^{2} b \\
-2 k^{4} \eta^{3} \epsilon_{0}^{3} b^{3}-k^{2} \eta^{2} \epsilon_{0}^{4} b^{4}-k^{6} \eta^{4} \epsilon_{0}^{2} b^{2}-18 k^{2} \gamma \epsilon_{0}^{2} b^{2} \\
-18 k^{6} \gamma \eta \epsilon_{0}+18 k^{4} \gamma \eta \epsilon_{0} b+18 k^{6} \eta \epsilon_{0}^{3} b-18 k^{4} \eta \epsilon_{0}^{3} b^{2} \\
\left.+12 k^{6} \eta^{2} \epsilon_{0}^{2} b+6 k^{2} \eta \epsilon_{0}^{3} b^{3}-6 k^{4} \eta^{2} \epsilon_{0}^{2} b^{2}+4 \epsilon_{0}^{4} b^{4}-4 \epsilon_{0} k^{6} b \eta^{3}\right)^{1 / 2}
\end{array}
$$

and

$$
A_{2}=\frac{\left(3 k^{2} \gamma+k^{4} \eta \epsilon_{0}-k^{2} \eta \epsilon_{0} b-\epsilon_{0}^{2} k^{4}+2 \epsilon_{0}^{2} k^{2} b-\epsilon_{0}^{2} b^{2}-\eta^{2} k^{4}\right)}{9 A_{1}} .
$$




\section{Appendix E}

\section{Asymptotic Expansion of the Dispersion Relation}

From equation (6.39), assume that $\epsilon_{0}=b=1$ such that the dispersion relation reads as

$$
\sigma^{3}+\left(k^{2}-1\right) \sigma^{2}+2 k^{2} \sigma+k^{2}\left(k^{2}-1\right)=0 .
$$

Firstly, set

$$
\sigma=a_{0} k^{2}+a_{1}+\cdots
$$

where $a_{0}$ and $a_{1}$ are constant coefficients. Substituting this expansion into equation (E.1) and gathering all the terms in $k^{6}$ gives,

$$
a_{0}^{3}+a_{0}^{2}=0
$$

and therefore $a_{0}=-1$ ( $a_{0}=0$ can be ignored). Collecting all the terms in $k^{4}$ gives,

$$
a_{0}^{2}\left(3 a_{1}-1\right)+2 a_{0} a_{1}+1=0
$$

and therefore, $a_{1}=2$. Thus, we have calculated one root,

$$
\sigma \approx-k^{2}+2
$$

which corresponds to the radiative mode $\left(\sigma_{1}\right)$. Next set,

$$
\sigma=a_{0} k+a_{1}+\frac{1}{k} a_{2}+\frac{1}{k^{2}} a_{3} \cdots
$$

where $a_{0}-a_{3}$ are constant coefficients. Substituting into (E.1) gives

$$
a_{0}= \pm \mathrm{i}, \quad \mathrm{a}_{1}=-\frac{1}{2}, \quad \mathrm{a}_{2}= \pm \frac{3 \mathrm{i}}{8}, \quad \mathrm{a}_{3}=-\frac{1}{2},
$$


each term being calculated from gathering together decreasing powers of $k$ from $k^{4}$. Thus, the second and third roots $\left(\sigma_{2,3}\right)$ of (E.1) are estimated as

$$
\sigma \approx-\frac{1}{2}\left(1+\frac{1}{k^{2}}\right) \pm \mathrm{i} k\left(1+\frac{3}{8 k^{2}}\right)
$$

which corresponds to the thermally damped sound waves. 


\section{Appendix F}

\section{Numerical Algorithm for Reduced Isobaric Equations}

The system of equations to be solved numerically is

$$
\begin{aligned}
\frac{\partial \rho}{\partial t}+\frac{\partial(\rho v)}{\partial x} & =0 \\
\frac{\partial v}{\partial x} & =\frac{\partial}{\partial x}\left(T^{5 / 2} \frac{\partial T}{\partial x}\right)-b\left[\chi T^{\alpha-2}-H\right], \\
\rho & =\frac{1}{T},
\end{aligned}
$$

as derived in Section 2.5 with boundary conditions (2.41), (2.42) and

$$
H(t)=H_{0}(1+\sin \omega t)
$$

The process is started from a hot, static solution obtained by setting $\omega=0$. The basic scheme is to use the temperature profile to estimate the velocity by integrating equation (F.2). The mass continuity equation (F.1) is then used to compute the density at the next timestep from which updated temperature values are generated.

Using a finite-difference grid of size $h$ with a timestep of size $k$, and introducing the notation,

$$
\begin{aligned}
\rho_{q}^{s} & \simeq \rho(q h, s k), \\
T_{q}^{s} & \simeq T(q h, s k), \\
v_{q+1 / 2}^{s} & \simeq v\left(\left(q+\frac{1}{2}\right) h, s k\right),
\end{aligned}
$$

then (F.1) can be approximated by

$$
\rho_{q}^{s+1}=\rho_{q}^{s}-\frac{k}{h} \delta_{x}(\rho v)_{q}^{s}+\frac{k^{2}}{2 h^{2}} \delta_{x}\left(v_{q}^{s} \delta_{x}(\rho v)_{q}^{s}\right)
$$


where $\delta_{x}$ is the central difference operator in the spatial direction and

$$
\rho_{q \pm 1 / 2}^{s}=\frac{\rho_{q \pm 1}^{s}+\rho_{q}^{s}}{2} .
$$

The velocities at the mid-grid values required in (F.5) are obtained recursively from

$$
v_{q+1 / 2}^{s}=v_{q-1 / 2}^{s}+h f_{q}^{s}, \quad q \geq 1,
$$

where

$$
f_{q}^{s}=\frac{1}{h^{2}} \delta_{x}\left[\left(T_{q}^{s}\right)^{5 / 2} \delta_{x}\left(T_{q}^{s}\right)\right]-b\left[\chi\left(T_{q}^{s}\right)^{\alpha-2}-H(t)\right]
$$

and $v_{1 / 2}^{s}=h f_{0}^{s} / 2$. The velocity at the grid points is calculated from

$$
v_{q+1}^{s}=v_{q}^{s}+h f_{q+1 / 2}^{s}, \quad q \geq 1,
$$

where

$$
f_{q \pm 1 / 2}^{s}=\frac{f_{q \pm 1}^{s}+f_{q}^{s}}{2},
$$

and $v_{0}^{s}=0$. New temperature values are then obtained directly from

$$
T_{q}^{s+1}=\frac{1}{\rho_{q}^{s+1}} .
$$

If $2 T^{7 / 2}>|v| h$, conduction is the dominant process and then the usual constraint applies:

$$
\frac{k}{h^{2}} \leq \frac{1}{2 T^{7 / 2}} .
$$

Otherwise, a simplified, linearised stability analysis suggests that an appropriate constraint is given by

$$
k \leq \min _{q}\left\{\frac{-T^{7 / 2}+\sqrt{T^{7}+v^{2} h^{2}}}{v^{2}}\right\} .
$$

In practice, smaller values of $k$ were taken to make allowance for the strongly nonlinear nature of the problem. One advantage in using central differences is that, the approximations not only are second order accurate, but the formulae are symmetric which overcomes the need to monitor the direction of flow especially in a system where the temperature distribution pulses to and fro about its equilibrium state. 


\section{Appendix G}

\section{Numerical Algorithm for Inertial Equations}

The system of equations to be solved numerically is

$$
\begin{aligned}
\frac{\partial \rho}{\partial t}+\frac{\partial(\rho v)}{\partial x} & =0 \\
\frac{\partial(\rho v)}{\partial t}+\frac{\partial\left(\rho v^{2}\right)}{\partial x} & =-\frac{\partial p}{\partial x}+\eta \frac{\partial^{2} v}{\partial x^{2}} \\
\frac{\partial T}{\partial t}+\frac{\partial(v T)}{\partial x} & =\frac{\epsilon_{0}}{\rho}\left[\frac{\partial}{\partial x}\left(T^{5 / 2} \frac{\partial T}{\partial x}\right)-b\left(p^{2} \chi T^{\alpha-2}+H\right)\right] \\
& -(\gamma-2) T \frac{\partial v}{\partial x} \\
p & =\rho T
\end{aligned}
$$

as derived in Section 6.1 with boundary conditions (2.41), (2.42) and

$$
H(t)=H_{0}(1+\sin \omega t) .
$$

The process is started from a hot steady solution obtained by setting $\omega=0$. As before, let $h$ and $k$ denote the $x$ and $t$ grid-spacing respectively. Using the same notation as in Appendix F, a MacCormack Conservative Law Difference Method (DuChateau and Zachmann, 1989) is applied to equation (G.1);

$$
\begin{aligned}
\rho_{q}^{*} & =\rho_{q}^{s}-\frac{k}{h}\left((\rho v)_{q}^{s}-(\rho v)_{q-1}^{s}\right), \\
\rho_{q}^{s+1} & =\frac{1}{2}\left[\rho_{q}^{s}+\rho_{q}^{*}-\frac{k}{h}\left(\rho_{q+1}^{*} v_{q+1}^{s}-\rho_{q}^{*} v_{q}^{s}\right)\right] .
\end{aligned}
$$


Similarly for equation (G.2);

$$
(\rho v)_{q}^{*}=(\rho v)_{q}^{s}-\frac{k}{h}\left(v_{q}^{s}(\rho v)_{q}^{s}-v_{q-1}^{s}(\rho v)_{q-1}^{s}\right)
$$

with

then,

$$
(\rho v)_{n}^{*}=\frac{1}{3}\left(4(\rho v)_{n-1}^{*}-(\rho v)_{n-2}^{*}\right), \quad n h=1 / 2 ;
$$

$$
\begin{aligned}
(\rho v)_{q}^{s+1} & =\frac{1}{2}\left[(\rho v)_{q}^{s}+(\rho v)_{q}^{*} \frac{k}{h}\left(v_{q+1}^{s}(\rho v)_{q+1}^{*}-v_{q}^{s}(\rho v)_{q}^{*}\right)\right] \\
& -\frac{k}{2 h}\left(p_{q+1}^{s}-p_{q-1}^{s}\right) \\
& +\frac{k \eta}{h^{2}}\left(v_{q+1}^{s}+v_{q-1}^{s}-2 v_{q}^{s}\right),
\end{aligned}
$$

with

$$
(\rho v)_{n}^{s+1}=\frac{1}{3}\left(4(\rho v)_{n-1}^{s+1}-(\rho v)_{n-2}^{s+1}\right), \quad n h=1 / 2 .
$$

New velocity values are obtained from

$$
v_{q}^{s+1}=\frac{(\rho v)_{q}^{s+1}}{\rho_{q}^{s+1}} .
$$

The energy equation (G.3) is differenced as

$$
\begin{aligned}
T_{q}^{s+1} & =T_{q}^{s}+\frac{k \epsilon_{0}}{\rho_{q}^{s}}\left(\frac{1}{h^{2}} \delta_{x}\left[\left(T_{q}^{s}\right)^{5 / 2} \delta_{x}\left(T_{q}^{s}\right)\right]-b\left[\left(p_{q}^{s}\right)^{2} \chi\left(T_{q}^{s}\right)^{\alpha-2}-H(t)\right]\right) \\
& -\frac{k}{2 h}\left(v_{q+1}^{s} T_{q+1}^{s}-v_{q-1}^{s} T_{q-1}^{s}\right) \\
& -\frac{k}{2 h} T_{q}^{s}\left(v_{q+1}^{s}-v_{q-1}^{s}\right) .
\end{aligned}
$$

The new pressure values are then calculated from

$$
p_{q}^{s+1}=\rho_{q}^{s+1} T_{q}^{s+1} .
$$

Various timestep constraints from the conduction,

$$
\frac{k}{h^{2}} \leq \min _{q}\left\{\frac{\rho}{\epsilon_{0} T^{5 / 2}}\right\}
$$

the CFL condition,

$$
\frac{k}{h} \leq \min _{q}\left\{\frac{1}{v}\right\}
$$


and the acoustic term,

$$
\frac{k}{h} \leq \min _{q}\left\{\frac{\rho v}{p}\right\}
$$

were calculated and the value of $k$ used was taken to be much smaller than the minimum of the above to make allowance for the nonlinear aspects of the problem. 


\section{Bibliography}

[1] Athay, R.G., 1966, "Theoretical Line Intensities V: Solar UV Emission Lines of Heavy Elements", Astrophys. J., 145, 784.

[2] Athay, R.G., 1984, "The Origin of Spicules and Heating of the Lower Transition Region", Astrophys. J., 287, 412.

[3] Airapetian, V.S. and Smartt, R.N., 1994, "Role of Loop-Loop Encounters in Coronal Heating", Proc. of Third SOHO Workshop, ESASP-373, p29.

[4] Airapetian, V.S. and Smartt, R.N., 1995, "Optical Diagnostics of Coronal Loop Interactions", Astrophys. J., 445, 489.

[5] Alissandrakis, C.E., Schadee, A. and Kundu, M.R., 1988, "High Resolution Microwave and X-ray Observations of Solar Flares", Astron. \& Astrophys., 195,290 .

[6] Anderson, L.S. and Athay, R.G., 1989a, "Chromospheric and Coronal Heating", Astrophys. J., 336, 1089.

[7] Anderson, L.S. and Athay, R.G., 1989b, "Model Solar Chromosphere with Prescribed Heating", Astrophys. J., 346, 1010.

[8] Antiochos, S.K., 1979, "The Stability of Solar Coronal Loops", Astrophys. J., 232, L125.

[9] Antiochos, S.K. and Noci, G., 1986, "The Structure of the Static Corona and Transition Region", Astrophys. J., 301, 440.

[10] Bender, C.M. and Orzag, S.A., 1978, Advanced Mathematical Methods for Scientists and Engineers, $\mathrm{M}^{\mathrm{c}} \mathrm{Gram}-\mathrm{Hill}$, New York.

[11] Braginsky, S.I., 1965, Rev. Plasma Phys., 1, 205.

[12] Brown, S.F., 1995, "Formation of Coronal Loops with Temperatures Between $10^{5} \mathrm{~K}$ and $10^{6} \mathrm{~K}$ ", Astron. \& Astrophys., submitted. 
[13] Butler, C.J., Rodono, M., Foing, B.H. and Haisch, B.M., 1986, Nature, 312 , 679.

[14] Cally, P.S. and Robb, T.D., 1995, "Stability, Structure and Evolution of Cool Loops", Astron. \& Astrophys., submitted.

[15] Cargill, P.J., 1993, "The Fine Structure of a Nanoflare Heated Corona", Solar Phys., 147, 263.

[16] Cargill, P.J., 1994a, "The Experimental Signatures of Coronal Heating Mechanisms", Solar System Plasmas in Space and Time, Geophysical Monograph 84 .

[17] Cargill, P.J., 1994b, "Some Implications of the Nanoflare Concept", Astrophys. $J ., \mathbf{4 2 2}, 381$.

[18] Cargill, P.J., 1995, private communication.

[19] Cook, J.W., Cheng, C.-C., Jacobs, V.L. and Antiochos, S.K., 1989, "Effect of Coronal Elemental Abundances of the Radiative Loss Function", Astrophys. $J ., 338,1176$.

[20] Craig, I.J.D., Robb, T.D. and Rollo, M.D., 1982, "The Stability and Uniqueness of Coronal Loops", Solar Phys., 76, 331.

[21] Davila, J.M., 1987, "Heating of the Solar Corona by Resonant Absorption of Alfvén Waves", Astrophys. J.,317, 514.

[22] Dere, K.P. and Mason, H.E., 1993, "Nonthermal Velocities in the Solar Transition Zone Observed with HRTS", Solar Phys., 144, 217.

[23] Domingo, V., Fleck, B. and Poland, A. I., 1994, "The Scientific Payload of the Space-Based Solar and Heliospheric Observatory (SOHO)", Space Science Reviews, 70, 7.

[24] Doschek, G.A., 1995, "Observations of Nanoflares with FeXVII, FeXVIII and FeXIX Lines", SUMER Scientific Report, Version 3.2, p8-56.

[25] Doyle, J.G., 1995, "Chromospheric Oscillations", CDS Scientific Report, Version $6, \mathrm{p} 14-26$.

[26] Doyle, J.G., 1995, "Chromospheric Oscillations", SUMER Scientific Report, Version 3.2, p8-77. 
[27] DuChateau, P. and Zachmann, D., 1989, Applied Partial Differential Equations, Harper \& Row, 450.

[28] Erdelyi, R. and Goossens, M., 1995, "Resonant Absorption of Alfvén Waves in Coronal Loops in Visco-resistive MHD", Astron. \& Astrophys., 294, 575.

[29] Fiedler, R.A. and Hood, A.W., 1993, "The Effect of Shear on Numerical Models of Quiescent Normal Polarity Promeniences", Solar Phys., 146, 297.

[30] Forbes, T.G. and Priest, E.R., 1982, "Numerical Study of Line-tied Magnetic Reconnection", Solar Phys., 81, 303.

[31] Foukal, P., 1981, "The $\mathrm{C}_{\mathrm{I}}$ Opacity and Physical Structure of Cool, Very Dense Pḷasma in the Solar Corona", Astrophys. J., 245, 304.

[32] Glasgaard, K. and Nordlund, A., 1995, "The Heating and Activity of the Solar Corona: I. Boundary Shearing of an Initially Homogeneous magnetic Field", J. Geophys. Res., in press.

[33] Gómez, D.O., Martens, P.C.H. and Golub, L., 1993a, "Normal Incidence Xray Telescope Power Spectra of X-ray Emission from Solar Active Regions I: Observations", Astrophys. J., 405, 767.

[34] Gómez, D.O., Martens, P.C.H. and Golub, L., 1993b, "Normal Incidence Xray Telescope Power Spectra of X-ray Emission from Solar Active Regions II: Theory", Astrophys. J., 405, 773.

[35] Hansteen, V. and Judge, P., 1995, "Evidence for Nanoflares in Coronal and Transition Region Lines", CDS Scientific Report, Version 6, p14-65.

[36] Harrison, R.A. and Fludra, A., 1995, CDS Scientific Report, Version 6.

[37] Harrison, R.A. and Gurman, J., 1995, "Microflare Study", CDS Scientific Report, Version 6, p14-59.

[38] Harrison, R.A. and Priest, E.R., 1995, "Elementary Coronal Heating Events", CDS Scientific Report, Version 6, p14-42.

[39] Hassler, D.M., Rottman, G.J., Shoub, E.C. and Holzer, T.E., 1990, "Line Broadening of $\mathrm{MgX} \lambda \lambda 609$ and 625 Coronal Emission Lines Observed Above the Solar Limb", Astrophys. J., 348, L77.

[40] Heinzel, P., 1994, "Dynamic Loops in the Corona", Proc. of Third SOHO Workshop, ESA SP373, pp133-138. 
[41] Heyvaerts, J. and Priest, E.R., 1983, "Coronal Heating by Phase-mixed Shear Alfvén Waves", Astron. Astrophys., 117, 220.

[42] Heyvaerts, J. and Priest, E.R., 1992, "A Self-Consistent Turbulent Model for Solar Coronal Heating", Astrophys. J., 390, 297.

[43] Hildner, E., 1974, "The Formation of Solar Quiescent Prominences by Condensation", Solar Phys., 35, 123.

[44] Hollweg, J.V., 1984, "Resonances in Coronal Loops", Astrophys. J., 277, 392.

[45] Hollweg, J.V., 1986, "Transition Region, Corona and Solar Wind in Coronal Holes", J. Geophys. Res., 91, 4111.

[46] Hood, A.W. and Anzer, U., 1988, "Thermal Condensations in Coronal Magnetic Fields", Solar Phys., 115, 61.

[47] Hood, A.W. and Priest, E.R., 1979, "The Equilibrium of Solar Coronal Loops", Astron. \& Astrophys., 77, 233.

[48] Hood, A.W. and Priest, E.R., 1980, "Are Solar Coronal Loops in Thermal Equilibrium?", Astron. \& Astrophys., 87, 126.

[49] Hudson, H.S., 1991, "Solar Flares, Microflares, Nanoflares and Coronal Heating", Solar Phys., 133, 357.

[50] Hudson, H.S., 1994, "Thermal Plasmas in the Solar Corona: the Yohkoh Soft X-ray Observations", Proc. of Kofu Symposium, NRO Report No.360, p1.

[51] Ionson, J.A., 1978, "Resonant Absorption of Alvénic Surface Waves and Heating of Coronal Loops", Astrophys. J., 226, 650.

[52] Inverarity, G.W., 1995, private communication.

[53] Inverarity, G.W., Priest, E.P. and Heyvaerts, J., 1995, "Turbulent Coronal Heating: I. Shearing Arcade", Astron. \& Astrophys., 293, 913.

[54] Inverarity, G.W. and Priest, E.P. , 1995a, "Turbulent Coronal Heating: II. Twisted Flux Tube", Astron. 63 Astrophys., 296, 395.

[55] Inverarity, G.W. and Priest, E.P. , 1995b, "Turbulent Coronal Heating: III. Wave Heating in Coronal Loops", Astron. \& Astrophys., 302, 567.

[56] Ireland, J., 1995, "Wave Heating in the Solar Corona and SOHO", Annales Geophysicae, in press. 
[57] Klimchuk, J. and Harrison, R.A., 1995, "Filling Factors of Coronal Loops"; CDS Scientific Report, Version 6, p14-40.

[58] Kopp, R.A. and Poletto, G., 1993, "Coronal Heating by Nanoflares: Individual Events and Global Energetics", Astrophys. J., 418, 496.

[59] Lee, M.A. and Roberts, B., 1986, "On the Behaviour of Hydrodynamic Surface Waves", Astrophys. J., 301, 430.

[60] Lin, R.P., Schwartz, A., Kane, S.R., Pelling, R.M. and Hurley, K.C., 1984, "Solar Hard X-ray Microflares", Astrophys. J., 283, 421.

[61] MacNeice, P., 1986, "A Numerical Hydrodynamic Model of a Heated Coronal Loop", Solar Phys., 103, 47.

[62] $\mathrm{M}^{\mathrm{c}} \mathrm{Clements,} \mathrm{K.,} \mathrm{Alexander,} \mathrm{D.} \mathrm{and} \mathrm{Harrison,} \mathrm{R.A.,} \mathrm{1995,} \mathrm{"Observing} \mathrm{Wave}$ Activity with SOHO", CDS Scientific Report, Version 6, p14-109.

[63] M'Whirter, R.W.P., 1995, "Emission Measure Study of Quiet Sun", CDS Scientific Report, Version 6, p14-36.

[64] Mariska, J.T., Boris, J.P., Oran, E.S., Young Jr, T.R. and Doschek, G.A., 1982, "Solar Transition Region Response to Variations in the Heating Rate", Astrophys. J., 225, 783.

[65] Mariska, J.T., 1987, "Observational Signatures of Loop Flows Driven by Asymmetric Heating", Astrophys. J., 334, 489.

[66] Martens, P.C. and Gómez, D.O., 1992, "Spatial Power-Spectra from Yohkoh Soft X-ray Images", PASJ, 44, 187.

[67] Mason, H., 1994, Talk at Advanced PPARC Summer School in Solar Terestrial Physics, QMW College, London.

[68] Mason, H., 1995, "Observations of Nanoflares with FeXXI Emission", SUMER Scientific Report, Version 3.2, p8-39.

[69] Meerson, B., 1989, "The Non-linear Theory of the Thermal Instability: The Intermediate and Short Wavelength Limit", Astrophys. J., 347, 1012.

[70] Morgan, B.J., 1984, Elements of Simulation, Cambridge University Press.

[71] Mok, Y. and Van Hoven, G., 1993, "The Thermal Structure of the Magnetized Solar Transition Region", Solar Phys., 146, 5. 
[72] Moore, R.T., Porter,J., Roumeliotis, G, Tsuneta, S., Shimizu, T, Sturrock. P.A. and Acton, L.W., 1994, "Observations of Enhanced Coronal Heating in Sheared Magnetic Fields", Proc. of Kofu Symposium, NRO Report No360, p89.

[73] Mullan, D.J.R. and Cheng, Q.Q., 1994, "Acoustic Waves in M Dwarfs: Maintaining a Corona", Astrophys. J., 420, 392.

[74] Narain, U. and Ulmschneider, P., 1990, "Chromospheric and Coronal Heating Mechanisms", Space Sci. Rev., 54, 337.

[75] Narain, U. and Ulmschneider, P., 1995, "Chromospheric and Coronal Heating Mechanisms II", Space Sci. Rev., submitted.

[76] Ofman, L. and Davila, J.M., 1994, "Non-linear Studies of Coronal Heating by the Resonant Absorption of Alfvén Waves", Astrophys. J., 247, 686.

[77] Parker, E.N., 1972, "Topological Dissipation and Small Scale Fields in Turbulent Gases", Astrophys. J., 174, 499.

[78] Parker, E.N., 1987a, "Heating of the Stellar Corona", Coronal and Promenience Plasmas, NASA Conference Publication 2442, p9-17.

[79] Parker, E.N., 1987b, "Magnetic Reconnection and the Spontaneous Formation of Tangential Discontinuities in Deformed Magnetic Fields", Astrophys. J., $\mathbf{3 1 8}, 876$.

[80] Parker, E.N., 1988, "Nanoflares and the Solar X-ray Corona", Astrophys. J., 330, 474 .

[81] Parker, E.N., 1991, "The Phase Mixing of Alvén Waves, Coordinated Modes and Coronal Heating", Astrophys. J., 376, 355.

[82] Parker, E.N., 1993, "Resistive Dissipation and Magnetic Field Topology in the Stellar Corona", Astrophys. J., 407, 342.

[83] Peres, G., Rosner, R., Serio, S and Vaiana, G., 1982, "Hydrodynamics and Diagnostics of Coronal Loops Subject to Dynamic Heating", Astrophys. J., 252, 791.

[84] Peres, G., Reale, F. and Serio, S, 1993, "Hydrodynamics and Diagnostics of Coronal Loops Subject to Dynamic Heating", Physics of Solar and Stellar Coronae, eds. J.F.Linksy and S.Serio, pp151-158. 
[85] Poedts, S., Kerner, W. and Goossens, M., 1989, "Alvén Wave Heating In Resistive MHD", J. Plasma Physics, 42.1, 27.

[86] Poedts, S., Goossens, M. and Kerner, W. 1989, "Numerical Simulation of Coronal Heating by Resonant Absorption of Alvén Waves", Solar Phys., 123, 83.

[87] Poedts, S., Belien, J.C. and Goedbloed, J.P., 1994, "On the Quality of Resonant Absorption as a Coronal Loop Heating Mechanism", Solar Phys., 151, 271.

[88] Poletto, G., Kopp, R.A., Hiei, E., Shimizu, T. and Harrison, R.A., 1995, "Coronal Heating Via Nanoflares", CDS Scientific Report, Version 6, p14-60.

[89] Porter, J.G., Toomre, J. and Gebbie K.B., 1984, "Frequent Ultraviolet Brightenings Observed in a Solar Active Region with SMM", Astrophys. J., 283, 879.

[90] Porter, J.G., Moore, R.L. and Reichmann, E.J., 1987, "Microflares in the Solar Magnetic Network", Astrophys. J., 323, 380.

[91] Porter, L.J.., Klimchuk, J.A. and Sturrock, P.A., 1994a, "The Possible Role of MHD Waves in Heating the Solar Corona", Astrophys. J., 435, 482.

[92] Porter, L.J.., Klimchuk, J.A. and Sturrock, P.A., 1994b, "The Possible Role of High Frequency Waves in Heating Solar Coronal Loops", Astrophys. J., 435, 482.

[93] Priest, E.R., 1982, Solar Magnetohydrodynamics, D. Reidel Publ. Co., Dordrecht, Holland.

[94] Priest, E.R., 1993, Physics of Solar and Stellar Coronae, eds. J.F. Linskey and S. Serio, pp $515-532$.

[95] Priest, E.R. and Forbes, T.G., 1989, "Steady Magnetic Reconnection in Three Dimensions", Solar Phys., 119, 211.

[96] Rabin, D. and Moore, R., 1984, "Heating the Sun's Lower Transition Region with Fine Scale Electric Currents", Astrophys. J., 285, 359.

[97] Raymond, J.C. and Doyle, J.G., 1981, "The Energy Balance in Coronal Holes and Average Quiet Sun Regions", Astrophys. J., 247, 686.

[98] Riyopoulos, S., Bondeson, A. and Montgomery, D., 1982, "Relaxation Towards States of Minimum Energy in a Compact Torus", Phys, Fluids, 25(1), 107. 
[99] Rosner, R., Tucker, W.H. and Vaiana, G.S., 1978, "Dynamics of the Quiescent Solar Corona", Astrophys. J., 220, 643.

[100] Rudermann, M.S. and Goossens, M., 1993, "Non-linearity Effects on Resonant Absorption of Surface Alfvén Waves in Incompressible Plasmas", Solar Phys., 143, 69 .

[101] Schmeider, B., Fontela, J., Tandberg-Hanssen, E. and Simnett, G.M., 1994, "Microflares and their Related Events", Proc. of Kofu Symposium, NRO Report No.360, p339.

[102] She, Z.S., Malherbe, J.M. and Raadu, M.A., 1986, "Transition Zone Effects on Thermal Non-Equilibrium and Plasma Condensations in Solar Coronal Loops", Astron. \& Astrophys., 164, 364.

[103] Shimizu, T., Tsuneta, S., Acton, L.W., Lemen, J.R. and Uchida, Y., 1992, "Transient Brightenings in Active Regions Observed by SXT on Yohkoh", PASJ, 44, L147.

[104] Shimizu, T., 1995, "Eneretics and Occurence rate of Active Region Transient Brightenings and Implications for the Heating of the Active Corona", PASJ, 47, 251.

[105] Spitzer, L., 1962, "Physics of Fully Ionized Gases", Interscience, New York.

[106] Sion, E.M., 1995, "The Evolutionary Thermal Response of a White Dwarf to Compressional Heating by Periodic Dwarf Nova Accretion Events", Astrophys. $J ., 438,876$.

[107] Steele, C.D.C., 1992, "Equilibrium and Eruption of Solar Coronal Magnetic Structures", $\mathrm{PhD}$ Thesis.

[108] Steele, C.D.C. and Priest, E.R., 1990a, "Thermal Equilibria of Coronal Magnetic Loops", Solar Phys., 125, 295.

[109] Steele, C.D.C. and Priest, E.R., 1990b, "Thermal Equilibria of Coronal Magnetic Arcades", Solar Phys., 127, 65.

[110] Steele, C.D.C. and Priest, E.R., 1991a, "Thermal Equilibria of Coronal Magnetic Loops with Non-constant Cross-sectional Area", Solar Phys., 132, 293.

[111] Steele, C.D.C. and Priest, E.R., 1991b, "Thermal Equilibria of Isobaric Coronal Magnetic Arcades", Solar Phys., 134, 73. 
[112] Steele, C.D.C. and Priest, E.R., 1994, "Thermal Equilibria in Coronal Magnetic Loops: Gravitational Considerations", Solar Phys., 292, 291.

[113] Strong, K.T., 1994, "Observations of the Structure and Dynamics of Coronal Loops", Proc. of Kofu Symposium, NRO Report No.360, p53.

[114] Sturrock, P.A., Dixon, W.W. and Klimchuk, J.A., 1990, "Episodic Coronal Heating", Astrophys. J., 356, L31.

[115] Van Ballegooijen, A.A., 1986, "Cascade of Magnetic Energy as a Mechanism for Coronal Heating”, Astrophys. J., 311, 1001.

[116] Van Ballegooijen, A.A., 1987, "Cascade Model of Coronal Heating", Coronal and Prominence Plasmas, NASA CP-2442, p439.

[117] Vlahos, L., Georgoulis, M., Kluiving, R. and Paschos, P., 1995, "The Statistical Flare: Nanoflares, Microflares and Flares", Astron. \& Astrophys., 299, 897.

[118] Van Hoven, G. and Mok, Y., 1993, "The Differential Emission Measure of Nested Hot and Cool Magnetic Loops", Solar Phys., 147, 199.

[119] Walsh, R.W., Bell, G.E. and Hood, A.W., 1995a, "Time Dependent Heating of the Solar Corona", Solar Phys., 161, 83.

[120] Walsh, R.W., Bell, G.E. and Hood, A.W., 1995b, "Plasma Flows due to Time Dependent Heating of the Solar Corona", Astrophysical Letters and Communications, eds. K. Tsinganos and A. Ferrari, in press.

[121] Walsh, R.W., Hood, A.W. and Bell, G.E., 1995c, "Modelling ConvectionConduction Subject to Periodic Heating", Recent Research Developments in Heat, Mass and Momentum Transfer, publishers "Research Signpost", submitted.

[122] Wesson, J., 1987, "Tokmaks", The Oxford Engineering Science Series, Volume 20, Oxford University Press.

[123] Wilhelm, K., SUMER Scientific Report, Version 3.2.

[124] Withbroe, G.L. and Noyes, R.W., 1977, "Mass and Energy Flow in the Solar Chromosphere and Corona", Ann. Rev. Astron. Astrophys., 15, 363.

[125] Wragg, M.A. and Priest, E.R., 1981, "The Temperature-Density Structure of Coronal Loops in Hydrostatic Equilibrium", Solar Phys., 70, 293. 
[126] Wright, A.N. and Rickard, G.J., 1995, " A Numerical Study of Resonant Absorption in a Magnetohydrodynamic Cavity Driven by a Broad-band Spectrum", Astrophys. J., 444, 458.

[127] Zirker, J.B., 1993, "Coronal Heating", Solar Phys., 148, 43.

[128] Zirker, J.B. and Cleveland, F.M., 1994, "Searching for Nanoflares", Solar Phys., 153, 245. 